ANL-6515

Instruments

(TID-4500, 17th Ed.)

AEC Research and

Development Report

ARGONNE NATIONAL LABORATORY

9700 South Cass Avenue

Argonne, Illinois

SECOND SYMPOSIUM ON PHYSICS AND NONDESTRUCTIVE TESTING

Held at Argonne National Laboratory

October 3, 4, 5, 1961

Program arranged by:

Nondestructive Testing Group

Metallurgy Division

Program Chairman:

W. J. McGonnagle

Editor:

Roberta Ann di Novi

Operated by The University of Chicago

under

Contract W-31-109-eng-38 


\section{DISCLAIMER}

This report was prepared as an account of work sponsored by an agency of the United States Government. Neither the United States Government nor any agency Thereof, nor any of their employees, makes any warranty, express or implied, or assumes any legal liability or responsibility for the accuracy, completeness, or usefulness of any information, apparatus, product, or process disclosed, or represents that its use would not infringe privately owned rights. Reference herein to any specific commercial product, process, or service by trade name, trademark, manufacturer, or otherwise does not necessarily constitute or imply its endorsement, recommendation, or favoring by the United States Government or any agency thereof. The views and opinions of authors expressed herein do not necessarily state or reflect those of the United States Government or any agency thereof. 


\section{DISCLAIMER}

Portions of this document may be illegible in electronic image products. Images are produced from the best available original document. 
TABLE OF CONTENTS

Page

WELCOMING REMARKS

W. J. McGonnagle.......................... 3

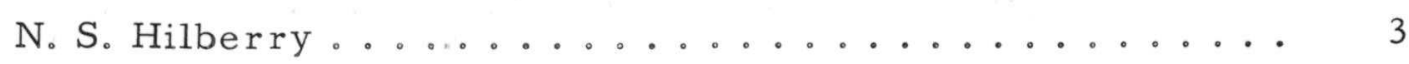

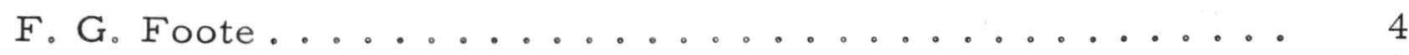

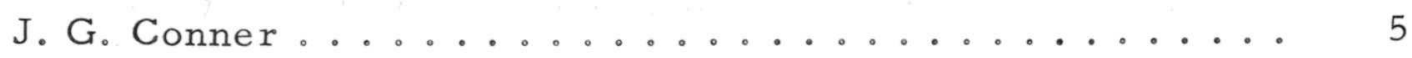

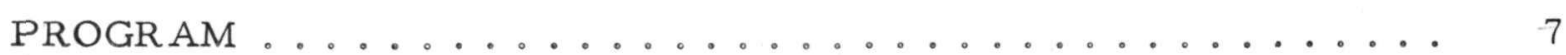

ACTIVATION ANALYSIS FOR MATERIAL TESTING AND RESEARCH

E. A. Burrill - High Voltage Engineering Corporation

J. Hirschfield - High Voltage Engineering Corporation .

NEUTRON RADIOGR APHY: A SECOND PROGRESS

REPORT

H. Berger - Argonne National Laboratory.........

INVESTIGATIONS IN NEUTRON IMAGING

H.V. Watts - Armour Research Foundation .......

THE LATENT IMAGE

A. H. Allard - Eastman Kodak Company .........

MEANING OF THE CHARACTERISTIC CURVES

E. T. Larson - Ansco Corporation

H. F. Nitka - Ansco Corporation............

FACTORS AFFECTING RADIOGRAPHIC SENSITIVITY

W. D. Kiehle - Eastman Kodak Company.........

GENERATION AND DETECTION OF ULTRA HIGH FREQUENCY SOUND WAVES IN SOLIDS

H. E. Bömmel - Bell Telephone Laboratories, Inc....

ULTRASONIC PROPAGATION IN DEFORMED SINGLE AND POLYCRYSTALLINE MATERIALS

F. Rollins - Midwest Research Institute

R. R. Rowand - Wright Air Development Center.....

MECHANICAL VIBRATIONS IN SOLID RODS

R. G. Peterson - Argonne National Laboratory ..... 
TABLE OF CONTENTS

$\underline{\text { Page }}$

SOME OPTICAL AND ELECTRICAL PROPERTIES OF MATERIALS

R. J. Maurer - University of Illinois ........... 162

INFRARED PHYSICS

H. L. Sacks - Perkin-Elmer Corporation ........ 163

THERMISTORS

R. S. Goodyear - Fenwal Electronics, Inc. ....... 187

NONDESTRUCTIVE TESTING OF REACTOR IRRADIATED

METALS

T. H. Blewitt - Argonne National Laboratory. ..... 202

COLOR RADIOGRAPHY

N. S. Beyer - Argonne National Laboratory. ...... 203

SOME DESIGN ASPECTS OF LINEAR ACCELERATORS

USED FOR HIGH ENERGY X-RAY PRODUCTION

J.Haimson - Varian Associates ........... 218

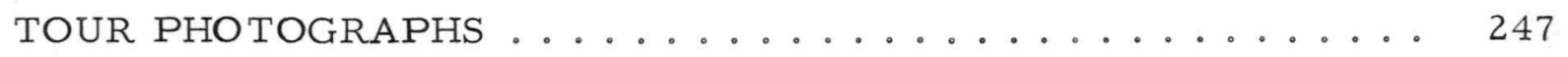

PHOTOGRAPHS AND BIOGRAPHICAL INFORMATION OF

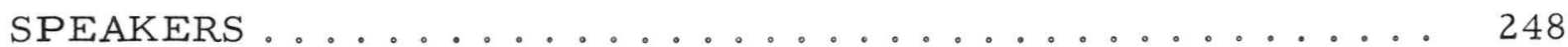

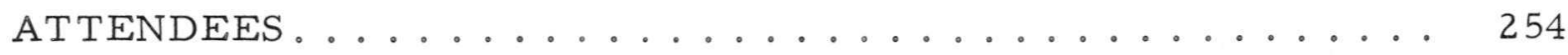




\section{WELCOMING REMARKS}

Warren J. McGonnagle: On behalf of the Argonne National Laboratory, the Metallurgy Division of the Laboratory, and the Nondestructive Testing Group of the Metallurgy Division, I wish to welcome you to another symposium on Physics and Nondestructive Testing. This year we shall once again discuss some of the physical phenomena which can be used to evaluate materials either for wanted or unwanted properties. We never do things by halves here at Argonne, and, so today for our welcoming remarks we have three substitutes. Dr. Hilberry, Director of the Laboratory, is out of town, Dr. Turner will speak for him; Dr. Foote, Director of the Metallurgy Division, is out of town, Mr. Smith will speak for him; and Mr. Conner from the Atomic Energy Commission is on his way out of the country, Mr. Bonnefond will speak for him.

First, I would like to ask Dr. Louis Turner, Deputy Laboratory Director, to say a few words of welcome for the Laboratory. Dr Turner.

Louis A. Turner: Just in order to scotch any possible rumors I think I should say that, so far as I am aware, there is no major scandal to account for so many people being out of town at once. In all serious enterprises these days, as you know, it's important to have strength on the bench. It wouldn't be modest for me to emphasize the strength aspect, but I assure you we've got a lot of people on the bench - so that's where I come in. To judge from the program, this meeting bids fair to be extremely interesting. To be sure, it's the Physics of Nondestructive Testing, but nevertheless, Physics is a pretty broad subject in itself as one can see just by looking at this program. So this meeting, gets more or less, in spite of the limitation implied in the title, into the interdisciplinary field or at least intersubdisciplinary. Now this, as you know, is the great word these days. One must be interdisciplinary and we try our best. In all seriousness, we do claim that this is one of the things that the National Laboratories can be more readily than some other institutions. This is because they are relatively new and they don't have as many traditions; people haven't had the long decades to build up the deep resentments that prevent them from speaking to each other. We do our best to be interdisciplinary in this serious kind of way. And yet, every once in a while we get shocked by the realization that sometimes we know more about what the fellow in Hamburg or Saclay is doing than we do about what the fellow in the other building across this campus is doing. So it's an ideal, but it's one that is taken seriously. We Americans have to get used to recognizing a difference between fine ideals and imperfect attainment of these ideals.

I consider that this meeting is in this spirit and I hope that it will be extremely successful. I feel that this general theme would have been a good idea for a conference for that reason even if it had not had a 
serious and practical aspect to it. But, it certainly does have a serious and practical aspect and the interesting thing about it is that this is the unifying theme, the desire, the necessity of achieving satisfactory nondestructive methods for testing. I'm reminded of a remark which I think was made by the late P.W.Bridgman, namely, that the scientific method, broadly speaking, amounts to very little more than doing your damnedest with no holds barred. Well, I believe that describes the point of view you have to have in this matter of nondestructive testing. And it is serious; it is practical. It can turn extravagant things that show promise into economical practicalities. It can turn the risky and the dangerous into the thoroughly safe.

It gives me great pleasure to welcome all of you to this meeting at Argonne. I hope the meeting will be a success and that all of the auxiliary arrangements will turn out to be satisfactory, and that we ${ }^{8} 11$ all have a fine time. Thank you.

Warren J. McGonnagle: Thank you, Dr。Turner。Our next speaker is Mr. Karl Smith, Assistant Director of the Metallurgy Division.

Karl F.Smith: The interest of our division in nondestructive testing stems from the necessity for having the highest grade of materials possible going into nuclear reactors. If we were dealing with devices, of which the internals were subject to regular servicing, we might be able to emulate the automotive industry. In so doing, we could assemble fuel elements using commercial grades of parts, inspect every tenth component with calipers and magnifying glass, and allow the user a free 1000-mile checkup.

This system is not feasible for a device like a reactor which becomes radioactive with use and which can be serviced only by remotely controlled methods. Instead, we try to make a fuel core as good as we can the first time, seal it closed, and hope all parts last out the lifetime of the fissionable material. This is many times a vain hope, but we are getting better at the business right along, and with it, we must be putting in materials of higher and higher integrity as fuels are made to last longer. As no reactor core is stronger than its weakest link, it behooves us to eliminate such things as voids, stringers, carelessly executed welds, nonadherent claddings, and other such devils of the metallurgical trade. We must do it in every piece - not every tenth piece. By contributing the utmost in integrity to these reactor components, we will increase the life of the reactor core, reduce the frequency of refueling, and bring down the cost of nuclear power.

Your program appears to be a very pertinent and interesting one. On behalf of Argonne Metallurgy Division, I hereby extend a friendly welcome to the members of this Symposium on Physics and Nondestructive Testing. 
Warren J. McGonnagle: Thank you, Mr.Smith. Our next speaker is Mr. Louis Bonnefond of the United States Atomic Energy Commission, Division of Reactor Development.

Louis Bonnefond: On behalf of the United States Atomic Energy Commission, I wish to take this opportunity to welcome you to the Second Physics and Nondestructive Testing Symposium. Mr. Conner has asked me to express his regrets that he could not attend the meeting because of other commitments.

In the few minutes which have been allotted to me, it is somewhat presumptuous to attempt to outline the importance placed upon nondes tructive testing by the United States Atomic Energy Commission and other governmental and industrial organizations. From the standpoint of the Atomic Energy Commission, the organization with which I am most familiar, the operational and structural integrity required of materials and components utilized in nuclear reactors is extremely important. The requirements which have been imposed upon these materials and components have made it necessary for the nuclear industry to depend heavily upon the art and science of nondestructive testing. With this incentive, the Atomic Energy Commission has supported in the past and will continue to support nondestructive testing development programs. It is our objective to not only seek refinements or modifications of existing nondestructive testing techniques, but to investigate and develop new and better techniques which will increase the Commission's, and consequently industry's, nondestructive testing capability.

Permit me to point out a few examples where nondestructive testing has been invaluable in evaluating materials and components to be used in nuclear reactors. The extreme thickness of pressure vessels now being utilized in nuclear reactors has necessitated the development of new techniques for detecting subsurface flaws and imperfections in the material. The quality control of the nuclear fuel element is almost entirely dependent upon nondestructive testing techniques. Nondestructive testing methods are used to determine the core position in the fuel element, to detect subsurface flaws in tubular cladding materials, to determine the homogeneity of the fissile material within the matrix and to determine the core-to-clad bonding. Without nondestructive methods to evaluate the above conditions, it would be virtually impossible to determine the integrity of a fabricated fuel element.

Nondestructive testing is of great interest to a variety of industries, as is evidenced by the attendance at this meeting. Because of its general interest, meetings such as this are held to disseminate information which may be utilized by industry. I know that the information that will be presented during this symposium by our distinguished speakers will be valuable to you. Thank you.

Warren J.McGonnagle: Thank you, Mr. Bonnefond. 


\section{PROGRAM}

SESSION I - PENETRATING RADIATION (9:45 AM - 1:00 PM)

Activation Analysis for Material Testing and Research

*E. A. Burrill - High Voltage Engineering Corporation

J.Hirschfield - High Voltage Engineering Corporation

Neutron Radiography: A Second Progress Report

H. Berger - Argonne National Laboratory

Investigations in Neutron Imaging

H. V. Watts - Armour Research Foundation

SESSION II - FILM (2:00 PM - 5:00 PM)

The Latent Image

A. H. Allard - Eastman Kodak Company

Meaning of the Characteristic Curves

*E. T. Larson - Ansco Corporation

H. F. Nitka - Ansco Corporation

Factors Affecting Radiographic Sensitivity

W. D. Kiehle - Eastman Kodak Company

Reception - Guest Lounge, Building 617 (6:00 PM)

Dinner - Guest Lounge, Building 617 (6:45 PM)

Scientific Lynch Law (8:00 PM - 200 Auditorium)

John W. Campbell, Jr., - Editor, Analog Science Fiction Magazine

SESSION III - ULTRASONICS (9:00 AM - 12:30 PM)

Generation and Detection of Ultra High Frequency Sound Waves in Solids

H. E. Bömmel - Bell Telephone Laboratories, Inc.

(on leave to the University of California)

Ultrasonic Propagation in Deformed Single and Polycrystalline Materials

*F. R. Rollins - Midwest Research Institute

R. R. Rowand - Wright Air Development Center

Mechanical Vibrations in Solid Rods

R. G. Peterson - Argonne National Laboratory

*Author presenting paper 
SESSION IV - OPTICAL AND ELECTRICAL PROPERTIES (2:00 PM 5:00 PM)

Some Optical and Electrical Properties of Materials

R. J. Maurer - University of Illinois

Infrared Physics

H. L. Sachs - Perkin-Elmer Corporation

Thermistors

R.S. Goodyear - Fenwal Electronics, Inc.

Dinner - 203 Cafeteria (6:00 PM)

Under the Arctic with the U.S.S. Skate (8:00 PM - 200 Auditorium)

Lt. Commander Guy H. B. Shaffer - U.S. Naval Submarine School

SESSION V - MATERIALS (9:00 AM - 12:30 PM)

Nondestructive Testing of Reactor Irradiated Metals

T. H. Blewitt - Argonne National Laboratory

Color Radiography

N. S. Beyer - Argonne National Laboratory

Some Design Aspects of Linear Accelerators Used for High Energy X-Ray Production

J. Haimson - Varian Associates

SESSION VI - TOUR OF ARGONNE FACILITIES (1:00 PM - 4:30 PM)

The Zero Gradient Synchrotron

A. V. Crewe - Argonne National Laboratory 


\title{
ACTIVATION ANALYSIS FOR MATERIALS
}

\section{TESTING AND RESEARCH}

\author{
by \\ E. A. Burrill \\ and \\ J. Hirschfield \\ High Voltage Engineering Corporation \\ Burlington, Massachusetts
}

\begin{abstract}
The uses of positive-ion accelerators and neutron sources for material evaluation and analysis are described in terms of basic experimental results obtained on a variety of problems. Surface and volume activation techniques are appraised from data as well as from theoretical considerations. Problems of neutron and radioactivity detection are discussed from the viewpoint of reduction to practice in the industrial laboratory.
\end{abstract}

\section{Introduction}

The technique of activating materials by neutrons or high-velocity ions has broad application in research and industry. Much of the original reported work has been concerned with detailed chemical analysis and the detection of trace concentrations. This emphasis arose from the impressive results with powerful nuclear-reactor neutron fluxes that yield extremely fine sensitivities for many isotopes.

As the activation method has become better understood, its scope of application has gone further than merely chemical analysis. Modern instrumentation and apparatus have reduced the technique to laboratory practice at costs that are compatible with those of other analytical scientific instruments. Particle accelerators can now provide a wide selection of neutron energies and fluxes, as well as a variety of energetic positive ions, for metallurgical, chemical, biological, and industrial processcontrol purposes.

It is now practical to investigate not only chemical content and trace constituents, but also the condition of surfaces, the composition and configuration of crystal and grain boundaries, and the general solid state of matter. This paper describes typical apparatus of value in such programs, as well as a few important techniques and a sampling of provocative applications. 
Activation Apparatus

Several types of accelerator sources for producing neutrons are now available commercially. Their basic characteristics are outlined in Fig. 1. (1) These sources fall into the following general categories:

a. Low flux: neutrons from the D-T reaction; relatively inexpensive: marginally useful thermal fluxes.

b. Moderate flux: neutrons available from both the D-T and D-Be reactions; positive ions with sufficient energy to activate surfaces; broadly useful thermal fluxes.

c. High flux: neutrons available from many nuclear reactions: great versatility for fundamental and applied research; intense thermal fluxes for activation or other radiation effects.

\begin{tabular}{|l|c|c|c|}
\hline Voltage (kv) & $50-150$ & $400-1300$ & $2000-4000$ \\
\hline Accelerator type & transformer & Van de Graaff & Van de Graaff \\
\hline D-T fast flux & $10^{6}-10^{8}$ & $10^{8}$ & $10^{8}-10^{9}$ \\
D-Be fast flux & - & $10^{7}-10^{8}$ & $10^{10}-10^{11}$ \\
Thermal flux & $10^{5}-10^{7}$ & $10^{7}-10^{8}$ & $10^{10}-10^{11}$ \\
Neufron reaction & D-T & D-Be & D-Be \\
\hline
\end{tabular}

Fig. 1. Accelerator Neutron Sources.

Activation techniques often involve fast neutrons as well as thermal fluxes. It is, therefore, not sufficient to examine merely the characteristics of total neutron output from the targets of various accelerator sources of neutrons. Indeed, it is necessary to evaluate the following aspects:

a. Fast neutron flux $\left(\mathrm{n} / \mathrm{sec}-\mathrm{cm}^{2}\right)$ from the D-T reaction, yielding 14-Mev neutrons.

b. Thermal flux, from either the D-T or the D-Be reaction, depending on the bombarding energy.

c. The longevity of neutron-producing targets, as a function of microampere-hours, bombarding energy, and ion-beam diameter. 
Figure 2 shows the neutron yield (n/sec total from the target) from several nuclear reactions as a function of the bombarding energy. It is quite apparent that, for total quantity of neutrons, the D-T reaction is to be preferred at energies below $1 \mathrm{Mev}$. This characteristic has made it

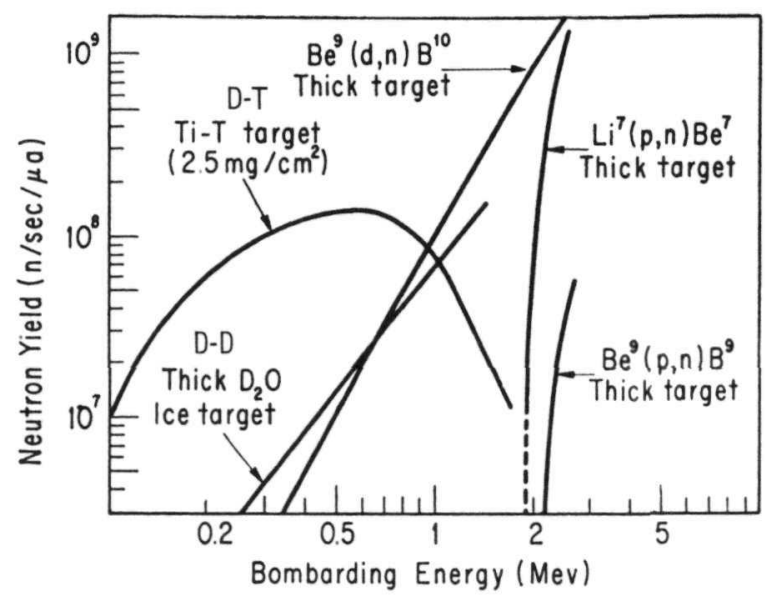

Fig. 2. Experimental Neutron Yields from Different Beams, Beam Energies and Target Thicknesses. possible to design and construct relatively inexpensive, low-voltage neutron sources, but capable of exploiting mainly the D-T reaction.

At higher bombarding energies, the D-Be reaction yield becomes more prolific, with a spectrum of neutrons ranging in energy from about 1 to $6 \mathrm{Mev}$. At $400 \mathrm{kev}$, for example, the output from the D-Be reaction is about $1 / 20$ the output from the D-T reaction. At first glance, it seems apparent that the D-Be reaction would not be particularly useful at these relatively low bombarding energies.

When the thermal flux from these two reactions is compared, however, the conclusion becomes somewhat altered. Figure 3 shows the distribution of the thermal flux, in a paraffin or water moderator, for $\mathrm{D}-\mathrm{T}$ and D-Be neutrons. The relative values of flux are not as important, in this discussion, as the shapes of the flux-distribution curves. The 14-Mev neutrons from the D-T reaction are moderated with less concentration than the 1-6-Mev neutrons from the D-Be reaction. This result arises from the fact that 14-Mev neutrons are less easily slowed down than are 1-6-Mev neutrons.

Fig. 3

Thermal-Neutron Fluxes in Paraffin Depend on Initial Neutron Energy. Thermal neutron yield is plotted as a function of distance from source in moderator. Absolute magnitude of $\mathrm{Li}^{7}$ curve is low by factor of $\sim 2.5$ because smaller moderator was used than for other curves.

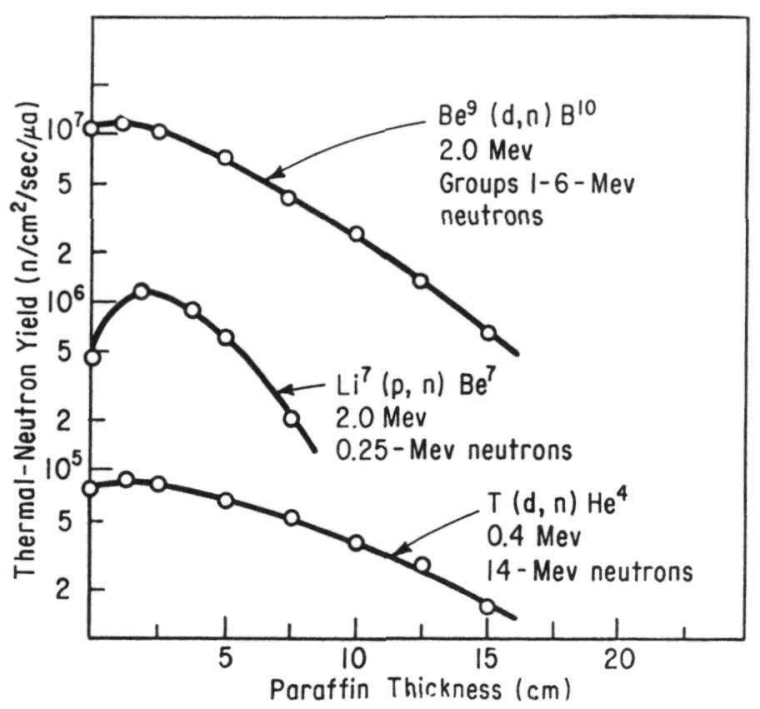


In practice, the following observations are made: at $400 \mathrm{kev}$, a D-T neutron output of $10^{10} \mathrm{n} / \mathrm{sec}$ results in a maximum thermal flux of about $10^{7} \mathrm{n} / \mathrm{sec}-\mathrm{cm}^{2}$ in a paraffin or water moderator; for the same deuteron current at $400 \mathrm{kev}$, the D-Be neutron output is less than $10^{9} \mathrm{n} / \mathrm{sec}$, yet with a thermal flux approximating $10^{7} \mathrm{n} / \mathrm{sec}-\mathrm{cm}^{2}$. The two thermal fluxes are therefore comparable, especially in the light of the following discussion.

The importance of this comparison is centered about the problem of neutron-target life. The target normally used at low bombarding energies consists of tritium gas adsorbed to an evaporated layer of titanium or zirconium. The condition of these targets is strongly dependent on contaminating films, deuteron bombarding energy, ion-beam intensity and diameter, and the distribution of tritium throughout the target. The neutron yield from tritium targets deteriorates with time, as shown in Fig. 4. The yield can be expected to drop to one-half its original intensity in approximately 500 microampere-hours. $(2,3)$ With a defocused beam, some improvement is possible, perhaps to 1000 microampere-hours.

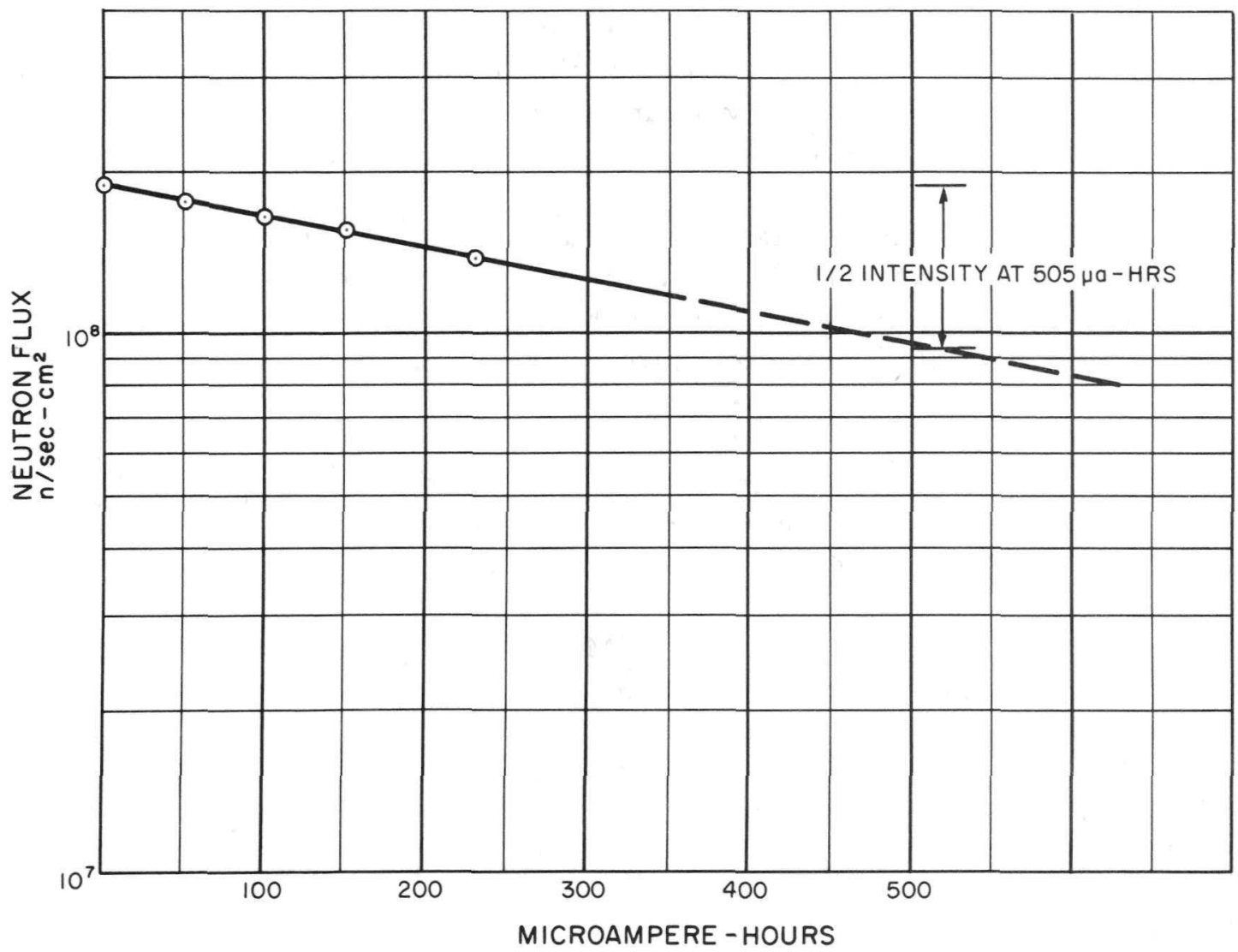

Fig. 4. Tritium Target Life. 
As the bombarding energy is decreased below $400 \mathrm{kev}$, the "halflifetime" can be expected to decrease, not only in microampere-hours, but also in total useful operating hours, because the deuteron current must be increased to maintain the same neutron yield. At very low bombarding energies, furthermore, there is a great possibility of deuteron contamination of the target surface, which gives rise to a weak but interfering D-D neutron output. This effect is not so pronounced with deuteron energies having sufficient penetration into the volume of the target.

The operating half-life of a tritium target, at $400 \mathrm{kev}$, for $10^{10} \mathrm{n} / \mathrm{sec}$, can be expected to be 4 to $6 \mathrm{hr}$. At half this energy, for an equivalent neutron output, the operating life is 30-45 $\mathrm{min}$.

The beryllium target, on the other hand, is made of metal, with es sentially an infinite life. Wherever possible, its use should be encouraged because of its convenience, reliability, and ease of maintenance. There are, to be sure, several neutron-activation reactions that require neutron energies greater than 5 or $6 \mathrm{Mev}$ for satisfactory sensitivities. The occasional use of the tritium target would be required in a practical activation facility. Such a target could be interposed between the deuteron beam and the beryllium target, without breaking the vacuum, for maximum speed and convenience.

As the deuteron energy is increased, up to 1 Mev or so, the intensity of the neutron flux from the D-Be reaction rises much more sharply than the capital cost of the equipment. Since the D-T reaction maximizes at a bombarding energy of about $800 \mathrm{kev}$ with available targets, there is little gain in 14-Mev neutron yield to be obtained, at the same current, with higher energies. The thermal flux from the D-Be reaction, on the other hand, becomes about 10 times more intense at $1.3 \mathrm{Mev}$ than the corresponding flux from the D-T reaction. For routine analytical work with thermal neutrons, a flux of greater than $10^{8} \mathrm{n} / \mathrm{sec}-\mathrm{cm}^{2}$ provides a broad scope of usefulness.

A typical facility, utilizing a 1.3-Mev accelerator, is shown in Fig. 5. In addition to the accelerator and its shielded facility, the following instrumentation and apparatus are recommended:

a. Paraffin or water moderator for thermal-neutron production.

b. A manual or pneumatic sample-handling arrangement to transfer the irradiated samples to the counting array in the most convenient manner; the pneumatic system is particularly needed for those activations with half lives of a few seconds.

c. Radioactivity-detection systems, including scintillation counters in shielded containers, associated photocells, and amplifiers. 
d. Data-interpreting equipment, in the form of single- or multichannel pulse-height analyzers, with read-out accessories such as typewriter, "x-y" plotter, or simple
oscilloscope display.

e. Ion-beam pulsing circuits, to modulate the neutron output for the irradiation of isotopes whose half-lives are less than a few seconds; counting is done in the irradiation area and is synchronized for recording activity
between neutron bursts.

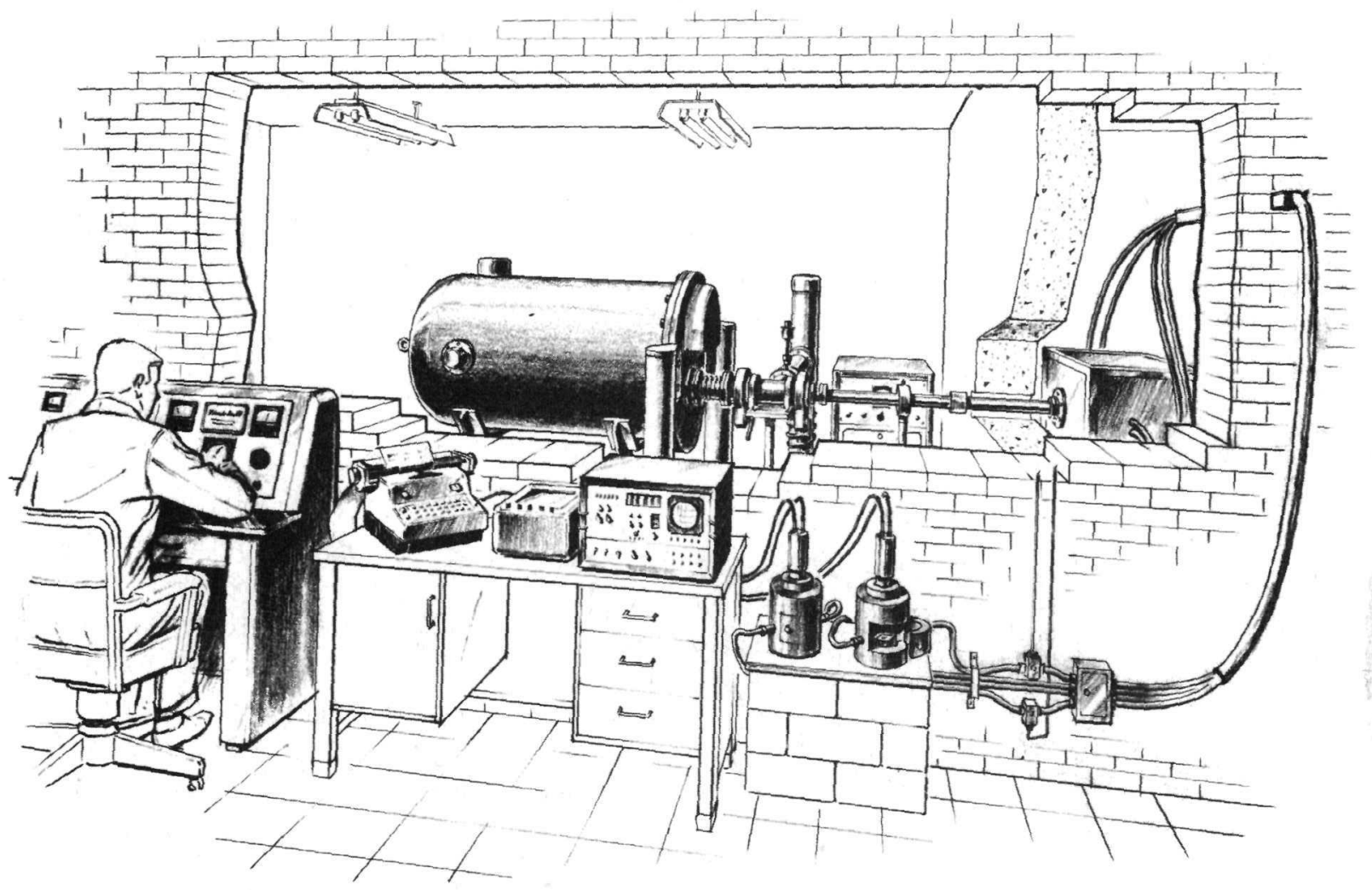

Fig. 5. Activation Analysis Facility.

The design of a facility is governed by end use. For a broad program of materials testing and research, a relatively powerful accelerator, with be equipped with fairly sophisticated and ion-bombardment capabilities, should essing. On the other hand, an extremerion and data procwhich only one or two purposes juemely simple system can be devised for with a single - or dual-channel justify the facility. A small accelerator, cations, such as process control.

Summing up the discussion concerning apparatus, it is important to review in detail the scope of the program under contemplation, so that a satisfactory facility can be obtained. In this connection, consideration must 
be given not only to neutron flux (both fast and thermal), but also to convenience and research economy.

\section{Activation Techniques}

The scope of the activation technique can be enhanced by the availability of a selection of neutron energies at significantly useful fluxes. Thermal neutrons can activate many of the isotopes through simple neutroncapture $(n, \gamma)$ reactions. Some isotopes, however, respond preferentially to fast neutrons, by the $(n, p),(n, \alpha)$, or $(n, 2 n)$ reactions.*

In cases for which certain isotopes respond preferentially to a particular neutron energy, there is a possibility of segregating the activity of interest. The analysis of barium oxide can serve as an example. Figure 6 is a gamma-ray spectrum of the radioactivity induced in $\mathrm{BaO}$ from thermalneutron irradiation. Only the barium peak at $165 \mathrm{kev}$ (from Ba ${ }^{139}$ ) appears. When $\mathrm{BaO}$ is irradiated with 5-Mev neutrons (see Fig. 7), there is again evidence only of barium, but predominantly from the 670-kev peak (from $\mathrm{Ba}^{137}$ ). With 14-Mev neutrons (see Fig. 8), the presence of oxygen is detected by the gamma-ray peaks at 5-6 Mev. The oxygen reaction is $\mathrm{O}^{16}(\mathrm{n}, \mathrm{p}) \mathrm{N}^{16}$; with a maximum gamma peak at $6.2 \mathrm{Mev}$ and two subsidiary peaks due to positron-annihilation radiation.

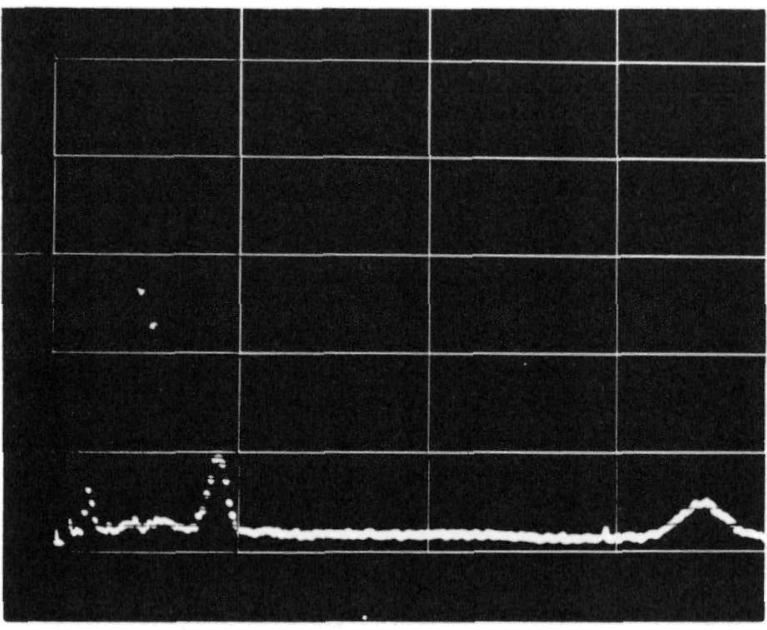

Fig. 6. BaO with Thermal Neutron Activation.

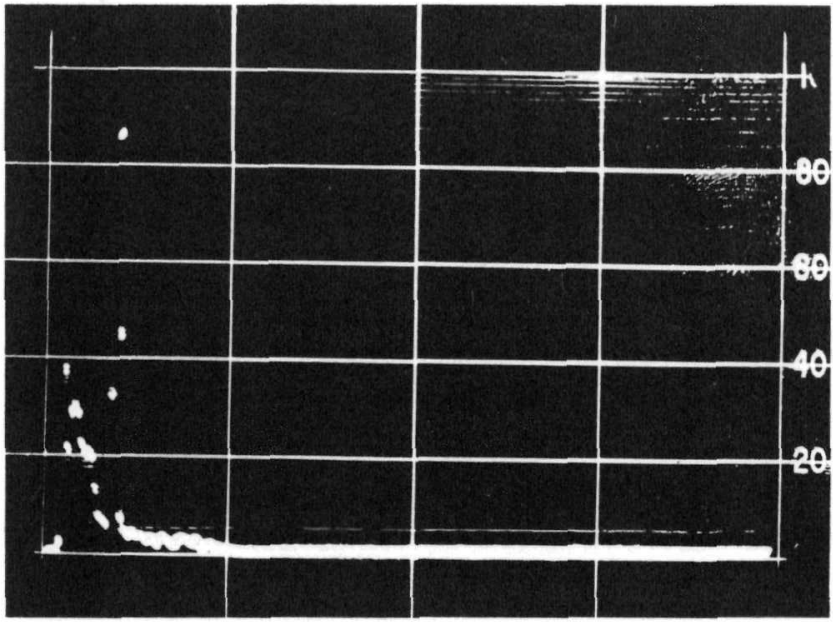

Fig. 7. BaO with 5-Mev Neutron Activation.

*The short-hand notation indicates the particle entering, and the particle(s) or radiations leaving the irradiated nucleus. The $(n, \gamma)$ and $(n, 2 n)$ reactions produce other isotopes of the same element, whereas the $(n, p)$ and $(n, \alpha)$ reactions produce isotopes of elements with atomic numbers one or two lower than the bombarded element. 


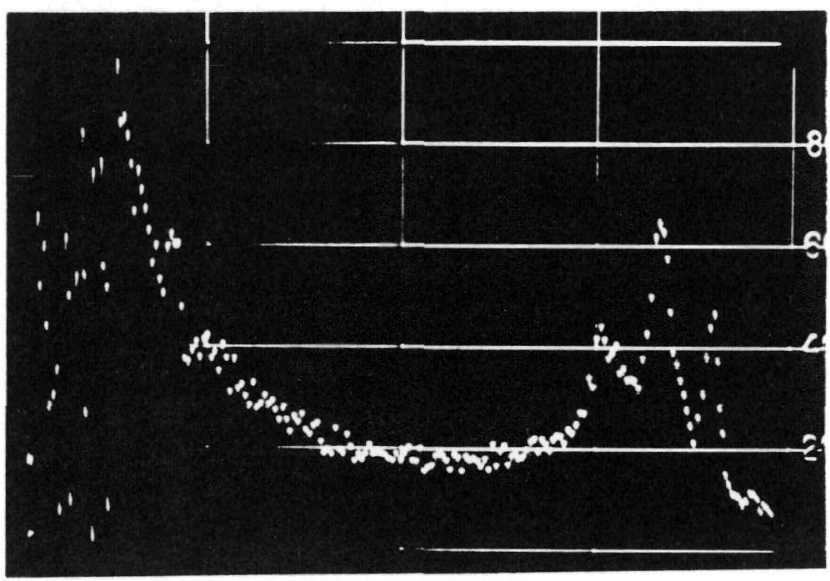

Fig. 8

$\mathrm{BaO}$ with 14-Mev Neutron

Activation.

By difference, the oxygen activity could be isolated from the barium, using very simple counting methods and two irradiations. More accurately, however, the oxygen peaks can be analyzed with complete disregard for the barium radiation, by using a multichannel analyzer, or a single-channel analyzer discriminated in favor of the oxygen radiation.

An interesting interference in this method involves the possible presence of fluorine. Fast neutrons with energies greater than 3 Mev can activate fluorine by the $\mathrm{F}^{19}(\mathrm{n}, \alpha) \mathrm{N}^{16}$ reaction, yielding the same gamma spectrum that is typical of the $\mathrm{O}^{16}(\mathrm{n}, \mathrm{p})$ irradiation. Fluorine can be analyzed in the presence of oxygen, by bombardment with 5-Mev neutrons, because oxygen requires neutrons of $12-\mathrm{Mev}$ energy. Oxygen, on the other hand, cannot be analyzed in the presence of fluorine unless the latter isotope is low in concentration with respect to oxygen, because both elements would become active with $14-\mathrm{Mev}$ irradiation. The fluorine content could be subtracted from the total, from the results of a lower energy bombardment, but only if it represented a relatively small interference.

Intermediate neutron energies can be used to advantage, from P-Li and $\mathrm{P}-\mathrm{T}$ neutron-producing reactions, which yield neutrons in the $100-$ 300-kev range. There are many resonances in neutron cross sections in this particular realm that could provide separable effects for analysis. The P-T reaction is practical only above its $1.02-\mathrm{Mev}$ threshold; the P-Li reaction threshold is at $1.88-\mathrm{Mev}$. Both reactions yield neutron fluxes of a similar order as the D-T and D-Be reactions. By slowing down the energies of these intermediate neutrons with a few centimeters of paraffin moderator, neutrons of a few electron volts are obtained. With a pulsed-neutron beam, synchronized with the detection instrumentation, particular neutron energies (and their effects) can be segregated, by the neutron "time-offlight" method. (4)

In order to use these various neutron-producing reactions in the practical laboratory sense, it is necessary to have sufficient bombarding energy, the convenient selection of proton or deuteron beams, the optional availability of ion-beam pulsing, and a target arrangement that permits shifting from one reaction to another without interfering with the vacuum system. 
Rotating or sliding target arrays within the vacuum are practical and are often used. Flexible bellows couplings permit a lateral shift from one target to another with respect to the ion-beam axis. The major problem under these conditions concerns the target diameter. Where thermal neutrons are of importance, a large target cross section tends to reduce the thermalization of neutrons in the moderator because a large solid angle is presented to the neutrons for escape into the air. An alternative is to utilize a target of small diameter, with an auxiliary hinged target that can be interposed between the bombarding beam and the most utilized target.

Maximum versatility can be obtained with a magnetic beam-switching system to deflect the ion beam into one of several positions. In elaborate laboratories, the beam is often switched into different radiation areas, so that separate experimental set-ups can be prepared independently of the accelerator use in another area.

Another method of activating specific isotopes involves the direct bombardment of samples by protons, deuterons, or alpha particles. Because of the low penetration of these ions, even at energies up to a few Mev,

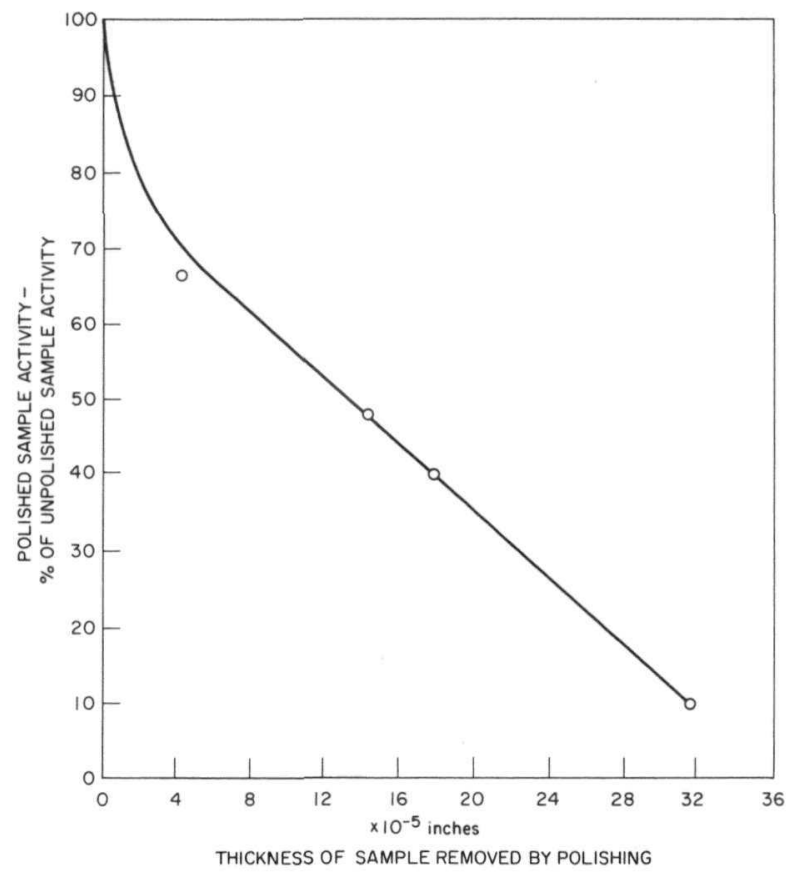

Fig. 9. Distribution, in Depth, of Radioactivity Induced in Stainless Steel by 2-Mev Deuteron Bombardment. irradiation in vacuo is preferred. The induced radioactivity is correspondingly limited to the surface layers of the sample material. As an example of this small penetrability, Fig. 9 shows the distribution of total radioactivity in a steel sample bombarded by $2-$ Mev deuterons. About 90 percent of the activity is contained within a depth of 0.0003 in., and 45 percent lies within $0.000 \mathrm{l}$ in. This distribution of activity was determined by progressively electropolishing the steel sample between measurements. The sum of remanent radioactivity in the sample and accumulated activity in the electrolyte remained constant within 20 percent. No sample activation was attributable to neutrons incidentally produced by the ion bombardment. A similar experiment was conducted in which the wear of steel tool bits was monitored after ion activation. The sensitivity of this technique indicates that a wear of 10-20 microinches could easily be detected from measurements on the worn part.

The effects of ion activation can be somewhat segregated by a proper choice of bombarding energy. For example, chromium $\left(\mathrm{Cr}^{53}\right)$ becomes 
active when bombarded with deuterons of about 500-kev energy; carbon ( ${ }^{12}$ ) is activated by $328-\mathrm{kev}$ deuterons or $460 \mathrm{kev}$ protons; sodium ( $\mathrm{Na}^{23}$ ) requires l-Mev deuterons; iron, on the other hand, is not activated below a deuteron energy of 3-Mev.

Ion bombardment produces sufficient radioactivity to permit autoradiography of the irradiated surface. With $5 \times 10^{13}$ deuterons of $2-\mathrm{Mev}$ energy (about $10 \mu \mathrm{a}-\mathrm{sec}$ ), a total of $10^{5}$ disintegrations resulted from the irradiation of a steel sprocket tooth. It was necessary to wait for about $16 \mathrm{hr}$ for the radioactivity to "cool down" so that the counting system would not saturate. This technique should be useful in the study of preferential migration or diffusion of alloy constituents.

Through the phenomenon of elastic ion scattering from surfaces, a momentum analysis of the ions scattered at a particular angle reveals a quantitative and qualitative analysis of the surface composition. The technique was originally applied to a study of internal gun-barrel surfaces (to determine the corrosive effects of gunpowder), (5) but more lately the method has been extended to an analysis of clays and sedimentary rocks. (6) Ion energies above $1 \mathrm{Mev}$ are needed for this type of analysis. Indeed, there are advantages to the use of ions heavier than the hydrogen isotopes for a more significant momentum transfer between bombarding ion and target nucleus.

A momentum analyzer, such as a broad-range magnetic spectrograph or a spectrometer, is useful for the detection of elastically scattered protons. Furthermore, this type of analyzer permits the observation of nuclear-reaction products from $(p, \alpha),(d, p)$, and similar charged-particle reactions. In the bombarding-energy range of l-2 Mev, the use of nuclear reactions is advantageous for the detection of light nuclei. On the other hand, the sensitivity of the scattering method increases with heavier nuclei.

As the bombarding energy is increased, above the 1-2-Mev range, it becomes increasingly practical to bring the ion beam out into the atmosphere through a very thin metallic window. For example, gas analyses have been studied with 3-Mev deuterons, in which the gas samples were allowed to flow past a thin nickel window. (7) Radioactive isotopes thus formed (e.g., oxygen from nitrogen bombardment and nitrogen from carbon bombardment) could then be introduced into chemical compounds as short-lived tracers to investigate equilibrium constants and methods of molecular bonding. This general irradiation technique is also applicable to surface analysis of metallic or organic solids, without breaking the vacuum. One problem involves the presence of surface contaminants, such as occluded gases from the atmosphere or organic films from manual handling. Careful cleaning of the surfaces is extremely important. 


\section{Activation Instrumentation}

A single-purpose activation facility, utilizing one of the irradiation methods described above for the analysis of a few selected isotopes, can be designed with relatively simple instrumentation. The accelerator can be chosen according to the neutron energy and flux requirements for the particular activations. The counting system can be adapted for the specific radiations involved. A single-channel pulse-height analyzer can be used to monitor the important gamma peaks. In fact, the sensitivity of counting can be adjusted to permit a direct read-out in terms of weight percent of some similar parameter. Such a facility could be contemplated for in-line process control.

The calibration of this single-purpose activation analyzer can be accomplished internally, by comparing the unknown concentration of one isotope in terms of the predictable concentration of another isotope in the alloy or compound. Two channels of analysis would be adequate for this type of control. Alternatively, simultaneous irradiation of a known sample would provide a check on the accuracy of the method. Two counters would be required for this case.

Figure 10 is a display of two activities being counted simultaneously. Although this illustration is derived from the oscilloscope trace of a multichannel analyzer, the peaks of interest could as readily have been monitored by two channels. This display is the gamma spectrum of sodium fluoride, the high-energy peaks being attributable to the nitrogen activity from $\mathrm{F}^{19}(\mathrm{n}, \alpha) \mathrm{N}^{16}$ (compare, on an expanded scale, with Fig. 8). The low-energy peak is due to sodium. By comparing the two $6-\mathrm{Mev}$ peaks, a ratio analysis of the fluorine content can be accurately obtained.

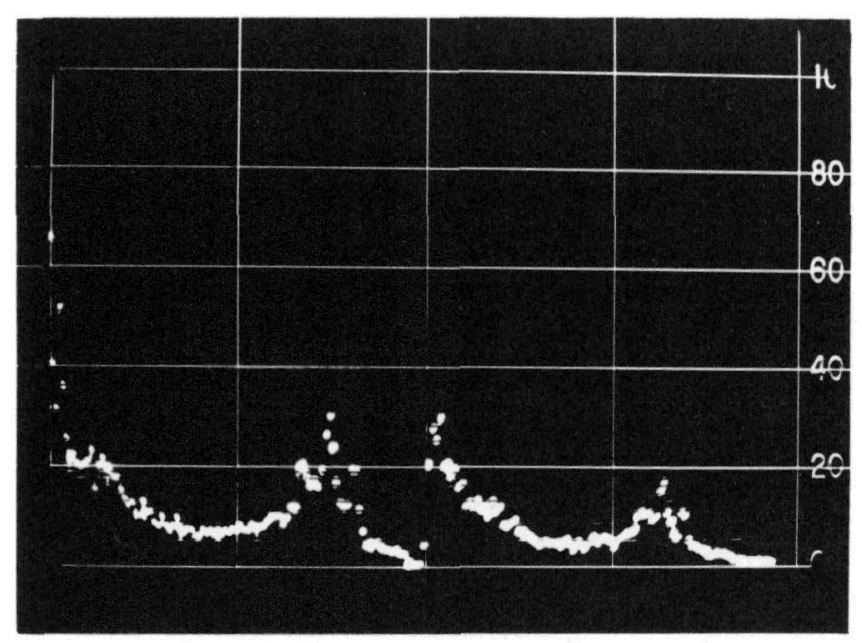

Fig. 10. NaF - 5-Mev Neutron: Ratio Analysis. 
For a versatile activation facility, on the other hand, an acquaintance is necessary with the many methods now available for calibrating neutron flux, for radiation detection, for interpreting and processing the data, and for handling the samples between neutron source and counter.

It is obviously important to have an accurate measure of the number of neutrons involved in a particular activation. The time of irradiation at a particular flux determines the percent saturation of activity (see Fig. ll). As the sample is bombarded, it progressively decays according to its radioactive half-life. Saturation activity occurs at an irradiation time less than 10 half-lives. For practicality, however, bombardment times equivalent to 2 half-lives yield 75 percent saturation. In the case of very long-lived isotopes, the radioactive yield is roughly proportional to irradiation time, as well as to flux intensity. For very short-lived isotopes, bombardment times depend on operator convenience, because saturation may occur in less time than is feasible to control. Longer irradiation does not matter, since saturation has been reached.

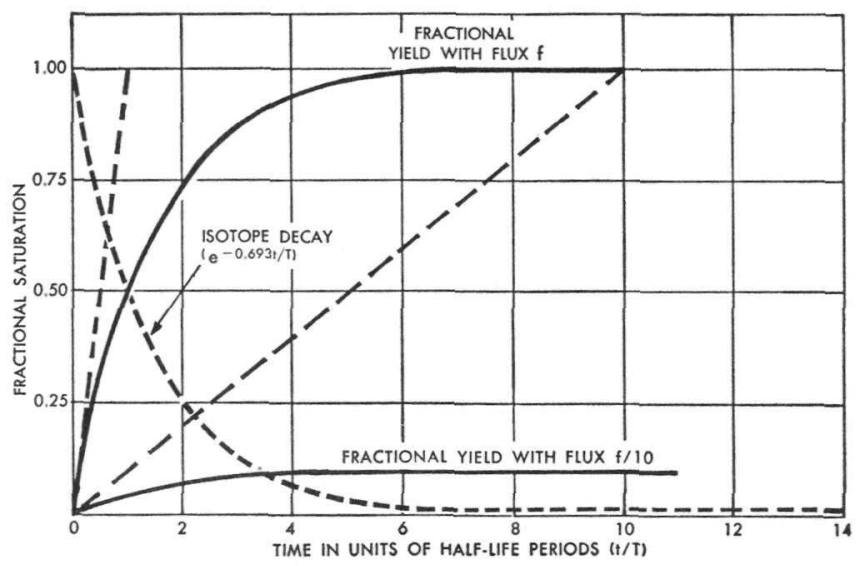

Fig. 11

Growth and Decay of Radioactive Isotope with Neutron Bombardment.

During the irradiation period, the neutron-flux variation governs the level at which saturation activity is obtained. The flux can change during irradiation for a number of reasons, including accelerator-voltage instability, ion-beam current fluctuations, and neutron-target contamination or deterioration. The first two variables can be controlled or monitored directly by electrical or electronic means. There is no guarantee, however, that the flux remains as constant as the accelerator parameters. For this reason, flux monitoring or calibration is important.

A moderated $\mathrm{BF}_{3}$ "long" counter is effective for directly measuring the fast or thermal neutron flux. It can be calibrated from standard radioactive sources of neutrons. The variations in neutron flux during irradiation can be recorded by strip chart. For long-lived isotope production, the total number of neutrons is an adequate measure. For short-lived species, however, large changes in flux during irradiation introduce errors in data interpretation. 
The ratio-analysis technique mentioned above is quite accurate, because the 2 samples are exposed to the same irradiation conditions and are counted simultaneously. Fluctuations in flux, or even uncertainties in irradiation time, affect each sample similarly.

The use of calibrated thin metallic foils, such as of gold, silver, or indium, is routine for thermal-flux monitoring. Depending on irradiation time, the proper foil choice would give an accurate estimate of the neutron flux; for example
$\mathrm{Au}^{198}$
2.7 days
0.4-Mev $\gamma$
In $^{116}$
$54 \mathrm{~min}$
1.3-Mev $\gamma$
$\mathrm{Ag}^{108}$
$2.3 \mathrm{~min}$
$\mathrm{Ag}^{110}$
24 sec
$\left.\begin{array}{l}2.8-\mathrm{Mev} \beta \\ 2.8-\mathrm{Mev} \beta\end{array}\right\}$ no $\gamma$ ray

The activity in the foils can be measured at the same time as the activity in the unknown sample when 2 counters are available, thus providing a basis for calculating the concentration of the unknown. The sample and its flux standard can be measured sequentially if only one counter is at hand, with corrections applied for the decay of the standard. Fast-neutron fluxes can be similarly calibrated through the use of "threshold detectors," foils of isotopes whose activation by fast neutrons occurs only above a certain energy threshold. Sulfur and silicon are among the isotopes normally used.

A simple and effective monitor of a 14-Mev neutron flux consists of an ordinary Geiger-Muller tube which continuously counts the induced nitrogen activity from the bombardment of the oxygen in the target-cooling water. Provided that the water flow is consistent, this method is adequate for many general applications, despite the poor efficiency of the $\mathrm{G}-\mathrm{M}$ tube for counting the 6.2-Mev gammas from nitrogen.

Gamma-ray spectroscopy has become a standard method of meas uring radioactivity, with scintillation crystals and multichannel analyzers. For certain radioisotopes, however, beta rays represent the only radiations emitted; consequently, G-M tubes of appropriate configuration are needed. The information obtained by this general technique is not usually spectrum analyzed. Furthermore, the method is usually confined to specific isotopes for which there is no particular interference by other elements in the sample. Beta-ray spectrometers are available, but their use is not generally as straightforward as in the gamma-ray case.

The preparation and handling of samples depend on the method of irradiation. Neutron activation analysis requires little or no sample preparation; in fact, it is considered to be a nondestructive test. For quantitative analysis, the sample should be small (a few grams) so that the entire volume is exposed to the same flux of neutrons, and also so that there is a 
minimum of self-shielding from its own gamma rays. When 2 large samples are simultaneously irradiated (standard and unknown), however, this limitation does not pertain, provided that the samples are of identical geometry.

For most activations, manual handling is adequate because the decay half-lives are sufficiently long to permit slight delays between cessation of irradiation and beginning of counting. The important parameter is the irradiation time and, especially, the instant the irradiation was stopped. All decay curves are plotted with this instant as the ordinate, so that the decay can be extrapolated back to "zero" counting time.

In cases where the half-life is extremely short, e.g., fluorine or oxygen with 7-sec half-lives, a rapid transfer is needed between neutron source and counting system, to maximize the counting rates. A pneumatic tube or "rabbit" system can shuttle the samples back and forth, on a recycling basis, to improve the counting statistics for better detection sensitivity or accuracy. Two "rabbits" are to be preferred, to carry both the unknown and the standard。( $(8,9)$

Data interpretation is a complex subject in itself, but the mechanics have been greatly simplified by the advent of the multichannel analyzer and its associated read-out and computer devices.(10) For some analyses, it is sufficient to observe the approximate values from irradiation directly from an oscilloscope display. This may be especially true in the case of ratio analysis, or where the qualitative detection of particular isotopes is involved. More accurate appraisal of data can be obtained by utilizing typewriter readout, in which the actual accumulated counts in each "energy bin" of the analyzer are recorded. An alternative is the use of an "x-y" plotter, in which the same type of information is transferred to graph paper. For informal, or informative, records, the Polaroid photograph of the 'scope trace is adequate.

The data, as displayed and stored in the analyzer, can be treated with special techniques to amplify or clarify the information obtained. Background can be subtracted by utilizing one-half the analyzer channels for sample counting and the other half for background observation. The latter can be electronically subtracted. The interference of very prominent iso-topes can be subtracted or "stripped" from the display spectrum through the use of standard punched tapes on which the known spectrum is recorded. By normalizing the standard spectrum with the height of the peak in the sample spectrum, a more significant display of the important activity can be obtained. $(2,11)$ 
Conclusion

The applicability of the general activation technique is limited mainly by the available laboratory facilities and the ingenuity of the research and analytical personnel associated with them. The technical literature is progressively increasing as the understanding of the basic concepts of the method becomes more widespread. The commercial availability of accelerator sources of neutrons from several manufacturers has done much to initiate industrial interest and to introduce solutions to bothersome problems.

It becomes obvious, however, that the neutron source itself is only a fraction of the total facility. Because industry is generally not closely acquainted with the principles of nuclear physics and radioactivity, it is necessary to consider custom-built packages of apparatus and instrumentation which can cope with either a limited selection or a broad scope of analytical problems. Furthermore, it is important to provide the use of demonstration irradiation facilities to interested laboratories, so that solutions or techniques for particular problems can be sought. These preliminary demonstrations of feasibility can lead most effectively to the proper component choice in an activation package.

References

1. E. A. Burrill and M. H. MacGregor, Using Accelerator Neutrons, Nucleonics, 18 (12), 64 (1960).

2. V. P. Guinn, Neutron Activation Analysis, International Science \& Technology, Prototype Issue, p. 74(1961).

3. J. H. Coon, "Targets for Production of Neutrons," Fast Neutron Physics, Part I, Interscience Pub. Co, N. Y. (1960) p. 695.

4. R. H. Anderson, T. I. Taylor, and W. W. Havens, Chemical Analysis by Resonance Absorption of Neutrons, USAEC CUD-91 (1951).

5. E. A. Burrill, Scientific Application of Particle Accelerators to Nondestructive Testing, USAEC Symposium on Nondestructive Testing, Argonne National Laboratory (Oct. 5, 1960); to be published in Materials Research and Standards (late 1961 or early 1962).

6. R. F. Sippel and E. D. Glover, Sedimentary Rock Analysis by Charged Particle Bombardment, Nuclear Instruments and Methods, 9, 37 (1960).

7. F. Amiel, Weizmann Institute, Rehovoth, Israel (informal discussion, 1960).

8. Oxygen Yields to Activation Analysis, Chem. \& Eng. News, p. 58 (Apr.11,1960). 
9. O. U. Anders, Determination of Fluorine by Neutron Activation, Anal.Chem., 32,1368(1960).

10. V.P.Guinn and C.D. Wagner, Instrumental Neutron Activation Analysis, Anal. Chem., 32, 317 (1960).

11. O. U. Anders and W. H. Beamer, Resolution of Time-dependent Gamma Spectra with a Digital Computer and Its Use in Activation Analysis, Anal. Chem., 33, 226 (1961).

\section{Discussion}

\section{ACTIVATION ANALYSIS FOR MATERIAL} TESTING AND RESEARCH

D.R. Green, General Electric, Hanford Atomic Products Operation: We are interested in detecting quantitively 100 ppm hydrogen in solid Zir caloy-2 samples. Do you know of any methods of using neutrons produced with an accelerator to apply to hydrogen analysis of small metal samples?

E. A. Burrill: Hydrogen is basically a problem. But one possible way of attacking this problem in the 100-ppm range might be through the concept of neutron transmission. This might work because hydrogen is also an extremely prolific scattering agent for neutrons. There was some experimental activity conducted by Havens and Taylor* of Columbia University some years ago in which they used resonance neutrons of a few electron volts in energy to measure the hydrogen content in various solids in which the transmission of the neutrons in the matrix was fairly good, but in which the scattering from the hydrogen was extremely prolific also.

D. R. Green: You mentioned that you could detect conditions at grain boundaries, and I was wondering what you were referring to there?

E. A. Burrill: This work** was not done in our own organization but has been reported elsewhere. In this particular case, neutrons from a reactor were used in the experiment, but the method is certainly applicable to charged-particle bombardment in which metallurgical samples were prepared so that there is a cleavage along the actual boundary of the crystal. After use of a preferential etching system, the grain boundary could be analyzed for its radioactive content. This could also be done by a surface

*Ed. Note: Taylor, T. I., and Havens, Jr., W. W., Neutron Properties, Interactions, Velocity Selection, Nucleonics, 5, 10 (December, 1949).

**Ed. Note: Schultz, W. W., Grain Boundary Segregation Studies by Activation, General Electric Paper No.171 (1959), Pacific Area National Meeting ASTM, San Francisco (Oct. 11-16, 1959). 
bombardment with ions to make the actual interface between crystals radioactive. Another way of doing this is to consider the radioactivity of a metallurgical sample that has been polished, for example, and to then make an autoradiograph of the radioactive area. This reveals the structure of the interfaces between the crystals and, if there is a preferential distribution of components in the alloy along the grain boundaries, these would show up in the autoradiograph itself. technique?

D. R. Green: Could you study dislocations by this same sort of

E. A. Burrill: I don't know about that. I'm not a metallurgist, and I'm a little afield at the present time on that subject.

S. Z. Mikhail, Kansas State University: In the ratio analysis would you determine the ratio from heights of the peaks or from the areas under the peaks?

E.A. Burrill: It depends upon whether you're using a single-channel analyzer or a multichannel analyzer. To be most effective one would want to look at the areas under the peaks with the multichannel analyzer.

S. Z. Mikhail: How reproducible is this?

E.A. Burrill: Reproducibility of a percent or so is possible with such a technique.

S. Kaufman, Argonne National Laboratory: You have mentioned the technique of studying surfaces by analyzing the momentum of the recoil charged particles. I am wondering if in this technique you're limited to very thin samples or whether you can actually apply it to thick samples met practically. There is going to be energy degradation as the protons penetrate into the sample and slow down, and this may give a background that makes your results useless. Would you comment on that?

E. A. Burrill: There is a good paper*on this particular technique from the Stanford Research Institute in which 2-Mev protons were used for this momentum analysis. If, for isotopes, we look at some measurement of intensity, either spectrometer current or the scintillationcounts, as a function of the momentum of the particles, we will have an approximate measure of the energy, which will also be an approximate measure of the atomic number if it is correlated in the correct sense. If you're looking at something that resembles steel, for example, you'll find that, if we had nothing but iron, we get the curve shown in Fig. 12. This comes as a consequence

*Ed. Note: Rubin, S., Passell, T. O., and Bailey, L. E., Chemical Analysis of Surfaces by Nuclear Methods, Anal. Chem., 29, 736 (1957). 


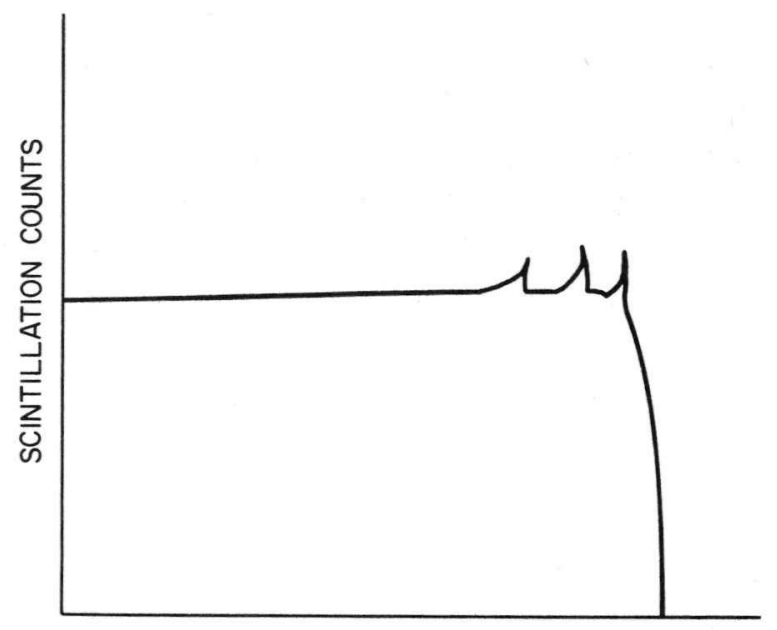

MOMENTUM OF PARTICLES

Fig. 12. Scintillation Counts as a Function of the Momentum of the Particles. of the ions of a monochromatic energy penetrating into the volume of the steel, so you do get essentially a background that is rather immutable. But if there are components in this iron matrix, you'll find that they will reveal themselves as peaks above this background and these peaks are indeed measureable. Their position in this regard is a measure of their atomic weight, and the amplitude of the peak above the background is a measure of either the thickness or the quantitative amount of that particular isotope that is present. It is really quite a delicate method as far as revealing information concerning the surface of the material. Surfaces as thin as a few microns can be analyzed in this manner. This analysis has been also used in the study of tenuous gases. Stanford did some work on the problem of smog analysis by means of this particular technique.

B. Ostrofsky, American Oil Company: I wanted to make one suggestion in answer to a question about determining concentration gradients in metallurgical specimens. There is another nondestructive technique, the electron-beam micro-analyzer technique, which I think would give a more direct solution to this type of problem.

P. E. Dempsey, U. S. Steel: Can we, with this system, look forward to a means of analyzing steel for oxygen with advantages over our present conventional analysis?

E. A. Burrill: I think there's a good possibility of this. It's a quick system. The sample requires very little preparation and the analysis takes a very short period of time - it's all over in a matter of a few seconds. It is something that I think could be a useful process-control technique if there is no fluorine in the same steel.

P. E. Dempsey: Did you say that you used a beam of charged particles to look at grain boundaries?

E. A. Burrill: Yes. If you are studying grain boundaries, it is possible to use charged particles by radiating the surface of the metallurgical sample with the charged particle so that you are observing the surface effect rather than the volume effect. This would be the case if you used neutrons which would penetrate into the depth of the sample. 
W. Z. Leavitt, Watertown Arsenal: I'd like to comment on the question concerning the problem of degradation in fixed samples. Really what you're doing is looking at step functions, and Rubin and others have answered this problem quite easily. Mr. Burrill has shown that you have a low-Z surface on a high- $Z$ matrix. This situation presents quite a different problem than when you have homogeneous impurities. Now you'll end up with steps. I think the answer to the question is that degradation is not a problem. What you're doing is getting your information from the original surface layers, and these give you the steps. The big problem is the presence of an impurity that can give you a higher energy capable of wiping out what you're looking for .

E. A. Burrill: That's right. Thank you, Dr. Leavitt. 
NEUTRON RADIOGRAPHY: A SECOND PROGRESS REPORT(1)

\author{
by \\ Harold Berger \\ Metallurgy Division \\ Argonne National Laboratory \\ Argonne, Illinois
}

\begin{abstract}
This discussion will be a report on the progress made on our investigation of neutron radiography since the first Symposium on Physics and Nondestructive Testing, October 1960. The problems of the production of the neutron image will be discussed. The emphasis will be on the characteristics of many of the photographic imaging methods which can be used for neutron radiography.
\end{abstract}

\title{
Introduction
}

Because relatively little has been published previously on the subject of neutron radiography, $(2-5)$ it seems appropriate to begin this discus sion by restating the reasons for the interest in this potentially useful method for nondestructive testing. This raison d'etre for neutron radiography comes about because the relative neutron absorption of materials is different from the relative absorption of these materials for X rays. Mass absorption coefficients of the elements for thermal neutrons present a random picture if they are examined in terms of regularly increasing atomic number, whereas the mass absorption coefficients for X rays increase with some regularity if they are similarly examined. $(4,5)$ If there is any pattern at all to this examination of thermal neutron mass absorption coefficients versus atomic number, it is that light elements have high absorption and heavy elements have low absorption for thermal neutrons. This reversal of absorption characteristics of materials for thermal neutrons as compared with $X$ rays, and the overall random pattern for thermal neutron absorption combine to make neutron radiography a potentially useful technique for nondestructive testing. Because of these absorption differences, neutron radiography should be useful in many inspection problems which present difficulties by X-ray techniques.

The previous statements have specified that neutrons in the thermal energy region have the absorption characteristics which make neutron radiography attractive. Actually, many of these absorption differences 
continue, for increasing neutron energy, through the epithermal- and slowneutron energy ranges. In the fast-neutron energy region, however, most of these differences in neutron absorption have appreciably diminished, making the use of fast neutrons relatively unattractive for general radiographic applications. This present discussion, with only a few exceptions, will be limited to the use of thermal neutrons.

One other limitation to this report is that only photographic methods of detecting the neutron image will be discussed. The reader desiring further information on other methods is referred to brief reviews of nonphotographic neutron image detection methods in the literature. $(1,6)$ Since photographic emulsions alone are influenced very little by neutrons, (7) intermediate materials which emit some photographically detectable radiation upon neutron bombardment are frequently used to intensify the photographic response of film to neutrons.

Two different exposure techniques can be used. In one, the direct exposure method, the photographic film and converter material are exposed to the neutron beam together. In the other, the transfer method, only a screen is exposed to the neutron beam. This radioactive, image-carrying screen is then placed next to photographic film after the neutron exposure is completed. The film is exposed by the radioactive decay radiation of the screen.

This discussion will be concerned with a comparison( 8 ) between many of the useful converter materials, primarily with regard to photographic speed and qualities of image sharpness. Explanations for some of the observations made concerning these imaging methods will be offered.

\section{Experimental Procedures}

\section{A. The Neutron Source}

A beam of neutrons which contains a very small gamma intensity is one which would be most useful for neutron radiography. This follows from the fact that neutron radiography is of interest because the absorption of neutrons in materials is differentfrom that of Xradiation. Therefor,e, a radiographic image due to gammas superimposed on a radiographic image due to neutrons would be expected to lessen the usefulness of the neutron radiograph. It follows, then, that a neutron source having a high yield of thermal neutrons and a low yield of gamma radiation is desirable for neutron radiography.

A source of thermal neutrons having this feature has been available for the study reported here, and has been used for most of the data to be given. This neutron beam is taken from the neutron spectrometer used for neutron diffraction work(9) at Argonne's CP-5 reactor. A schematic diagram is given in Fig. 1 . 


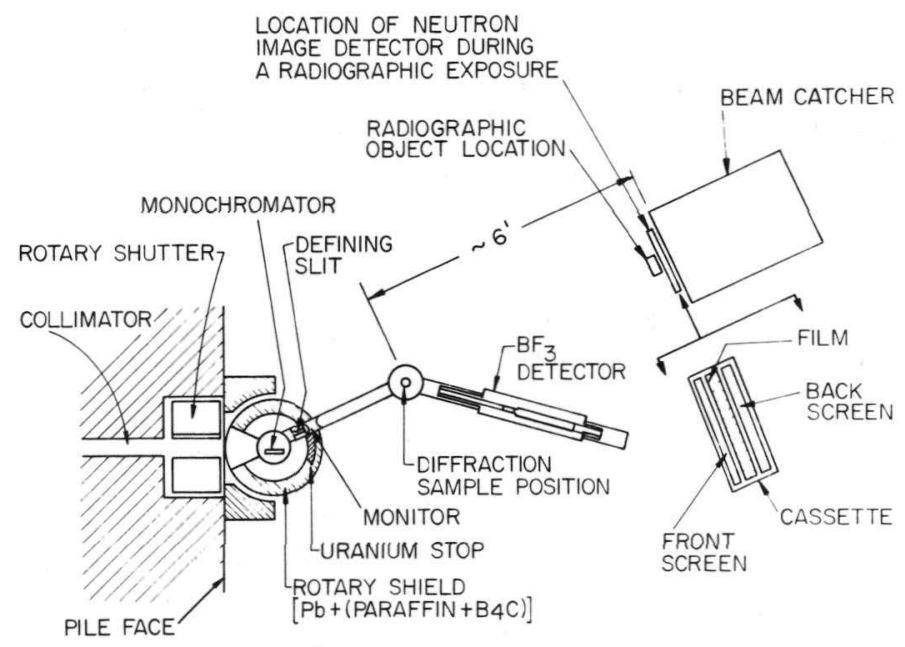

Fig. 1

Schematic Drawing of Neutron Spectrometer Showing Arrangement Used for Neutron Radiography

The monochromatic beam from the monochromatizing crystal emerges through the defining slits and the beam monitor toward the diffraction sample. The portion of the neutron beam which goes through, or around, the diffraction sample enters a beam catcher some distance away. It is this beam, normally unused, which has been employed for this study.

By the time the beam reaches the beam catcher, the relatively low contrast image of the diffraction sample is usually not detectable. The beam is somewhat oval shaped and has the approximate dimensions of $2 \frac{1}{2} \times 3 \frac{1}{2}$ in. A central portion, about $\frac{1}{2} \times 1$ in., contains the maximum beam intensity. At the beam catcher location, this maximum intensity has been determined to be $3 \times 10^{5} \mathrm{n} / \mathrm{cm}^{2}$-sec by means of a gold foil activation method. The neutron wavelength used for the majority of these tests was $1.05 \AA$.

The gamma intensity in the beam is very low insofar as its photographic effect is concerned. This has been confirmed by the facts that direct neutron exposures of very light objects could easily be obtained and that exposures of $\mathrm{KK} X$-ray film to the beam filtered by $\frac{1}{4}$-in. boral sheet yielded no detectable film darkening with exposures as long as $20 \mathrm{~min} .(8,10)$ By comparison, direct neutron exposures with $\mathrm{KK}$ film were normally obtained in the order of 4 min or less.

B. Photographic Detection Methods

Although standard photographic film alone can be used to detect neutrons, (7) much faster, and in many ways more satisfactory, detection of a neutron image can be accomplished by using various converter materials in conjunction with photographic emulsions. (8) The converter materials, upon neutron bombardment, emit some photographically effective radiation. Typical reactions include $\mathrm{B}^{10}(\mathrm{n}, \alpha) \mathrm{Li}^{7}, \mathrm{Cd}^{113}(\mathrm{n}, \gamma) \mathrm{Cd}^{114}$, and many 
reactions which result in radioactive materials (materials such as rhodium, silver, indium, and gold are commonly used). A more complete description of many of the useful reactions for detecting neutron images has been given elsewhere. $(1,8,10)$

For the metal screens used in the direct-exposure method, it should be pointed out that, whether the screens become radioactive or not, a considerable portion of the film exposure appears to be caused by prompt $(n, \gamma)$ radiation from the screen material.(8) This, along with the high cross sections for many of the very short half-life activities, (1) helps account for the fact that most of the film exposure occurs during and very shortly after the neutron exposure, at least for the exposure conditions used for these tests.

One other comment concerning direct-exposure materials is that it has been found much more effective to combine those materials (such as $\mathrm{B}^{10}$ and $\mathrm{Li}^{6}$ ) which emit alpha radiation upon neutron bombardment with a phosphor, so that light, rather than alpha radiation, blackens the film. (2) Three such scintillators have been studied in this investigation. One is a $\mathrm{B}^{10}$-enriched $(92 \%)$ boron polyester type containing $\mathrm{ZnS}(\mathrm{Ag})^{11}$ such as that described by Sun. (12) The other two are LiF-ZnS(Ag) mixtures, such as the one described by Stedman (13) and the similar one employing the modifications made by Shull. (14)

In the direct-exposure procedure normally used in this investigation, the converter screen and the photographic film were placed in an aluminumfront, spring-loaded X-ray cassette. The object being radiographed was attached to the cassette front and the combination was then placed in the exposure position(15) shown in Fig. 1. Exposure times were controlled by means of a manually operated boral-sheet shutter.

In the transfer method used, the metal screen which would eventually carry the radioactive image was normally supported and exposed within the aluminum-front cassette. After the neutron exposure was completed, the foil was transferred to a film-loaded cassette in order to complete the photographic exposure. The transfer of the radioactive screen to film could usually be accomplished within $30 \mathrm{sec}$ of the end of the neutron exposure of the screen.

For the most part, commercially available, double-emulsion X-ray films were used. Development was done in Kodak Liquid X-ray Developer $\left(68^{\circ} \mathrm{F} \pm \frac{1}{2}^{\circ} \mathrm{F}\right)$ for $5 \mathrm{~min}$, without agitation. Density measurements were made with a MacBeth Ansco Densitometer (Model 12A) having a density range of 0 to 6.0 . 


\section{Experimental Results}

A. Direct-exposure Method

A study had previously been made to determine the thicknesses of metal screens which yield the best photographic speed for each of several metals in a double-screen technique. $(1,8)$ In this method, the film is sandwiched between two metal screens during the neutron exposure. The metals studied in this manner, and the resultant thicknesses of metal screens which yielded the best neutron photographic speed for a given neutron exposure are given below. For cadmium, the best screen combination was 10-20, where the numbers are the screen thicknesses in mils, of the screen closest to the neutron source and the back screen, respectively. In terms of a similar notation, best speed results were obtained with 10-10 screens of rhodium, with 20-30 screens of indium, and with 18-18 screens of silver. In each of these cases, the screen thicknesses were chosen at the point where further increases in screen thickness produced little or no speed improvement.

This work has now been expanded to determine the thickness of a single screen and the photographic film orientation to use for best photographic results. In most cases, the use of a single metal screen rather than a double screen does involve a sacrifice of about a factor of two in speed but, for many applications, the improvement in image sharpness justifies this sacrifice. An overall comparison of photographic speed for the materials studied is given in Table I.

TABLE I

RELATIVE PHOTOGRAPHIC SPEED FOR SEVERAL DIRECT-EXPOSURE NEUTRON-IMAGE DETECTION METHODS

\begin{tabular}{|c|c|c|}
\hline $\begin{array}{l}\text { Converter Material and } \\
\text { Screen Configuration }\end{array}$ & Film Type & Relative Speed ${ }^{a}$ \\
\hline $\begin{array}{l}B^{10} \text {-loaded scintillator as back screen } \\
\text { Double rhodium }{ }^{C} \text { screens }(10-10) \\
\text { Double gadolinium } \\
\text { Double indium }{ }^{C} \text { screens }(0.5-2) \\
\text { Double cadmium screens }(10-20) \\
\text { Double silver }{ }^{C} \text { screens }(18-18) \\
\text { Single gadolinium }{ }^{d} \text { as back screen (2) } \\
\text { Single cadmium as back screen (10) } \\
\text { Single rhodium }{ }^{C} \text { as back screen (10) } \\
\text { Single indium }{ }^{C} \text { as front screen (20) } \\
\text { Single silver }{ }^{C} \text { as front screen (15) }\end{array}$ & $\begin{array}{l}\text { F } \\
\text { KK } \\
\text { KK } \\
\text { KK } \\
\text { KK } \\
\text { KK } \\
\text { KK } \\
\text { KK } \\
\text { KK } \\
\text { KK } \\
\text { KK }\end{array}$ & $\begin{array}{l}26 \\
1.4 \\
1.1 \\
1.1 \\
1.0 \\
0.8 \\
0.75 \\
0.67 \\
0.62 \\
0.5 \\
0.35\end{array}$ \\
\hline
\end{tabular}

${ }^{a}$ The relative photographic speed was obtained by comparing film densities and relative exposures for each detector subjected to a similar neutron exposure. The neutron intensity used was $3 \times 10^{5} \mathrm{n} / \mathrm{cm}^{2}-\mathrm{sec}$.

${ }^{b}$ The numbers given with the metal screens refer to the thickness, in mils, of the front and back screens, respectively.

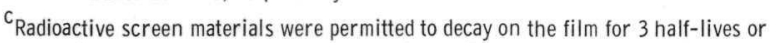
more after the neutron exposure was completed. For most of these materials (except indium) this extra transfer time produced little change in film exposure (see Ref. 1). ${ }^{d}$ The gadolinium screens available for this study have been very small (see Fig. 10) until recently, when $5 \times 7$-in. screens were received. These speed numbers should be regarded as tentative until further data can be obtained with these larger screens. The screen thicknesses given appear about optimum for speed, based on information from these initial tests. 
The double-screen method with cadmium screens and type KK film has arbitrarily been rated 1.0.(16) It should be emphasized that this speed information is strictly true only for the exposure conditions used here (2-min exposure for double screens and 4 min for single screens at a neutron intensity of $3 \times 10^{5}$ thermal n/ $\left.\mathrm{cm}^{2}-\mathrm{sec}\right)$. For other neutron intensities and exposure times, speed variations would occur as a result of failures of the reciprocitylaw with the scintillator and because of the different times involved for saturation activity with the radioactive screens. These speed values may be used as guides, however, over a rather broad range of exposure conditions. (17)

A few comments about each of these screen materials, including the reasons for the choice of the single-screen thickness and film placement shown in Table I, are given below. The investigations of image sharpness are still in progress, so that such information given in regard to these materials should be considered as preliminary.

Scintillators

The $\mathrm{B}^{10}$-loaded scintillator $(11,12)$ has yielded very fast detection results with type $\mathrm{F} X$-ray film. The sharpness of the radiographs obtained with this material has been very good and, in fact, among the direct-exposure methods it is second only to that obtained with gadolinium screens. The major drawback of this detection method is that the radiographs obtained with the scintillator have a mottled background which appears to be caused by nonuniform distribution of the $\mathrm{ZnS}(\mathrm{Ag})$ phosphor. A picture demonstrating this is shown in Fig. 2 .

The other scintillators tested have been combinations of LiF and $\mathrm{ZnS}(\mathrm{Ag})$. Tests have been made with the scintillators both in the form described by Stedman (13) ( $\mathrm{LiF}, \mathrm{ZnS}(\mathrm{Ag})$, and Lucite powder moulded into buttons) and in the form used by Shull(14) ( $\mathrm{LiF}$ and $\mathrm{ZnS}(\mathrm{Ag}$ ) pressed into a metal form). With natural LiF and type $F$ film, in both cases a relative speed rating of about 8 (on the same basis as the values of Table I) was found in these tests for the Stedman-type buttons and about 17 for the 4:1 $\mathrm{ZnS}(\mathrm{Ag})$ - LiF mixtures. (18) Comparable speed values werefound with Polaroid type $57 \mathrm{film}$ (3000 speed). Pictures produced by these scintillators were relatively grainy. In all cases, the scintillators have been used as back screens during the neutron exposures. On the basis of these preliminary data with the lithium scintillators, it seems reasonable to point out that, with $\mathrm{Li}^{6}$-enriched material, scintillators of the type described by Shull and either type F X-ray film or Polaroid type 57 film would yield the fastest response to thermal neutrons of any of the methods tested in this study thus far. 


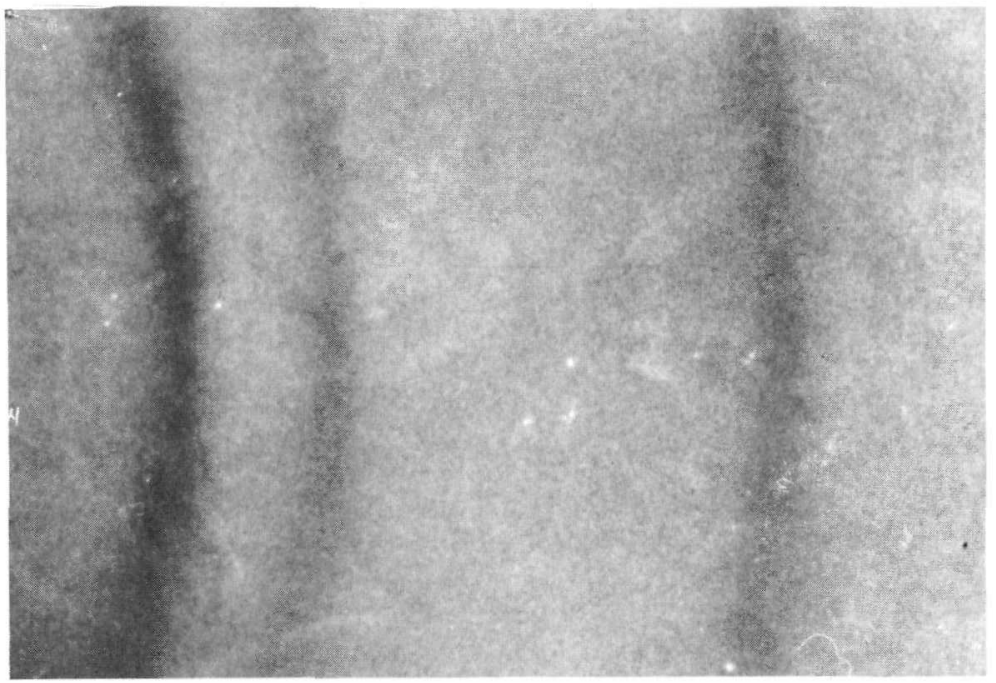

Fig. 2. A reproduction of a neutron-diffraction pattern obtained from a powdered nickel sample using a $\mathrm{B}^{10}$-loaded scintillator and type F X-ray film in a flat cassette. Typical exposure for such a picture is about $1 \mathrm{hr}$. The cassette was approximately $15 \mathrm{in.}$ from a relatively thick sample ( $7 / 16$ in. in dia.). The mottled picture background is due to the scintillator. The lines visible on this picture are the 111, 200, and 220 lines of nickel, respectively, starting from the left.

\section{Rhodium}

The use of rhodium screens has yielded radiographs having the third best sharpness (behind gadolinium and the scintillators) of the direct-exposure methods. For a single screen the best film density for a given neutron exposure occurs for a screen thickness of about 0.010 in., as shown in Fig. 3. As with most of the single-metal-screen methods, the best photographic film density is obtained for back films (films used on the side of the screen opposite to the neutron source), whereas best sharpness qualities are found for front films (photographic film on the same side as the neutron source). In the preliminary studies of image sharpness made thus far, little difference in image sharpness has been observed for radiographs made with rhodium screens which varied from 0.003 to $0.010 \mathrm{in.}$ Therefore, for rhodium, a 0.010-in. screen and a front film would be recommended because of the improved speed over the thinner screens and because of the improved sharpness for the front film. 


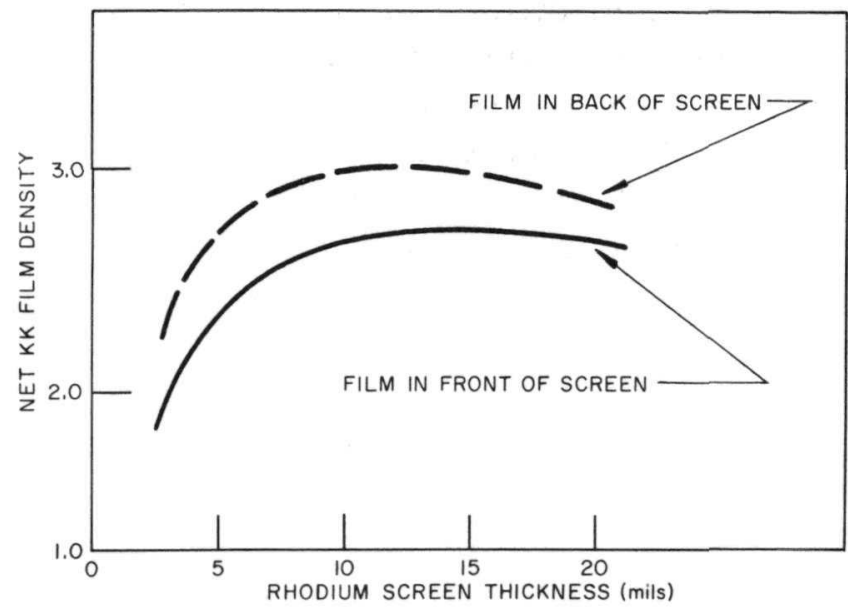

Fig. 3. Determination of single rhodium converter screen thickness and film location for best neutron photographic speed by the direct-exposure method. The information shown was obtained by exposing different screen thicknesses and film to the neutron beam for a constant neutron exposure, and comparing the resultant film densities produced. In the case of the radioactive screen-materials (shown in this figure and in Figs. 4 and 6) a three half-life decay period after the neutron exposure was given before the film and screen were separated. Films exposed on the neutron source side of the converter screen are called front films; those on the other side are called back films.

\section{$\underline{\text { Silver }}$}

The thickness of a single, silver converter screen which will yield the best film density for a given neutron exposure is approximately 0.015 in., as shown in Fig. 4. Little difference in image-sharpness qualities versus screen thickness has been observed for silver over the range from 0.005 to $0.020 \mathrm{in.}$. As in the case for rhodium, best film exposure was found for back films. For silver screens, however, the relatively low photographic speed, particularly for front films, may help account for the fact that little difference in sharpness was observed between front and back films.

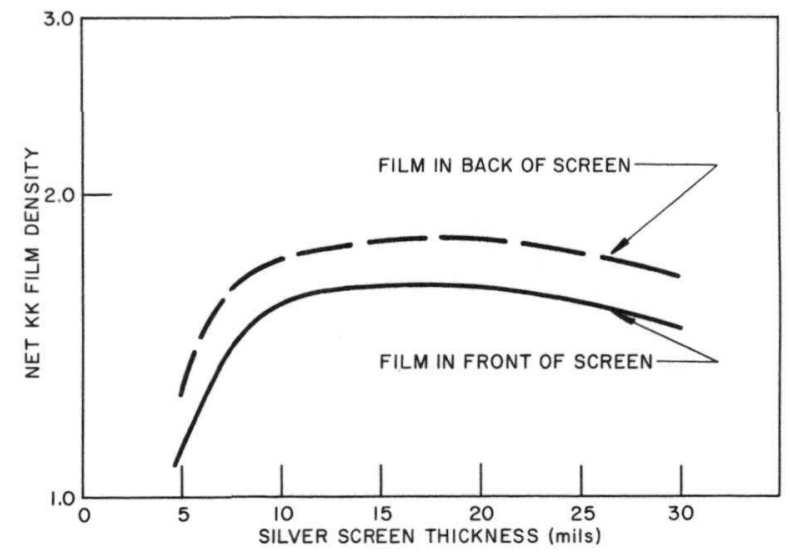

Fig. 4

Determination of single silver converter screen thickness and film location for best neutron photographic speed by the direct-exposure method. See caption on Fig. 3 for further details for this plot. 
It is thought that the increased influence of secondary and scattered radiation on the film during the long exposures needed for front films may have had some affect on this result. This was definitely true for the case of the prompt $(n, \gamma)$ radiation from the cadmium test object (see Fig. 10) used for the sharpness studies in that more film exposure from this radiation was found for front-film exposures than for back-film exposures. The choice for silver was therefore a 0.015-in. thickness screen and a back film.

\section{Cadmium}

The choice of the most useful cadmium screen and film placement was dictated by the facts that front films yielded much better sharpness than did back films and that this sharpness was relatively independent of cadmium thickness over the range from 0.001 to $0.010 \mathrm{in}$. The combination of good density and sharpness yielded by the 0.010-in. screen (see Fig. 5), and a front film was the choice for cadmium.

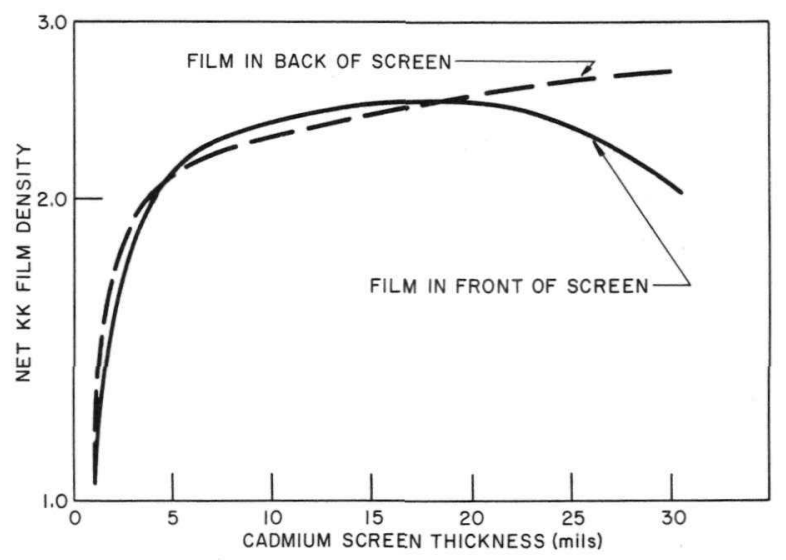

Fig. 5

Determination of cadmium converter screen thickness and film location for best neutron photographic speed by the direct-exposure method. See caption on Fig. 3 for further details for this plot.

\section{$\underline{\text { Indium }}$}

The sharpness found with indium screens appeared to be best for back films (perhaps for the reasons noted for silver exposures) and was relatively independent of screen thicknesses from 0.005 to $0.020 \mathrm{in.}$ Since a $0.020-i n$. indium screen and a back film also produced about the best film density, as shown by Fig. 6, this was the choice for direct exposures of indium.

Fig. 6

Determination of single indium converter screen thickness and film location for best neutron photographic speed by the direct-exposure method. See caption on Fig. 3 for further details for this plot.

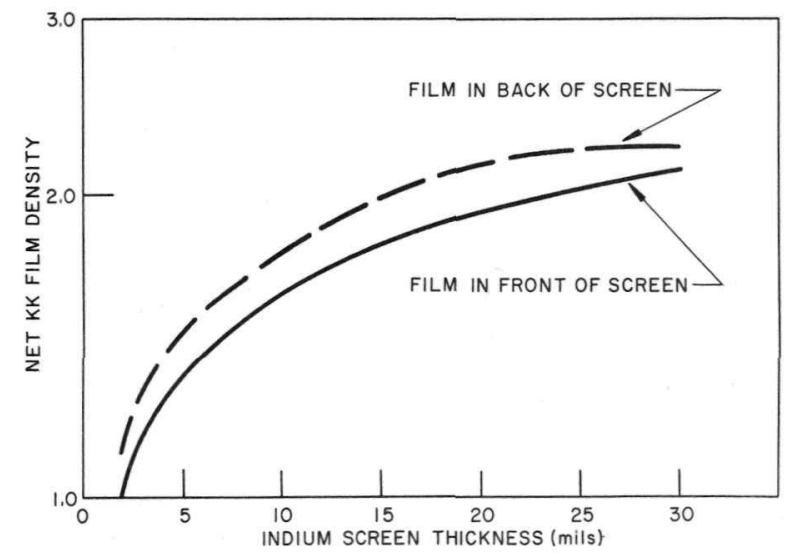




\section{Gadolinium}

Since useful size gadolinium screens have just recently been available for these tests, the gadolinium data must be regarded as preliminary. Without question, however, it can be stated that direct-exposure neutron radiographs taken with gadolinium screens have the best image sharpness of any of the other methods tested to date, including the transfer methods. Although a detailed study of image sharpness versus screen thickness has not yet been made, there is no obvious indication of sharpness change over the thickness range from 0.00025 to 0.002 in. for front films. In general, front films do yield appreciably better sharpness than back films. The thickness of gadolinium to use for best speed is of the order of 0.002 in. from the data shown in Fig. 7. This thickness of screen with a front film would be a highly recommended method for direct-exposure neutron radiography. It has good speed and excellent sharpness qualities.

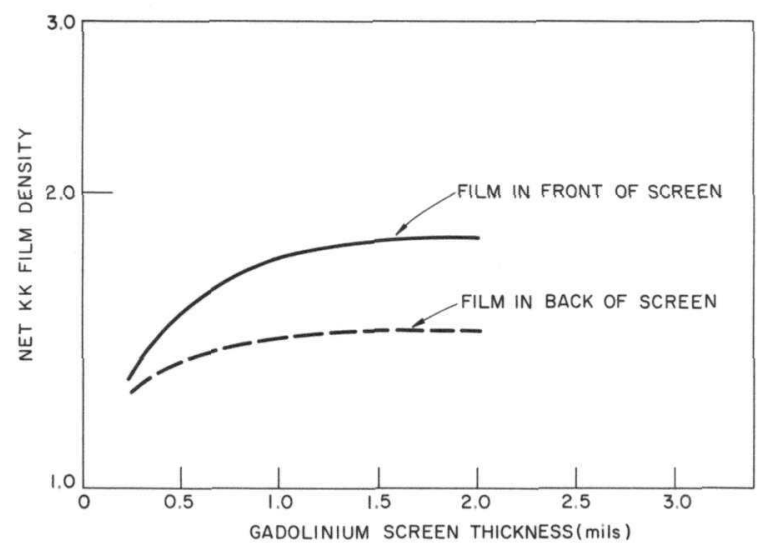

Fig. 7

Determination of single gadolinium converter screen thickness and film location for best neutron photographic speed by the directexposure method. See caption on Fig. 3 for further details for this plot.

Good image sharpness has also been obtained by the doublegadolinium-screen method. Almost equal speed results have been found for this method for both the 0.25-2 and 0.5-2 gadolinium double-screen combinations. As the front screen increases in thickness beyond 0.0005 in., the speed of the double-screen method begins to decrease. For the data shown in Table I, the speed of the 0.5-2 gadolinium combination was given.

Our present work with gadolinium involves a more detailed study of the image sharpness and contrast which can be obtained with the use of single gadolinium screens of different thicknesses, for double gadolinium screens, and for a single gadolinium screen used with a lead intensifier screen. (This last technique is further discussed in a following section of this report.) All these methods yield excellent image sharpness and it is hoped that, as a result of further study, a definite recommendation can be made for the best of these methods. 
Gold

Because of the low speed of direct-exposure methods of gold with double-screen techniques, (1) this method has not been fully tested for single-screen use. Gold, along with cadmium, produces direct-exposure neutron radiographs which have relatively poor image sharpness. This fact, along with the poor speed, would seem to render direct exposure of gold of limited use for neutron radiography, at least with neutron beam intensities of the same order as those used in these tests.

Other Direct-exposure Materials

Other materials which have been tested for direct-exposure neutron radiography include loaded emulsions and X-ray film itself. The speed results with both of these methods for neutron image detection were very low. (8) Speed numbers, on the same basis as those in Table I, were 0.03 for type KK X-ray film used alone(10) and less than 0.1 for both lithiumand boron-loaded emulsions (19) if density was taken as the basis of measurement.

The pictures obtained, particularly with $\mathrm{X}$-ray film, were normally of poor quality. The apparent reason for this is that, in the long exposures required, the film also recorded much other radiation, including that emitted from the objects under study and from the cassette, as well as recording gamma radiation in the imaging beam.

\section{B. Transfer Exposure Method}

Of the converter materials mentioned thus far in this report, only. indium and gold have yielded useful film blackening for the transfer method in the neutron intensity available. Rhodium and silver, which have reaction cross sections of the same order as those of indium and gold, have not been useful for transfer radiography with this neutron intensity because the short half-lives of the radioactive isotopes of these materials preclude their use with neutron exposures long enough to build up the required radioactivity. In addition to the fact that the influences of gamma radiation in the imaging beam and of radiation (other than neutrons) which may be emitted from the radiographic object are eliminated, transfer radiographs have the added advantage that they produce sharper pictures than direct exposures (with the noted exception of gadolinium).

Specifically, comparing indium and gold, much sharper radiographs can be obtained by the transfer method than by the direct-exposure method. Explanations for this result include the facts that some scatter is detected on the film during a direct exposure and that decreased contrast is obtained because the film is present to record prompt radiation emission from the 
absorbing object under study. A major cause for the change in sharpness, however, appears to be the fact that, in a direct exposure, the film is present to record relatively hard prompt $(n, \gamma)$ radiation from the converter screen itself. (8) In the transfer method, film darkening is accomplished by much softer radiation, thereby yielding improved image sharpness.

Of the two materials used for transfer radiographs, gold seems to yield somewhat better sharpness than indium. This result also may have its explanation in terms of radiation hardness. In each case, much of the film exposure would be caused by beta emission. However, there would also be an appreciable photographic effect yielded by the gamma radiation from radioactive decay. For In $^{116}$, a rather large portion of the total gamma emission occurs for gamma energies greater than $1 \mathrm{Mev}$. This is not the case for $\mathrm{Au}^{198}$, in that the energy of the most prominent gamma emission is $0.41 \mathrm{Mev}$. (20) This difference in gamma energy may explain the improved image sharpness found for gold transfer methods as compared with those using indium.

For the indium transfer method, Fig. 8 shows that the best speed is found for about a 0.020 -in. thickness, a thickness which is also the approximate range of the emitted beta energy. Figure 9 shows a similar result for gold transfers, with the best speed occurring for a thickness in the order of $0.005 \mathrm{in.}$ Both these curves are for front films. The exposure of film on the back side of the screen (not shown) begins to fall off at about the points where these curves level off, because of the combined effects of absorption of the neutrons within the screen material and the range of the emitted beta.

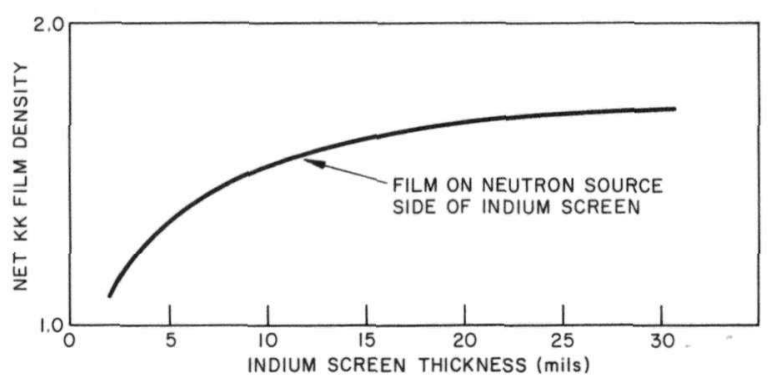

Fig. 8. Determination of single indium screen thickness for best neutron photographic speed using the transfer-exposure method. The information shown was obtained by exposing different thicknesses of indium foils to the neutron beam for a constant neutron exposure, transferring the foils to a film-loaded cassette, and comparing the film densities obtained after a 3 half-life transfer period.
The data on image sharpness for the transfer radiographs indicate little change in sharpness for transfers of gold in thicknesses of 0.003 and 0.005 in. The 0.005-in. screen would therefore be recommended because of its increased speed. The comparable data for indium show little detectable change in image sharpness for screens $0.010 \mathrm{in}$. or thinner (down to 0.002 in.). The 0.010-in. indium screen then appears to be a useful compromise between speed and image sharpness.

Further comparing these two materials, it should be mentioned that indium, because of its shorter 
half-life, does yield useful film blackening in less elapsed time than does gold, both in regard to neutron-exposure time and to transfer time. However, for similar reasons, indium cannot be used (in neutron intensities such as those used here) with slow, fine-grain film, such as type $M$, because a saturated activity is reached for a relatively short neutron exposure (about $3 \mathrm{hr}$ ). Gold, on the other hand, having a 2.7-day half-life, can be profitably exposed for the order of a week. Exposures of the order of $2 \frac{1}{2}$ days plus a week transfer time have yielded good film densities for gold transfers to type $M$ film.

Both these techniques then appear to be useful methods for neutron radiography. Typical exposure information is being given in another publication. (10)

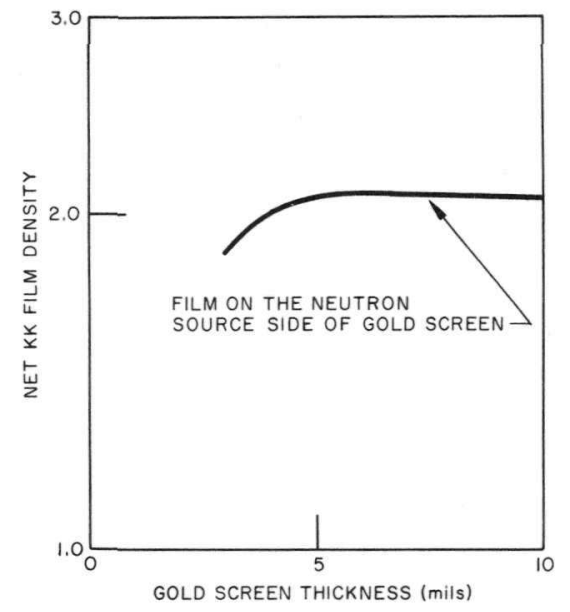

Fig. 9

Determination of single gold screen thickness for best neutron photographic speed using the transfer-exposure method. The information shown was obtained by exposing different thicknesses of gold foils to the neutron beam for a constant neutron exposure, transferring the foils to a film-loaded cassette, and comparing the film densities obtained after a 3 half-life transfer period.

\section{Other Neutron-imaging Observations}

In this section is briefly discussed some other observations. These include some preliminary trials with other neutron sources and some observations made with $\mathrm{X}$-radiographic intensification methods with neutron converter screen materials.

Our work with other neutron sources included the use of a 0.5-curie $\mathrm{Ra}-\alpha-\mathrm{Be}$ source and a Van de Graaff generator employing the $\mathrm{Li}^{7}(\mathrm{p}, \mathrm{n}) \mathrm{Be}^{7}$ reaction. The high gamma-to-neutron ratio output of the radioactive source has made direct-exposure methods with this source difficult, and the relatively low neutron yield has resulted in very light film exposures for trans fer methods, even using 3 half-life exposures and transfers for goldscreens to $\mathrm{KK}$ film. Although our results with this particular radioactive source have not been encouraging, it seems reasonable that usable neutron radiographs with higher yield sources (such as recently described by Hennelly(2l)) could be obtained. This possibility of using radioactive neutron sources for neutron radiography is further discussed by Watts. (6) 
The radiographic work with the Van de Graaff generator has been tried only with the $\mathrm{Li}^{7}(\mathrm{p}, \mathrm{n}) \mathrm{Be}^{7}$ reaction, employing a $2.5-\mathrm{Mev}, 10-\mu \mathrm{a}$ proton current and a thick lithium target. In these initial trials, the best, and aproximately equal, imaging results were obtained with 2 different methods. In one, the neutron beam was moderated only by the thick target and airpath, whereas in the second, the neutron beam was moderated by 2 in. of surrounding paraffin and the beam was brought out through a l-in.-diameter cylinder which did not "look at" the fast neutron source. The neutron intensity from each of these methods was such that, at 12 in. from the source, exposures approximately 10 times as long as those needed with the monochromatic neutron beam at the reactor were required. The effective neutron intensity at that distance was therefore of the order of $10^{4}$ thermal neutrons $/ \mathrm{cm}^{2}-\mathrm{sec}$. The gamma intensity under these conditions was relatively high, and it is estimated that 20 to $30 \%$ of the film exposure was the result of gamma radiation.

Although our results with the accelerator neutron source are also not too encouraging, it must be emphasized that, at this time, relatively little effort has been made here to use such other neutron sources for radiography. Further and more extensive investigations employing these other source type are presently in progress at other laboratories. $(23,24)$

When metal screens and films are exposed directly to the neutron beam to record the neutron image, a large portion of the photographic effect is produced by gamma radiation, both in the form of prompt $(n, \gamma)$ associated with neutron capture and the gamma radiation associated with the radioactive decay of the metal screen. Therefore, intensifying methods used in X radiography would seem applicable to these neutron-detecting methods. The influence of both lead screens and fluorescent screens on these radiographic detection methods for neutrons has been briefly investigated with the monochromatic thermal neutron source described earlier.

Par speed X-ray screens used with the metal converter materials have led to speed increases in the order of 25 to $50 \%$, with little obvious loss in image quality. These speed increases were found for single metal converter screens and a single fluorescent screen sandwiched around the film. These tests were made with types KK and AA X-ray films, and even better speed improvements might be expected with a film such as type $F$ film.

Best results using lead screens also were found by sandwiching the film between the single converter screen and the intensifier screen (lead in this case). For both lead and fluorescent screens less intensification was found by sandwiching the film between 2 extra intensifier screens, because then the screen between the converter and the film tended to absorb some of the softer radiation emitted from the converter screen. With the 
addition of a single 0.005 -in. lead screen, speed increases of the order of $50 \%$ over only the converter screen and film alone have been found for cadmium and gadolinium and of the order of 10 to $20 \%$ for rhodium, indium, and silver. Since much of the photographic effect contributed by cadmium and gadolinium screens is due to prompt $(n, \gamma)$ radiation, the larger speed increases with the use of a lead screen with these materials seems reasonable. There appears to be no significant loss in image quality with the use of the lead screen.

This last observation is now being further studied, particularly in regard to the use of a lead intensifying screen and a gadolinium converter screen. The relatively large increase in speed and excellent image sharpness of this combination is very attractive. The large increase in speed appears to occur because the soft radiation from the converter screen is still permitted to reach the film emulsion easily along with the added contribution of the secondary radiation from the lead screen caused by the harder radiation which would otherwise not have been detected by the film.

\section{$\underline{\text { Discussion }}$}

Gadolinium appears to possess all the qualities desired of a metal screen for use in direct-exposure neutron radiography. The tendency of this material in its naturally occurring form to become radioactive is negligible, meaning that additional transfer time after the neutron exposure is completed is not necessary and that there is very little, if any, possibility of "double exposures" by using the same screen for two consecutive radiographs. More importantly, the material has a very high cross section for thermal neutrons (46,000 barns), so that it can be used in small thicknesses with good speed. This is one factor involved in the excellent film sharpness that has been obtained with gadolinium. The second factor is that film blackening appears to be caused to a great extent by relatively soft radiation. (25) Measurements made here show this in the following manner. (8)

If 2 films are placed in front of a gadolinium screen in a directexposure neutron radiograph, the film farther from the screen will have about half the relative exposure of the film next to the gadolinium screen. By contrast with the other metal, direct-exposure screen materials, it is found that, for a similar test, the film farther from the screen will have 75 to more than $90 \%$ of the relative exposure of the film placed next to a rhodium, indium, silver, or cadmium screen. This increase in exposure for films placed a distance away from these other converter materials shows that more of the radiation emitted from these other screens is hard, penetrating radiation. It is true that this percentage relative exposure does vary somewhat with screen thickness because, with a thicker screen, more of the radiation is filtered by the screen itself before reaching the 
film, and is therefore harder. However, for most of the other screen materials, the use of thinner screens (and therefore somewhat softer radiation) becomes impractical because the increase in exposure required for those thin screens means that more scattered and secondary radiation will be recorded on the film, thereby decreasing the image quality. Even comparing similar screen thicknesses it is found that much more of the emitted radiation is absorbed in the film immediately adjacent to a gadolinium screen than for the other metal screen materials.

The soft radiation emitted from gadolinium and the high cross section for this material account for the fact that films placed on the neutronsource side of the gadolinium have greater relative exposure than back films (see Fig. 7). Most of the radiation emitted from the gadolinium appears to come from the first $0.00025 \mathrm{in}$. of thickness. As the screen becomes thicker, the soft radiation emitted toward the back film is filtered by the remaining screen thickness, so that both less relative exposure and poorer image sharpness for the back film are obtained as contrasted with the front film.

For the other metals, the muchlower cross sections for neutron reactions involved mean that the radiation emitted from the thicknesses used will be more or less equally emitted throughout the thickness of the material. Slightly more radiation will be emitted from the front surface and, what is more important from the sharpness point of view, the radiation emitted from the front surface will be stronger in softer radiation. The fact that greater density is found for back films rather than for front films with these other materials would seem to be the result of secondary radiation in the converter screen generated by the gamma emission of the screen itself. Metal intensifying screens used for gamma or X radiography are known to yield greater intensification for films placed behind the screen rather than for front films. (26)

An overall comparison of the qualities of image sharpness among the materials studied thus far rates gadolinium direct exposures, gold and indium transfers, the $\mathrm{B}^{10}$-loaded scintillator, and rhodium, indium, silver, gold, and cadmium direct exposures in the order of decreasing sharpness. A comparison of radiographs made with several of these materials is shown in Fig. 10. Eliminating the scintillator from the following discussion, and making use of data such as that mentioned earlier, in which several films were exposed together with a converter screen, it is generally found that good image sharpness is found for materials which emit softer radiation. This result is consistent with image unsharpness studies made with X radiation of various energies. (27)

A disturbing aspect of the data presented in this report is that little difference in image sharpness was found for rather large variations in converter-screen thickness. It is obvious for neutron radiography, as in the 
similar technique of autoradiography, (28) that as the screen, or sample, is reduced in thickness, the resultant radiograph will have greater contrast and better sharpness. These two qualities will both be improved because the radiation which reaches the film will originate from a thinner section, thereby giving the radiation less opportunity to spread before reaching the film.

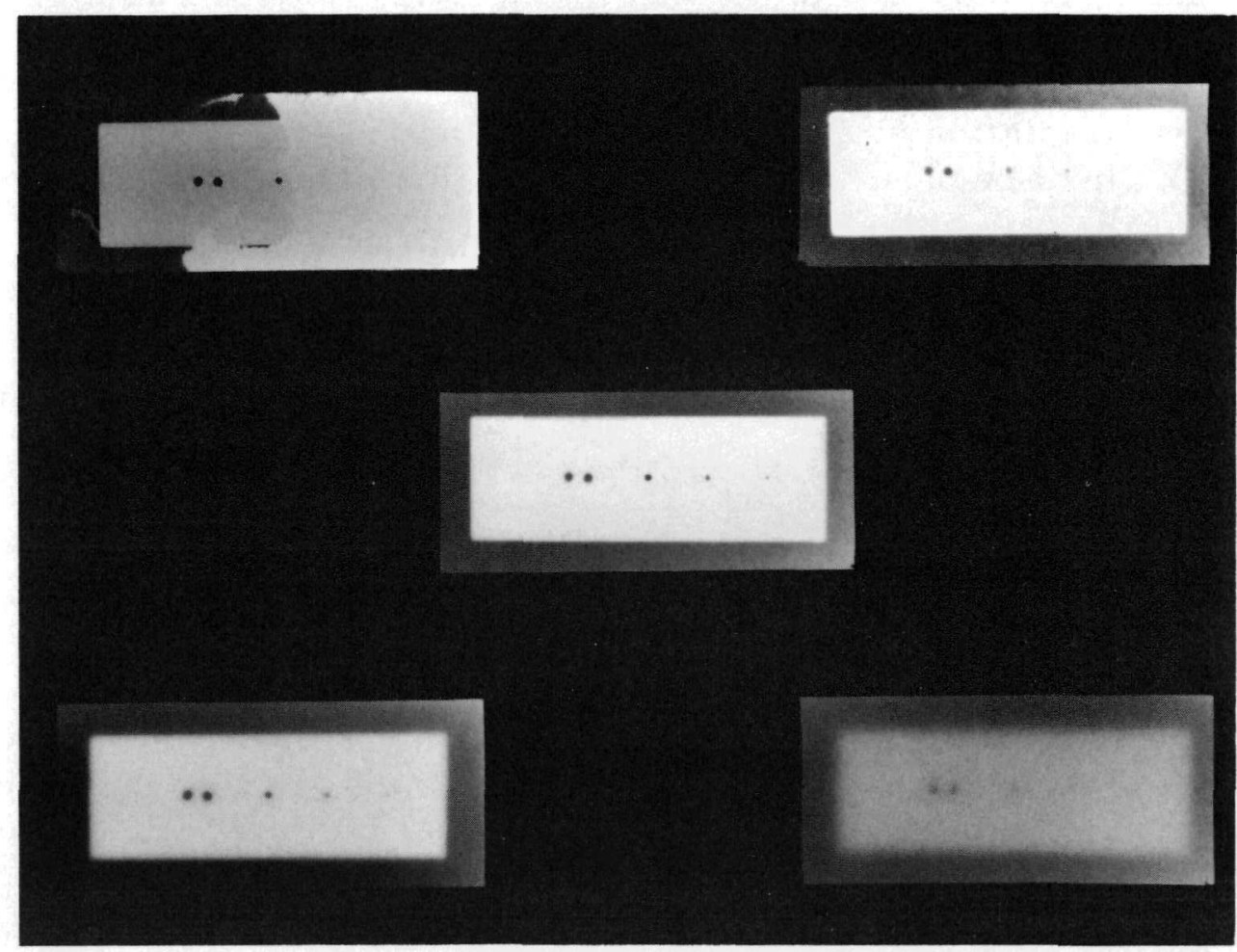

Fig. 10. A reproduction of several neutron radiographs of a cadmium test piece (0.100 in. thick) containing several drilled holes varying from 0.0135 to $0.040 \mathrm{in}$. in diameter. The radiographs were taken on AA film using a $0.00 \mathrm{l}$-in. gadolinium screen and a front film (odd shape is that of available gadolinium screen when radiograph was taken), top left; 0.005 in. gold in a transfer method, middle; double rhodium screens (10-10), bottom left; and double cadmium screens (10-20), bottom right. The $\mathrm{B}^{10}$-loaded scintillator is shown at the top right.

For the direct-exposure methods, the increased neutron-exposure times necessary for the thinner screens may have allowed more secondary and scattered radiation to influence the film, thereby also influencing the sharpness result. This explanation, however, does not seem entirely appropriate for the transfer radiographs. A possibility that does present itself is that it may be only by using much thinner screens for transfer radiography that significant improvements in image sharpness can be obtained. Other possible points to consider include our methods for maintaining good film-screen contact and for determining the image sharpness of the resultant radiograph. (8) Our techniques in both these areas are presently being re-evaluated. 
Good screen-film contact is, of course, a very important condition which must be maintained for best results. That this is one of the places in which improvement in our present methods appears necessary is shown by the transfer neutron radiograph of samples of boron carbide poison elements for a reactor, given in Fig. 11. The radiograph was taken by exposing the objects (29) and a 0.005-in. gold screen to the neutron beam for $2 \frac{1}{2}$ days, then transferring the radioactive gold to type $M$ film in a springloaded X-ray cassette for 8 days. Areas of poor film-screen contact on the radiograph are obvious. That these unsharp areas on the radiograph shown were the result of poor screen-film contact is more or less confirmed by the fact that the film exposed on the other side of the radioactive gold screen was essentially the reverse of the one used for Fig. 11, as far as image sharpness is concerned. Vacuum cassettes are presently being considered as a means of overcoming such difficulties.

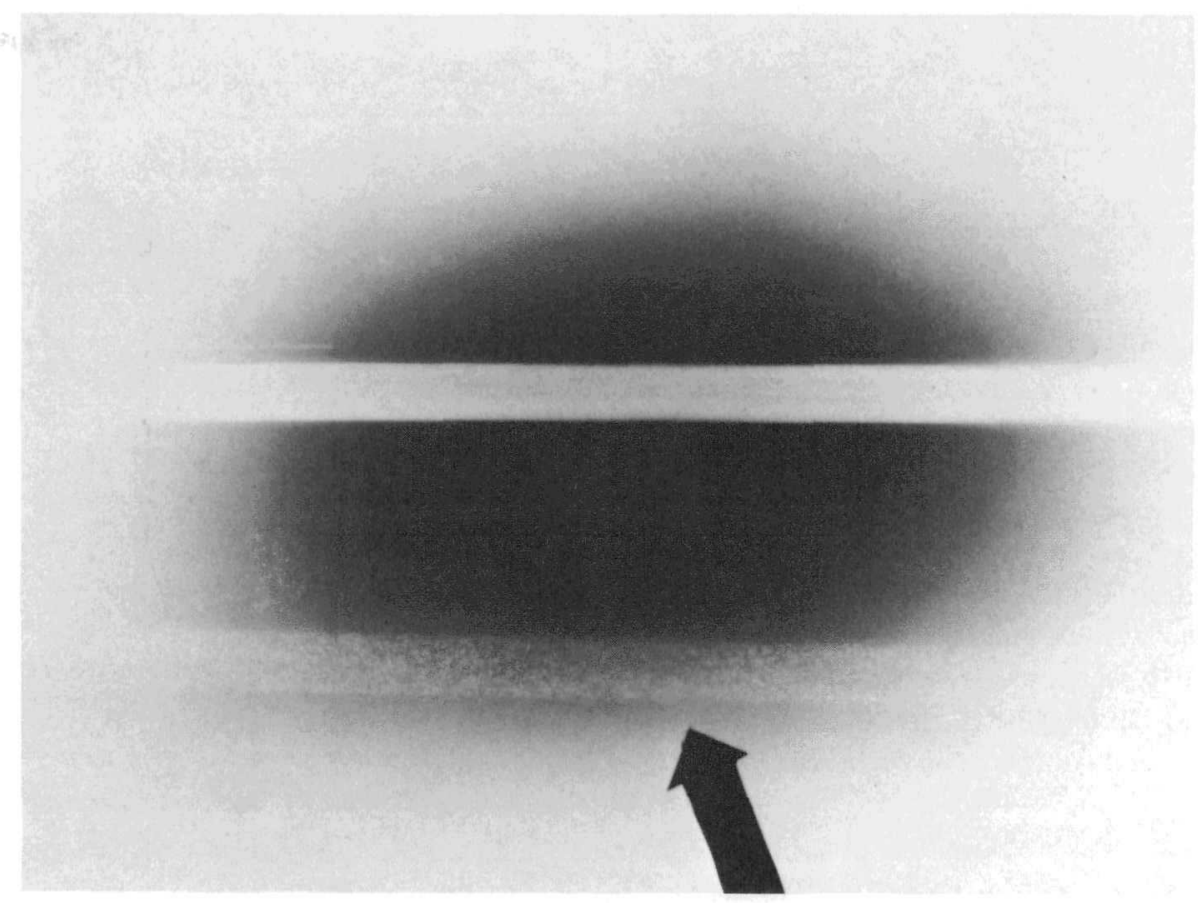

Fig. 11. A reproduction of a neutron radiograph of 2 rectangular cross-section, zirconium-clad, boron carbide reactor poison elements. This is a negative print, as are all the radiographs shown, so dark areas represent areas of high neutron intensity. The whitish spots within the shadow of the bar-shaped objects (particularly in the image of the thinner of the two samples, lower view) show the locations of the boron within the samples. The arrow points out a poor film-screen contact area which resulted in poor image sharpness. 
Since this discussion is in the nature of a progress report, it seems natural to make some comparisons between results which can be obtained now and those which were reported last year. From the point of view of the radiographs themselves, the most obvious improvement is in image sharpness. This is illustrated in Figs. 12 to 15, which show some comparisons of recent radiographs with those published in the first report. (1)

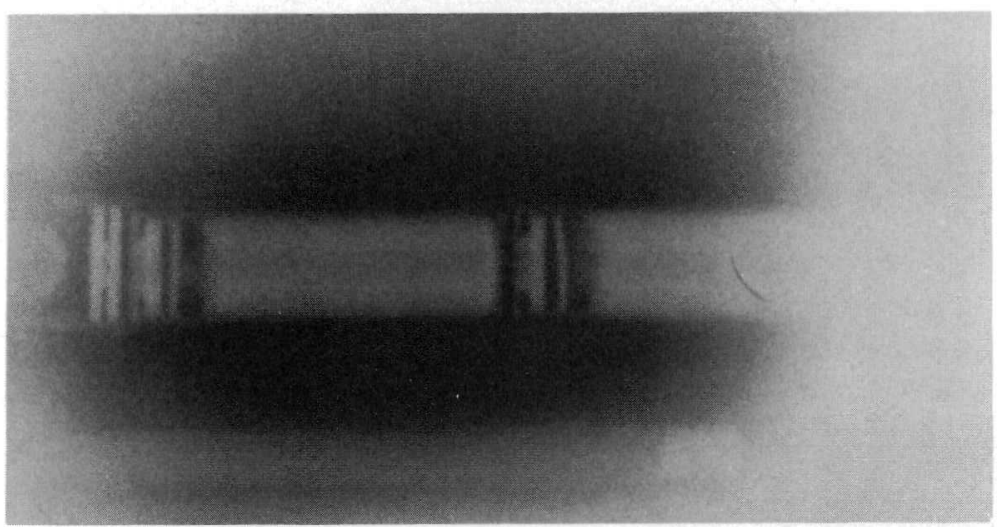

Fig. 12

A reproduction of a neutron radiograph of a pocket flashlight taken using a double silver screen directexposure method (18-18 screens) and No Screen film. This radiograph was taken in 1960 and was used as Fig. 6 of Ref. 1. The neutron exposure time was $6 \mathrm{~min}$.

Fig. 13

A reproduction of a neutron radiograph of the same object as shown in Fig. 12. This was taken recently by a direct-exposure method using a 0.002 -in. gadolinium screen and type $M$ film. The exposure time was $2-1 / 2 \mathrm{hr}$. These neutron radiographs look very different than $X$ radiographs of the same object, primarily because of the different absorption of the plastic components within the flashlight and the hydrogenous material in the batteries.
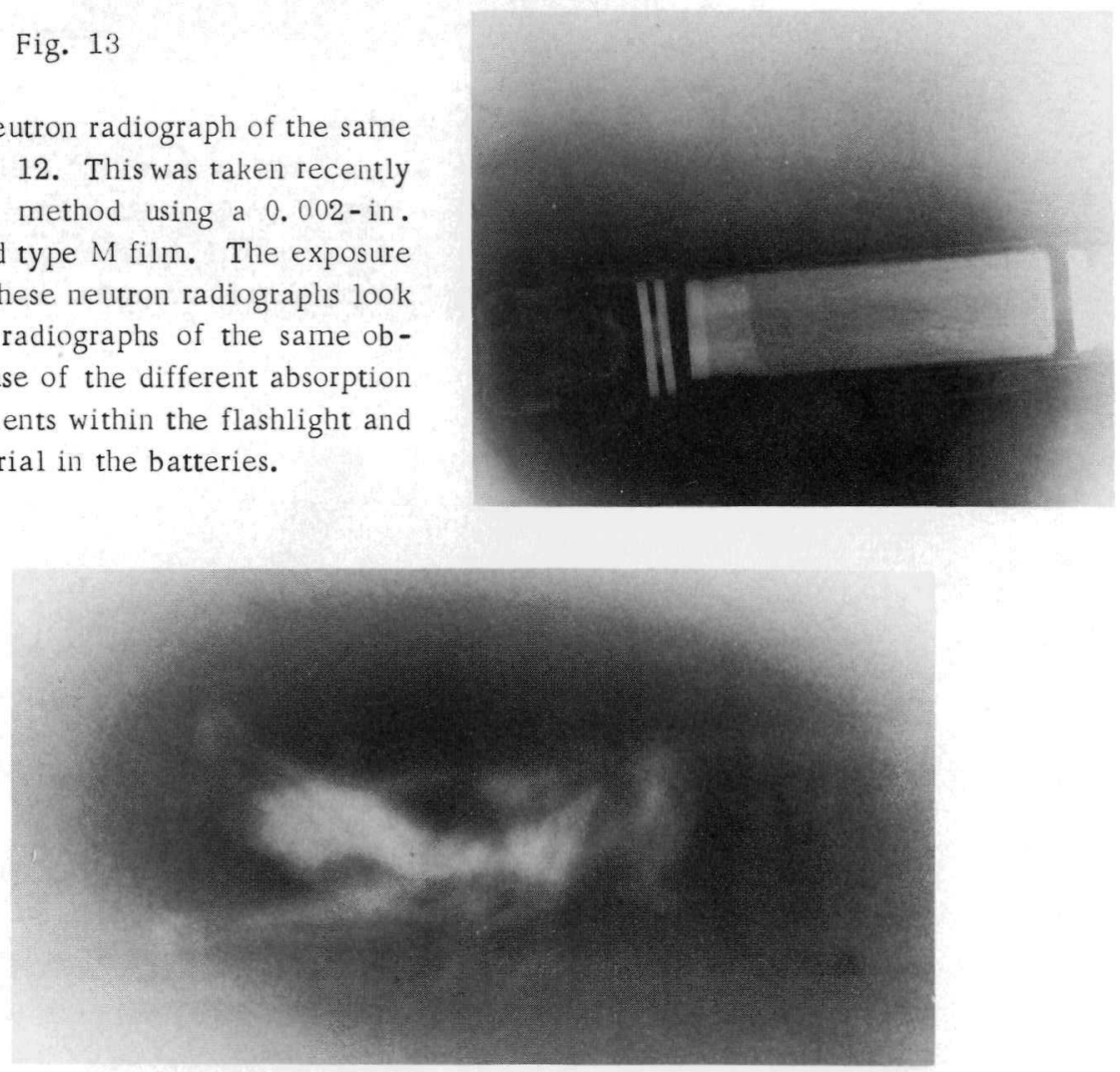

Fig. 14. A reproduction of a neutron radiograph of a grasshopper taken with double indium screens $(20-30)$ and AA film by a direct-exposure method. The neutron exposure was $40 \mathrm{~min}$. This radiograph was taken in 1960 and was used as Fig. 9 of Ref. 1. 


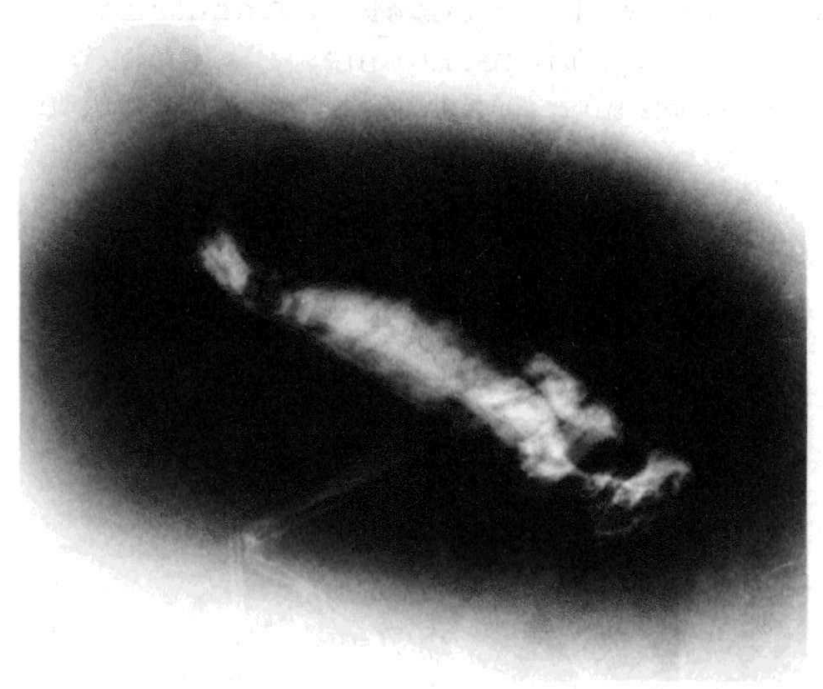

Fig. 15. A reproduction of a recent neutron radiograph of a grasshopper taken by the direct-exposure method using a 0.002-in. gadolinium screen and type $\mathrm{M}$ film. The neutron exposure was $2 \mathrm{hr}$.

This improved image quality now makes a discussion of possible areas of application for neutron radiography more attractive. Although a more detailed discussion of the possibilities of neutron radiographic application is planned for publication elsewhere, (10) some discussion of the objects pictured in the radiographs shown in this report does seem appropriate.

Figure 2 was shown primarily to demonstrate the mottled image background which is characteristic of the particular $\mathrm{B}^{10}$-loaded scintillator used in these tests. The lines on the picture are also of some importance in that they were caused by diffracted neutron beams from a powdered nickel sample. A film showing more lines from a similar sample (film was closer to the sample) is shown in Fig. 16. The use of film techniques for neutron diffraction work has not been uncommon for checking alignment and general set up.

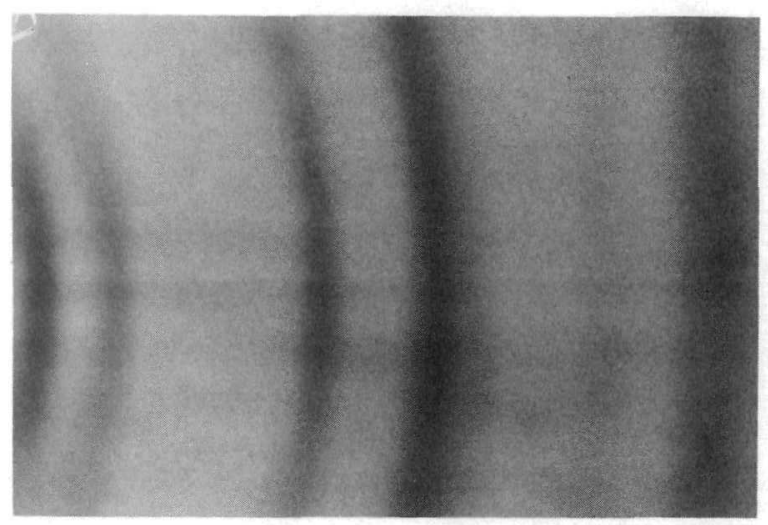

Fig. 16

A reproduction of a neutron-diffraction pattern of a powdered nickel sample taken with double rhodium screens $(10-10)$ and type $\mathrm{KK}$ film in a flat cassette. The cassette was approximately $6 \mathrm{in}$. from a relatively thick sample (7/16 in. in dia.) ). Exposure time for this picture was $15 \mathrm{hr}$. The diffracted lines shown begin with the 111 reflection on the extreme left. 
In addition, film has been used for recording neutron Laue patterns. (30) This extension of the use of film techniques for neutron-diffraction powder patterns may have some technical advantages in certain situations. (31) Although the use of film techniques for neutron diffraction may not be considered an application of neutron radiography, such use of neutronimaging methods may be an important byproduct.

Figure 11, in addition to demonstrating the importance of good film-screen contact, also easily shows the distribution of bor on within the inspection samples, a problem which would present difficulties with other test methods. Here, it illustrates the fact that absorption differences between neutrons and $\mathrm{X}$ rays can be used to advantage. The X-ray absorption of the materials within the object (zirconium and boron carbide) is approximately the same, whereas the neutron absorption is appreciably different, the neutron absorption of the boron being much higher than that for any of the other materials present. Such a study of the distribution of a highneutron-cross-section material within a mixture or alloy with other, lowneutron-cross-section materials, is a natural application for neutron radiography.

Other possibilities of application are discussed in the previously mentioned publication. (10) The reader is also referred to the more complete list of possible areas of application for neutron radiography given by Watts.(32)

The efforts of the past year have also led to some clarification of the requirements for good photographic, neutron-detection converter materials. For the direct-exposure method, gadolinium seems about perfect because of its very high cross section and because it emits relatively soft radiation. An additional requirement for a transfer-technique material would be convenient half-life for the radioactivity. Very recent tests with dysprosium screens indicate that this rare earth metal may satisfy these requirements. (33) Tests with both these promising materials are continuing.

\section{Acknowledgments}

The author would like to express his appreciation to Dr. W. J. McGonnagle for suggesting this research problem and for his cooperation during its investigation, to Mr. I. R. Kraska for his assistance throughout this study, to Dr. S. S. Sidhu and his neutron-diffraction coworkers for their helpful discussions and for their cooperation in making neutrons available, and to Dr. A. B. Smith and the Van de Graaff operators for their cooperation in the use of that equipment. 


\section{References}

1. For the first progress report see H. Berger, "Neutron Radiography," Symposium on Physics and Nondestructive Testing, Argonne National Laboratory, October, 1960, ANL-6346, pages 12-38.

2. Kallmann, H., Neutron Radiography, Research, 1, 254-260 (1947).

3. Peter, O., Neutronen-Durchleuchtung, Naturforsch, 1, 557-559 (1946).

4. Thewlis, J., Neutron Radiography, Brit. J. Appl. Phys., 7, 345-350 (1956).

5. Thewlis, J., "Neutron Radiography," Progress in Nondestructive Testing, 1, $111-126$, Heywood, Ltd., London (1958).

6. Watts, H. V., Investigations in Neutron Imaging, following paper, this symposium.

7. Ehrlich, M., The Sensitivity of Photographic Film to 3-Mev Neutrons and to Thermal Neutrons, Health Physics, 4, 113-128 (Dec 1960).

8. Berger, H., A Comparison of Several Methods for the Photographic Detection of Thermal Neutron Images, J. Appl. Phys., to be published.

9. Sidhu, S. S., L. Heaton, and M. H. Mueller, Neutron Diffraction Techniques and Their Applications to Some Problems in Physics, J. Appl. Phys., 30, 1323-40 (1959).

10. Berger, H., A Discussion of Neutron Radiography, to be presented at the 2 lst National Convention of Society of Nondestructive Testing, October, 1961, to be published, Nondestructive Testing.

11. The screen was a specially prepared version of the NE402 neutron detector commercially available from Nuclear Enterprises, Ltd., Winnipeg, Canada. A thin coating of silicone fluid and Saran film was applied on the light-emission side of the retangular scintillator. This thin protective coating allowed good contact between the scintillator and the photographic film. The active scintillator thickness was $0.3 \mathrm{~mm}$.

12. Sun, K. H., P. R. Malmberg, and F. A. Pecjak, High-efficiency Slowneutron Scintillation Counters, Nucleonics, 14 (7), 46-49 (July 1956).

13. Stedman, R., Scintillator for Thermal Neutrons Using $\mathrm{Li}^{6} \mathrm{~F}$ and $\mathrm{ZnS}(\mathrm{Ag})$, Rev. Sci. Instr., 31, 1156(Oct 1960). 
14. Shull, C. G., Massachusetts Institute of Technology, private communication. Professor Shull has modified the scintillator described by Stedman by using a mixture approximately 4 parts $\mathrm{ZnS}(\mathrm{Ag})$ to 1 part $\mathrm{Li}^{6} \mathrm{~F}$ by weight instead of the 2:1 mixture employed by Stedman. He also has eliminated the Lucite binder and has pressed the mixture into a metal dish. Professor Shull and his graduate students have designed a "neutron camera" employing this scintillator and Polaroid film. The camera appears to be a very useful device for checking the set-up of neutron-diffraction experiments.

15. The neutron beam used for most of this work was essentially parallel. Therefore no attempts have been made to place the object a distance ahead of the cassette in order to obtain geometrical magnification of the image.

16. The base of the table of speed values was changed from silver (Ref. 1) to cadmium because it was believed that cadmium, with little or no radioactivity involved, would be a useful base over a very wide range of exposure conditions.

17. As pointed out in Ref. 1, a large portion of the film darkening for a direct-exposure neutron radiograph occurs during and very shortly after the exposure. This is due to the combined effects of high cross sections for the short half-life activities and to the photographic effect of the prompt $(n, \gamma)$ radiation from the converter screen itself. These facts appear to account for the agreement in the literature on the order of speed for these metal screens over a wide range of conditions.

18. Argonne data tend to confirm the high speed of the 4-part $\mathrm{ZnS}(\mathrm{Ag})$ and 1-part LiF mixture used by Shull. Our results have shown a peak in speed for 4:1 and 5:1 mixtures by weight, when natural LiF was used.

19. Ilford Nuclear Research Plates, types K.2 (boron loaded) and K.1 (lithium loaded) were used in 50-micron thicknesses.

20. Strominger, D., J. M. Hollander, and G. T. Seaborg, Table of Isotopes, Revs. Modern Phys., 30, 585 (1958).

21. Hennelly, E. J., Intense Sb-Be Sources Make $10^{10}$ Neutrons/Sec, Nucleonics, $19(3), 124-5$ (Mar 1961).

22. With both the moderating configurations used, most of the neutron photographic effect appears to have been the result of resonance rather than thermal neutrons. This was confirmed by similar speed trial results obtained with or without a cadmium filter in the beam. The use of resonance neutrons resulted in improved relative speed results especially for silver, rhodium, and gold screens. 
23. Criscuolo, E. L., and D. Polansky, Progress Report on Neutron Radiography, Missiles and Rockets Symposium, U.S. Naval Ammunition Depot, Concord, California, April 1961.

24. Hirschfield, J., High Voltage Engineering Corporation, W. Leavitt, Watertown Arsenal, and M. Turkanis, Nuclear Materials Equipment Corporation, private communication.

25. Sala, O., P. Axel, and M. Goldhaber, Internal Conversion Electrons Accompanying Slow Neutron Capture in Gd, Phys. Rev., 74, 1249 (1948).

26. Seemann, H. E., Some Physical and Radiographic Properties of Metallic Intensifying Screens, J. Appl. Phys., 8, 836-845 (1937).

27. Halmshaw, R., and C. G. Pollitt, "Radiology with High Energy X Rays," Progress in Nondestructive Testing, 2, 3-26, The MacMillan Co., New York (1960).

28. Heller, D. A., J. A. Hamilton, "Radioautoradiography: Technic," Medical Physics, The Year Book Publishers, Chicago, 2, 817-823 (1950).

29. The objects were zirconium-clad, sintered, boron carbide bars, $0.225 \times 0.100 \mathrm{in}$. and $0.250 \times 0.225 \mathrm{in.}$ respectively, in cross section. The radiograph shows the distribution of boron within the bar. We are indebted to M. Turkanis of NUMEC for these reject samples, and to J. Ech of NUMEC and W. R. Plant of KAPL for their comments on interpretation of the neutron radiographs.

30. Wollan, E. A., C. G. Shull, and M. C. Marney, Laue Photography of Neutron Diffraction, Phys. Rev., 73, 527-8 (1948).

31. In the diffraction patterns shown, the lines are broad and diffuse. In the further efforts planned for this phase of the work, it is hoped that the use of samples of smaller size, better beam collimation, and curved film cassettes will yield improved results. The use of film to record neutron-diffraction patterns may be particularly advantageous for situations in which the weight or cost of an electronic detecting system may be prohibitive. There are also indications that film techniques may be capable of recording diffraction patterns in less time than that required for electronic detectors.

32. Watts, H. V., Research Study on Neutron Interactions in Matter as Related to Image Formation, Armour Research Foundation Report

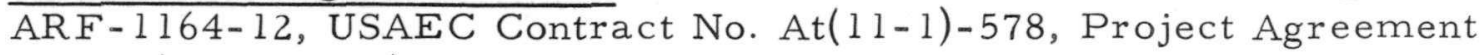
No. 2, (April 1961). 
33. Dysprosium-164 (28.1\% of naturally occurring material) has reaction cross sections of the order of 500 barns and 2000 barns, respectively, for the beta-emitting isotopes Dy-165m (1.25-min half-life) and Dy-165 (140-min half-life). See Ref. 20 and D. J. Hughes and R. B. Schwartz, Neutron Cross Sections, 2nd Edition, Brookhaven National Laboratory Report BNL-325 (July 1958).

Discussion

NEUTRON RADIOGRAPHY: A SECOND PROGRESS REPOR T

D. R. Green, General Electric, Hanford Atomic Products Operations: What is the lowest neutron energy available from your spectrometer facility?

H. Berger: The normal wavelength region in which the diffraction work is done is of the order of one angstrom, so that we have energies available of the order of $0.05 \mathrm{ev}$. Neutrons of lower energy are available from the spectrometer but, in general, as one moves either way from the energy distribution peak the beam intensities decrease.

G. M. Corney: Eastman Kodak Company: Were your gadolinium screens a layer of the pure element, or were they a salt ground up in a binder?

H. Berger: They were screens of pure gadolinium metal.

J. E. Bigelow, General Electric Company: Why do you use a monochromator at all? Is it just to get rid of the gammas, or is there another reason?

H. Berger: I used it mainly because it was the best neutron source I could find. I've been fortunate in that I have been able to tag on the end of the diffraction experiments and use the neutrons that they are essentially throwing away. I expect soon to get a beam facility of my own at one of the other reactors, where we will have a somewhat higher neutron intensity and where we will use the beam directly as it comes out of the reactor.

J. E. Bigelow: Would gammas be a problem then, and, if so, what technique would you use to get rid of the gammas?

H. Berger: Gamma radiation in that beam probably will be a problem. I expect to study primarily transfer techniques with that beam facility. Whether we can get rid of the gammas by means of filtering remains to be seen. 
INVESTIGATIONS IN NEUTRON IMAGING*

by

\author{
H. V. Watts \\ Physics Division \\ Armour Research Foundation \\ Chicago 16, Illinois
}

\begin{abstract}
The effects of neutron scattering in a radiographic object and the effects of the characteristics (sensitivity and resolution) of a neutron imaging detector on the image quality are considered for various applications of neutron radiography. In the thermal or slow-neutron energy region, in which the greatest differences in neutron cross sections among various elements exist, scattering constitutes a major por tion of the total cross section of most of the common elements. Thus, the scattering effect is important when one considers certain neutron radiography. Results of both neutron images and analogous optically synthesized images, using a "spot diagram" which is related to the neutron scattering, will be shown and compared for various thicknesses of the neutron scatterer. An evaluation of proposed imaging-detector systems, with different sensitivities and resolution, for specific requirements of neutron radiography have been performed on an optical bench with the image-synthesis technique. Various neutron detectors which appear applicable for image work are discussed.
\end{abstract}

\title{
I. Introduction
}

Neutrons penetrate matter in much the same way as do $\mathrm{X}$ and gamma rays (although the nuclear reactions caused by neutrons are unique) in that they follow an exponential absorption law and have a high penetrating power in most materials. The total neutron-absorption coefficient or cross section is comprised, in general, of a true absorption (capture) component and a scattering component, again similar to X-ray absorption. A major difference between neutron and $\mathrm{X}$-ray absorption is the very random way in which the neutron-absorption coefficients vary with the atomic number of the elements, especially when considering thermal or slow neutrons (in the energy range between 0.025 and $10.0 \mathrm{ev}$ ). This is in contrast with the regular

* This work was supported by the Office of Isotope Development, U.S.A.E.C. 
increase in mass-absorption coefficient with atomic number for X rays. These differences are illustrated in Fig. l.in which the total massabsorption coefficients of the elements for neutrons of $0.05-e v$ energy and for $130-k e v X$ rays are shown graphically.

The similarity in behavior of neutrons and $X$ rays suggests that neutrons could be used for inspection or radiographic work as are X rays; and the differences in their absorption characteristics with various elements suggest that neutrons could be used in many cases where $\mathrm{X}$ or gamma rays would fail to have contrast.

Successful neutron radiographs were obtained long ago by Kallman and Kuhn (1) with an ion beam from a small discharge tube impinging on a target as a source of fast neutrons. They moderated the se neutrons with water and used a film and fluorescent-intensifier screen detector. Peter (2) also published some results of neutron radiography using a more intense beam discharge tube than that of Kallman and Kuhn and an X-ray film sandwiched between 4-mil-thick silver screens for detector. More recently, Thewlis (3) published neutron radiographs obtained with $\mathrm{X}$-ray film and indium screens serving as a neutron converter, and a nuclear reactor as the neutron source. His radiographs "...compare fairly well in quality with many gamma ray radiographs...," and Thewlis illustrated some of the various possibilities of neutron radiography, such as biological studies, inspection of neutron-shielding material, inspection of heavy metals, and the use of neutrons for inspection of materials which cannot be distinguished by other means. The classic example of this latter case is the neutron radiograph of a piece of waxed string in a 2 -in. lead block.

There had been no further publications or reports of the application of neutron imaging or radiography since Thewlis until the current period when H. Berger (4) of Argonne National Laboratory, (ANL) and E. Criscuolo and D. Polansky(5) of Naval Ordnance Laboratory (NOL) independently presented progress reports of their work on neutron radiography. The cur rent research studies of ANL, NOL, and our own have been complementary in their scopes and there has been little direct duplication of experiments. At this date we know of no working application of neutron imaging in routine use or in research use.

The work presented here is a progress report on a study designed to optimize the characteristics of a neutron-sensitive imaging-detector system to be compatible with the neutron-image quality. We have considered the use of thermal or slow neutrons for which the cross-section differences among various elements are large, but for which scattering constitutes a major part of the total cross section for many of the elements. The effect of this scattering upon the image quality can be appreciable in certain instances. Also, the sensitivity and resolution of the imaging-detector system will limit the image quality. In studying these effects, we have developed a technique to simulate the neutron-intensity pattern at the image plane optically. 


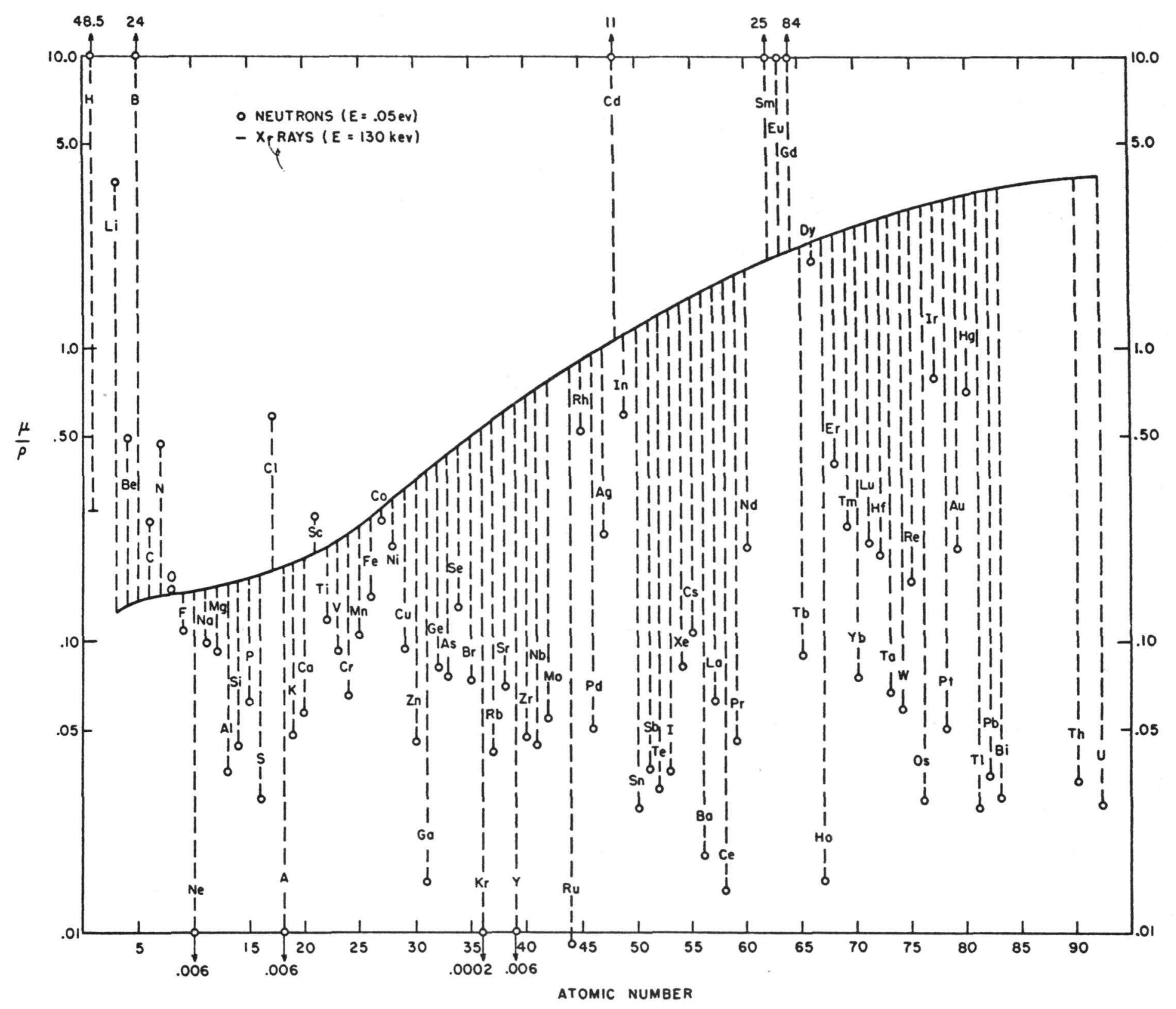

Fig. 1. Mass-Absorption Coefficients of the Elements for Neutrons and X rays 
This technique allows one to perform on an optical bench a parameter study of the neutron scattering and of the detector-resolution effects upon the image without fabricating costly neutron-imaging detector systems at this time. This is especially important in that we are concerned with evaluating possible new neutron-imaging detector systems, some of which do not exist today as complete operating units. We are considering the characteristics of neutron-image detector systems employing film techniques, multiwire spark chambers, arrays of small semiconductor detectors, luminescent materials (both in slab form and as bundles of fibers or rods), and opticalimage intensifiers or readout tubes in conjunction with luminescent materials.

\section{Applications of Neutron Imaging}

In general, the macroscopic neutron cross sections of the elements are such that greater thicknesses of material can be examined per unit of neutron flux than with the same intensity of $\mathrm{X}$ rays. Exceptions to this rule, as shown in Fig. 1, are at the thermal-neutron energies for which the elements of low atomic number, hydrogen, lithium, beryllium, boron, carbon, and nitrogen, the metal cadmium, and the rare earths samarium, europium, and gadolinium, have exceptionally high absorption coefficients. In addition, at thermal-neutron energies, there is a random and large variation in neutron cross section from element to element; this will permit many instances of discrimination not possible with X rays. For example, lead and gold have similar X-ray absorptions but dissimilar neutron absorptions. Conversely, tungsten and iron have very different $\mathrm{X}$-ray absorption coefficients but exhibit comparable neutron behavior. In this latter case, defects in a sandwich of iron in tungsten would be equally apparent for both constituents.

Examples of the differences between neutron and X-ray radiography are shown in Figs. 2a and 2b. The radiographic object in Fig. 2a is a $2-m m$-thick piece of iron on a tungsten wedge which tapers from zero to $2 \mathrm{~mm}$ in thickness. Slots are milled in the iron piece for identification, and the two pieces are held together with Lucite screws. The X-ray radiograph was taken at $165 \mathrm{KVP}$. Tungsten is quite opaque to $\mathrm{X}$ rays and, thus, the iron piece is visible through only the very thin section of tungsten; the Lucite screws, of course, are not visible at all with $\mathrm{X}$ rays. With thermal neutrons $(0.05-e v$ energy) the slots in the iron are visible for all thicknesses of tungsten, and the plastic screws are very pronounced. The slots in the screw heads show up very well. Figure $2 \mathrm{~b}$ is the radiograph of a pocket pencil dosimeter. The neutron radiograph shows the plastic structure which is hidden in the $\mathrm{X}$-ray radiograph. It is interesting to note that the wide opaque band that shows near the top of the neutron radiograph is a thin rubber gasket piece used to seal the metal cap of the dosimeter.

Because of these contrasts in absorption between various materials for thermal neutrons, the relative ease of producing moderated thermal 


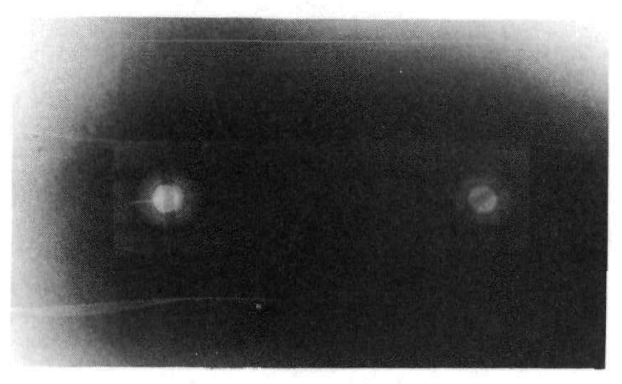

Neutron Radiograph

$\left(E_{n}=0.05 \mathrm{ev}\right)$

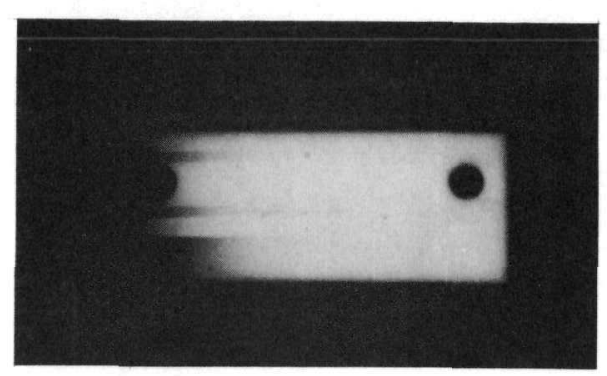

$X$-ray Radiograph

$\left(\mathrm{E}_{\mathrm{X}}=165 \mathrm{KVP}\right.$. $)$

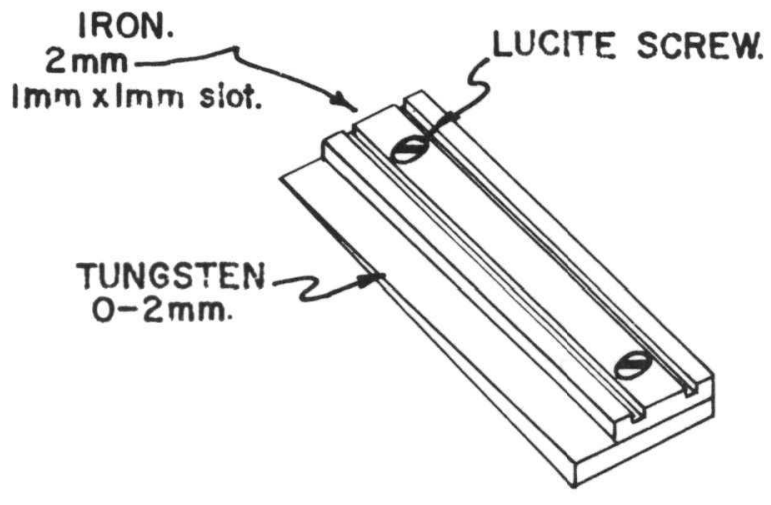

Radiographic Object

Fig. 2a. Neutron and X-ray Radiographs of Tungsten-Iron Absorption Object

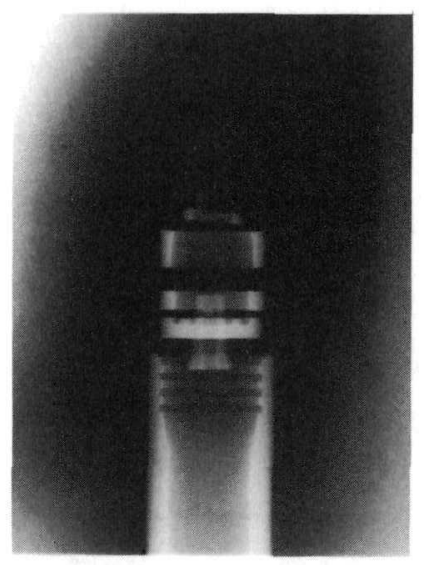

Neutron Radiograph

$\left(E_{n}=0.05 \mathrm{ev}\right)$

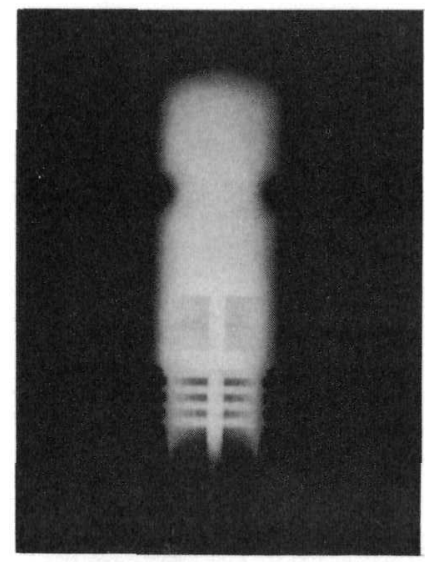

$X$-ray Radiograph

( $E_{X}=17$ KVP. )

Fig. 2b. Neutron and X-ray Radiographs of Pencil Dosimeter. 
neutrons from neutron sources, and the fact that thermal-neutron detection is more efficient than detection of neutrons of higher energy, it is likely that most neutron imaging will be performed with thermal-neutron sources.

However, there are possible advantages foreseen in using neutrons in the energy range of a resonance. In particular, if there is not a great contrast in absorption between an object and its surroundings in the thermalenergy region, then one can probably find a large contrast by going to the resonance-energy region, either of the object or of the surroundings. In some cases, the use of a resonance-energy region would be helpful in reducing the effect of scattering. These advantages can only be realized if appropriate sources of neutrons can be devised having the required energies and if good neutron detectors at these selected energies are available.

In the fast-neutron energy range there is little contrast between the cross sections of the elements, and the values of the cross sections are relatively low (about 1 to 4 barns). The extremely penetrating character of fast neutrons may prove advantageous in some cases. However, the scattering of fast neutrons is anisotropic and inelastic; the se effects on the image detection have not been evaluated.

Neutron imaging should provide a very valuable complement to $\mathrm{X}$-ray and gamma-ray techniques by virtue of the different response characteristics. Examples of applications of neutron imaging which illustrate the advantages of neutron techniques and the differences from conventional methods include:

Reactor Technology

1. Inspection of control rods or plates for homogeneity, continuity, and content of poison element.

2. Inspection of neutron shielding material for voids.

3. Location of organic coolants and organic residues in metal tubes or lines.

4. Inspection of thick fuel elements.

5. Study of inclusions in metals, such as hydrides or boron in zirconium.

Rocket and Missile Technology

6. Examination of large thicknesses of cast solid fuels for cracks or fissures.

7. Inspection of plastic or rubber tubing components in air frames.

8. Inspection of rubber or organic gaskets for correct position and seating in assembled metal units. 
General

9. Study of thin biological specimens, such as plant tissues, animal tissues, and insects, with much greater contrast.

10. Inspection of plastic materials, such as electrical insulation, for flaws and defects.

11. Inspection of cadmium plating for homogeneity and continuity.

12. Diffusion of materials, such as boron, in silicon or germanium.

13. Inspection of indium metal gaskets in pressure or vacuum systems.

The list is by no means exhaustive and, historically, once such a method becomes available, the number of uses multiplies many fold as the ingenuity of researchers is focussed on its application.

\section{Neutron Sources}

A nuclear reactor is a most prolific source of neutrons, but a rather impractical source in regards to its cost, size, and lack of mobility for many routine possibilities for inspection by neutrons. More pertinent to the future of neutron imaging are neutron sources of the accelerator type and isotopic neutron sources. A tabular survey of accelerator neutron sources, from the small ion beam tube type through the linear electron accelerator type, has recently been published.(6) In general, one can realize a neutron yield of the order $10^{10} \mathrm{n} / \mathrm{sec}$ from a neutron tube or generator priced at about $\$ 20,000$. The useful beamed neutron intensity from such a source would probably be of the order of $10^{6} \mathrm{n} / \mathrm{cm}^{2}-\mathrm{sec}$.

Conventional isotopic neutron sources, such as $\mathrm{Ra}-\mathrm{Be}, \mathrm{Po}-\mathrm{Be}$, and $\mathrm{Pu}-\mathrm{Be}$, are fabricated for neutron yields of the order of $10^{8} \mathrm{n} / \mathrm{sec}$. Recently, Hennelly $(7)$ has reported on a $\mathrm{Sb}-\mathrm{Be}$ photoneutron source which uses $10^{3}$ curies of $\mathrm{Sb}^{124}$ and produces $10^{10} \mathrm{n} / \mathrm{sec}$. This source has a half-life of 60 days and it has the gamma-ray background of the $2-\mathrm{Mev} \mathrm{Sb}^{124}$ gamma ray. Promising isotopic neutron sources appear from the production of the transplutonium elements. Americium-24l has been used to prepare an $(\alpha, n)$ source with beryllium.(8) A neutron emission of $6.29 \times 10^{6} \mathrm{n} / \mathrm{sec}$ from 3.38 curies of americium was obtained. The advantage of americium over plutonium is its greater specific activity, $0.32 \mathrm{gm} / \mathrm{curie}$ vs. $16 \mathrm{gm} / \mathrm{curie}$, and ame ricium has a much longer half-life, 470 years, than polonium or antimony. Also, even though americium emits a $60-\mathrm{kev}$ gamma ray, it can be used as a gamma-ray-free neutron source with moderate shielding, as compared with radium and antimony sources. Americium has not been released for use in neutron sources as yet. 
Looking further into the future of isotopic neutron sources, one can consider the transplutonium elements of higher atomic number which fission. spontaneously. It has been quoted(9) that $30 \mathrm{mg}$ of $\mathrm{Cf}^{252}$ will provide $10^{11} \mathrm{n} / \mathrm{sec}$ by spontaneous fission with a half-life of $2.2 \mathrm{yr}$.

Since neutron radiographs are readily obtainable on $\mathrm{X}$-ray film with activation foil converters with exposure times of minutes for neutron beam intensities of the order of $10^{6} \mathrm{n} / \mathrm{cm}^{2} \mathrm{sec}$, a neutron source with a yield of $10^{10} \mathrm{n} / \mathrm{sec}$ is adequate for use. Thus, today, there are small accelerator neutron sources available competitive with $\mathrm{X}$-ray installations, when one considers the potential of the inspection use. The future applicability of isotopic neutron sources, by virtue of improved neutron-image detection and/or higher yield isotopic neutron sources, for neutron radiography appears promising.

\section{Neutron-image Detectors}

Basically, all nuclear detection requires the interaction of an energetic charged particle with matter. The detection of a neutron then requires an additional interaction which results in a charged particle related to the neutron. Neutron conversion interactions can be grouped in three broad categories $(10)$ :

1. Neutron-induced transmutations in which the product particles or gamma rays make possible the detection, such as the $(n, \alpha)$, $(n, p),(n$, fission), and $(n, \gamma)$ reactions. The alpha particle, the proton, the fission product, or the gamma ray give instantaneous information concerning the neutron.

2. Neutron-induced transmutations which result in a radioactive product nucleus. The subsequent decay particles or radiation from the radioactive nuclei give information concerning the neutron. We will call the se activation converters.

3. Elastic scattering of neutrons in which the recoil particle is charged and can be detected. The scattering of a neutron by a proton is the most important example of this process.

Boron-10 and lithium-6, both having high $(n, \alpha)$ cross sections for thermal neutrons, are the most commonly used neutron converters in category 1. When these materials can be used as a homogenously mixed additive in a detector, such as a scintillator, neutron-conversion efficiencies of 50 percent and greater are obtained within reasonable thicknesses. When these neutron-converter materials are used in a layer form, where it is the surface of the layer to be detected, the conversion efficiency is restricted to the order of one to five percent because of the short range of the alpha particles $\left(1-3 \mathrm{mg} / \mathrm{cm}^{2}\right)$. Where possible, one could consider using 2 converter layers, one on each side of the charged particle detector. 
The characteristics of several activation-converter materials (category 2) are given by Berger.(11) Since the beta particles emitted from these materials have maximum ranges of the order of hundreds of $\mathrm{mg} / \mathrm{cm}^{2}$, converter foils of these materials may be used in this thickness range, and the direct neutron-conversion efficiencies can become reasonably high (>20\%). The activation materials with half-lives of the order of an hour are especially useful when there is a significant gamma-ray intensity present in the neutron beam and the primary detector is gamma-ray sensitive. In this situation, the activation foil may be exposed to the neutron image separately (it will not be affected by the gamma rays) and then transferred to a suitable betaparticle imaging detector to record the neutron image. This technique, called the activation-transfer method, is not, of course, as efficient as the direct activation method.

A proton-recoil conversion (category 3) in hydrogenous materials is limited in use to neutrons of energy greater than $0.1 \mathrm{Mev}$. In layer converters (a polyethylene sheet, for example), the conversion efficiency is restricted by the range of the protons. This efficiency is of the order of $0.1 \%$ for $4-\mathrm{Mev}$ neutrons. (10) Conversion efficiencies of several percent for fast neutrons are possible when the hydrogenous material is mixed homogeneously with a scintillator, such as Hornyak's(12) mixture of Lucite and $\mathrm{Zn} \mathrm{S}(\mathrm{Ag})$ and used in thicknesses of about one $\mathrm{cm}$.

If it is desired to discriminate strongly against neutrons below a certain value of energy, it is possible to use a neutron-induced nuclear reaction for which the cross section for the reaction is zero below a certain threshold energy and rises rapidly above that threshold value. The main requirement for use of these reactions is that the product of the reaction be a radioactive beta emitter. Considerations of this type of converter are essentially the same as for activation converters. Some of the threshold converters which can be used are listed by Cohen(13) and by Cowan and O'Brien. (14)

Detection Systems Applicable to Neutron Imaging

\section{Film Techniques}

In general, film techniques will have a resolution dependent upon the range of the charge particle from the neutron-conversion process used, or, if a slab of neutron scintillator is used with a film, the resolution will be dependent upon the thickness of the scintillator slab (assuming that the thickness of the scintillator slab is larger than the range of the charged particle in the scintillator). Films are also sensitive to gamma rays and thus, if the neutron beam has an appreciable gamma-ray intensity mixed with it, one would have to use the activation-transfer method. Data on the relative photographic speeds and resolution for film techniques utilizing various neutron converters have been reported by Berger.(1l) 


\section{Multiple Wire Spark Counter}

The feasibility of using a novel type of multiwire spark counter as an imaging detector for thermal neutrons was demonstrated several years ago by Reiffel.(15) The highly localized development of luminous spark channels in the region of a suitable initiating event forms the basis for the method. The electrode configuration of Fig. 3, operating in air

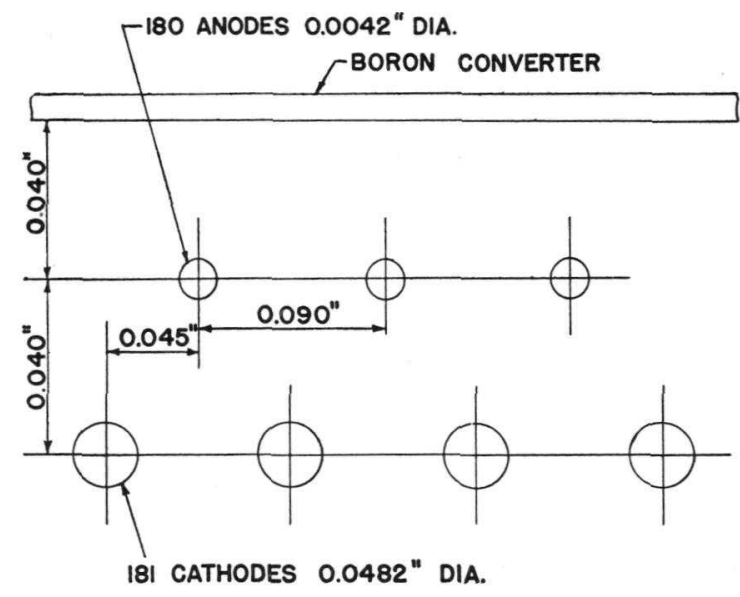

Fig. 3. Electrode Configuration of Multiwire Spark Counter. or in an atmosphere of 10 percent methane and 90 percent argon, can be triggered by highly ionizing events, such as alpha-particle tracks. The $(n, \alpha)$ neutron converter used is an enriched boron-10 layer which sits at a distance of 0.040 in. from the center of the plane of the anode wires. The counter operates at about 2000 volts in a methane-argon atmosphere and at about 4000 volts in air at atmospheric pressure. The device is operated as a flow counter from a gas supply of known quality to insure controllable and reproducible characteristics.

The electrode configuration of Fig. 3 is considerably less directional than the more usual wire-andplate or on-center-wire configuration, and exhibits close to 100 percent efficiency for counting alphas. With the enriched boron-lo converter layer one obtains an overall efficiency for the detection of thermal neutrons of about 5 percent. The counter can be operated in very high gamma-ray fields (up to $10^{5} \mathrm{r} / \mathrm{hr}$ ) without causing any increase in the background counting rate, which is of the order of counts per hour. This property is of great importance in reactor experiments.

Each spark discharge, representing the detection of a single alpha particle, which in turn is produced by one detected neutron, can be photographically recorded and contributes one element to building a picture of the incident radiation distribution over the area covered by the electrodes. Since the individual sparks can be made almost arbitrarily bright, the factor of energy intensification is extremely large. The spark distribution can be recorded by a camera arranged to take a time exposure of the entire counter area. At low F-number settings of the camera lens, each spark is of sufficient intensity to saturate a fast optical film, and a "half-tone" picture of the incident radiation distribution results. At high F-numbers, a number of sparks are required to saturate a given area of the film, and an image with an extended gray scale is obtained. 
An experimental setup of the large-area (about $2000 \mathrm{sq} \mathrm{cm}$ ) spark counter at the ARF Reactor is shown in Fig. 4. Mylar sheets sealed to the front and back of the counter frame form the atmosphere control chamber for the counter. In this instance a portrait lens was used on the camera to obtain a one-to-one imaging of the spark counter wires on the film. A prism on the camera lens is used so that the camera could be set out of the neutron beam and still view the plane of the wires. At a camera lens setting of F 11 with Royal-X-Pan film, a number of sparks are required to saturate the film and thus a gray scale is produced. A thermal neutron beam intensity of about $5 \times 10^{5} \mathrm{n} / \mathrm{cm}^{2}-\mathrm{sec}$ is readily detectable in an exposure time of several seconds.

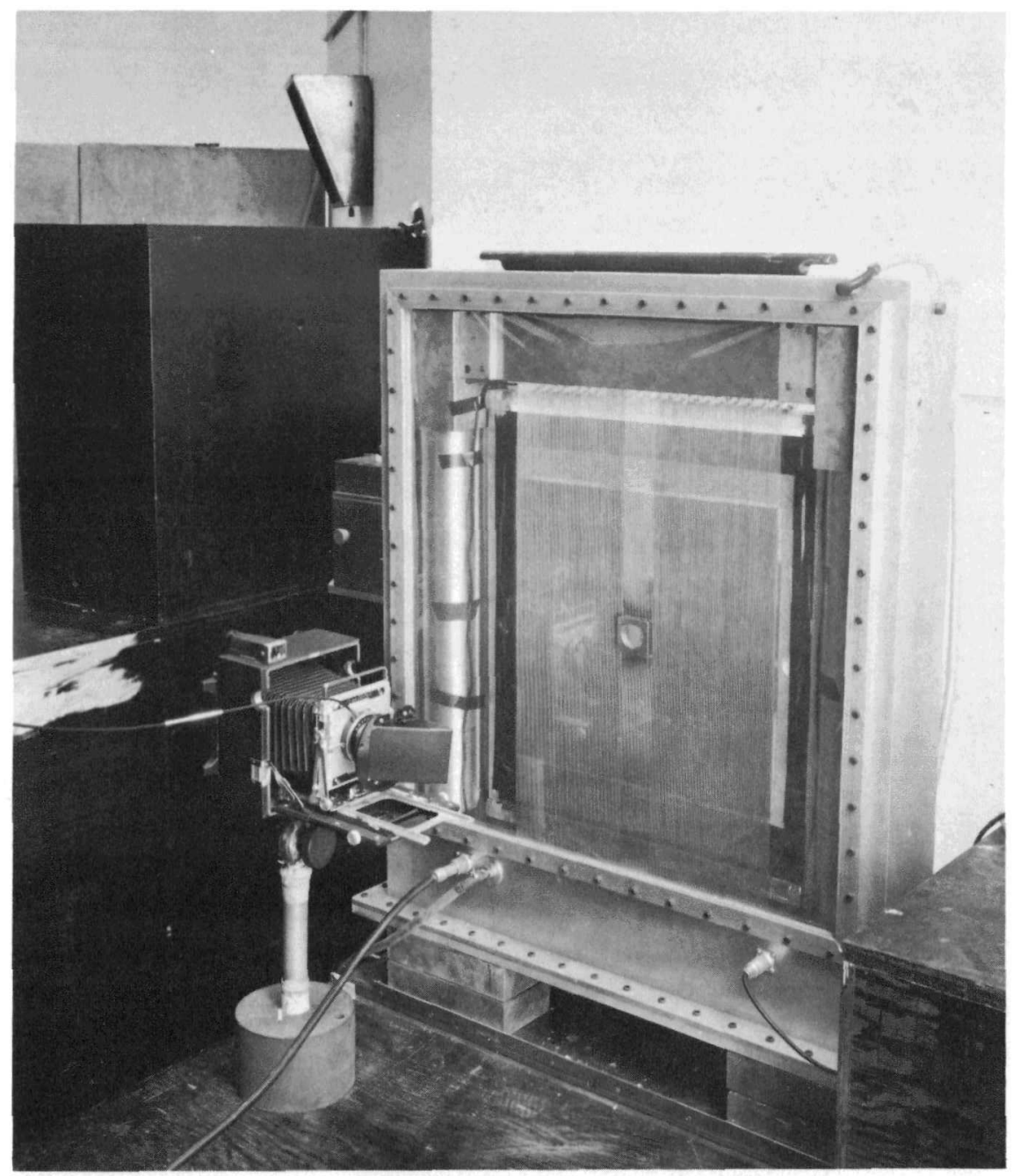

Fig. 4. Multiwire Spark Counter

The spark-counter image of a circular neutron beam of $6-\mathrm{mm}$ diameter is shown in Fig. 5. The spacing between wires is $2.3 \mathrm{~mm}$, and in this photograph the cathode wires have been superimposed on the spark 
pattern. The spark counter gives an elliptical pattern caused by the dependence of counting efficiency on alpha-track angle. Although, as stated earlier, this angular dependence is not as pronounced as in other spark geometries, it is still measurable. The counter maintains high efficiency to more grazing angles of incidence when the alpha track lies in a plane perpendicular to the electrode axis. This is because the radial electric fields of the counting geometry can collect charge from a track in the perpendicular plane and concentrate it into a very small volume, whereas for a track parallel to the electrodes, the radial fields cannot so concentrate the charge. Also, it is to be noted that collimator leakage or a penumbra effect around the main beam is discernible in this photograph.

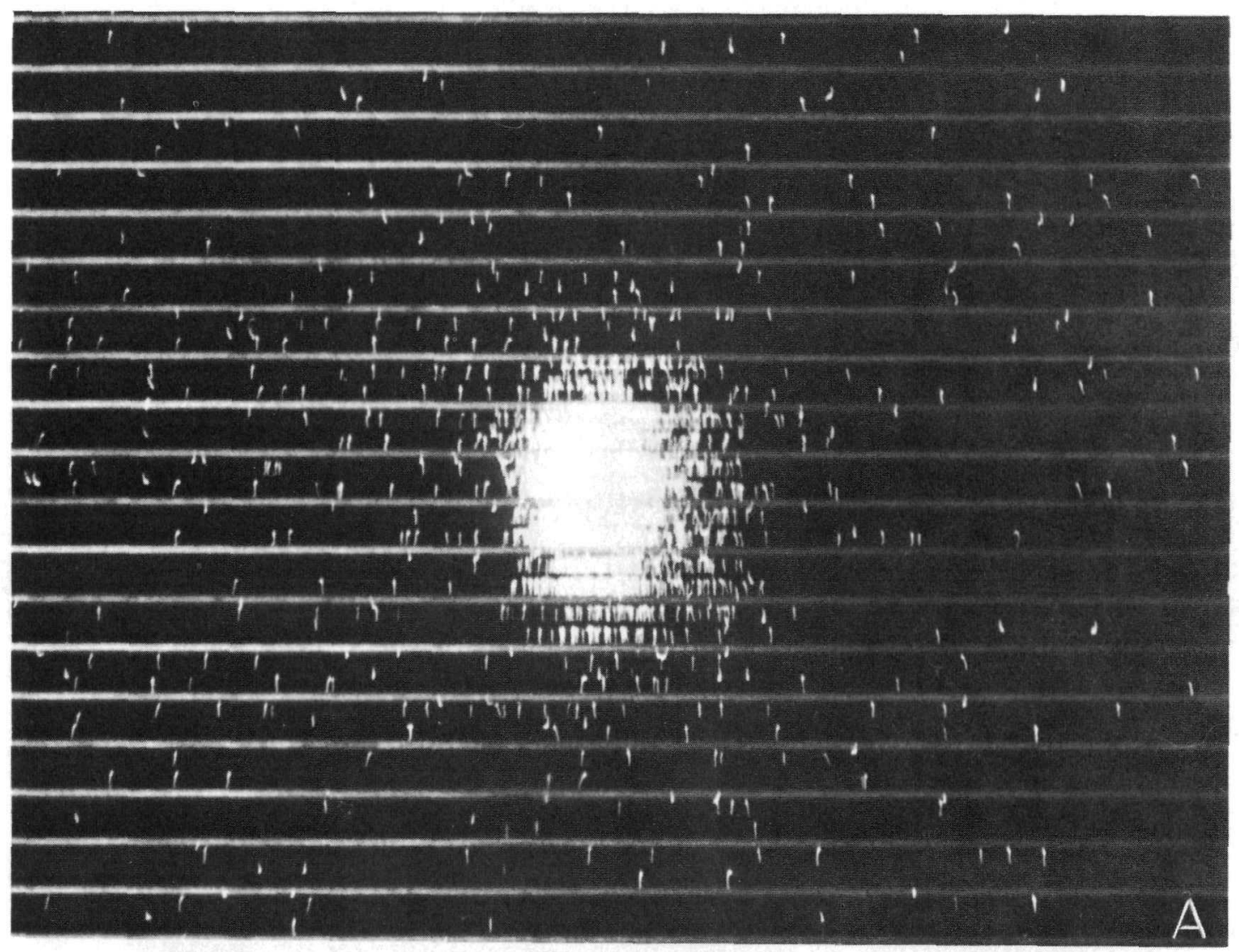

Fig. 5. Image Obtained with Multiwire Spark Counter for Circular Neutron Beam $6 \mathrm{~mm}$ in Diameter.

It is posible, and frequently desirable, to shift the spark counter systematically relative to a stationary radiation pattern during the image recording. By moving the spark counter, the effective resolution can be increased, since it is not so directly determined by the electrode spacing. 
Thus the area of the cathode wires in Fig. 5, which would be blank lines in a normal spark pattern due to the obscuration of the image by the relatively thick cathode wires, can be filled in by oscillating the counter during the exposure perpendicular to the wire axis over a distance equal to one-half the spacing of the cathode wires.

The resolution of the counter described above is about $1 \mathrm{~mm}$, as fixed by the electrode configuration. Investigations of the practical lower limits to electrode diameters and spacing for an attempt to increase the spatial resolution are being considered. In particular, operation under pressurized conditions is being considered with smaller electrodes. These devices can be made sensitive to protons and thus could be useable as fast neutron detectors with a proton recoil converter, such as a sheet of plastic.

\section{Semiconductor Particle Detectors}

Various semiconductors have been shown to detect charged particles. These semiconductor types include germanium- and silicondiffused pn junctions, gold-silicon surface junctions, and solid conduction detectors, such as sulfur crystals. Recently, the silicon detectors, both the phosphorous-diffused pn-junction type and the gold-silicon surface-barrier type, have been the subject of much research and development and practical use.(16) In general, these detectors are formed from silicon wafers about $1 \mathrm{~mm}$ thick. The sensitive volume is the depletion or space-charge region formed about the pn junction or barrier by the application of a reverse voltage bias to the detector. A charged particle creates hole-electron pairs in the depletion region, and a current pulse is formed across the junction by the collection of this charge. This current pulse produces a measurable voltage pulse, the height of which is proportional to the particle energy if the charged particle is completely stopped within the depletion region.

The more common silicon detectors available today are operable with depletion regions or sensitive regions that are several hundred microns in thickness. These devices are primarily used for the detection of the low-range charged particles, such as alphas and protons. For neutron detection, therefore, a $(n, \alpha)$ converter, boron-10 or lithium- 6 , is useable, and today one may purchase from various commercial sources semiconductor detectors prepared with neutron-conversion layers. Efficiencies for thermal neutron detection of about four percent are quoted by the suppliers. This is the detection efficiency expected from a $(n, \alpha)$-layer converter.

In general, these detectors are insensitive to gamma rays, but they will suffer an increased noise level in the presence of large $\left(>10^{3} \mathrm{r} / \mathrm{hr}\right)$ gamma-ray fluxes.(17) Recently, however, silicon-junction detectors are being made to function with increased depletion-layer thicknesses so that the devices may be used as beta-particle detectors. Mayer et al.,(18) have

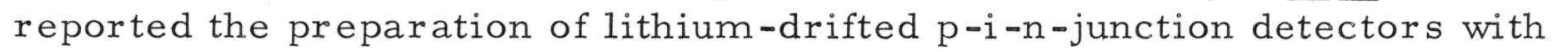


active volumes between $\mathrm{l}$ and $4 \mathrm{~mm}$ thick. The thicker detectors would, of course, have an increased gamma response, but these detectors could be used with the beta-emitting activation material converters which would give a better detection efficiency.

Dr. S. Friedland(19) has commented on the current state of development of diffused-junction semiconductor detectors for potential use in neutron imaging. He stated that there is currently no known way to obtain storage of the ionization in the detectors to allow an iconoscope type readout. Thus it would be necessary to arrange an array of these detectors with individual connections and amplification for each detector. Therefore, a unit $10 \mathrm{~cm} \times 10 \mathrm{~cm}$ of $1-\mathrm{mm}$ detectors with a $1-\mathrm{mm}$ spacing, i.e., l-mm resolution, would require 2500 individual detectors and amplifiers.

\section{Luminescent Image Detectors}

In detection techniques using film, neutron scintillators have been used for image detection. The scintillator which Berger(11) reported on is a mixture of boron-10 and $\mathrm{Zn} \mathrm{S}(\mathrm{Ag})$ in a plastic binder as developed by Sun et al.(20) More recently, Stedman(21) has reported on a thermal neutron scintillator made up of a mixture of lithium fluoride (96\% enriched lithium-6), Lucite, and $\mathrm{Zn} \mathrm{S}(\mathrm{Ag})$. Plates, $0.8 \mathrm{~mm}$ thick, of this mixture are reported to have an efficiency for neutron conversion of about 60 percent at thermal neutron energies. Slabs of these or other luminescent materials are applicable to both film-recording methods or could provide the input to any of the many electronic image-intensifier devices now becoming available. However, as these luminescent slabs become thicker for increased detection efficiency, the image resolution decreases.

The image resolution of luminescent slabs is generally considered to be about the thickness of the slab. This is illustrated in Fig. 6. In this figure we show the light distribution from an infinitely thin pencil of radiation impinging normally upon an optically homogeneous slab of thickness t. If there is no change in refractive index between the slab and recording device, so that no total internal reflection occurs at the exit face, then it is apparent that a significant fraction of the emergent light leaves the slab at very large distances from the center of the ideal image. If one includes the effect of total internal reflection caused by a refractive index step at the exit face of the slab, the intensity distribution is significantly narrowed, but unfortunately a large fraction of the otherwise useful light is lost. Ignoring Fresnel losses, it is easily shown that the fraction of potentially useful light actually escaping is given by $F=1-\left[\left(N^{2}-1\right)^{1 / 2} / N\right]$, where $\mathrm{N}$ is the relative refractive index of the slab. The dotted curve of Fig. 6 shows the relatively small change in the distribution if Fresnel losses at the exit face of the slab are also considered. 


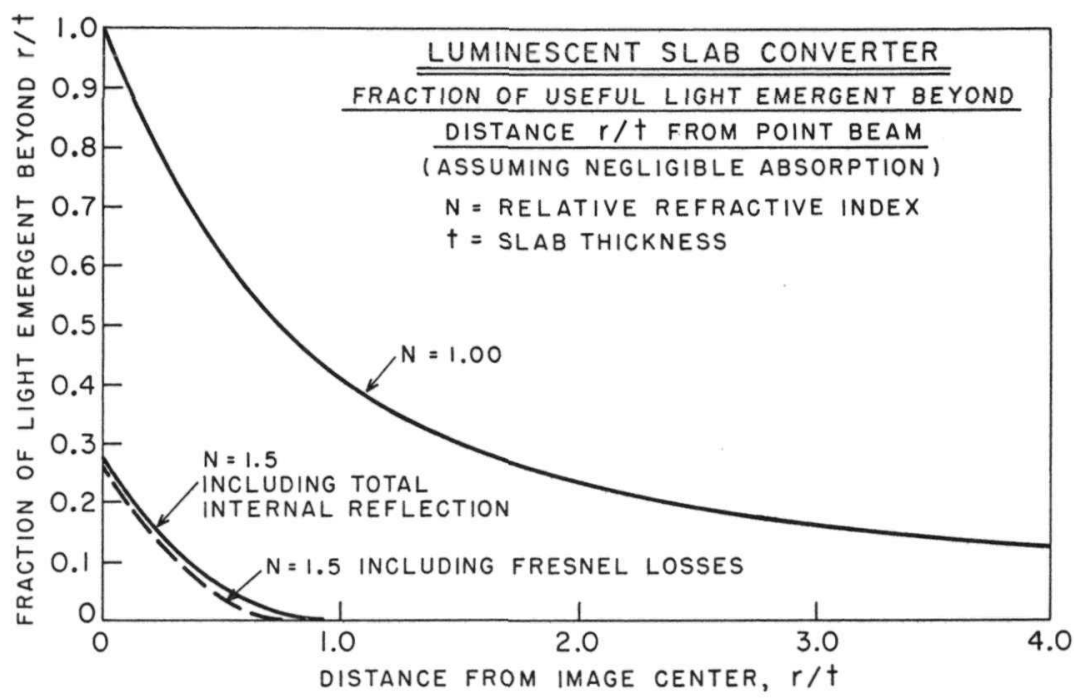

Fig. 6. Light Emergent from a Luminescent Slab Converter of Relative Refractive Index $\mathrm{N}$

The light-piping techniques developed in "fiber optics" and now applied to luminescent chamber development are obviously applicable to imaging. One can assemble a converter screen comprised of a closepacked array of optically insulated luminescent fibers of length sufficient to stop most of the incident neutrons. The resolution of the resulting optical image would be determined only by the diameter of the fibers employed and by the neutron scattering, both in the object and in the detector. Reiffel and Kapany (22) have presented graphs from which the performance of scintillating fibers can be estimated. These indicate that about 10 percent of the visible or ultraviolet photons produced in the luminescent fiber are trans mitted to the end of the fiber. An alternative to the solid rod or fiber is a stack of thin-walled glass tubes filled with a liquid neutron scintillator.

Another principle which would permit the use of very thick converter screens without attendant loss of resolution due to optical effects is shown in Fig. 7. This technique might be useful with detector materials which cannot be fabricated in the form of fibers of optical quality or for situations where only the converter screen and not the final imagepresentation device may be in place during the neutron exposure. The detector slab in Fig. 7 is a material which is either radio-photoluminescent or exhibits stimulated phosphorescence or fluorescence after being subjected to neutron radiation. An example of the latter type of material which is known to store a substantial fraction of the incident X-ray energy in sites which can be stimulated by optical irradiation is silver-activated sodium chloride.(23) An example of the former type of material is the silver phosphate glass developed by the U. S. Naval Research Laboratory(24) for purposes of radiation dosimetry. With either class of material a stimulating optical beam is used to read the information stored in the slab by previous 
high-energy irradiation. The resolution of the system is determined by the properties of the high-energy radiation per se and by the diameter of the scanning beam. All of the light emergent from the slab for a given position of the scanning beam contains useful information; hence, reflection or lens losses need not be sustained and read-out can be accomplished with available large-area photomultipliers rather than requiring large-area image-intensifier tubes. The read-out method can automatically compensate for distortions produced by a finite radiation source-to-object distance by arranging for the same divergence in the optical beam as was present in the exposure beam. Fiber arrays of thermoluminescent or photostimulated materials would also provide a good resolution in thick converters.
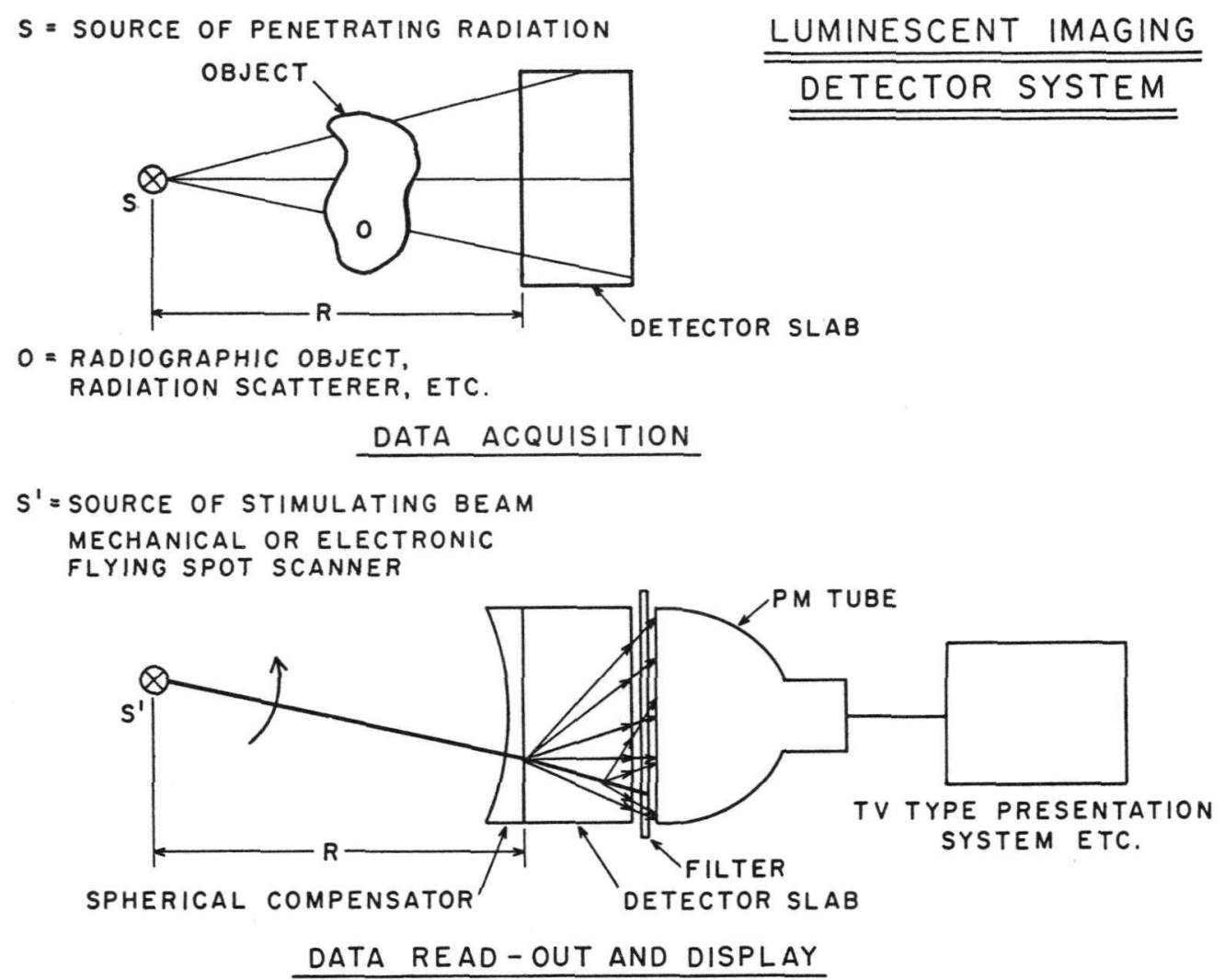

Fig. 7. Imaging Detector System Using Scanning Beam for Readout of Thick Detector Slab.

V. Neutron-Scattering Effects on Image Quality

To assess the effect of neutron scattering in a radiographic object on the image quality, neutron radiographs were obtained for an idealized object having known components of neutron absorption and neutron scattering. The neutron-absorption part of the object was a $\frac{1}{4}$-in.-wide $\operatorname{strip}$ 
consisting of 20-mil-thick cadmium and 25-mil-thick indium. Reactorgrade graphite (essentially a pure neutron scatterer with negligible absorption) was used as the scatterer portion of the object. The scattering mean free path of thermal neutrons in graphite is $2.6 \mathrm{~cm}$ (about one inch). Graphite slabs with dimensions of $16 \times 16$ in. and thicknesses varying from one inch to 6 in. were used.

The experimental geometry is shown in Fig. 8. A collimated neutron beam of $1-\frac{1}{4}$-in. diameter from an experimental port of the ARF Research Reactor was used as the neutron source. Four-mil-thick indium foils were used as neutron converters with an activation-transfer to type $\mathrm{KK} X-r a y$ film to eliminate the effects of gamma rays from the reactor.

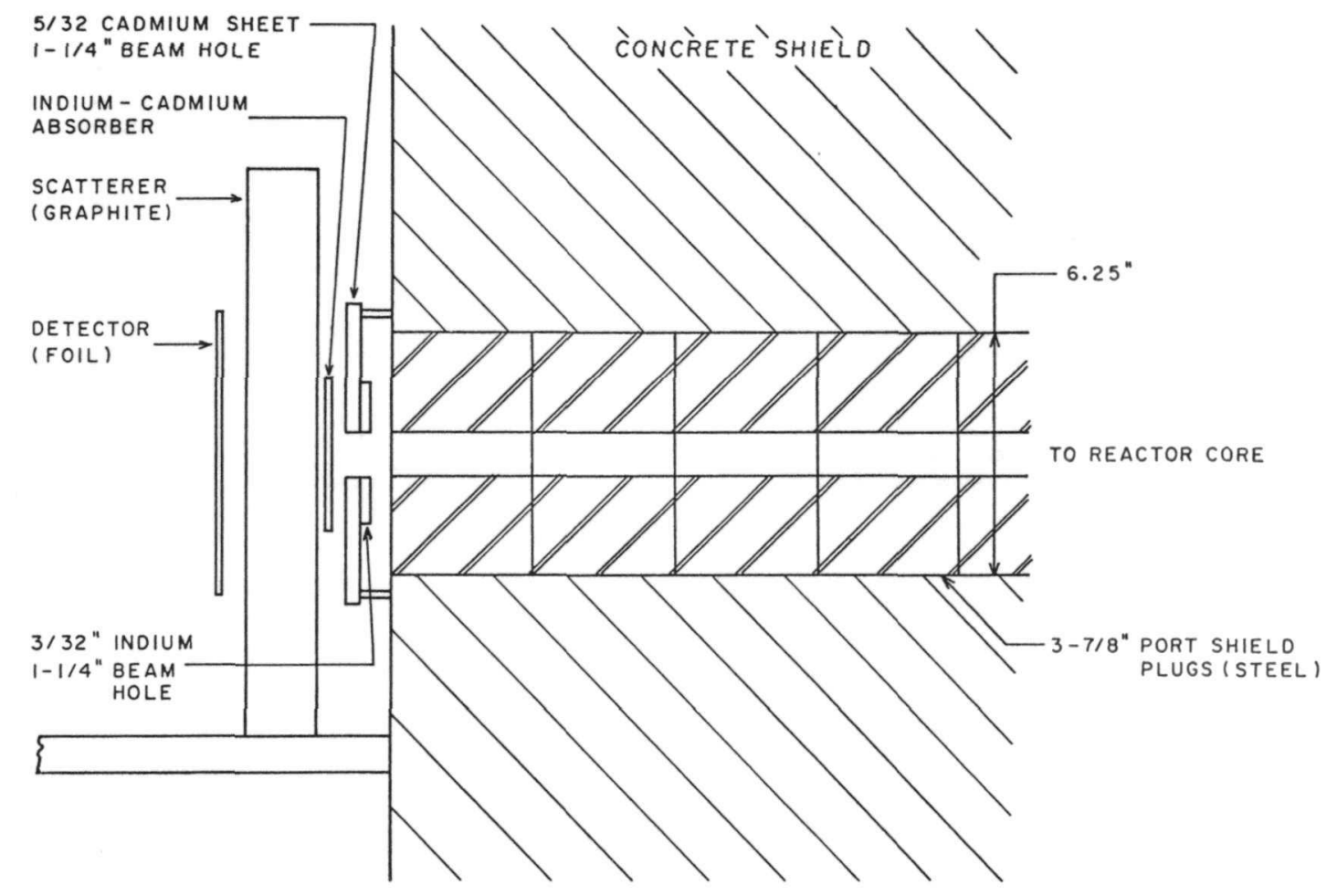

Fig. 8. Experimental Set-up for Neutron Exposures from Armour Research Reactor.

In Fig. 9 we show four different neutron radiographs of the absorption strip. The first radiograph is of the absorption strip with no neutron scatterer. The following radiographs have 1 in., 3 in., and 6 in., respectively, of graphite scatterer behind the absorption strip. In all cases the detector foil is placed $\frac{1}{4}$ in. behind the object. One can qualitatively observe the degradation in contrast and sharpness of the absorption strip image as the scatterer thickness is increased. 


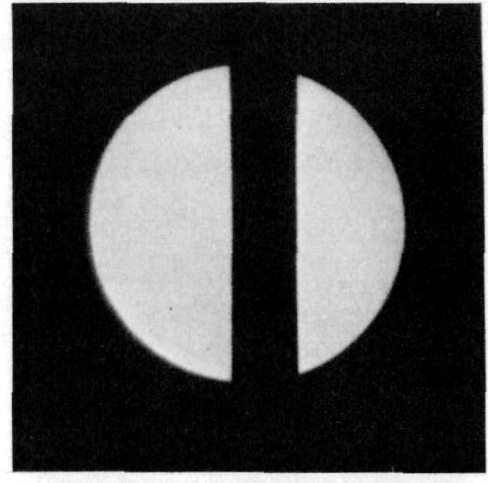

0 in. Graphite

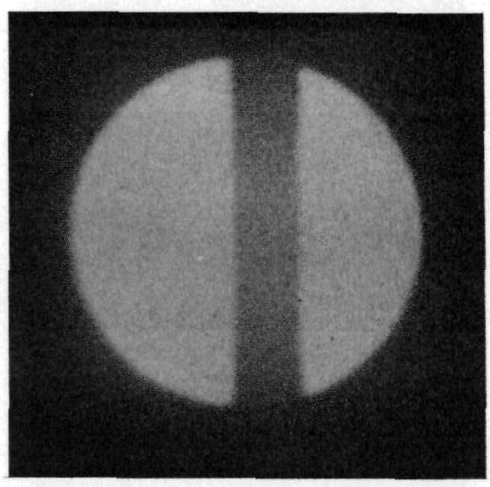

3 in. Graphite

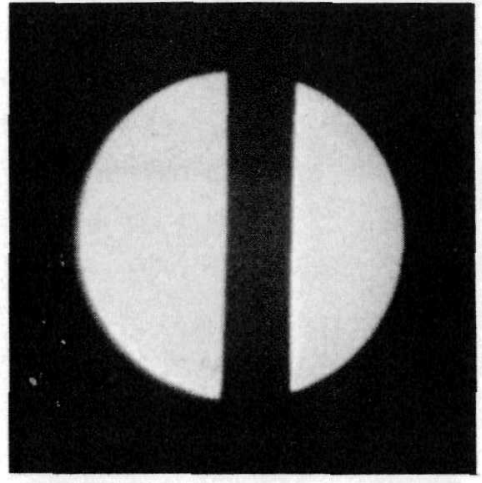

1 in. Graphite

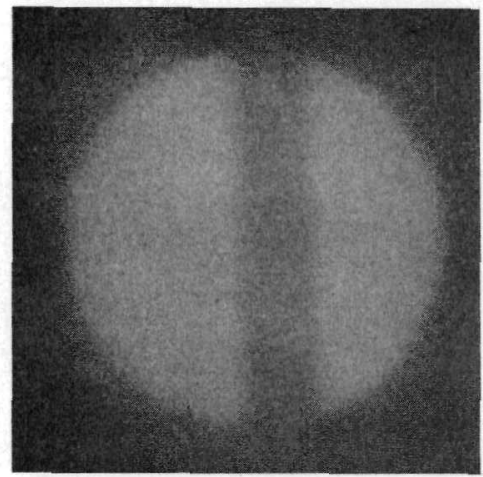

6 in. Graphite

Fig. 9. Neutron Radiographs of Cd-In Absorption Strip Followed by Various Thicknesses of Graphite Scatterer

The effect of the scatterer is shown more quantitatively in Fig. 10, in which we show the microdensitometer traces of the absorption strip edge. These curves were obtained with a Leeds and Northrup recording microdensitometer with a slit width of $0.05 \mathrm{~mm}$ and a slit height of $0.5 \mathrm{~mm}$. In all cases the 2 edges of the strip gave identical traces. The decrease in slope of the curves is an indication of the loss in sharpness, and the decrease in the difference of optical density is an indication of the los $\mathrm{s}$ in contrast as the scatterer thickness is increased.

As mentioned previously, we have used an optical system to simulate the effects of neutron scattering on the neutron-intensity pattern at the image plane. The following paragraphs describe the basis of this optical "image synthesis" technique and demonstrate the way in which it is used to study imaging system characteristics. 


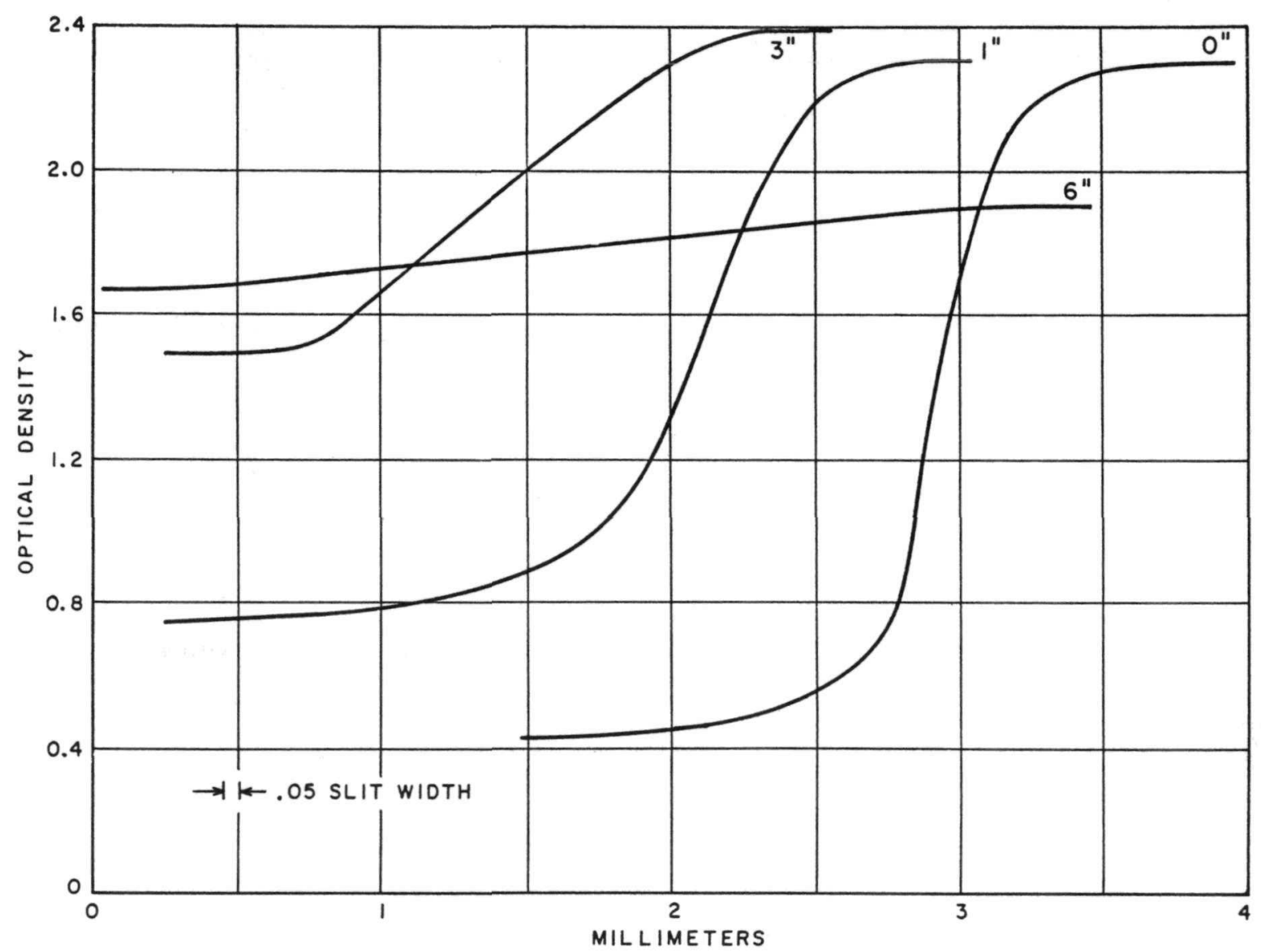

Fig. 10. Microdensitometer Traces of Cd-In Absorption Strip Edge

Image Synthesis by Optical Techniques

In the design of optical lenses, the so-called "spot diagram" is used to describe the imaging quality of the lens. This spot diagram is the locus of ray intersections at the image plane for rays from a single object point. It is evident that the spot diagram may include image-degrading effects other than lens aberrations, such as light scattering and diffusion.(25) The intensity function at an image plane for an extended object may be computed from this spot diagram or the image may be synthesized. A typical experimental arrangement of the image synthesizer consists of a diffusely illuminated plate having transparent spots in an opaque field representing the spot diagram. The light transmitted through the spots is then incident upon a test object, and a shadow of this object is cast at an image plane. It has been shown in the optical case that the image produced by this sys tem is almost indistinguishable from an actual image formed by a lens system with aberration characteristics equivalent to the spot diagram.(26)

The mathematical basis of the method of image synthesis is a convolution integral of an object function and transfer function: 


$$
\mathrm{I}(\mathrm{x}, \mathrm{y})=\iint \phi(\xi, \eta) \mathrm{T}(\xi, \eta ; \mathrm{x}, \mathrm{y}) \mathrm{d} \xi \mathrm{d} \eta \quad
$$

where $\mathrm{I}(\mathrm{x}, \mathrm{y})$ is the image intensity function at detector plane $(\mathrm{x}, \mathrm{y}) ; \phi(\xi, \eta)$ the object function at object plane $(\xi, \eta)$; and $\mathrm{T}(\xi, \eta ; \mathrm{x}, \mathrm{y})$ the transfer function from object to image plane.

In neutron imaging we have the following analogs for Eq. (1). The image function is the number of neutrons per $\mathrm{cm}^{2}$ per second impinging upon the detector plane at position $(x, y)$; the transfer function is the neutronscattering point spread function; and the object function is the true neutronabsorption component of the physical object. The scattering point spread function is derived from observing (analytically or experimentally) the image of a point beam of neutrons incident normally to a slab of scattering material. This scattering point spread function or transfer function is, of course, dependent on the thickness of the scattering material.

The synthesis scheme is illustrated in Fig. Il. Plates with patterns of the scattering spot diagrams are prepared for different scatterer conditions. The neutron-absorption pattern is prepared as a flat, optically absorbing object. The neutron image is then the shadow of this absorption object as diffuselyilluminated through the scattering plate. The physical sizes of the optical spot diagram, the optical absorber, and the distances $A$ and B of Fig. 11 are determined such that the magnified shadow size of the absorber, $d^{\prime}$, and the projection of the spot diagram size, $D^{\prime}$, are in the same ratio as their counterparts in the neutron geometry, i.e., $D^{\prime} / d^{\prime}=D / d$.

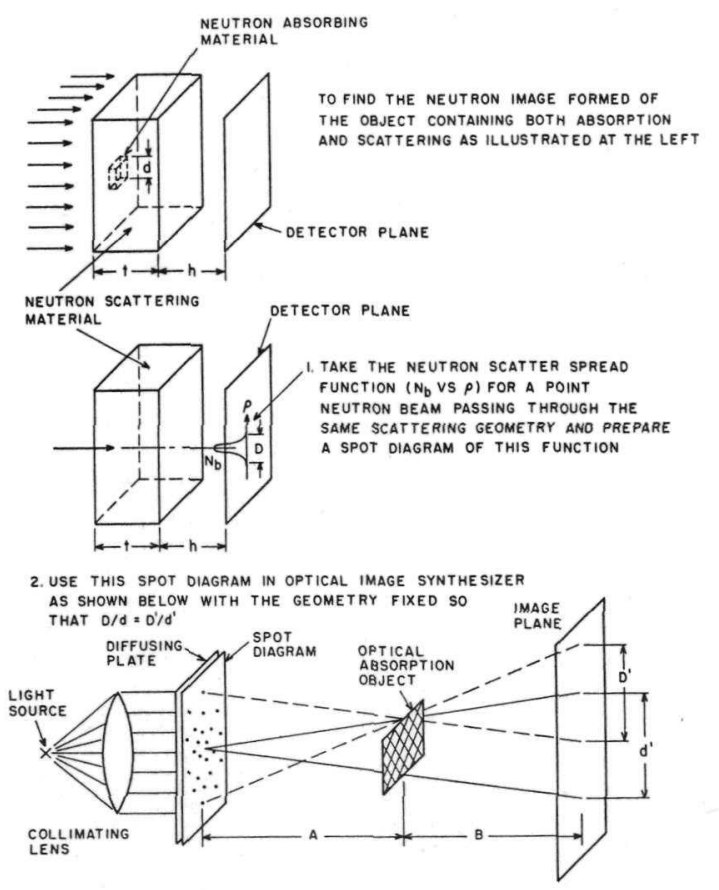

Fig. 11

Image Synthesis 
Both analytic and experimental methods for obtaining the neutronscattering point spread function have been used. Neutron-diffusion theory was used to derive analytically the number of scattered neutrons per $\mathrm{cm}^{2}$ per second emerging at a radial distance $\rho$ from the center of the neutron beam for an incident point thermal neutron beam on a semi-infinite scatterer slab of 6 scatter mean free path thicknesses. The distribution of the scattered neutron intensity emerging from a 6-in.-thick slab of graphite was experimentally measured for an incident collimated neutron beam of $\frac{1}{8}$-in. diameter. This beam size is small enough (about one-tenth of a scatter mean free path in graphite) that the beam size should not appreciably perturb the curve of scatter intensity versus position. A silicon-junction particle detector with a boron-10 neutron-converter layer and indium metal disks were used as neutron detectors in the experiments. Each detector had a sensitive area of 0.1 -in. diameter. The calculated curve and the experimental data were in reasonable agreement.

A graphical integration of the scattering curve is performed to determine the spot-diagram patterns. The neutron-scattering spread function is symmetrical about the beam axis; thus, one integrates this function over the area of revolution between two radii to obtain the correct weighting of the number of holes which are to be placed in the spot-diagram plate torepresent the corresponding area. The integration was divided into 6 intervals to include the scattering between $\rho=0$ to $\rho=6$ scatter mean free paths. On the spot-diagram plate, the holes are placed equidistant on circles with radii which are the centroids of the area of the annular rings. A hole is placed at the center of the diagram to represent the non-scattered neutron beam. To adjust the non-scattered beam intensity hole relative to the holes representing the scattering, we use an optical neutral density filter over the scattering holes.*

Spot-diagrams made with 10-mil-diameter holes in 10-mil-thick half-hardened brass plates were used in the optical bench set-up shown in Fig. 12. The light source and reflector is a standard dark-room, bullettype lamp with a diffusing screen of opal glass. The light intensity over the face of this screen is constant to within 3 percent. The spot-diagram plate and the neutral density filters are placed directly on the face of the diffusing screen. In the photograph, a photodetector on a scanning rig is set in the image plane; alternatively, a film pack may be placed at the image plane for image recording. The optical-absorption object is placed between the light source and image plane.

\footnotetext{
* A more rigorous discussion of this image synthesis is presented in ARF 1164-15, Research on Neutron Interactions in Matter as Related to Image Formation, Armour Research Foundation, Chicago 16, Illinois (October 1961).
} 


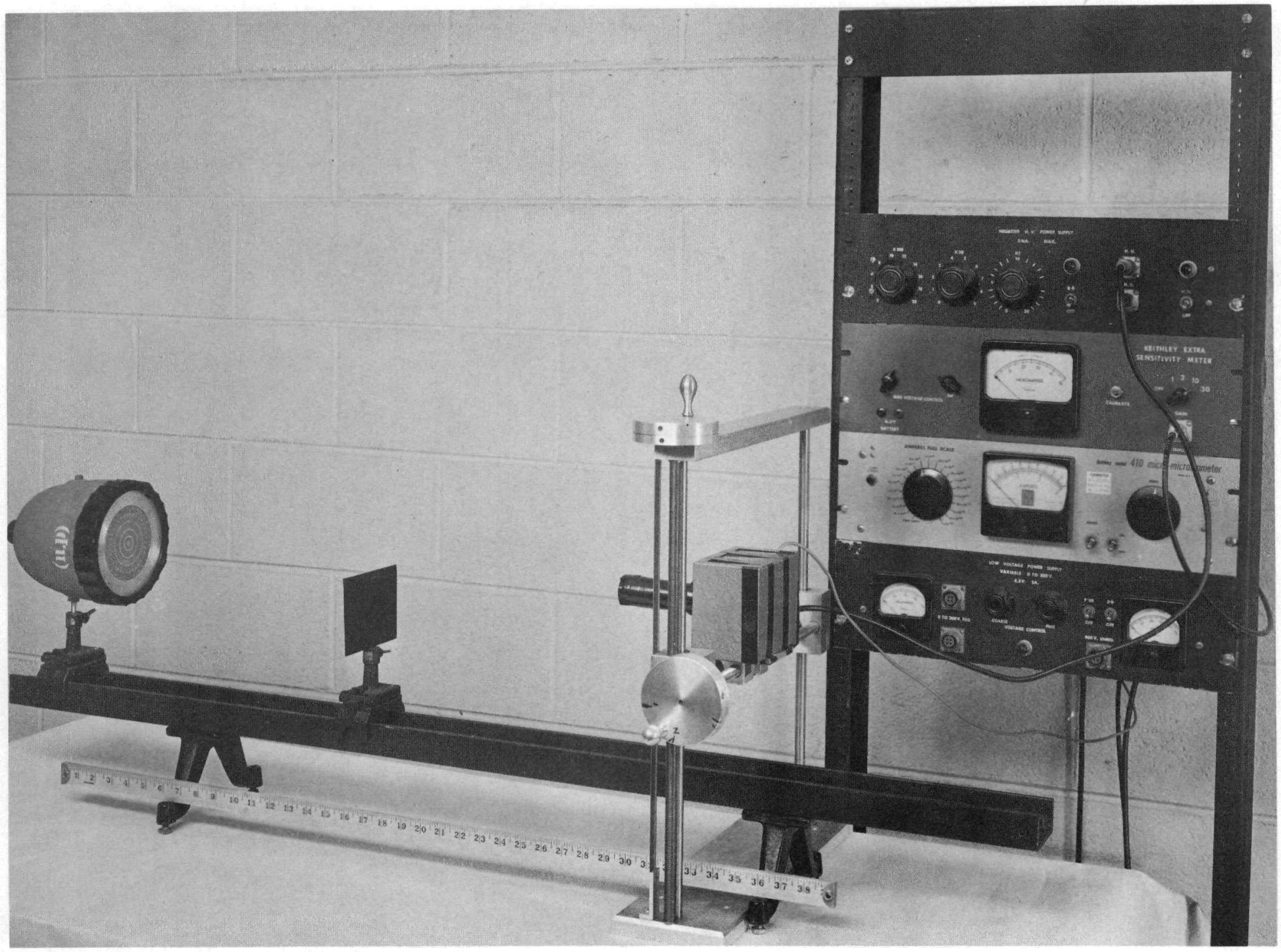

Fig. 12. Apparatus for Optical Image Synthesis 
Four synthesized images are reproduced in Fig. 13. The optical bench scaling was set to match the conditions of neutron scattering in graphite as shown in Fig. 9. The images were recorded on type KK X-ray film. The response of this film to the visible light intensities used in these experiments was found to be similar to the response curve for $\beta$ particles; thus the optical images can be compared with the neutron radiographs obtained on the same type film. Images are shown for an opaque $(0 \%$ transmission) filter over the scattering holes, and for filters of 2.25 percent, 4.75 percent, and 10 percent transmission over the scattering holes. The microdensitometer traces of the original negative of these films are shown in Fig. 14. As one would expect, the sharpness and contrast of the strip image decreases with an increase in scattering effect, i.e., an increased filter transmission.

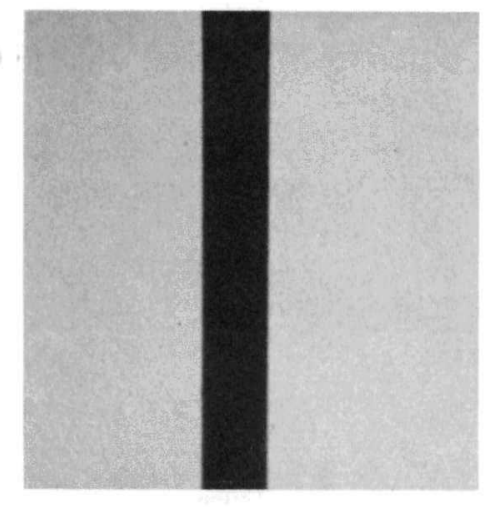

$0 \%$ Transmission Filter

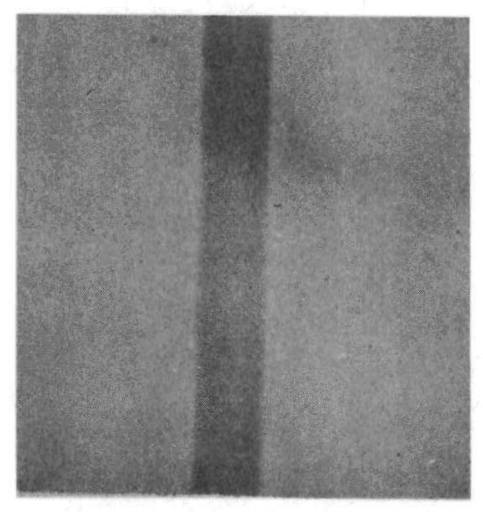

4. $75 \%$ Transmission Filter

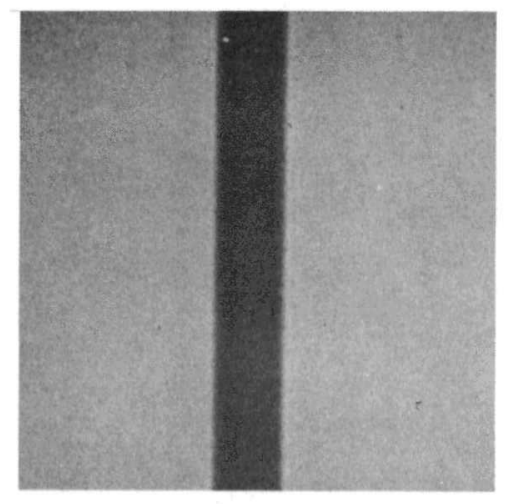

2. $25 \%$ Transmission Filter

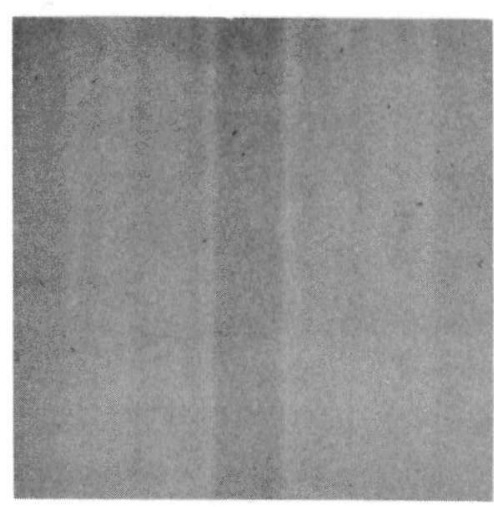

10\% Transmission Filter

Fig. 13. Optical Images with Various Filters Over Scattering Spot Diagram 


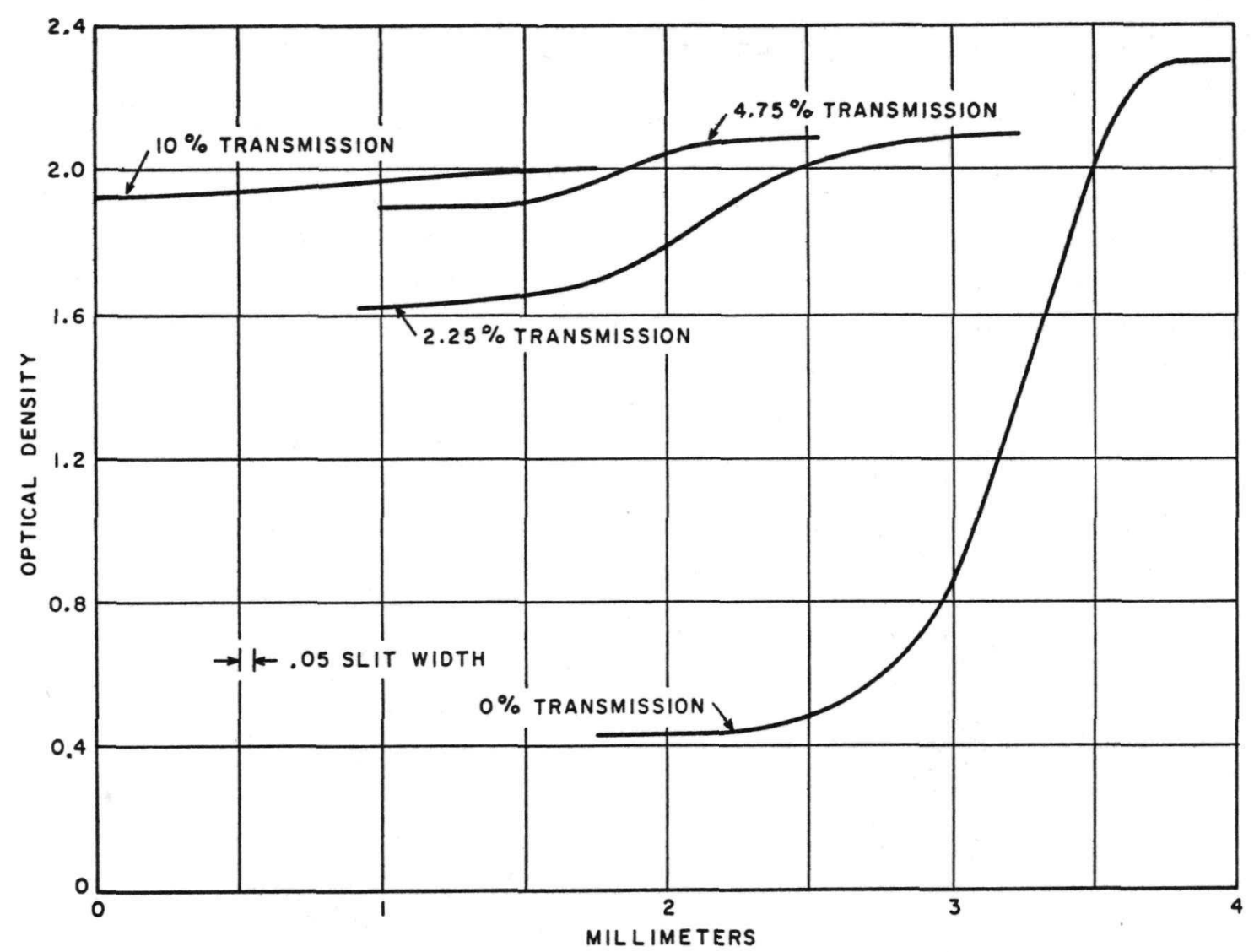

Fig. 14. Microdensitometer Traces of Optical Images

\section{Discussion of Experimental Results}

The characteristics of the microdensitometer traces chosen to describe the quality of the image are the slope of the curve at the midpoint and the total change in optical density. The slope is related to the sharpness, and the change of optical density is related to the contrast of the image. All exposures were made to give a nearly equal maximum optical density to all films used in our comparison, so that any nonlinear characteristics of the film would not alter the effects. The microdensitometer traces of the strip edge were taken at the central region of the neutron radiographs and on both edges of the strip in several cases. The curves obtained on the two opposite edges were symmetrical and reproducible.

The slopes and the optical density differences of the edges of the strip images from the neutron radiographs and some optically synthesized images are listed in Table I. The spot diagram used for the synthesized images corresponded to a thick scatterer and, indeed, when even a relatively small amount of "scattering" light was allowed ( $0.8 \%$ transmission filter), the slope decreased appreciably. It is seen that filter transmissions of about 2.0 to 10 percent give slopes which bracket those observed for the neutron radiographs with 3 and 6 in. of graphite scatterer. The opticaldensity differences across the edge are less for the synthesized images 
than for these particular neutron radiographs. This difference would, of course, be directly related to the amount of neutron absorption and in these radiographs a high-contrast neutron-absorption object $(20 \mathrm{mils}$ of cadmium plus 25 mils of indium) was used.

\section{TABLE I}

SLOPES AND OPTICAL DENSITY DIFFERENCES OF STRIP EDGE IMAGES FROM NEUTRON RADIOGRAPHS AND OPTICALLY SYNTHESIZED IMAGES

\begin{tabular}{|c|c|c|}
\hline \multicolumn{3}{|c|}{ Neutron Radiographs } \\
\hline $\begin{array}{c}\text { Thickness of } \\
\text { Graphite Scatterer } \\
\text { (in.) }\end{array}$ & $\begin{array}{c}\text { Slope at midpoint } \\
\text { of edge trace } \\
(\mathrm{OD} / \mathrm{mm})\end{array}$ & $\begin{array}{c}\text { Total change of } \\
\text { optical density } \\
\text { (across edge } \triangle O D \text { ) }\end{array}$ \\
\hline 0 & 3.74 & 1.88 \\
\hline 1 & 2.23 & 1.55 \\
\hline 3 & 0.66 & 0.90 \\
\hline 6 & 0.12 & 0.24 \\
\hline \multicolumn{3}{|c|}{ Optically Synthesized Images } \\
\hline \multicolumn{3}{|l|}{ Filter Transmission } \\
\hline 0 & 3.0 & 1.88 \\
\hline 0.8 & 0.8 & 0.57 \\
\hline 2.25 & 0.6 & 0.49 \\
\hline 4.75 & 0.3 & 0.18 \\
\hline 10 & 0.092 & 0.075 \\
\hline
\end{tabular}

The experimental data have shown that one can simulate the neutron intensity at the image plane for an object containing both neutron absorption and scattering by a relatively simple optical bench set-up. It is interesting to observe that one spot diagram is useable to produce a practical simulation for several scatter thicknesses by merely varying the relative amount of light transmitted through the "scattering" holes. We feel that a rigorous mathematical justification of these effects is not warranted at this time, and that it is sufficient now to use the simulation in an empirical and pragmatic fashion. It should be noted that such a technique should apply equally well to gamma-ray radiography studies in which gamma-ray scattering in the object is appreciable. 
The future use of the optical-image synthesis will be to study the effect of the detector spatial resolution on the image produced. This is performed by using a scanning optical detector in the image plane. An aperture equivalent to the proposed neutron detector spatial resultion is used with the photodetector.

\section{Conclusions}

The application of neutron imaging or radiography to complement $\mathrm{X}$-ray or gamma-ray radiography appears invaluable in many cases, especially when using thermal neutrons. Yet, at this time, we know of no routine use of neutron radiography. Today, there are small discharge-tube or accelerator-type sources of neutrons available; these produce neutron intensities sufficient to obtain neutron radiographs on X-ray film with activation foil converters within reasonable exposure times. The future applicability of isotopic neutron sources for neutron radiography appears promising, by virtue of improved neutron-imaging detection and/or isotopic neutron sources of higher yield.

An optical technique which simulates the neutron-intensity pattern at the image plane for a radiographic object containing both neutron absorption and neutron scattering has been developed. (Neutron scattering constitutes a prominent part of the neutron interactions in many materials.) On a simple optical bench set-up one can produce a simulated image which includes the effects of both the neutron scattering in the object and the resolution of the imaging detector.

One can consider the possible use of image detectors employing film techniques, arrays of individual detectors, thick luminescent materials, bundles of fibers or rods of luminescent materials, multiwire spark chambers, and optical image intensifiers or readout tubes in conjunction with the luminescent materials.

The future study of neutron imaging will be a logical continuation utilizing the now-proven optical-simulation technique to study the image quality or image information as the parameters of neutron scattering and image-detector resolution are changed. Data are being obtained at this time on the effect of detector resolutions of $0.25 \mathrm{~mm}, 0.50 \mathrm{~mm}, 1.0 \mathrm{~mm}$, and $2.5 \mathrm{~mm}$ on the edge image of an ideal absorption edge followed by a thick ( 3 to 6 scatter mean free paths) neutron scatterer.

\section{Acknowledgments}

The author wishes to acknowledge the assistance and cooperation of many Armour Research Foundation people in this investigation. In particular, the author would like to mention Dr. L. Reiffel and C. A. Stone for their 
encouragement and helpful discussions; Dr. T. G. Stinchcomb for his theoretical and analytical contributions during this study; and C. C. Preston and M. D. Oestreich for their assistance throughout this work. The cooperation of the Reactor Operations Section in providing the neutron facilities for this work is gratefully acknowledged.

\section{References}

1. Kallmann, H., Research 1, 254 (1947).

2. Peter, O., Naturforsch 1, 557 (1946).

3. Thewlis, J., Brit. J. App. Phys. 7, 345 (1956).

4. Berger, H., ANL-6346, 12 (1960).

5. Criscuolo, E. L., and Polansky, D., Missiles and Rockets Symposium, U. S. Naval Ammunition Depot, Concord, California (April 18, 1961).

6. Nucleonics 18 (12), 66 (1960).

7. Hennelly, E. J., Nucleonics 19 (3), 124 (1961).

8. Richmond, J. L., Group Leader, Mound Laboratory, Miamisburg, Ohio, private communication.

9. Nucleonics 14 (6), 105 (1956).

10. Price, W. J., Nuclear Radiation Detection, McGraw-Hill Book Co., New York (1958), p. 273.

11. Berger, H., "Neutron Radiography: A Second Progress Report," Symposium on Physics and Nondestructive Testing, Argonne National Laboratory (Oct 1961).

12. Hornyak, W. F., Rev. Sci. Instr. 23, 264 (1952).

13. Cohen, B. L., Nucleonics 8 (2), 29 (1951).

14. Cowan, F.P., and O'Brien, J. F., Proceedings of the International Conference on the Peaceful Uses of Atomic Energy, United Nations, New York (1956), 14, 213.

15. Reiffel, L., Rev. Sci. Instr.29, 1151 (1958).

16. Solid State Radiation Detection, IRE Trans. on Nuclear Science, Vol. NS-8, No. l (January, 1961).

17. Raymo, C. T., Mayer, J. W., Wiggins, J. S., and Friedland, S. S., Bull. Am. Phys. Soc., 5, No. 5, 354 (1960).

18. Mayer, J.W., Dunlap, H. L., and Wegner, H. E., Bull. Am. Phys. Soc. 6, No. 3, 313 (1961).

19. Friedland, S. S., President of Solid State Radiations, Inc., Los Angeles, California - private communication. 
20. Sun, K. H., Malmberg, P. R., and Pacjak, F. A., Nucleonics $14(7), 46$ (1956).

21. Stedman, R., Rev. Sci. Instr. 31, 1156 (1960).

22. Reiffe1, L., and Kapany, N. S., Rev. Sci. Instr. 31, 1136 (1960).

23. Furst, M., and Kallman, H., Phys. Rev.91, 1356 (1953).

24. Schulman, J. H., Shurcliff, W., Ginther, R. J., and Nucleonics, 11, (10), 52 (1953).

25. Kapany, N. S., Image Synthesis for Aerial Reconnaissance, ARF Project Suggestion No. 58-144AX (1958).

26 Keim, R. E., and Kapany, N. S., J. Opt. Soc. Amer., 48, 351 (1958).

\section{Discussion}

\section{INVESTIGATIONS IN NEUTRON IMAGING}

H. Berger, Argonne National Laboratory: Would you tell me a little more about the bor on spark-counter device? What was the thickness of the boron layer, and could you give any indication as to the speed of that device?

H. V. Watts: This was a layer of enriched boron-10, the thickness of which was $0.84 \mathrm{mg} / \mathrm{cm}^{2}$. You will recognize that number as being the range of the alpha particles produced from the boron reaction. This plate was prepared commercially. The spark counter has fairly uniform response and if we hit the plate with a uniform neutron intensity we get a fairly uniform spark pattern. It looks as if the plate coating is pretty good. Plate coating is a problem, especially in areas for which there are some $2,000 \mathrm{~cm}^{2}$ of boron coating. To answer the question of the speed - we used Royal-X-Pan and a camera setting at Fll. The spark counter operates at a voltage of 2100 volts in an argon-methane atmosphere; our neutron beam is of the order of $5 \times 10^{5}$ or $5 \times 10^{6} \mathrm{n} / \mathrm{cm}^{2} / \mathrm{sec}$. The image there was got in about $3 \mathrm{sec}$, and don't for get that this speed will be very dependent upon what you do with your optical camera. There is enough light intensity in each individual spark to really saturate an optical film.

G. M. Corney, Eastman Kodak Company: I think perhaps the agreement between the characteristic curve shapes for visible light of your X-ray film may have been fortuitous. If you were to change the intensity of your light by a factor of 2,3 , or 4 , you might find this curve shape no longer in agreement.

H. V. Watts: I purposely stayed away from saying too much about the film because I knew this audience contains many film experts. The agreement is probably fortuitous. We recognize the failure of the reciprocity. We have done some simple experiments of our own to try to see if we're way out of bounds on the se optical situations using that film. I know we're on tenuous ground here. 
THE LATENT IMAGE

by

\author{
Albert H. Allard \\ $\mathrm{X}$-Ray Technical Services \\ Eastman Kodak Company \\ Rochester, New York
}

\begin{abstract}
This lecture discusses the formation and subsequent development of the latent image. It traces the roll of gelatin in emulsion making, the structure of the silver bromide crystal, formation of sensitivity centers, and the electrical conductivity of the crystal as well as its role in the sequence of events producing the latent image. The effect of the latent image in relation to chemical processing is also discussed.
\end{abstract}

The subject of the latent image is a very complex one, and my intention is to avoid the many ramifications of the subject which would be of interest only to specialists in the field. Instead, a brief, accurate exposition of a currently accepted theory of latent image formation, surtable for background knowledge for the fields of photography and radiography, will be given. The formation of this image and its subsequent development are still the subject of intense research. This research is still attempting to demonstrate, extend, or disprove the currently accepted theories. And, as with many scientific theories, much of what is stated here may be obsolete tomorrow.

The latent image is important because it overcomes inefficiencies of the radiographic and photographic processes. This can be illustrated by considering what happens in X-ray production. When a beam of electrons is aimed at a focal spot, only one percent of the energy is utilized in the production of $X$ rays; the rest of the energy is converted to heat. Since these X rays are generated in all directions around the focal spot, only about one percent of the $\mathrm{X}$ rays actually go through the diaphragm towards the specimen. The other 99 percent is absorbed by the housing of the tube. Of the one percent of the one percent that finally gets to the specimen, only about one percent actually penetrates it; the other 99 percent is absorbed or scattered by the specimen. The one percent of the one percent of the one percent that finally gets through and hits the X-ray film is highly inefficient, and only about one percent of it is absorbed by the silver halide crystals and does useful work. The efficiency of this system is about $10^{-8}$. This is a very sad state of affairs. Some wit said that if you just squirted some X-ray energy on a silver bromide crystal to transform it to a visible particle of silver, the process of making a radiograph would take about 2,000 years. Production doesn't move fast at this rate. This is where the latent image becomes valuable. 
When radiant energy, whether $\mathrm{X}$ ray, light, or gamma radiation, strikes a silver halide crystal (silver bromide will generally be discussed, as it is the basic component of X-ray film), a change occurs which gives a latent or invisible or potential image. It is the fact that this latent image, in the presence of certain developing agents, can be converted to visible metallic silver particles in a relatively short time that makes the latent image and chemical processing important. With chemical development, this can actually be done in a few minutes, which gives us an amplification factor of $10^{9}$. It is this tremendous amplification that makes the concept of latent image and chemical processing so valuable by compensating for many of the inefficiencies of both the radiographic and photographic processes.

To understand the mechanism by which the latent image finally becomes possible, let us look at the cross section of an X-ray film. It is nothing more than a cellulose acetate base coated on both sides with an emulsion. It is double coated for two reasons: one is to decrease the exposure, and the other is to facilitate processing. Of course, the emulsions are coated with a protective coating to minimize abrasion. The cellulose acetate base is, primarily, a flexible support for the emulsion. It is the emulsion itself that is of the greatest interest. The basic components are gelatin and silver bromide crystals, but in any emulsion there are many other materials which are put in by the manufacturers to obtain different film characteristics.

The basic purpose of the silver bromide crystals is to supply particles of silver to make a visible image. The gelatin has a number of interesting characteristics, but there are two that are of concern. First, it is a vehicle in which silver bromide crystals are distributed. The trick, of course, is to distribute them equally and evenly throughout a film. The second property, and the one which has considerable value in latent image formation, is that the gelatin contains certain chemical substances which can sensitize silver bromide crystals, or any silver halide crystal, to radiant energy. In this case, it is the sulfur compounds in gelatin that make this possible. The X rays in some manner will then create a latent or invisible image which later we hope to make visible.

What do we mean by latent image? I think this is a very poor term in dealing with large groups of people with different backgrounds, because I find that many people seem to assume that this is some sort of complete image of an object which is just lying in wait for someone to make it visible. If one thinks of the total number of grains in a radiograph this is probably true, but I would like to limit this definition to one dealing with a single crystal. The latent image is defined as a speck of silver on a silver bromide crystal which is capable of initiating the development of this crystal. 
This is rather elementary, but as problems do seem to be encountered at this point, I shall quickly go through a very simplified concept that will lead to the formation of a silver bromide ion. The atom is a very complex thing, but basically, it is a nucleus containing protons or positive charges surrounded by shells containing electrons or negative charges. In the case of the silver atom there are 47 protons and 47 electrons. As it is only the outer ring that participates in chemical combination, we can simplify this structure by taking 46 protons and pairing them off with 46 electrons so that we have just the net effect of one proton in the center and one electron in the outer shell. The atom is still electrically neutral. This simplified concept when applied to bromine gives 7 electrons in the outer ring and 7 protons in the center for an electrically neutral atom. If an atom of bromine and silver come into close approximation to each other, the single electron of the silver atom can become a part of the electron shell of the bromine atom. When this happens they are held together by an attractive force, and now the silver atom is minus one electron and has a surplus positive charge in which form it is known as a silver ion, and the bromide having taken up an extra electron now has a surplus negative charge in which form it is known as a bromine ion. We have now a molecule of silver bromide. In a crystal of silver bromide there may be as many as one billion pairs of these ion structures in which the silver and bromide ions alternate.

In some of the interstitial spaces between the silver and bromide ions are discrete particles. These are the sulfide specks or sensitivity

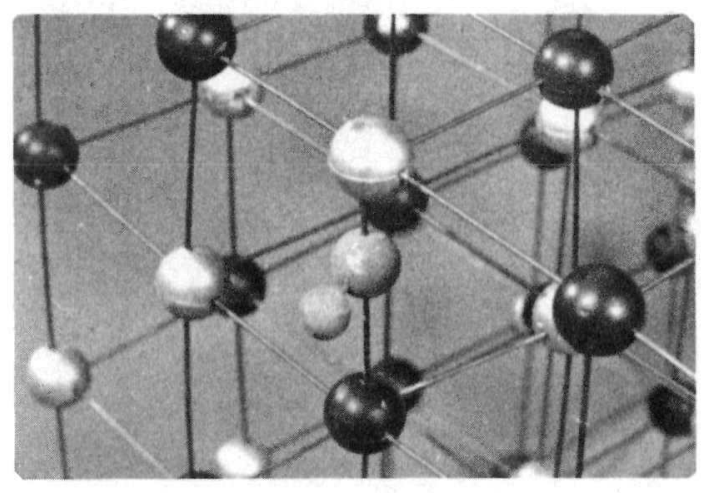

Fig. 1. Sensitivity Speck in Interstitial Spaces in $\mathrm{Ag} \mathrm{Br}$ Crystal specks which will help to sensitize this crystal to radiant energy. This is shown in Fig. 1. How they get there is a function of making emulsions.

In the preparation of an emulsion, the silver bromide is precipitated by reacting silver nitrate and potas sium bromide in dilute gelatin. The next three steps consist of ripening, gelation, and washing. Briefly this is what occurs. In the ripening, the size and structure of the crystals are controlled. The nature and concentration. of the gelatin affects the physical size and additional gelatin can be added to get the size desired. The emulsions are then washed to get rid of any undesirable products. It is the afterripening that's important in latent image formation, because it is at this point that sensitivity specks are produced. These specks are produced because the sulfur compounds which are a natural component of the gelatin 
can and do unite with silver to form silver sulfide specks or, as they are also known, concentration specks. It should be pointed out that this is not the only chemical sensitizer which is known, and that in addition to chemical sensitizers certain physical defects can also act as sensitivity points. The basic mechanism of latent image formation appears to be the same in all cases.

Now, one would suspect that with a crystal containing positive and negative charges that there would be some electrical conductivity, and it is true that in silver bromide crystals there is both ionic and electronic conductivity. The ionic conductivity occurs in the dark and is subject to temperature effects, whereas electronic conductivity is relatively independent of temperature. Ionic conductivity consists of the motion of interstitial silver ions. What are these interstitial silver ions, and how do they arise? During crystal formation certain effects may occur which lead to a silver ion being forced out of its normal position, and it then becomes free to migrate slowly within the crystal as an interstitial silver ion. This is

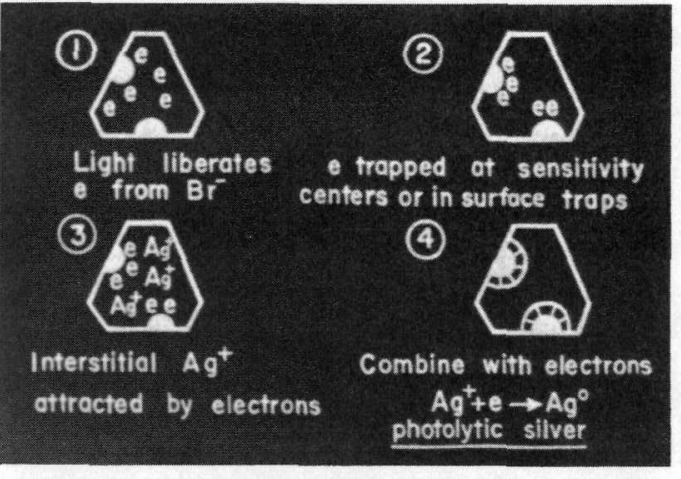

Fig. 2. Formation of Latent Image Nuclei ionic conduction and it is an important factor in the mechanism of the latent image. Electronic conductivity occurs as soon as a crystal is exposed to light or $\mathrm{X}$ rays or gamma rays. The radiant energy strikes the bromide ion and liberates an electron which then can move rapidly throughout the crystal.

The movement of ions and electrons through the crystal is the starting of the latent image mechanism. The electron which has been released can be attracted to the silver sulfide sensitivity specks, or to surface crystal defects, be trapped there, and give the trap a strong negative charge. This trap with a strong negative charge can attract the moving interstitial silver ions. If the silver ion comes into the trap it can then take up the electron in this trap to become neutral, forming an atom of silver. If this occurs, another electron may be trapped, another silver ion attracted to it, and neutralized to an atom. If this process is repeated a sufficient number of times, you will have latent images which will be stable and development can occur.

It should be pointed out that there can be more than one latent image because there are many sensitivity traps. Figure 3 is an electron micrograph in which development was started very minutely, and then stopped. At certain points, depositions of silver can be seen. These are points at which latent images existed. As this crystal had a low exposure to radiation there is only one or two latent images. However, at a higher intensity of exposure (see Fig. 4), the crystals have 4, 6, or 8 latent image centers which can initiate development. 


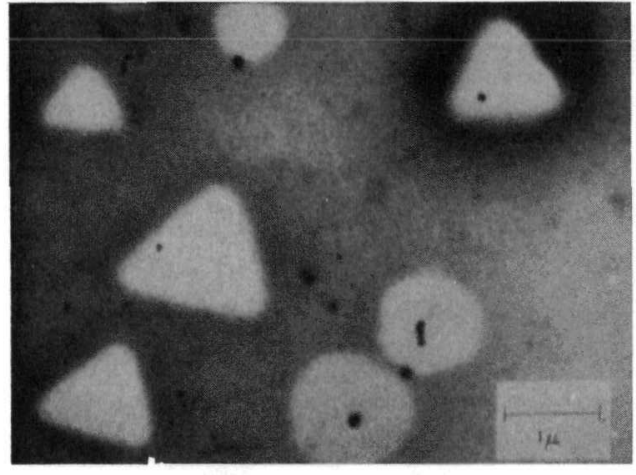

Fig. 3. Latent Image Centers: Low Exposure

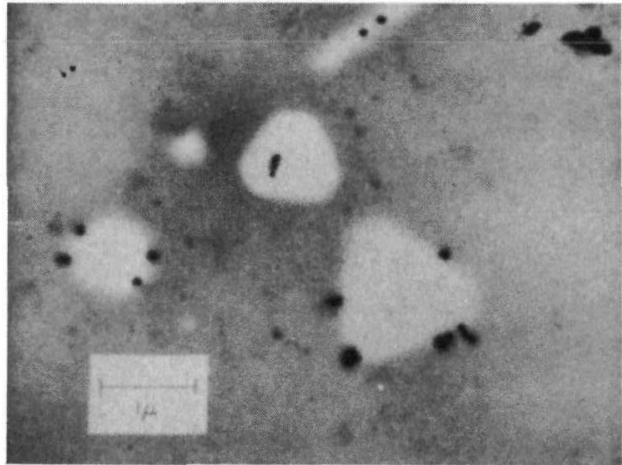

Fig. 4. Latent Image Centers: High Exposure

I shall just summarize this briefly. When light or radiant energy strikes the crystal of silver bromide, an electron is liberated from the bromide ion. This electron can be trapped at sensitivity centers which can be either physical or chemical, and the strong negative trap can then attract the interstitial silver ion; this ion combines with the electron and is deposited as an atom of silver. If we get several atoms of silver, we have a latent image.

Now, we defined a latent image as a speck of silver on a silver bromide crystal which was capable of initiating development of the crystal. This discussion, then, would not be complete, if it did not carry through to what happens in the development process and its relationship to the latent

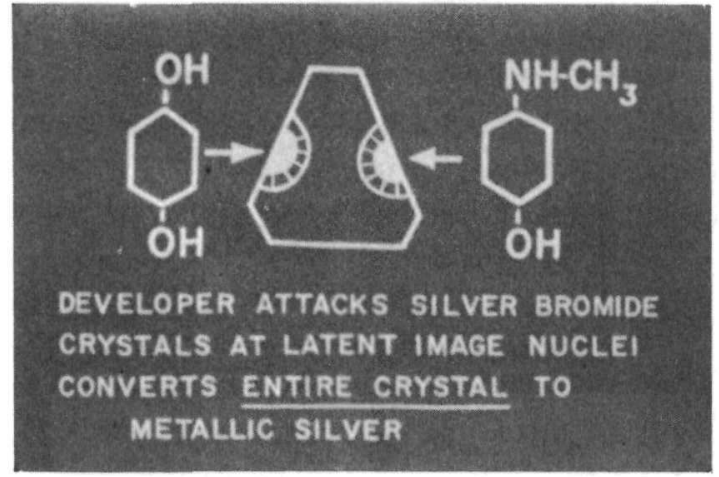

Fig. 5. Developer Attacks AgB.r Crystal at Latent Image Nuclei. Converts Entire Crystal to Metallic Silver. image. There is a lot we don't know yet, but, in general, we find that the developers will attack the silver bromide crystals at the latent image centers and convert the entire crystal to metallic silver. In short, the latent image becomes a sort of keyhole through which we can open the door to the center of the crystal. The thing that is important about the organic developing agents is that they are good electron donors. When they are put into solution, particularly an alkaline solution, they can give up electrons with considerable rapidity. These electrons are then free to migrate to the latent image centers, and reduce the interstitial ions, and the ions of the structure itself, to atoms of silver until the entire crystal has been broken down. The reduced crystals are held in place by gelatin, and at the end of the proper developing time, if all went well, you have a radiograph. 
Discussion

THE LATENT IMAGE

W. Z. Leavitt, Watertown Arsenal: What's the photo efficiency? What is the energy required?

G. M. Corney, Eastman Kodak Company: The invisible light will excite about one silver atom per photon absorbed in the individual crystal.

W. Z. Leavitt: What sort of cross sections do you have here?

G. M. Corney: I couldn't say.

L. A. Turner, Argonne National Laboratory: The latent image, the speck of silver, is the center that catalyzes the development. I can't help wondering why a developer which is so generous of electrons needs that speck of silver in order to give electrons up and do the developing.

A. H. Allard: It doesn't have to have the latent image in order to give up electrons. It would do this in solution anyway, but it is the electrons being catalyzed at the latent image centers which leads to the breakdown of the crystals. Apparently, the electrons without the latent image from the developer have no effect on the crystal structure.

C. A. Arenberg, Argonne National Laboratory: Are the first 3 atoms of silver considered the threshold value?

A. H. Allard: This seems to be true. Apparently, this tends to vary with different types of emulsions, and I'm sure we could get lots of arguments among the experts in the field of the latent image. There may be lower thresholds. At any rate, below 3 it does not stay stable and apparently has no value.

R. G. Peterson, Argonne National Laboratory: You mentioned that gelatin had an important role. Is the only function of the gelatin, besides the support of course, to supply these impurity sites, the sulfur compounds, or are there some other functions?

A. H. Allard: This is one of its major functions. However, gelatin has some very interesting characteristics; it is also a good bromine absorber. When we have an electron knocked off from the bromine, what happens is that the bromine then is in ionic form and is what is known as a hole. Now, another electron from an adjoining bromine can migrate to that hole, creating a hole behind it. Eventually, if everything goes right, these bromine atoms should end up at the gelatin where they will be absorbed. If this failed, of course, then you might have recombination of the electrons with this and no action. 
THE MEANING OF CHARACTERISTIC CURVES

by

\author{
E. T. Larson \\ and \\ H. F. Nitka \\ Ansco \\ A Division of General Aniline and Film Corporation \\ Binghamton, New York
}

\begin{abstract}
The characteristic curve of an X-ray film can be used directly to predict the rendition of large-scale features in the subject. For small-scale features it is found that the statistical fluctuations in the exposing radiation may have a material effect on the rendition. The effects of the statistical fluctuations are computed from the characteristic curve and are found to agree with experimental observations on actual radiographs.
\end{abstract}

\title{
I. Introduction
}

The characteristic curve of an X-ray film describes quantitatively the relationship between the $\mathrm{X}$-ray exposure and the optical density of the developed film for a specific set of exposing and processing conditions.

Figure 1 presents the characteristic curves for 4 typical industrial X-ray films. The films were exposed at $65 \mathrm{KVP}$ without screens and the exposure, measured in roentgens, was varied by changing the exposure time. The films were processed according to the manufacturer's recommendations, which is generally a good procedure. The density values of the processed films were measured with a densitometer calibrated in accord with the definition:

$$
\text { Density }=\log _{10} 1 / \mathrm{T},
$$

with $\mathrm{T}$ the optical transmittance.

The characteristic curve of an X-ray film is an extremely useful tool for the film manufacturer to use in measuring and controlling the properties of the film. Unfortunately, this particular method of quality control is not a nondestructive test. 


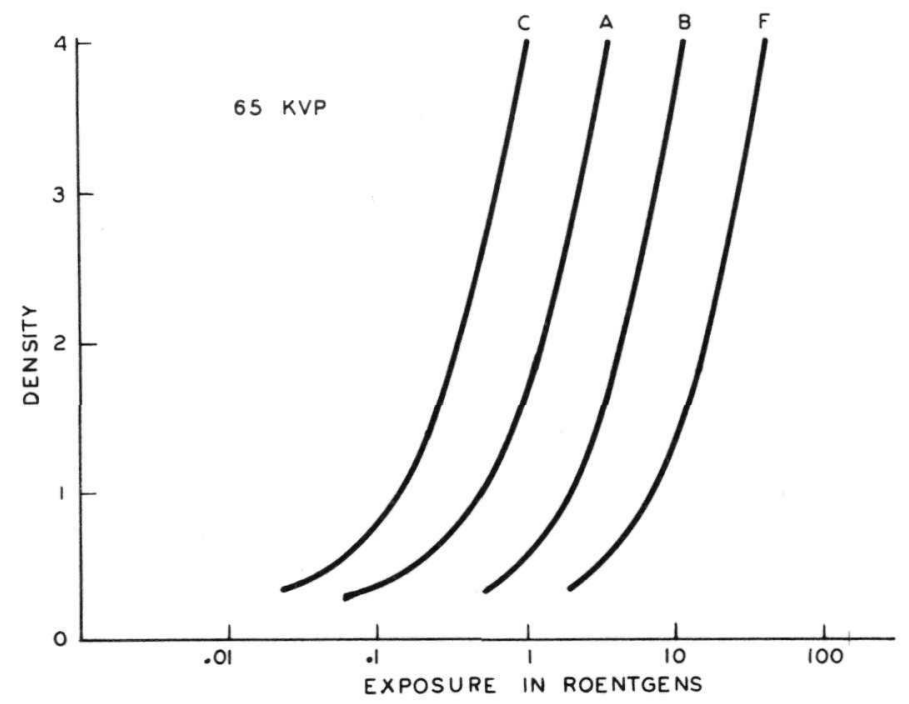

Fig. 1

Characteristic curves for four typical industrial X-ray films exposed to direct $X$ rays at $65 \mathrm{KVP}$. The principal differences among the curves are their positions along the horizontal axis.

In radiography, the significance of the characteristic curve has many ramifications, some of which are of greater practical value than others. In this discussion we shall devote out attention to the relationship of the characteristic curve to two aspects of radiography:

1. the depicting of large-scale or macro features of the subject being radiographed;

2. the depicting of small-scale or micro features.

\section{Rendition of Large-scale Features}

The characteristic curves in Fig. 1 were obtained by exposing separate areas of each film for various lengths of time to radiation of a fixed quality, namely 65-KVP, unfiltered $\mathrm{X}$ rays. The exposure was measured in roentgens with an $r$-meter placed in the plane of the film during exposure. The shapes of the curves are nearly identical for all of the films; the major differences among the curves are in their positions along the exposure axis.

If this experiment were repeated, using radiation of a different quality, such as $200-K V P X$ rays or the gamma rays from cobalt-60, the curves would have very nearly the same shape as in Fig. 1, but their positions along the exposure axis would change and the separations between the curves would change the intensity of the radiation, in roentgens per unit time, and the exposure time would have no effect on the shapes or positions of the curves.*

* The discussion here is limited to direct exposure by $\mathrm{X}$ rays, with or without lead screens. For exposures with luminescent screens, i.e., screens whichemit light, such as calcium tungstate screens, the reciprocity law does not hold, and film response then varies with the intensity of the radiation. 
It is also of value to present the data from Fig. 1 in another form, by plotting the exposure on a linear scale instead of a logarithmic scale, as is done in Fig. 2. The straight lines of Fig. 2 illustrate another wellknown property of $\mathrm{X}$-ray films exposed to radiation of a fixed quality, namely, that the density of the developed film is a linear function of the $\mathrm{X}$-ray exposure. This linear relationship is strictly true at low densities and is correct as a first approximation for densities up to about 3.0, except for films designed for use primarily with luminescent screens, for which the maximum density is usually less than 4.0. All of the films described here have a maximum density of 10.0 or higher.

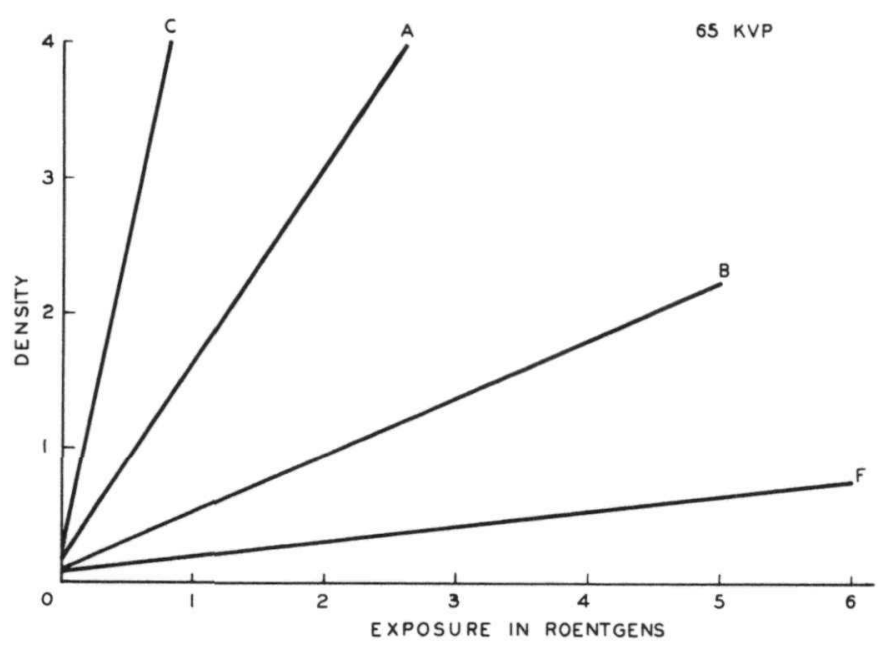

Fig. 2

Characteristic curves for the films of Fig. 1 but with the exposure plotted on a linear scale. For the practical range of densities, density is approximately linearly proportional to the exposure.

In the practice of radiography, the exposure and the quality of the radiationvary from area to area over the film, corresponding to variations in thickness, shape, and composition of the subject, and also to the amount of scattered radiation. Under these circumstances, it is convenient to represent the performance of the system by expressing the density of the developed film for various thicknesses of a particular material. Fig. 3 presents such a relationship for steel radiographed at $200 \mathrm{KVP}$ on film A.

Fig. 3

Relationship between density on film A and thickness of steel for an exposure of 33 milliampereminutes at $200 \mathrm{KVP}$ with lead screens. This has been called the characteristic curve of the radiographic system.

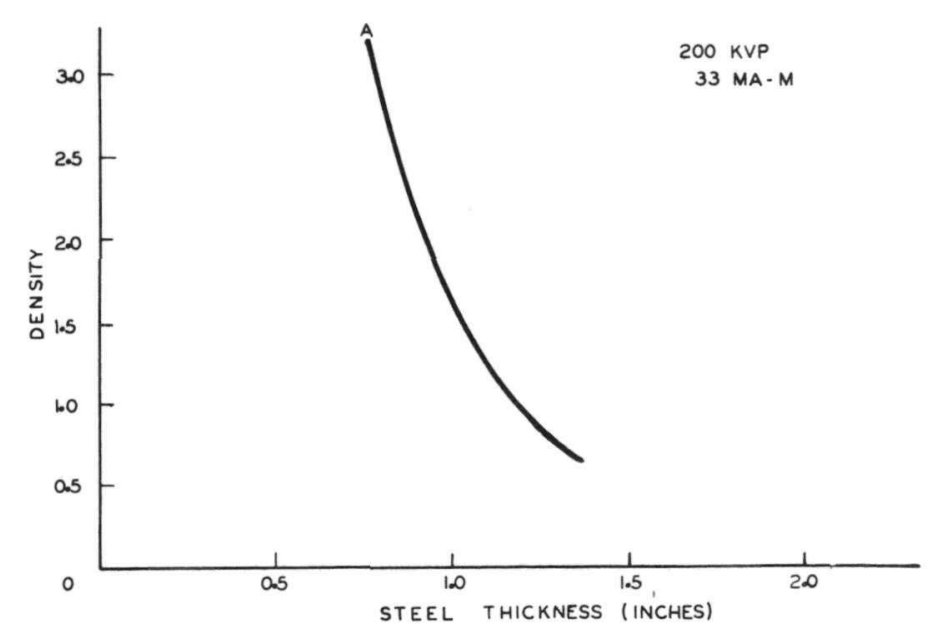


The form of this curve depends on the characteristics of the film. It also depends on other factors, such as the change in quality of the radiation in the direct beam with thickness and the amount of scattered radiation. This type of curve, therefore, may be termed the characteristic curve of the system or, more commonly, a technique curve.

A collection of curves such as those in Fig. 3 is usually used in one form or another in the practice of radiography. Figure 4 is an example of such a collection arranged as a slide rule for determining the density for various exposure conditions, types of material, thicknesses, and films.

It is important to note that the characteristic curves for films, characteristic curves for radiographic systems, and technique charts are customarily determined by measuring the densities of large, uniformly exposed areas of film. Therefore, the technique charts can be used to predict the rendition of only the large-scale or macro features of the subject. Through their use the radiographer can choose the film and technique which will produce the desired information about the macro nature of the subject.

In those instances where small-scale or micro details are of interest, it is necessary to give further consideration to the meaning of the characteristic curve of the film.

\section{Rendition of Small-scale Features}

The ability of a radiographic procedure to depict the features of a subject can be expressed in terms of the density difference $\Delta D$ produced by a thickness change $\Delta t$. It is a common practice in the literature to derive the value of $\Delta \mathrm{D}$ from a curve such as that in Fig. 3 by computing the corresponding value of $\Delta t$ at a particular value of $t$.

However, such a procedure is valid only for large areas in the image, for example, areas equal to or larger than the area of the light beam in the densitometer (usually about $3 \mathrm{~mm}$ in diameter) which was used to measure the density values represented by the characteristic curves or the technique curves.

Because of the quantum nature of $X$ rays, the density of a small area is not a unique or single-valued function of the exposure. Therefore, Fig. 3 cannot be used directly to predict the density in a small area of the image. Instead, it gives only the most probable value. For a given exposure and thickness, the density of a small area in the image will have a range of possible values, and this range will increase as the size of the area considered decreases. 


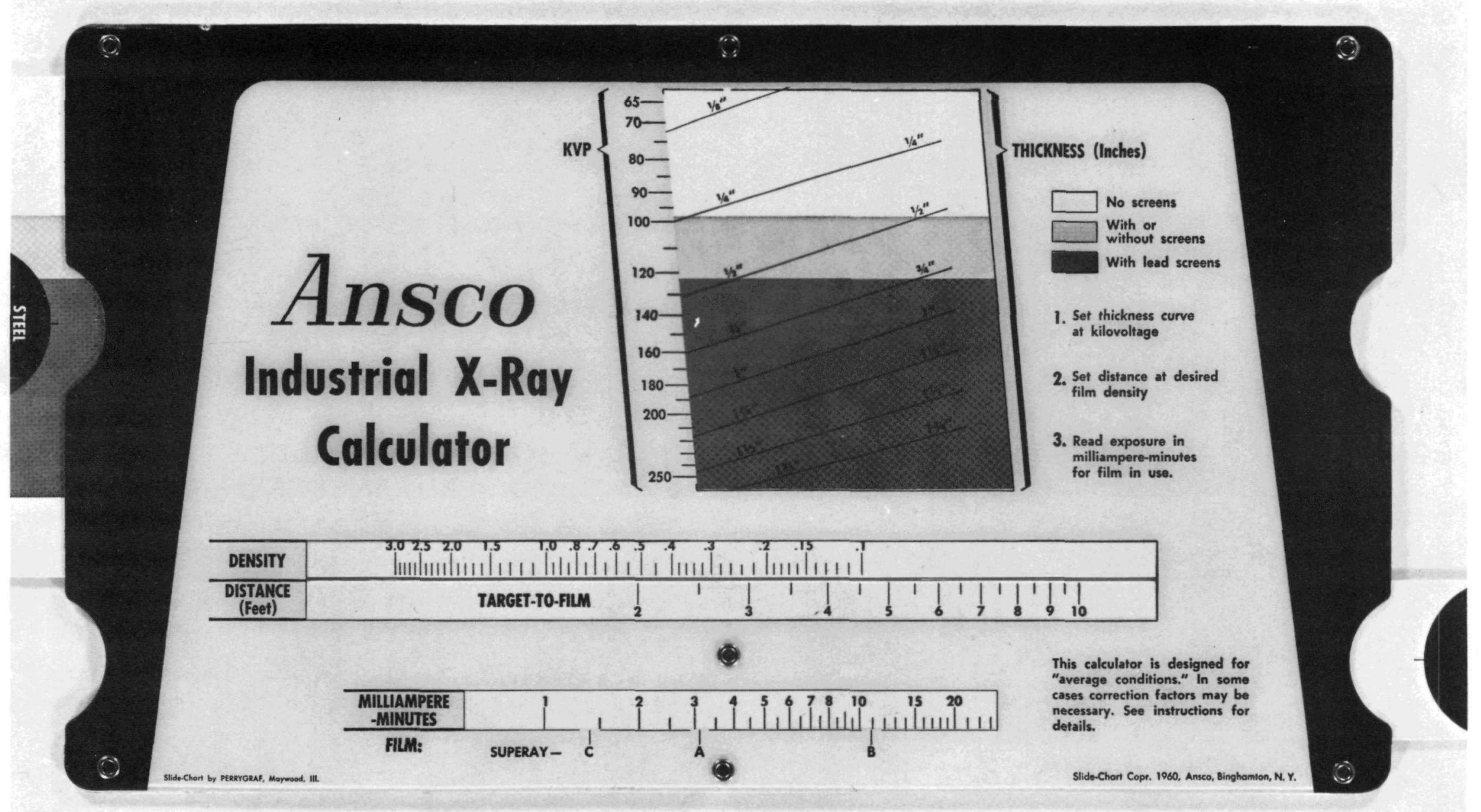

Fig. 4. Slide Rule for Industrial Radiography. 
The fact that the density in the image of a small detail of the subject depends on the size of the detail is taken into account in practical radiography by the use of hole-type penetrameters to measure radiographic sensitivity. In using such a penetrameter, the criterion of radiographic sensitivity is not the visibility of the outline of the penetrameter but the visibility of the smallest hole, for which the diameter is 1 or 2 times the thickness of the penetrameter.

In order to describe the role of the film in the rendition of fine detail, it is convenient to compare the average value of $\Delta \mathrm{D}$ produced by $a$ thickness difference $\Delta t$ with the expression for the range in $D$ as a function of the area.

\section{A. The Average Value of $\triangle \mathrm{D}$}

For a small thickness difference $\Delta t$, the average value of $\Delta E$ is given by

$$
\Delta E=\mu E \Delta t \quad,
$$

where $\mathrm{E}$ is exposure and $\mu$ is the linear absorption coefficient. To a first approximation,

$$
\mathrm{D}=\mathrm{K}_{1} \mathrm{E} ; \frac{\Delta \mathrm{D}}{\mathrm{D}}=\frac{\Delta \mathrm{E}}{\mathrm{E}}
$$

and

$$
\Delta \mathrm{D}=\mu \mathrm{D} \Delta \mathrm{t}
$$

Thus it is seen that the value of $\Delta \mathrm{D}$ for a given $\Delta \mathrm{t}$ and a given $\mu$ increases linearly with D. Qualitatively, this is in agreement with the practical observation that radiographic contrast increases with the density of the radiograph.

This expression for $\Delta D$ appears often in the literature for the case of no scattered radiation from the subject.

B. The Statistical Fluctuation in D

The developed density of a film uniformly exposed to $\mathrm{X}$ rays has a nonuniformity that is visible to the unaided eye. The individual grains of the developed film are too small to be visible without magnification, so the structure that we see is due to larger-scale variations in the population of the developed grains. 
In the exposing process, the individual crystals of the film, acting independently, absorb energy from other discrete entities - X-ray quanta and electrons - each of which can produce developability in one or more crystals. For a given average exposure, the nonuniformity of the developed image will depend on the nonuniformity in the distribution of the se individual absorption acts over the area of the film.

A simple expression for the magnitude of the statistical fluctuation can be derived from the characteristic curve of the film. Over the range of practical densities, $\mathrm{D}=\mathrm{k}_{1} \mathrm{E}$, where $\mathrm{E}$ is the average exposure over a large area. Now $\mathrm{E}$ is linearly proportional to the number of $\mathrm{X}-\mathrm{r}$ ay quanta per unit area, $\mathrm{n}$, which are effective in producing density. Thus, $\mathrm{E}=\mathrm{k}_{2} \mathrm{n}=$ $\mathrm{k}_{2} \mathrm{~N} / \mathrm{a}$, where $\mathrm{N}$ is the number of effective quanta in the area a. It follows that $\mathrm{D}=\mathrm{k}_{1} \mathrm{k}_{2} \mathrm{n}$, or $\mathrm{D}=\mathrm{kn}=\mathrm{kN} / \mathrm{a}$.

The value of $\mathrm{k}$ is a constant, characteristic of the particular film. Faster films will require fewer effective quanta per unit area to produce a given density, so $\mathrm{k}$ increases with film speed. The value of $\mathrm{k}$ will also depend on other factors, such as the average quantum energy and the type of intensifying screens. Encompassing all of these factors, we can state that $\mathrm{k}$ is a measure of the speed of the radiographic system, measured in terms of the number of X-ray quanta required to interact with the system to produce a given density. The absolute value of $k$ is not easy to determine in most cases, but for present purposes we do not need to know it.

Note, however, that $\mathrm{n}$ is not the number of quanta incident on the film-screen combination, nor the number absorbed by the film-screen combination. It is the number of quanta which interact with the radiographic system in any way that results in the production of density on the film. All other quanta entering the system have no effect on density or the appearance of the radiograph.

Since $\mathrm{N}$ represents a number of random, independent events, the fluctuation in $\mathrm{N}$ can be expressed as the standard deviation

$$
\sigma(N)=\sqrt{N},
$$

or

$$
\sigma(N) / N=1 / \sqrt{N}
$$

Then, since $\mathrm{D}=\mathrm{kN} / \mathrm{a}$,

$$
\begin{aligned}
\sigma(D) / D & =\sigma(N) / N \\
\sigma(D) / D & =1 / \sqrt{N}=\sqrt{k / D a} ; \\
\sigma(D) & =\sqrt{D k / a} .
\end{aligned}
$$


It is seen that the fluctuation $\sigma(D)$ in $D$ is greater for small values of a, and hence for the small-scale features of the subject being radiographed. Also, $\sigma(D)$ increases with the square root of the density and with the square root of $\mathrm{k}$, so that if all other conditions of exposure are constant, $\sigma(D)$ will increase as the square root of film speed.

To test the validity of this treatment in terms of practical significance, we can consider a penetrameter with a hole of area a and depth $\Delta t$. The density difference between the radiographic image of the hole and its immediate surroundings will be given by $\Delta \mathrm{D} \pm \sigma(\mathrm{D})$, and $a$ measure of the visibility of the image will be given by the ratio*

$$
\Delta \mathrm{D} / \sigma(\mathrm{D})=\mu \mathrm{D} \Delta \mathrm{t} / \sqrt{\mathrm{Dk} / \mathrm{a}}=\mu \Delta \mathrm{t} \sqrt{\mathrm{Da} / \mathrm{k}} .
$$

For a particular subject and kilovoltage (fixed $\mu$ ), the visibility of the hole should increase with hole depth as the square root of the density on the developed film and the area of the hole, and should vary inversely as the square root of film speed, if the quantum nature of the exposing radiation has an important effect on the visibility of fine detail in the radiographic image. The next section presents a recent experiment designed to study this problem.

C. Experimental Study of the Rendition of Small Holes

\section{Experimental Procedure}

Figure 5 is a drawing of a one-inch-thick plate of steel containing holes of various diameters and depths which was radiographed at $200 \mathrm{KVP}$ with fixed geometry and with various films and at various densities. Three replicate radiographs were made for each set of conditions, and each radiograph was examined by 3 observers to determine for which holes the images were just visible. A summary of the test plan is given in Table I.

* It can be shown that

$$
\Delta \mathrm{D} / \sigma(\mathrm{D})=\Delta \mathrm{T} / \sigma(\mathrm{T})
$$

Rose(4) has found that for just-visible images

$$
\Delta \mathrm{T} / \sigma(\mathrm{T})=5 .
$$




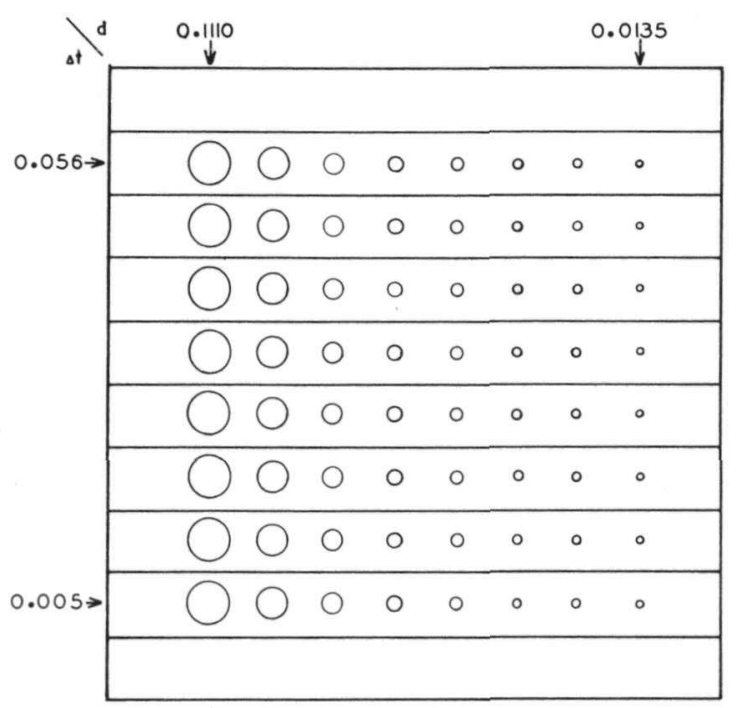

Fig. 5

Schematic diagram of the 1 -in.-thick test plate. In each horizontal row the depth is constant and in each vertical column the diameter is constant. The maximum and minimum diameters and depths are as indicated.

TABLE I

SUMMARY OF THE TEST PLAN FOR THE

STEEL PLATE WITH 200-KVP X RAYS

\begin{tabular}{|c|c|c|c|}
\hline Test Series & Variable & Film & D \\
\hline 1 & Film & A, B, C, F, & 1.6 \\
2 & D & A & $0.9,1.3,1.6,2.0$ \\
\hline
\end{tabular}

All exposures were made at a source-to-film distance of 51 in., with the film in a vacuum cassette with 0.005-in. lead screens (the same cassette and screens for all exposures). The cassette was backed with a large sheet of lead, $\frac{1}{4}$ in. thick, to minimize back scatter from the surroundings.

The exposed films were processed manually according to the recommended times in a commercial X-ray developer at $68^{\circ} \mathrm{F}$ with carefully controlled agitation in both developer and acid short-stop.

Examination of the radiographs was carried out in a room with a low level of illumination and with a mask over the radiograph. The mask was arranged so that the examiner could move a $\frac{1}{2}$-in.-diameter aperture along a single row of images for holes of the same depth. Proceeding from the smallest diameter toward the largest, the observer was asked to determine the first visible hole image in the row. 


\section{Results}

If the theory presented above is correct, $\Delta D / \sigma(D)$ should be equal for images which are equally visible. Thus, for the just-visible images,

$$
\mu \Delta t \sqrt{\mathrm{Da} / \mathrm{k}}=\text { Constant }=\mathrm{C} \quad .
$$

Expressing the area a in terms of the hole diameter $d, \sqrt{a}=\sqrt{\pi} d / 2$, there is found

$$
\log \Delta t=\log \frac{2 c}{\mu \sqrt{\pi}}-\log \sqrt{D}+\log \sqrt{k}-\log d \quad .
$$

A plot of log $\Delta t$ versus log d of the just-visible holes for constant $\mu$ and $\mathrm{D}$ should give a straight line of slope -1 for each film, and the lateral displacement along the axis of log d of the lines for 2 films (different values for $k$ ) should be equal to the log of the square root of the relative film speeds, as given by the relative exposure times required to produce the radiographs.

Figure 6 presents the data for the 4 films at $200 \mathrm{KVP}$ and $\mathrm{D}=1.6$. It is seen that the straight lines of slope -1 represent the data within the experimental error of the experiments as represented by the scatter of the points.

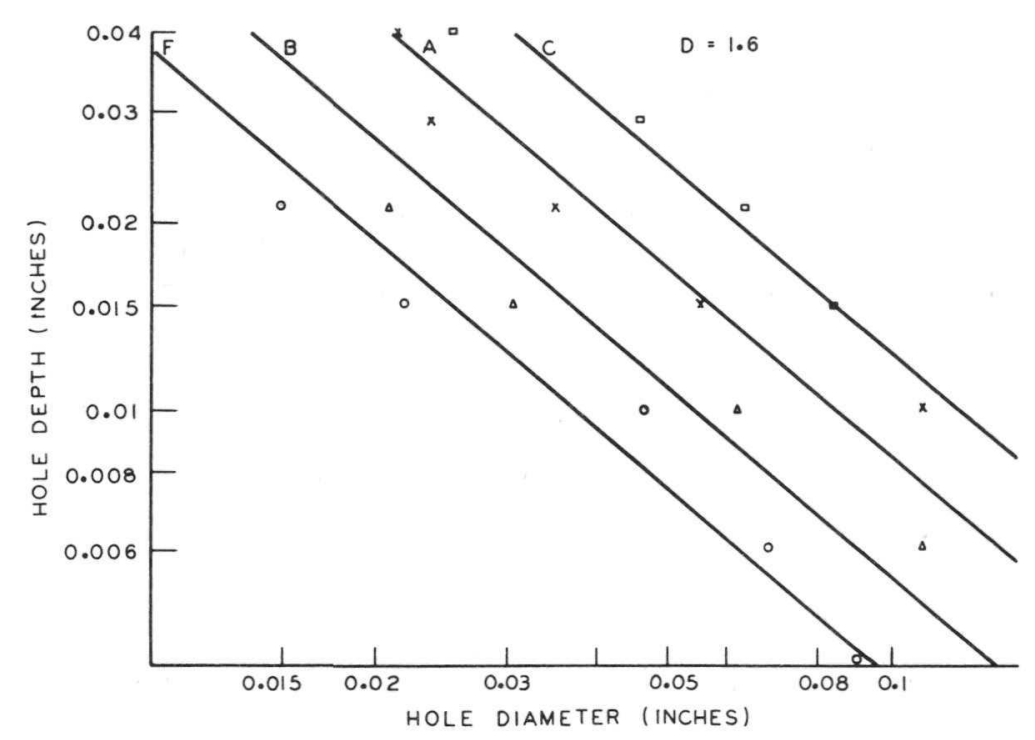

Fig. 6. Relationship between depth and diameter of the holes of the test plate for which the images are just visible. The straight lines of slope -1 are based on the theory that statistical fluctuations in the radiation affect fine detail rendition. The points represent experimental data for four films. 
The lines for the various films are displaced, as expected, according to the relative speeds of the films. The line for the slowest film is on the left; the lines for the other films are in the order of increasing speed toward the right, indicating that for holes of a given depth the diameter of the smallest hole for which the image is visible increases with film speed. Calculations show that the separations of the lines are approximately equal to the square root of the relative film speeds, as the theory indicates, but additional experiments will be necessary to check this aspect accurately.

In the second series of tests, film $A$ was exposed to produce various densities. From the expression for log $\Delta t$, a plot of lot $\Delta t$ versus $\log \mathrm{d}$ for just visible holes should yield straight lines of slope -1 and lateral displacements equal to the differences in log D. Figure 7 presents the data for this experiment. Again, the straight lines of slope - 1 represent the data within the experimental error. As expected, for holes of a given depth the diameter of the smallest hole for which the image is visible decreases as the density increases. The separations of the lines as drawn are only approximately in the ratio of the square root of the densities; additional experiments will be necessary to establish the positions of the lines more accurately.

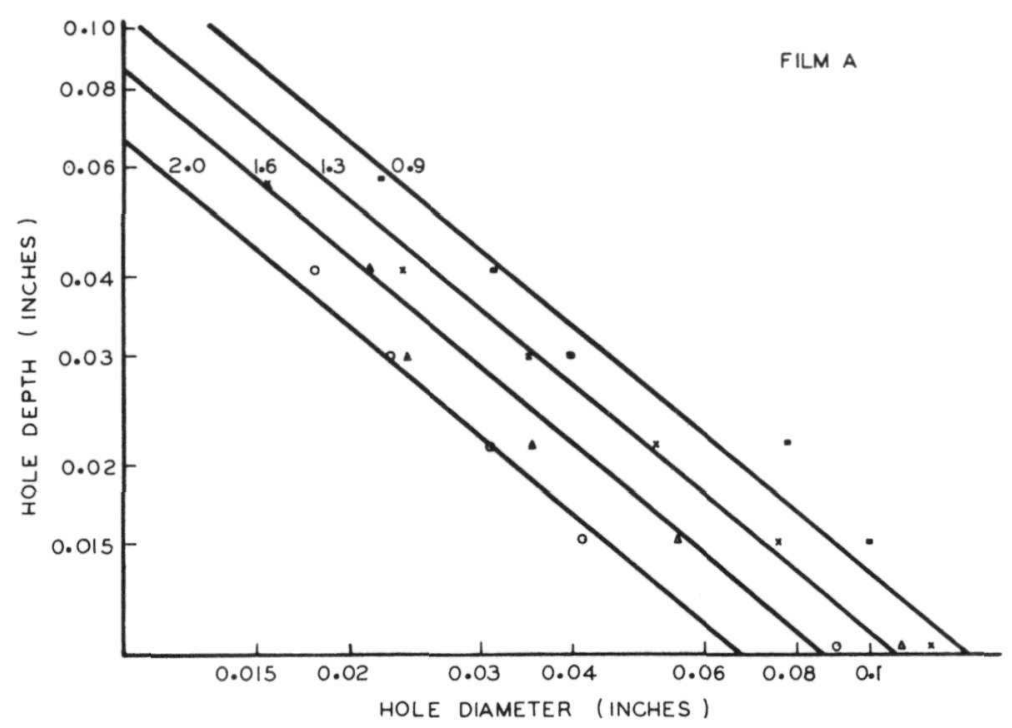

Fig. 7. Relationship between film density for film A and the depth-diameter relationship for justvisible images of holes in the test plate. The straight lines of slope -1 are based on the theory; the points are experimental data for the four density levels indicated. 
3. Conclusions

The experimental results presented above are consistent with the theory that the ability of a radiographic system to depict small-scale detail may be materially influenced by the statistical fluctuations arising from the quantum nature of the exposing radiation. Similar results have been reported in the literature for medical radiography, (1) exposure of film to electrons, (2) and, to some extent, for industrial radiography. (3) In general, however, the effects of the quantum fluctuations have not been taken into account in the literature wherein the characteristic curve is used to derive expressions for radiographic sensitivity. In those cases, the results derived cannot be applied to rendition of fine detail or to penetrameter sensitivity as measured with hole penetrameters.

\section{Discussion}

We have purposely approached the subject of the characteristic curve in a general way in order to emphasize the fundamental difference between the rendition of large-scale and small-scale features of the subject. The technique charts used by radiographers are actually transformations of the characteristic curves of the film as modified by the absorption characteristics of the subject. They are used by the radiographer to predict the rendition of large-scale features. But for the prediction of the rendition of small-scale detail the radiographer resorts to the use of penetrameters with holes.

Although we have found, on a theoretical basis, that statistical fluctuations in the exposing radiation may have a material effect on the rendition of fine detail, it is recognized that other factors may also enter and, in some cases, be predominant. Specifically, we refer to the geometrical relationships of the source, subject, and film and to the effects of scattered radiation. These are not influenced by the characteristics of the film and hence are beyond the scope of this paper. One other factor of this type which is discussed in the literature as "inherent unsharpness of the film" may also be important, although the determination of this property is subject to many experimental problems. At any rate, it appears to us that any theoretical evaluation of radiographic sensitivity should include a consideration of the effects due to the statistical fluctuation in the exposing radiation.

\section{Acknowledgment}

It is a pleasure to acknowledge the assistance of $\mathrm{Mr} . \mathrm{H} . \mathrm{S}$. deBen and $\mathrm{Mr}$. D. P. Jones in the preparation of this paper. 
References

1. Sturm, Ralph E., and Russell H. Morgan, Am. J. Roentgenology and Radium Therapy, 62, 617 (1949)。

2. Frieser, Von H., and E. Klein, Mitteilungen aus den Forschungs laboratorien der Agfa Leverkusen-Munchen, II, 121 (1958).

3. Nondestructive Testing Handbook, Edited by Robert C. McMaster, The Ronald Press Company, New York (1956), Vol. 1, p. 19.28.

4. Rose, A. J., Opt. Soc. Am., 38, 196 (1948).

\title{
Discussion
}

\section{MEANING OF THE CHARACTERISTIC CURVES}

\begin{abstract}
L. A. Turner, Argonne National Laboratory: I think Mr. Larson has given a most convincing talk to demonstrate that these statistical effects are indeed important. There's one aspect that would be interesting from a purely scientific point of view, but I think it also has its practical side in connection with the theme of this conference: that is, the question of how such results might depend on the shape of the hole. The experimental uncertainties extend to the place where you have the psychology of an observer, and obviously what the observer is doing is some way or other making a judgment of the fluctuations of the density in the region of the hole against the densities of the surrounding regions. When he can't see any essential difference, then there isn't any hole. It's quite conceivable that the results could be different, say, for square and circular holes, and perhaps more importantly for long, thin holes. The latter would have a bearing on the matter of how well one can detect cracks in samples. Now, to be sure, one wouldn't expect the general results to be any different. The dependence on density and on film speed would be the same, but the judgment of observers might have something to do with the sensitivity of detection of defects of different kinds.
\end{abstract}

E. T. Larson: You are right, and this is one of the reasons why we used the penetrameter illustration. A penetrameter, the edges of which are essentially an elongated linear nonuniformity - a crack, will oftentimes be visible under a particular technique whereas the circular holes will not. The question of how we see these patterns is still a subject for research. But I think that just intuitively one could say that if a crack is long enough, long enough to be called a crack or otherwise, it would probably be called a hole; it will provide the eye with many opportunities to compare density on one side with the other. So we could simulate the visibility of the crack by putting a lot of holes in a row, and then, I think, you could easily see that you're ironing out the statistics in the process. You have a lot of 
chances then. It is quite true that the visibility of a particular defect will depend on its shape; we have chosen the circular shape in order to make the presentation clear and to simulate the practical case of the penetrameter.

P. D. Edwards, Los Alamos Scientific Laboratory: I seem to have missed a connective link in your theoretical work versus your experimental work. You derived your theoretical work on the basis of statistical variation, and, nevertheless, your experiment was based upon the observation of a single radiograph of a series of holes. Consequently, the question in my mind is: if you took that same radiograph for, say, a hundred times, wouldn't you get the statistical variation in your results even with the same observers?

E. T. Larson: The experiment was based on the observation of 3 different radiographs and not 3 copies of the same radiograph. We had 3 people looking at 3 different radiographs, and so we had 9 values. Generally speaking, we found that when we took the care which we did, all 9 values sometimes centered on the same hole. Sometimes there would be a variation on one side or the other but, by and large, there were not very wide variations. Now, I suspect that some of this fluctuation that you see may be related to the shape of the hole. It was assumed to be circular, but it may not have been circular. Perhaps we don't know the size of the hole as accurately as we think we do, and that may be another reason for some of the scatter that you see there. Now let me explain what we did. Perhaps it was not made clear in the course of the discussion. We say that $\triangle D / \sigma(D)$ is a measure of the visibility of a hole. Then, we find by a sort of null method which holes are just visible, and then their visibility, by definition, is the same. So the ratio $\Delta D / \sigma(D)$ must be the same for those holes, that is, $\Delta D / \sigma(D)=C$. When the results are plotted in the way which we used, one would expect that the points would fall on the straight lines which we drew. Now the reason for doing this was to show that, based on this theory, the practical radiographer can obtain different degrees of image quality by changing the film and similarly by changing the density.

L.A. Turner: Coming back to the point that was just made - I think I'm jumping to hasty conclusions - but I would say that perhaps with even just the one exposure, assuming that it is one plate, one should still follow your line of arguing. It goes this way. The observer is aware, or at least takes in, even if unconsciously, this pseudo-graininess, or mottling, or statistical variation, or whatever you want to call it, and the question that he has to face is whether there is a region inside of this half-inch hole where there is an apparent deviation from what is the natural pseudograininess. You may have overexposure or underexposure because of this statistical variation, but the question is still: is it abnormally high or low? If it's abnormally high, then one can say there is a hole. 


\section{E. T. Larson: I agree with you.}

N. S. Beyer, Argonne National Laboratory: Early in your talk you commented that the general shape of the characteristic curves is the same for different energies. When are the curves not generally the same? What are your high- and low-energy limits?

E. T. Larson: I should have restricted it specifically from 50 KVP on a regular industrial X-ray unit up to cobalt-60 gamma rays, which is as high up as we have ever looked ourselves. Now, there are some slight variations which are of importance if one is considering such matters as the mechanism of latent image formation, but from a practical standpoint they're not of any importance.

D. S..Eppelsheimer, Missouri School of Mines: Was this film doubleemulsion film or single-emulsion?

E. T. Larson: It was double-emulsion film.

D. S. Eppelsheimer: From the layman's point of view, can one generalize your comments by saying that you've determined the resolving power of the emulsion?

E. T. Larson: The layman might use the terminology, but in the photographic industry that has a very specific meaning referring to the ability to photograph a picket fence, if you will. I would say the best thing would be to use an operational type of description. The penetrameter sensitivity for hole-type penetrameters depends on the fact that the X-ray exposure is a quantum process; density is achieved as a result of a finite number of individual random acts which are subject to the laws of statistics. If these are taken into account, one can rather well account for the different abilities of films to show these holes. 
FACTORS AFFECTING RADIOGRAPHIC SENSITIVITY

by

\author{
William D. Kiehle \\ Industrial X-Ray Sales \\ Eastman Kodak Company \\ Rochester, New York
}

\begin{abstract}
This lecture discusses and illustrates the various points affecting radiographic sensitivity, namely, radiographic contrast and definition. Under the main heading "Radiographic Contrasts," the speaker will discuss subject contrast and film contrast, and the factors affecting each. Under the main heading "Definition," there are two definite points of discussion - geometric factors and graininess factors. These will be discussed in detail with illustrations showing how definition, and eventually radiographic sensitivity, may be affected by changing each of the subdivisions of these various factors.
\end{abstract}

The one point that we don't want you to miss is that "Quality is our Objective," not only in product, but also in obtaining the best "Radiographic Sensitivity." Why do we use radiography in industry? - in order to examine the internal conditions of a specimen without destroying it or damaging it. It is important that the maximum amount of information is made available to the inspector. How do we do this? The first prerequisite is the proper radiographic equipment; the second, the best recording film; and the third, the proper technique to give maximum radiographic sensitivity. It is this last factor - radiographic sensitivity - that I will discuss today.

Before we progress any further, we should define a radiograph. A radiograph is a photographic record produced by the passage of $\mathrm{X}$ rays or gamma rays through an object onto a film. When film is exposed to $X$ rays, gamma rays, or light, an invisible change is produced in the film emulsion. The areas so exposed become dark when the film is immersed in a developing solution, the amount of darkening depending upon the degree of exposure. For example, if the object is a steel casting having a void formed by a gas bubble, the void results in a reduction of the total thickness of steel to be penetrated. Hence, more radiation will pass through the section containing the void than through the surrounding metal. A dark spot, corresponding to the projected position of the void, will appear on the film when it is developed. Thus, a radiograph is a kind of shadow picture: the darker regions on the film represent the more penetrable parts of the object and the lighter regions the less penetrable. 
What do we mean by radiographic sensitivity, or in other words, quality in a radiograph? Radiographic sensitivity is a general or qualitative term referring to the smallest detail that can be seen in a radiograph or to the ease with which the images of small details can be detected. Phrased differently, it is a reference to the amount of information contained in a radiograph. In order to produce better radiographs, we must have an understanding of the factors that comprise radiographic sensitivity, namely, radiographic contrast and definition. Radiographic contrast is the resultant of both subject contrast and film contrast. Definition is the resultant of geometric factors and film graininess factors.

Radiographic contrast consists of the density differences in the radiograph between one area and another. Any shadow or detail within the image is visible by reason of the contrast between it and its background or surrounding structures. Within appropriate limits, the greater the contrast or density differences in the radiograph, the more definitely various details stand out, and the finer the detail that can be seen. Density is defined by Van der Platts in this way: "When the film emulsion is exposed to $\mathrm{X}$-radiation, the particles of silver bromide absorb energy and so become capable of photographic development. This means that when the film is placed in a reducing agent the energized grains of silver bromide are reduced to free metallic silver deposits, the density of which will be greatest at the parts which have absorbed the greatest amount of energy. Silver is naturally opaque and when finely distributed gives the impression of being black. The transparency of the film will be least, the opacity greatest, in those parts which contain the densest deposit of silver; that is, those which received the greatest exposure." Density, in other words, is the amount of developed silver in any area of a radiograph. It is the "light-stopping power" of that area and is measured by a densitometer. The figures we use to express density - those on the scale of the densitometer - are derived like this:

$$
\text { TRANSMISSION }=\frac{\text { Amount of light that gets through any area }}{\text { Total amount of light striking that area }} .
$$

It is usually denoted by "T" and is expressed as a percent, so you have to multiply the above fraction by 100 . A transmission of 85 percent means that 85 percent of the light that strikes any specific part of a negative or transparency gets through it.

$$
\text { OPACITY }=\frac{\text { Total amount of light that strikes any area }}{\text { Amount of light that gets through that area }}
$$

As you can see, it is the transmission turned upside down. So opacity $\mathrm{O}=1 / \mathrm{T}$. We don't use percent here; so, for our above example $(\mathrm{T}=85 \%)$, the opacity (O) is $1 / 0.85$ or 1.175. Now, remember the higher the opacity, the less light gets through. 
Now, density D relates directly to the opacity; it is the logarithm of the opacity (to the base 10):

$$
\begin{aligned}
& \mathrm{D}=\log \mathrm{O}(\text { or } \mathrm{D}=\log 1 / \mathrm{T}) \text { or, as in the example, } \\
& \mathrm{D}=\log 1.175 \text { or } \mathrm{D}=0.07
\end{aligned}
$$

For instance, if we allow 100 units of light to fall on the surface of a film and 100 percent of this light is transmitted, we would say that its opacity is 100 divided by 100 or 1 . In this case, obviously, we did not have any density on the film. Now, suppose we take a second film and direct 100 units of light onto its surface. If 50 percent of this light passes through the film, we would than have an opacity of 2 (obtained by dividing 100 incident-light units by 50 transmitted-light units). The logarithm of 2 is 0.3 , so this film has a density of 0.3. In our third film, suppose that with 100 units of light incident on the film, we have $12 \frac{1}{2}$ percent transmitted. Then 100 divided by $12 \frac{1}{2}$ equals an opacity of 8 and the logarithm of 8 is 0.9 . Thus, the density is 0.9. The density in any part of a negative, with any film-development combination, depends on two things: first, the exposure which that part of the radiograph received; second, the amount of development given the film.

Let's draw a picture of that relationship. To do it, we make a series of exposures on a sheet of film (like a test strip) and develop it. Then

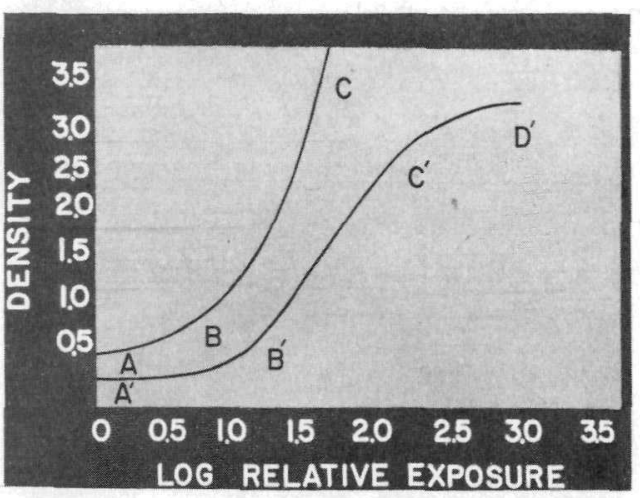

Fig. 1. A Typical Characteristic Curve we'll make a graph by reading the densities in the developed strip and plotting them against the logarithms of the exposures that we used to produce them. (We use the logarithms of the exposures because the density is logarithmic.) Figure 1 is the characteristic curve, or $\mathrm{H}$ \& D curve (after Hurter and Driffield, the men who devised it), or D-log E curve. We'll call it the "characteristic curve" of a film. This curve actually describes the photographic characteristics of the film for the development given. Densities falling on the "toe" portion (A-B) of our curve are not directly proportional to the log expo-

sures. Neither are the "shoulder" (C-D) densities. That doesn't mean that we shouldn't use these two parts of the curve. We do. The main thing here is to know what the "toe," "shoulder," and "straight-line" portions of the curve are, and what they mean to use in our radiographic procedures. The section B-C is the "straight-line" portion of the curve; the density in the film increases proportionally with the log of the exposure. The slope of this straight-line portion of this curve is said to be film contrast: the steeper the slope, the higher the contrast. 
Because density and film contrast are so closely tied together, I would like to put the cart before the horse and discuss film contrast before subject contrast. The first item of discussion under film contrast is the type of film used. Because film contrast is built into the film when it is manufactured, it may vary with the type of film, the processing the film receives, and the density at which the slope is measured.

Film contrast can be changed by using different development procedures. To illustrate this point, we took three strips of one type of film and made a stepped-wedge exposure on each, using the same kilovoltage and exposure. Then, we developed one for $2 \mathrm{~min}$, another for $5 \mathrm{~min}$, and the third for $8 \mathrm{~min}$. Figure 2 readily shows change in the contrast of the radiographs. This can be shown graphically by their characteristic curves. Note how the slope changes with change in development. Figure 3 illustrates the $\mathrm{H} \& \mathrm{D}$ characteristic curves of an industrial X-ray film developed at three different times: $2 \mathrm{~min}, 5 \mathrm{~min}$, and $8 \mathrm{~min}$. And you can see that as the development time is increased, the contrast of the film or the slope of the curve increases. The 2 -min developing time yields the curve of lowest slope and therefore having lowest film contrast; the 5-min film is intermediate and the 8 -min development time film is the one with the highest contrast. I feel I should point out here that increasing the development time to about $8 \mathrm{~min}$ does not necessarily mean that you will increase the contrast continuously. You will reach a point at which an increase in development time will cause an increase in base fog, or developer fog, which in turn will reduce the inherent film contrast.

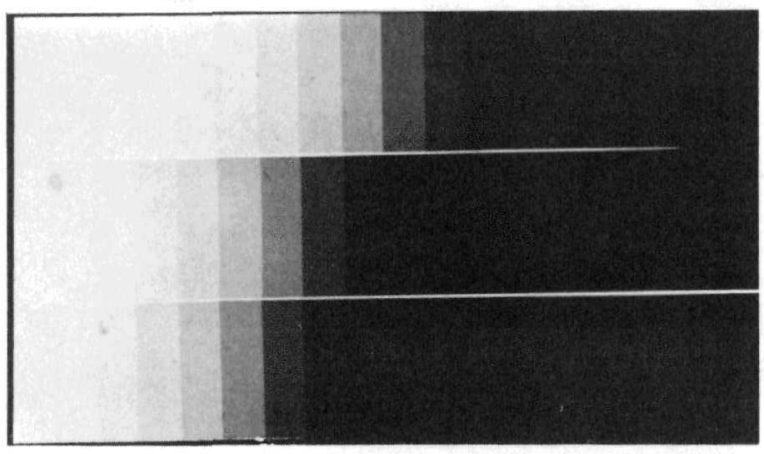

Fig. 2. Density Step Wedges

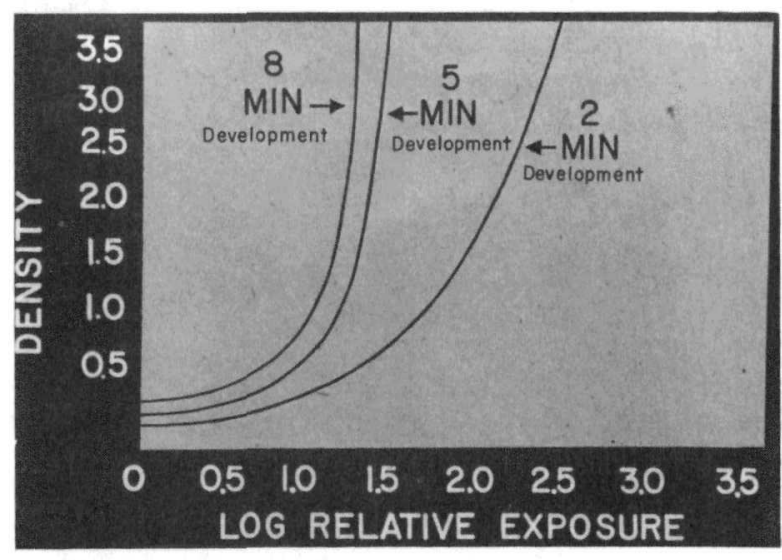

Fig. 3. Characteristic Curves: Development Times

As I mentioned before, film contrast will also vary with density. In industrial radiography, where film such as Type $M$, Type AA, and Type KK have high maximum density, the contrast of the film increases with density. The Kodak Industrial X-ray Film, Type F, reaches its maximum gradient at a density of about 2.0 and then tends to fall off, whereas 
the Type KK, the No-Screen, the Type AA, and the Type M increase in gradient or contrast as the density increases. Therefore, exposure of your films to higher densities in your radiographic inspection when using Types M, AA, No-Screen, or KK will give you a higher radiographic contrast in the final radiograph.

In practice, subject contrast must be given first consideration in order to choose a film type that will produce a radiograph of optimum "Radiographic Contrast." The first factor in subject contrast is, of course, the specimen itself - its variations in thickness, its physical density, and its atomic number. Figure 4 illustrates what is meant by subject contrast. This is the radiograph of three different "C" clamps of varying thickness, using Type AAFilm. The smallest "C" clamp illustrates low subject contrast and the largest, high subject contrast. The second factor which affects subject contrast is kilovoltage or the quality of radiation used to make the radiograph. Low kilovoltage produces long-wavelength X rays high kilovoltage produces short-wavelength $\mathrm{X}$ rays. The penetrating power of the longer wavelength is not as great as that of the rays at higher kilovoltage. Therefore, the higher the energy, the lower the subject contrast and vice versa. The third factor that affects subject contrast is scattered radiation. Scattered radiation is one of the most important and one of the least-understood factors affecting radiographic contrast. For this reason, let us give it more than passing attention.

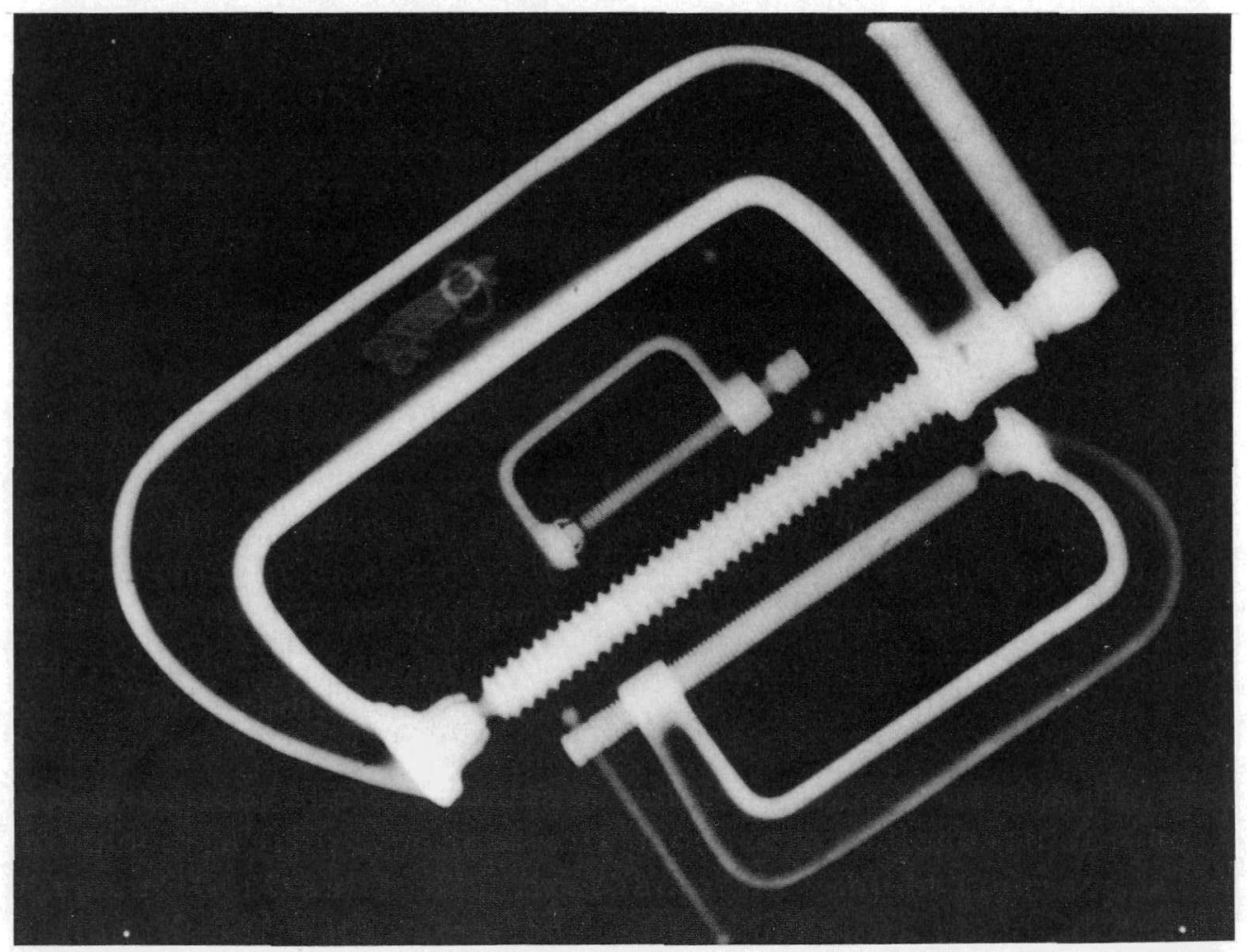

Fig. 4. Radiograph of "C" Clamps 
When a beam of $\mathrm{X}$ rays or gamma rays strikes any object, some of the rays are absorbed, some are scattered, and some pass through. Electrons of the atoms comprising the object scatter radiation in all directions, much as light is dispersed in a fog. The wavelengths of much of the radiation are increased by the scattering process, and, hence, the scatter is always somewhat "softer" or less penetrating than the unscattered primary radiation. Any material, whether specimen, cassette, table top, walls, or floor, that receives the direct radiation is a source of scattered radiation. It is non-image forming radiation, and, therefore, objectionable. Unless suitable measures are taken to reduce the effects of scatter, it will cause a haziness or fog over the whole or part of the radiograph, thus reducing contrast. The effect of scattered radiation is not due to some peculiarity of scattered rays as compared with primary rays. It simply changes the relative intensities coming through the specimen regardless of the film used. The following table shows how the subject contrast is affected by scattered radiation. The values are assumed but are known to be reasonable.

\begin{tabular}{|c|c|c|c|}
\hline $\begin{array}{l}\text { Primary } \\
\text { Intensity } \\
\text { through Flaw }\end{array}$ & $\begin{array}{l}\text { Primary Inten- } \\
\text { sity through } \\
\text { Surroundings }\end{array}$ & $\begin{array}{l}\text { Secondary } \\
\text { Intensity }\end{array}$ & Contrast \\
\hline 5 & 1 & 0 & $\frac{5+0}{1+0}=5$ \\
\hline 5 & 1 & 1 & $\frac{5+1}{1+1}=3$ \\
\hline 5 & 1 & 2 & $\frac{5+2}{1+2}=2 \frac{1}{3}$ \\
\hline 5 & 1 & 3 & $\frac{5+3}{1+3}=2$ \\
\hline 5 & 1 & 4 & $\frac{5+4}{1+4}=1 \frac{4}{5}$ \\
\hline
\end{tabular}

This chart is self-explanatory. Assuming that our primary intensity through the flaw is a constant with a value of 5 and the primary intensity through the surroundings is a constant with a value of 1 , our subject contrast will only change as the amount of scattered radiation or secondary radiation increases or is present. To do this numerically, we go to the fourth column headed by the word "Contrast." With no secondary radiation, we have a subject contrast of $(5+0) /(1+0)$ or 5 . However, if our subject secondary intensity or secondary radiation has a figure of 2 compared with the other primary intensities, then we have a contrast of $(5+2) /(1+2)$ or $2 \frac{1}{3}$, and so on down to the last set of figures in which our secondary radiation has a figure of 4 compared with the other primary intensities and we have a subject contrast of $(5+4) /(1+4)=1 \frac{4}{5}$. So, as you can see very easily, as the secondary radiation tends to increase, our subject contrast tends to decrease. 
In radiography of thick materials, scattered rays form the greater percentage of the total radiation incident upon the film. For example, in the radiography of a $\frac{3}{4}-i n$. thickness of steel, the scattered radiation from the specimen is almost twice as intense as the primary radiation reaching the film. In the radiography of a 2 -in. thickness of aluminum, the scattered radiation is $2 \frac{1}{2}$ times as great as the primary radiation. As may be expected, if this scattered radiation is prevented from reaching the film, a marked improvement is obtained in the quality of the radiographic image. As a rule, the greater portion of the scattered radiation affecting the film arises in the specimen. Primary radiation striking the film holder or cassette directly or through a thin portion of the specimen will also cause scattering into the adjacent thicker portions. Such scatter is called undercut. Another source of scatter which may undercut a specimen is reflection scatter from adjacent specimens. Other materials, such as a wall or floor, on the film side of the specimen may produce scattered radiation. This is called backscattered radiation.

Although scattered radiation can never be completely eliminated, a number of means are available for reducing its effect. A good method is by the use of cutout diaphragms or some other form of mask mounted over or around the object. Where a cutout diaphragm would not be economical, packing barium clay around the specimen will serve the same purpose.

Sometimes it may be found practicable to place the objects in aluminum or thin sheet-iron pans and to use a liquid absorber. A combined saturated solution of lead acetate and lead nitrate is satisfactory, but requires caution in use because it is poisonous. Carbon tetrachloride has also been used as a masking agent for aluminum, but personnel should guard against excessive exposure to its fumes. A convenient, effective arrangement is to surround the object with copper or steel shot having a diameter of about 0.01 in. or less. This material "flows" without running badly. It is also useful for filling cavities in irregular objects, such as castings, where a normal exposure for thick parts would result in overexposure for thinner parts.

Since scatter may also arise from the specimen itself, it is good practice, wherever possible, to use a cone or diaphragm at the tube to limit the cross section of an X-ray beam. A simple metallic filter mounted in the X-ray beam near the tube may be helpful to reduce scatter and to eliminate overexposure in the thin regions of the specimen. The filter material causes a much greater change in the amount of radiation passing through thin parts than through thicker parts. Such a filter is particularly useful in cases in which a mask around the specimen is impractical or in which the specimen would be injured by chemicals or shot. Of course, an increase in exposure or kilovoltage will be required to compensate for the additional absorption; but, in cases where the filter method is applicable, this is not serious unless the limit of the X-ray machine has been reached. In medical radiography, scattered radiation is very effectively removed by the Potter-Buckey diaphragm. This device does not lend itself well to 
industrial procedures because (1) it is not very rugged, (2) it is not as effective for the harder radiations, and (3) it increases the specimen-tofilm distance. However, it is being considered in the radiographic examination of beryllium specimens.

Lead-foil screens, mounted in contact with the film, are beyond a doubt the cheapest, most convenient, and most universally applicable means of combatting the effects of scattered radiation. They lessen the effect of scattered radiation by:

(1) intensifying the primary rays more than the secondary rays, and by

(2) absorbing the secondary more than the primary rays.

As a result, a radiograph made with lead screens usually has more contrast than one made by direct exposure. In radiography of small castings, the principal value of lead screens is in reducing "halation" or scatter undercutting, because the screens will absorb much of the undercut radiation. Lead screens lessen the scatter reaching the film regardless of whether the screens permit a decrease or require an increase in the exposure.

To sum up so far, we have seen that radiographic contrast is the "product" of two distinct factors: film contrast and subject contrast. Now we will consider for a few minutes the other main factor affecting radiographic sensitivity, namely, definition. As mentioned earlier, definition is the resultant of geometric factors and film-graininess factors. It is the degree of distinctness or clarity of detail in a radiograph. To understand the effect of geometric factors on definition, we must recall that a radiograph is a shadow picture of an object which has been placed in the path of an X-ray or gamma-ray beam. Therefore, the appearance of this picture is influenced by the relative positions of the object and the film and by the direction of the beam. Hence, the elementary principles of shadow formation are important to those making and interpreting radiographs. Since $\mathrm{X}$ and gamma rays obey the common laws of light, their shadow formation may be explained in a simple manner in terms of light. The analogy is not perfect, since all objects are, to a greater or lesser degree, transparent to $\mathrm{X}$ and gamma rays and since scattering presents greater problems in radiography than inoptics.

However, the same geometric laws of shadow formation hold for both visible light and penetrating radiation. Suppose that light from a point falls on a white card, and an opaque object is interposed between the light source and the card. A shadow of the object will be formed on the surface of the card. This shadow cast by the object will naturally show some enlargement because the object is not in contact with the card; the degree of enlargement will vary according to the relative distances of the object from the card and from the light source. Thus, the diameter of the 
shadow is to the diameter of the object as the distance of the light from the card is to the distance of the light from the object. The degree of sharpness of any shadow depends upon the size of the source of light and upon the position of the object between the light and the card. When the source of light is not a point but a small area, the shadows cast are not perfectly sharp because each point in the source of light casts its own shadow of the object, and all of these overlapping shadows produce an ill-defined image called penumbra. The form of the shadow also may differ according to the angle which the object makes with the incident light rays. Such deviations in form are called distortion. The basic principles of shadow formation must be considered to assure sharpness and optimum freedom from distortion. A certain degree of distortion naturally will exist in every radiograph because some parts of the object will always be farther from the film than others. However, by the use of an appropriate distance from focus to film, distortion can be lessened to a point where it will not be objectionable. Now let us apply these geometric principles of shadow formation to radiography.

The $X$ rays should proceed from as small a focal spot as other considerations will allow, for there is a definite relationship between the focal spot size and the definition in the radiograph. The finer the focus of the tube, the finer the detail. When a large-focus tube must be used, long source-film distances will aid in showing detail. The film should be as close as possible to the object being radiographed. The distance between the anode and the material examined should always be as great as is practical. Maximum source-film distances should be used in the radiography of thick materials, to minimize the fact that structures farthest from the film are less sharply recorded than those nearer to it. The central ray should be as nearly perpendicular to the film as possible to preserve spatial relationships. As far as the shape of the specimen will allow, the plane of maximum interest should be parallel to the film. Another factor is that the screen should be in as intimate contact with the film as possible. Poor contact between film and screens can produce unsharpness in the image.

The second factor involved in definition is film graininess. The image on an X-ray film is formed by countless minute silver grains, the individual particles being so small as to be visible only under a microscope. However, these small particles group together in relatively large masses. This "clumping," which is quite visible to the naked eye or with a magnification of only a few diameters, produces the visual impression which is called graininess. This clumping can be illustrated by this series of five figures. Figure 5 is an average negative of a girl's face showing the image clearly. Let's enlarge this photographic image and then take a piece of it and look at it under magnification, such as her right eye (see Fig. 6). You will note that we still have an image and that the graininess is becoming excessive. Let's enlarge it again (see Fig. 7). 
You will note that we are starting to lose the image of her eye and are beginning to see more grain. However, we still see some graininess. Figure 8 still shows some clumping of the grains, but we do also see a few individual grains. This is a magnification of several thousands times. In Fig. 9 we see the individual silver grains in the negative, and we have lost our clumping or graininess. All trace of an image is gone. This is an excellent demonstration of what we mean by the term "film graininess." The radiographs should be referred to as having a certain graininess, not as having a certain grain size. What we see in our final radiograph is the clumping of grains, and, therefore, inherent graininess - we do not see the size of the grains.

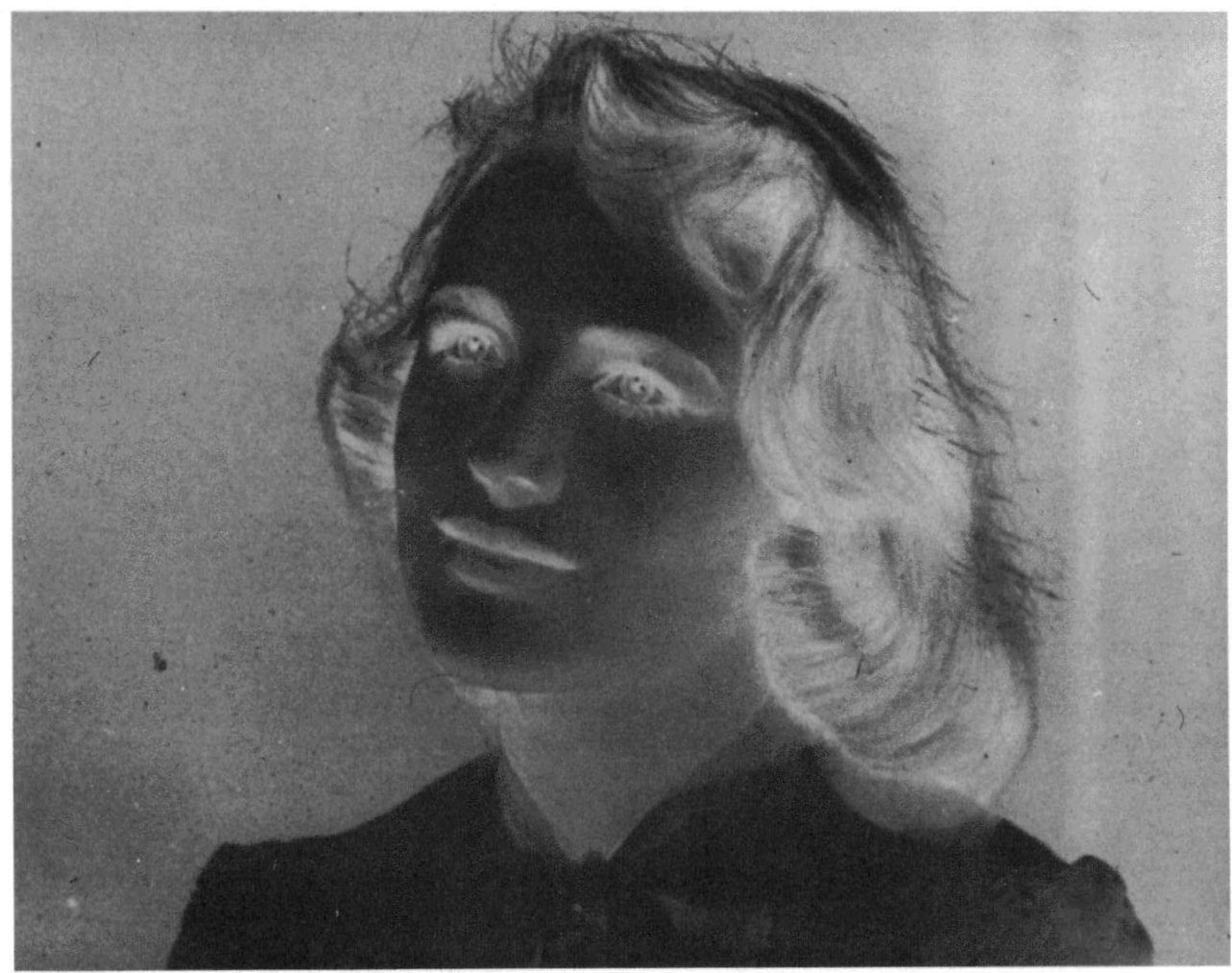

Fig. 5. Negative of a Girl's Face

All films exhibit graininess to a greater or lesser degree. In general. it may be said that the slower films have less graininess than the faster. Thus, Type AA Film is less grainy than Type KK. Also, the graininess of all films increases as the penetration of the radiation increases, although the rate of increase may be different for different films. The graininess of the images produced at high kilovoltages makes the inherently fine-grain films, such as Types AA and Type M, especially useful in the million- and multi-million-volt range. 


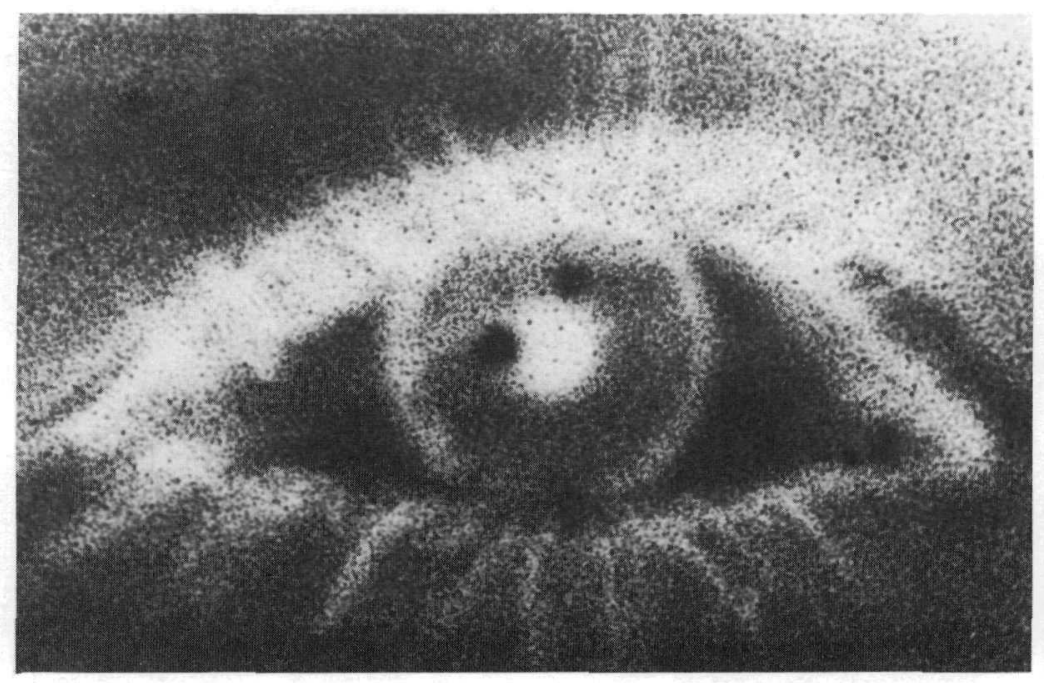

Fig. 6. First Enlargement of Eye

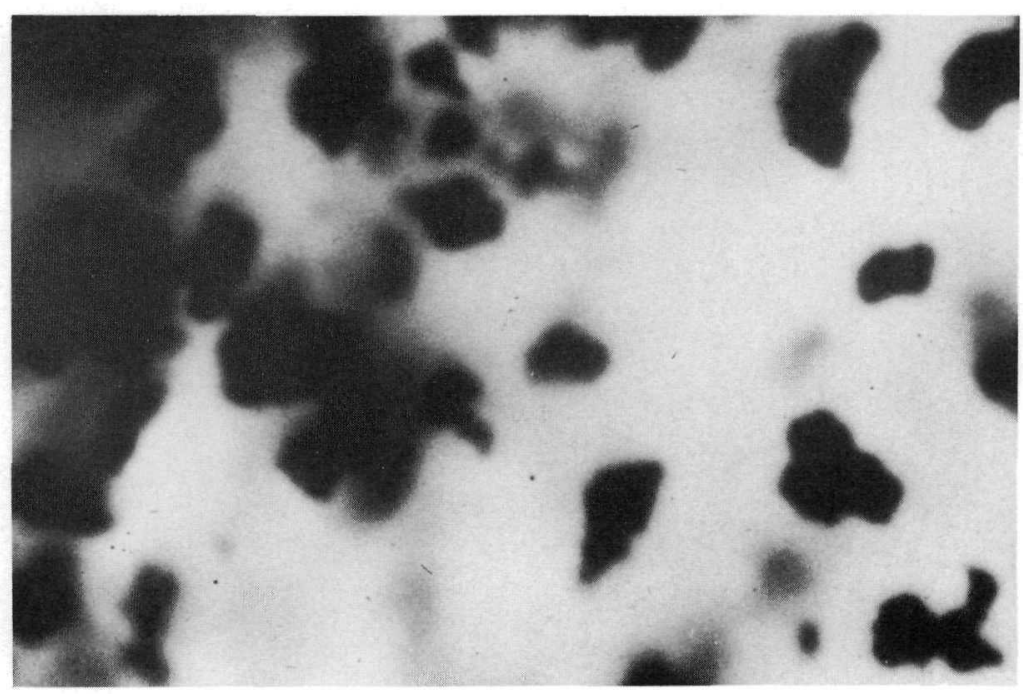

Fig. 8. Third Enlargement of Eye

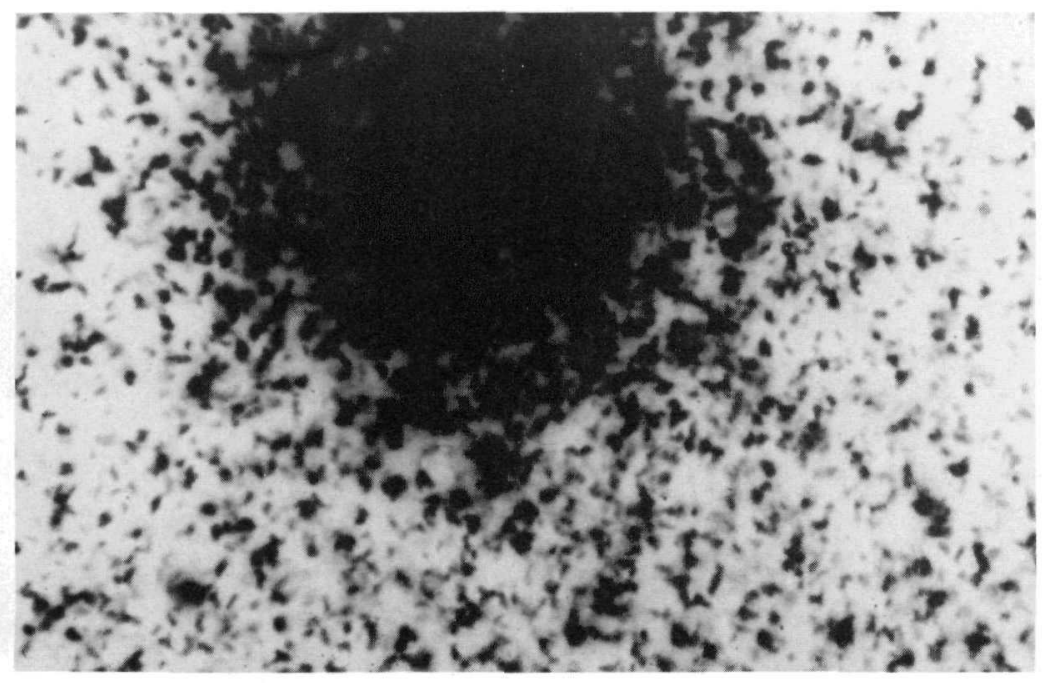

Fig. 7. Second Enlargement of Eye

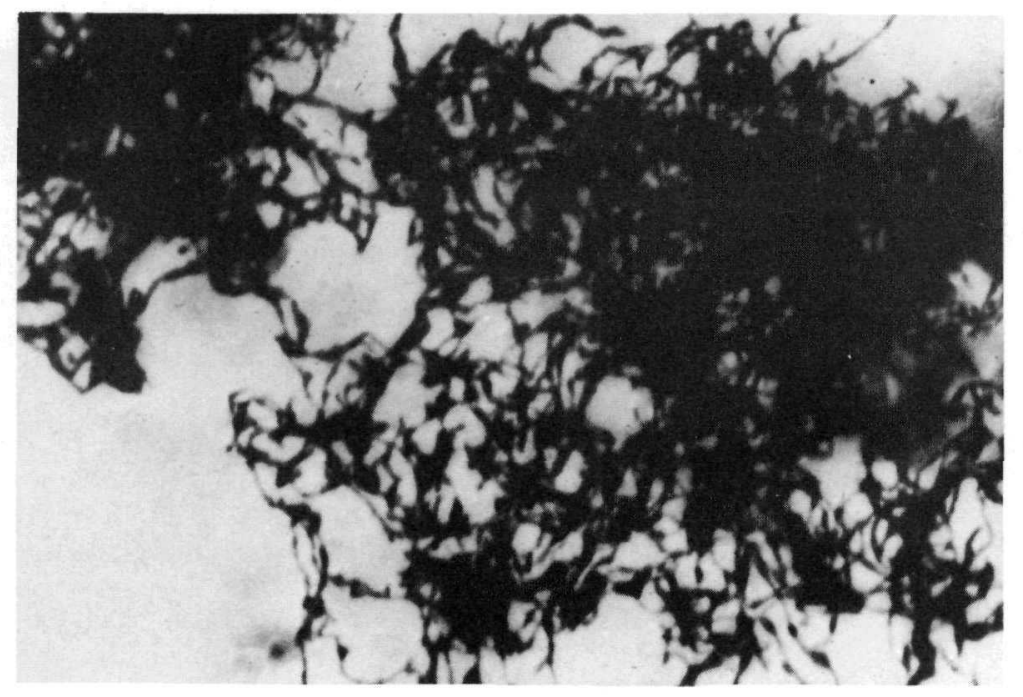

Fig. 9. Final Enlargement of Eye 
This difference in graininess in the various types of industrial films exposed at low and high kilovoltages is readily seen in Fig. 10. Here we see a series of 8 radiographs of a step wedge made with 4 different types of films at 2 different kilovoltages. The types of films are Type KK, Type AA, Type M, Blue Brand X-ray Film with calcium tungstate fluorescent screens, and the two kilovoltages are $40 \mathrm{kv}$ and $1000 \mathrm{kv}$. You can see that the contrast of these radiographs decreases from $40 \mathrm{kv}$ to $1000 \mathrm{kv}$. Also, you can see a change in graininess according to film type, namely, the Type $\mathrm{M}$ in the middle has the finest graininess of all four, whereas Type AA is next, and Type $\mathrm{KK}$ is next. The Blue Brand with calcium tungstate intensifying screens may have the highest contrast of both kilovoltages, but it also has the most graininess.

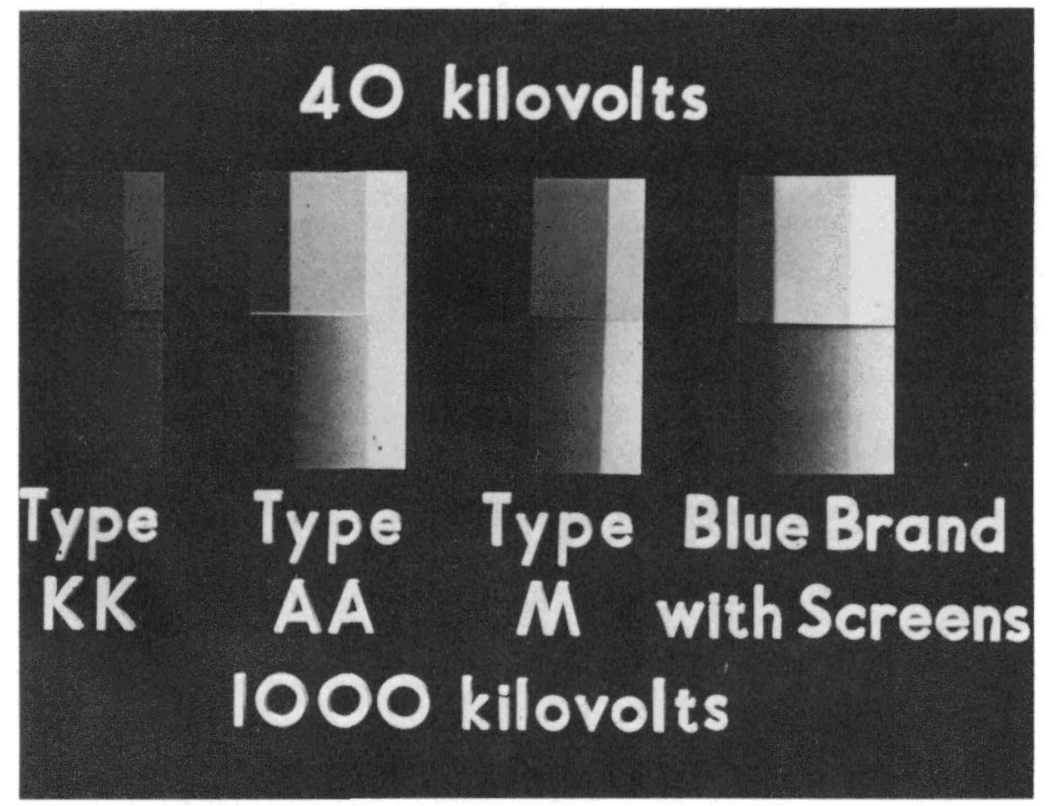

Fig. 10. Radiograph of Step Wedge

Graininess is also affected by processing conditions, being directly related to the degree of development. For instance, if development time is increased for the purpose of increasing film speed, the graininess of the resulting image is likewise increased. Conversely, a developer or developing technique which gives an appreciable decrease in graininess will also result in an appreciable loss in film speed. However, adjustments in development technique made to compensate for changes in temperature or activity of a developer will have little effect on graininess. 
Another source of graininess is encountered in radiography with fluorescent intensifying screens. The graininess of fluorescent screens increases with increased hardness of radiation. The graininess from the fluorescent screens themselves is a fact which limits the use of fluorescent screens at high kilovoltages and with gamma rays. Lead-foil screens have little effect on graininess, although careful tests indicate that an exposure with lead-foil screens has somewhat more graininess than a radiograph made with direct exposure.

Now, in summation, I will say that there are many factors affecting radiographic sensitivity. If each time we made a radiograph it were necessary to run through a check list of all these factors to insure the best work, we would have a system of nondestructive testing which would be extremely cumbersome. Fortunately, however, only a few of these factors need your attention. The first factor, radiographic contrast, is the product of subject contrast and film contrast. As far as subject contrast is concerned, obviously you cannot control the thickness of the specimen, so you must accept it and work with it. The second factor - radiation control or kilovoltage - is within your control. The third factor - scattered radiation is also partially within your control. Under film contrast, we have the type of film. The manufacturers make several types of industrial X-ray films. Since film contrast is inherent to the film in manufacture, there is little you can do except to choose the proper film.

The second factor influencing film contrast is development. This is definitely within your control - through time-temperature development, proper agitation, and precise replenishment of the developing solution. The other important factor affecting radiographic sensitivity is definition, which is comprised of geometric factors and graininess factors. The geometric factors which are affected by the focal spot size are not particularly within your control, since the manufacturer of radiographic equipment provides as small a focal spot size as practicable. It is only necessary that you choose a tube which can accomplish the job without overloading. Thickness change within the specimen is not within your control, and you must simply work with it as it is, but you may control screen-film contact by the use of vacuum or rigid cassettes. The type of film is, again, important with respect to graininess factors. This film quality is inherent to film manufacture, and your control is the choice of proper film. The type of screens you use will be dictated by experience and equipment limitations.

Now, out of all these factors, the ones we can control and which will have the greatest effect on the ultimate quality of the final radiograph can be reduced to the exposure factors of kilovoltage, milliamperes, time, dis tance, control of scatter, and proper processing techniques. If you do not master these important controllable factors, you cannot produce a good radiograph. 


\section{Discussion}

\section{FACTORS AFFECTING RADIOGRAPHIC SENSITIVITY}

N. S. Beyer, Argonne National Laboratory: I don't want to pick out one little fact out of your detailed and very thorough talk, but I wondered if you care to comment on your statement a few moments ago that by careful examination you do seem to increase graininess with the use of lead screens.

W. D. Kiehle: Most of this work came out of our own research laboratory in which George Corney has done some of the work. We do find that lead screens inherently give a small increase in graininess, but it is not appreciable and does not affect the radiographic sensitivity of the film to any great degree. Do you have any further comment on that, George?

G. M. Corney, Eastman Kodak Company: I would say that this increase in graininess is minimal. The work was done a number of years ago when the only way we had to compare graininess of X-ray films of high graininesses, as is encountered in X-ray films, was visual. This is a statistical thing. We have a number of people judging a number of things, and saying "I think this is just a little grainier than that." Recently, the instruments used for measuring graininess have been improved to a point where it is now possible to get reliable graininess figures instrumentally, and we will certainly want to repeat this work. It has not been done as yet. But one may say that the increase in graininess due to lead foil screens is certainly minimal.

R. G. Peterson, Argonne National Laboratory: If we assume that initially the crystals are distributed uniformally throughout the emulsion, why would an increase in radiation dose change the graininess? Why do they tend to clump closer together as a function of radiation?

G. M. Corney: One can look at it in two different ways that are equivalent. As your radiation energy increases, the range of the secondary electrons generated in the emulsion will increase. One absorption will then expose more grains. Hence, you will have a bigger clump of developed grains about the site of an absorption event. The other way you can look at it gives the same answer as Mr. Larson's statistical approach. As the quantum energy increases, you transfer more energy per absorption event to the film. Hence, we need fewer of these absorption events since each is bigger, and Mr. Larson's term of $\sigma(n)$ becomes bigger.

R. G. Peterson: In other words, this is not actually a physical grouping of crystallites closer together in some limited region, but a larger area of developed grains.

G. M. Corney: That is correct. 
M. M. Turkanis, Nuclear Materials Equip. Corp.: You define a radiograph as a photograph made by means of X rays or gamma ray. This morning we saw some photographs made by neutrons. Did you purposely eliminate these from your definition of a radiograph, and if so, what do you call them?

W. D. Kiehle: This is a matter of definition. This was an omission on my part.

S. Leonard, Lockheed Aircraft Corp.: I would like to return to the apparent graininess contributed by lead screens. We at Lockheed have made microradiographs of transistors and have magnified them 150 diameters. We have noticed no contribution by lead screens to graininess. This work was done on spectrographic plate film which enables us to make these types of magnifications.

K. Lucas, Allegany Ballistics Laboratory: Is the limitation of phosphors in radiography at higher energy due primarily to which one of these two things: the proper response of phosphors at higher energy, or the graininess of the phosphors at higher energy?

W. D. Kiehle: I cannot say whether the phosphors themselves are becoming more inherently grainy due to the increase in kilovoltage. We do know that as you increase the kilovoltage the result in radiographs becomes more grainy when you're using afluorescent screen, and, therefore, it does not make it practical.

K. Lucas: Is this true even if the grain size of the fluorescent screen is smaller than that of the film?

W. D. Kiehle: I don't know of anyfluorescent screenthat has a smaller graininess than the graininess factors of the film. 


\title{
GENERATION AND DETECTION OF ULTRA-HIGH-FREQUENCY SOUND WAVES IN SOLIDS \\ by
}

\author{
H. E.Bömmel \\ Bell Telephone Laboratories, Inc. \\ (on leave to the University of California) \\ Whippany, New Jersey
}

\begin{abstract}
Ultrasonic waves have proved to be a very powerful tool for the investigation of various problems in solid state physics, such as the interaction of the lattice with dislocations, and electron phonon interaction in metals. Recently, new methodshave been developed to make it possible to extend the frequency range up to microwave frequencies. At these frequencies the acoustic wavelength in solids is of the order of $10^{-4}$ to $10^{-5} \mathrm{~cm}$, which allows the investigation of such phenomena and the interaction of the hypersonic wave with the thermal phonon with paramagnetic spin or thin wave phonon wave interaction in paramagnetic materials. A number of these problems as well as the experimental technique will be discussed.
\end{abstract}

\section{Summary}

In recent years, the measurement of the absorption and dispersion of ultrasonic waves in solids as a function of various parameters such as frequency, temperature, and magnetic field has become a very powerful tool for the investigation of many properties of the solid state.

A sound wave traveling through a solid will cause periodic deformations of the lattice, which can produce local periodic variations of density, pressure, temperature, or structure. These variations, in turn, can interact with quantities like dislocations or they can upset the local equilibrium between, for example, the conduction electrons and the thermal vibrations (phonons) in a metal, between electron spins and the thermal phonons in paramagnetic solids, between the nuclear magnetic moments and the lattice, etc. The re-establishment of equilibrium takes a finite time - the relaxation time. When this relaxation time is comparable with the period of the sound wave, the interaction becomes strongest and manifests itself in ultrasonic absorption and dispersion. Measurements of the absorption as a function of frequency, temperature, etc., can therefore reveal detailed information about the mechanisms involved. 
As an example, we may mention the ultrasonic attenuation caused by the conduction electrons in a metal.(1) This effect occurs when the electronic mean free path in the metal becomes comparable or larger than the acoustic wave length and is therefore a low-temperature phenomenon. The density variations in the lattice caused by the ultrasonic waves are then out of phase with the density variations of the conduction electrons. This results in an extremely large absorption in very pure metals, i.e., metals with high electrical conductivity, and, therefore, long electronic mean free paths. In superconducting metals this attenuation mechanism is suddenly quenched, and the attenuation drops to a very low value as soon as the metal is cooled below the transition temperature. Figure 1 shows the absorption of ultrasonic waves in a single crystal of tin as a function of temperature for 3 different frequencies. The analysis of this and related effects has given important new information about the interaction of "normal conducting" and "superconducting" electrons with the lattice ions.

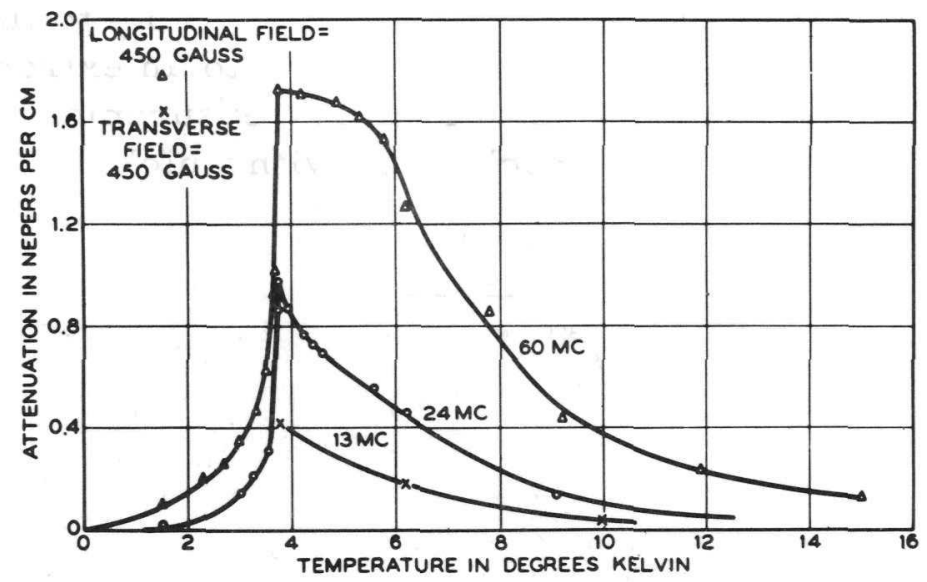

Fig. 1. Absorption of ultrasonic waves in a single crystal of tin as a function of temperature at different frequencies.

The pursuit of these kinds of investigations has recently led to an extension of ultrasonics into the microwave (hypersonic) frequency range. This has been made possible by the development of new techniques $(2)$ : piezoelectric surface excitation, magnetic uniform precession excitation, etc. The highest frequencies so far obtained are about 25 kilomegacycles/ sec. It is interesting to note that in this case the acoustic wavelength in solids becomes comparable or even smaller than the wavelength of light in the visible range (see Fig. 2).

These frequencies overlap already with the low -frequency tail of the thermal phonons, and it is therefore not surprising that a number of new and interesting effects can be observed. 


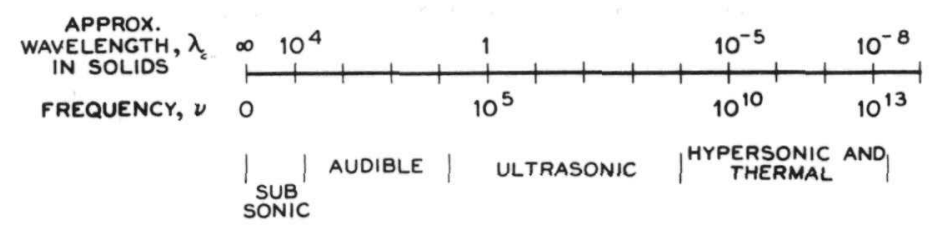

Fig. 2. Acoustic frequency spectrum. Lower scale gives frequency in cycles/sec, upper scale the corresponding average wavelength in $\mathrm{cm}$ in a solid.

A direct interaction between hypersonic and thermal waves manifests itself in a very large frequency-dependent attenuation of hypersonic waves at temperatures above about $70^{\circ} \mathrm{K}$. Below about $30^{\circ} \mathrm{K}$, where the mean free path of the thermal phonons becomes comparable to the hypersonic wavelength, this attenuation drops very rapidly to an extremely low - and frequency-independent - value (see Fig. 3). Measurements of this kind have given new insight into the mechanism with which lattice vibrations interact with each other.

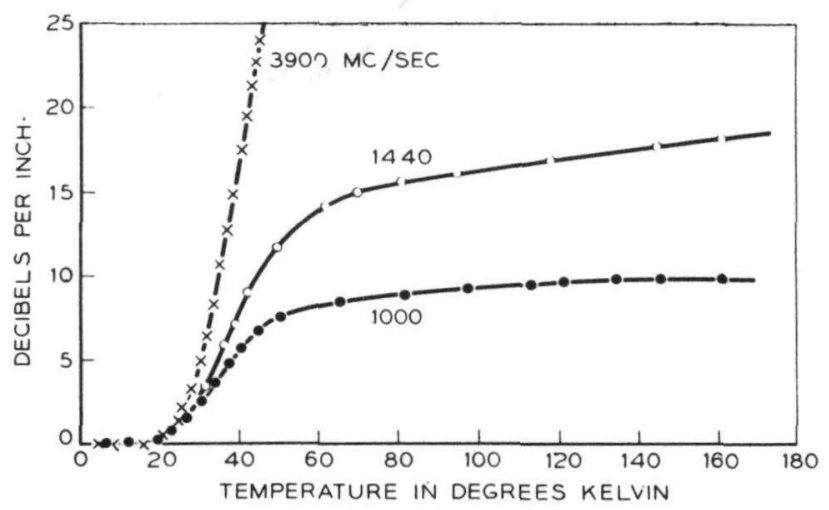

Fig. 3. Attenuation of hypersonic waves in quartz as a function of temperature at different frequencies.

Another interesting field is the interaction of hypersonic waves with paramagnetic spins. Such experiments have thrown new light on some of the still unsolved problems of paramagnetic relaxation and may in the near future make possible the development of acoustic amplifiers and acoustic masers. 


\section{References}

1. See, for example, H. E. Bömmel, Phys. Rev., 96, 220 (1954); 100 , 655 (1955), and Proc. of the Fifth International Conference on Low Temperature Physics and Chemistry, p. 272, The University of Wisconsin Press, (1958).

2. Bömmel, H. E., and K. Dransfeld, Phys. Rev. Letters, 1, 234 (1958); 2, 298 (1959), and Phys. Rev。, 117, 1245 (1960). 


\title{
ULTRASONIC PROPAGATION IN DEFORMED SINGLE AND POLYCRYSTALLINE MATERIALS*
}

\author{
by
}

\author{
F. R. Rollins \\ Midwest Research Institute \\ Kansas City, Missouri \\ and \\ R. R. Rowand \\ Aeronautical Systems Division \\ Wright-Patterson Air Force Base, Ohio
}

\begin{abstract}
Stresses have been applied to polycrystalline and single-crystal specimens to produce double refraction of megacycle shear waves. The manner in which the birefringent nature of the material varies in the "elastic" and "plastic" regions is described, and the stress-induced anisotropy which produces the effect is discussed. A pulse-echo technique for observing shear-wave double refraction is described also, and sources of error are presented.
\end{abstract}

It has been recognized for some time that the velocity and attenuation of ultrasonic waves traveling through solid materials are usually stress-dependent. The exact nature of these stress-dependent changes is not completely understood, but recent advances in dislocation theory and finite elasticity can explain many of the experimental observations. In the following paper, we will describe some experiments which illustrate the stress-dependence of ultrasonic velocities and we hope to show how the se experiments can be used in determining physical properties of the material under investigation.

The velocities of longitudinal and shear waves in an isotropic, homogeneous medium are usually given by the following expressions:

$$
\mathrm{V}_{\mathrm{L}}=\sqrt{\frac{\lambda+2 \mu}{\rho}} ; \mathrm{V}_{\mathrm{S}}=\sqrt{\frac{\mu}{\rho}},
$$

where $\lambda$ and $\mu$ are the second-order Lame' constants, and $\rho$ is the density of the medium. These expressions are derived on the basis of infinitesimal deformations. They do not predict a stress-dependent velocity so long as

* This work was supported by the Air Research and Development Command, U.S.A.F. 
the density remains constant. However, by using the finite-strain method of Murnaghan,(1) Hughes and Kelly(2) calculated the velocities of ultrasonic waves in isotropic media and obtained the stress-dependent expressions shown below:

(a) For hydrostatic pressure p:

Longitudinal

wave

$$
\rho_{0} V_{L}^{2}=(2 \mu+\lambda)-\frac{p}{3 K}[10 \mu+7 \lambda+6 l+4 m]
$$

Shear wave

$$
\rho_{0} V_{S}^{2}=\mu-\frac{p}{3 K}\left[3(2 \mu+\lambda)+3 m-\frac{n}{2}\right]
$$

(b) Uniaxial pressure $\mathrm{P}$ and wave propagation, both in the $x$ direction:

Longitudinal

wave

$$
\rho_{0} V_{L}^{2}=(2 \mu+\lambda)-\frac{P}{3 K}\left[\frac{\mu+\lambda}{\mu}(10 \mu+4 \lambda+4 m)+\lambda+2 l\right]
$$

Shear wave

$$
\rho_{0} V_{S}^{2}=\mu-\frac{P}{3 K}\left[4(\mu+\lambda)+\frac{\lambda}{4 \mu} \mathrm{n}+\mathrm{m}\right]
$$

(c) Uniaxial pressure $\mathrm{P}$ in the $\mathrm{x}$ direction with wave propagation in the y direction:

Longitudinal

wave

Shear wave with particle motion in the $\mathrm{x}$ direction

Shear wave with particle motion in the $\mathrm{z}$ direction

$$
\beta_{0} V_{L}^{2}=(2 \mu+\lambda)-\frac{P}{3 K}\left[2 l-\frac{2 \lambda}{\mu}(2 \mu+\lambda+\mathrm{m})\right]
$$

$$
\rho_{0} \mathrm{~V}_{\mathrm{S}_{1}}^{2}=\mu-\frac{\mathrm{P}}{3 \mathrm{~K}}\left[2 \mu+\lambda+\mathrm{m}+\frac{\lambda}{4 \mu} \mathrm{n}\right]
$$

$$
\rho_{0} \mathrm{~V}_{\mathrm{S} 2}^{2}=\mu-\frac{\mathrm{P}}{3 \mathrm{~K}}\left[\mathrm{~m}-2 \lambda-\frac{\mu+\lambda}{2 \mu} \mathrm{n}\right]
$$

In these expressions, $\rho_{0}$ is the density of the undeformed medium, $\mathrm{K}$ is the bulk modulus, and the terms $l, m$, and $n$ are referred to as third-order elastic constants. The latter gives an indication of the nonlinearity in the elastic behavior of a material. It is easily seen that, when the hydrostatic pressure or uniaxial stress is zero, the expressions all reduce to the two expressions given in (1). It should be pointed out that these expressions were derived for a perfectly elastic material. If plastic deformation occurs, there are other factors, such as dislocations, which may influence the ultrasonic velocity. These factors will be discussed later. 
The 3 third-order constants that appear in expressions (2)-(8) can, of course, be determined experimentally by measuring accurately the velocity changes produced in at least 3 independent cases. It is possible to independently determine the third-order constant $\mathrm{n}$ by a simple technique that does not necessitate the accurate measurement of absolute velocity. We will describe this technique first and later show some results we have obtained. When shear waves are sent through a specimen in a direction that is perpendicular to the direction of an applied uniaxial stress, expressions (7) and (8) indicate that the velocity depends upon the direction of particle motion. The initially isotropic material becomes birefringent. Pure shear waves can be propagated in this case only when the particle motion is either parallel or perpendicular to the applied load. If we subtract expression (8) from (7) and remember that $K=\frac{1}{3}(2 \mu+3 \lambda)$, we get

$$
\rho_{0} \mathrm{~V}_{\mathrm{S} 1}^{2}-\rho_{0} \mathrm{~V}_{\mathrm{S}_{2}}^{2}=-\frac{\mathrm{P}}{4 \mu}[4 \mu+\mathrm{n}]
$$

Since $\mathrm{V}_{\mathrm{S}_{1}}+\mathrm{V}_{\mathrm{S}_{2}} \cong 2 \mathrm{~V}_{0}$, we then have

$$
\mathrm{V}_{\mathrm{S}_{1}}-\mathrm{V}_{\mathrm{S} 2} \cong-\frac{\mathrm{P}}{8 \rho_{0} \mathrm{~V}_{0} \mu}[4 \mu+\mathrm{n}]
$$

or, expressed as a fractional difference,

$$
\frac{\mathrm{V}_{\mathrm{S}_{1}}-\mathrm{V}_{\mathrm{S} 2}}{\mathrm{~V}_{0}}=\frac{\Delta \mathrm{V}}{\mathrm{V}} \cong-\frac{\mathrm{P}}{8 \rho_{0} \mathrm{~V}_{0}^{2} \mu}[4 \mu+\mathrm{n}]=-\frac{\mathrm{P}}{8 \mu^{2}}[4 \mu+\mathrm{n}] .
$$

From (11) we see that the fractional difference between $\mathrm{V}_{\mathrm{S}_{1}}$ and $\mathrm{V}_{\mathrm{S}_{2}}$ is a linear function of the uniaxial stress P. Experimental determination of $\Delta \mathrm{V} / \mathrm{V}$ at a given stress is sufficient then to evaluate the third-order constant $\mathrm{n}$.

The value of $\Delta V / V$ can be determined by examining the pulse-echo patterns that occur when a plane-polarized shear wave is sent through a stressed specimen such that the propagation direction is perpendicular to the stress direction. Figure 1 illustrates the types of echo patterns that occur for various orientations of the transducer. Note that an exponential decay is obtained when the incident shear wave is polarized such that the particle motion is either parallel or perpendicular to the uniaxial stress. When the incident shear wave is polarized at an angle of approximately $45^{\circ}$ to the stress, the echo pattern exhibits an obvious minimum. This pulseecho pattern may be interpreted by considering Fig. 2, which shows the particle motion that results from the addition of two simple periodic motions of the same frequency but having displacements in orthogonal directions. Let us assume that the polarized shear wave enters a material, as shown in Fig. 2a. 


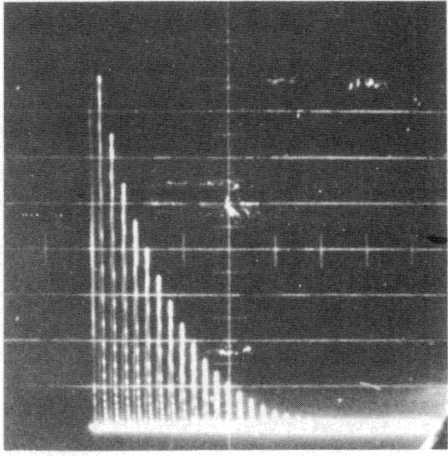

$\theta=0^{\circ}$

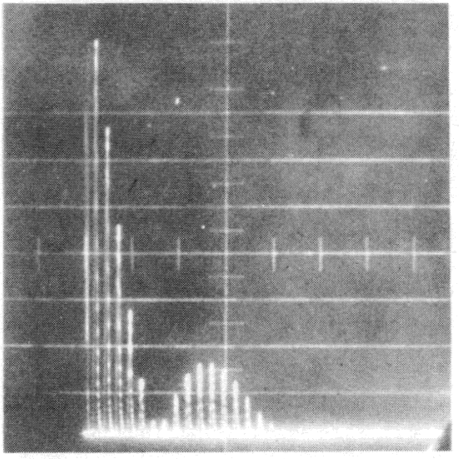

$\theta=45^{\circ}$

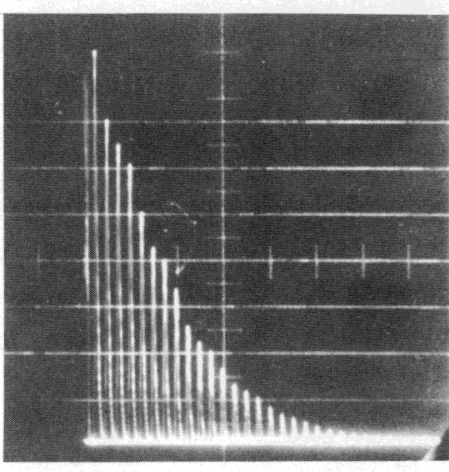

$\theta=90^{\circ}$

Fig. 1. Oscillograms of Pulse-Echo Patterns for Various Angles Between the Stress Direction and the Polarization of the Incident Shear Wave

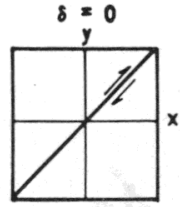

(a)

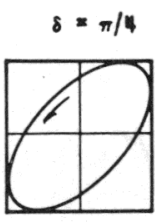

(b)

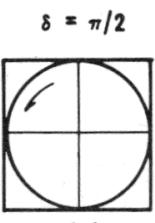

(c)

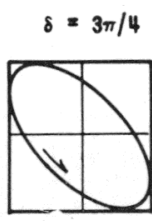

(d)

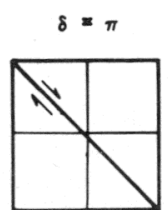

(e)

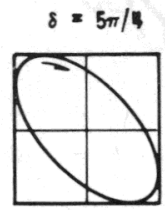

(f)

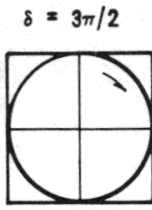

(g)

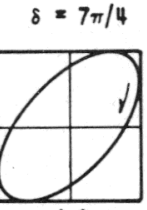

(h)

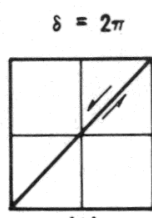

(i)

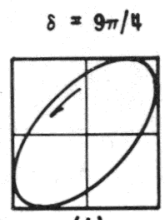

(J)

Fig. 2. Particle motion resulting from the addition of two sine waves polarized at $90^{\circ}$ to each other. The two waves have the same frequency and amplitude but phase difference, $\delta$.

If the material is anisotropic because of an applied stress, the polarized wave will be broken into components which have different velocities. The component particle motion will be along directions which we will refer to as principal axes (they do coincide with the directions of principal stress in a plane stress system). If $X$ and $Y$ are the principal axes, the diagrams in Fig. 2 show the particle motion as the wave travels through the material, and the components exhibit a phase difference $\delta$. The particle motion becomes elliptical, then circular, and finally returns to a plane-polarized condition. However, at $\delta=\pi$, the particle motion is perpendicular to the incident particle motion. It is this condition which produces a minimum in the echo pattern as shown in Fig. 1. The amplitude 
of vibration is not zero here, but the transducer which acts both as polarizer and analyzer is insensitive to shear waves with the new direction of polarization. We see that when $\delta=2 \pi$, the particle motion again becomes parallel to the transducer orientation and thus a maximum signal is generated.

Figure 3 illustrates the manner in which the pulse-echo patterns vary as the stress is changed. The position of the first minimum in the pulse-echo pattern can be used to calculate the difference in velocity between components of the shear wave along the principal axes. It is easily shown (3) that

$$
\Delta \mathrm{V} / \mathrm{V}=1 / 2 \mathrm{fT}
$$

where $f$ is the frequency and $T$ is the time of travel corresponding to the condition $\delta=\pi$. The determination of this velocity difference at a known stress makes it possible to evaluate the right-hand side of Eq. (11) and thus the third-order constant $n$.

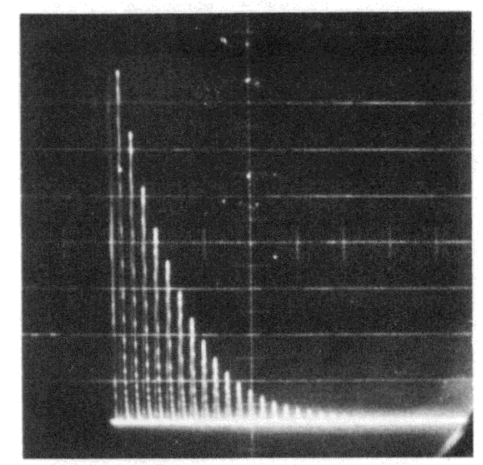

(A)

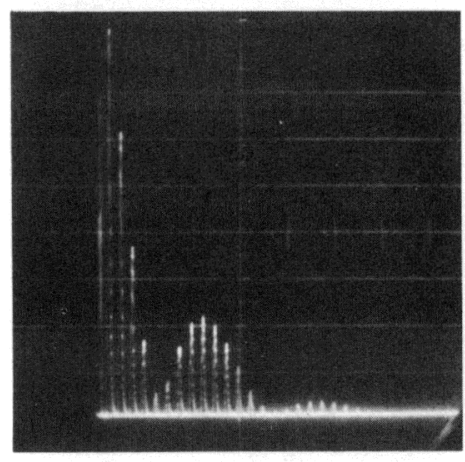

(C)

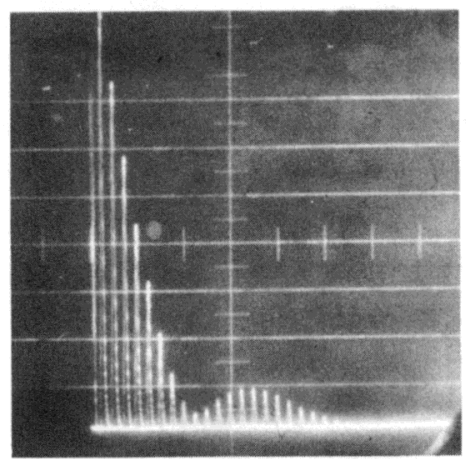

(B)

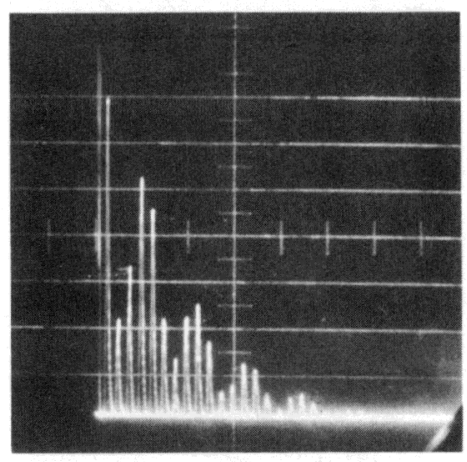

(D)

Fig. 3. Pulse-echo patterns due to increasing stress 
A typical set of data taken with a specimen of C1018 steel is shown in Fig. 4. A comparison of the stress-strain curve and the $\Delta V / V$ versus strain curve shows that the values of $\Delta V / V$ are primarily related to elas tic strain rather than to plastic strain. If we plot the fractional velocity difference versus stress, as shown in Fig. 5, the slope of the curves, as deduced from (11), is simply $-(4 \mu+\mathrm{n}) / 8 \mu^{2}$, and thus the third-order constant $\mathrm{n}$ can be evaluated.

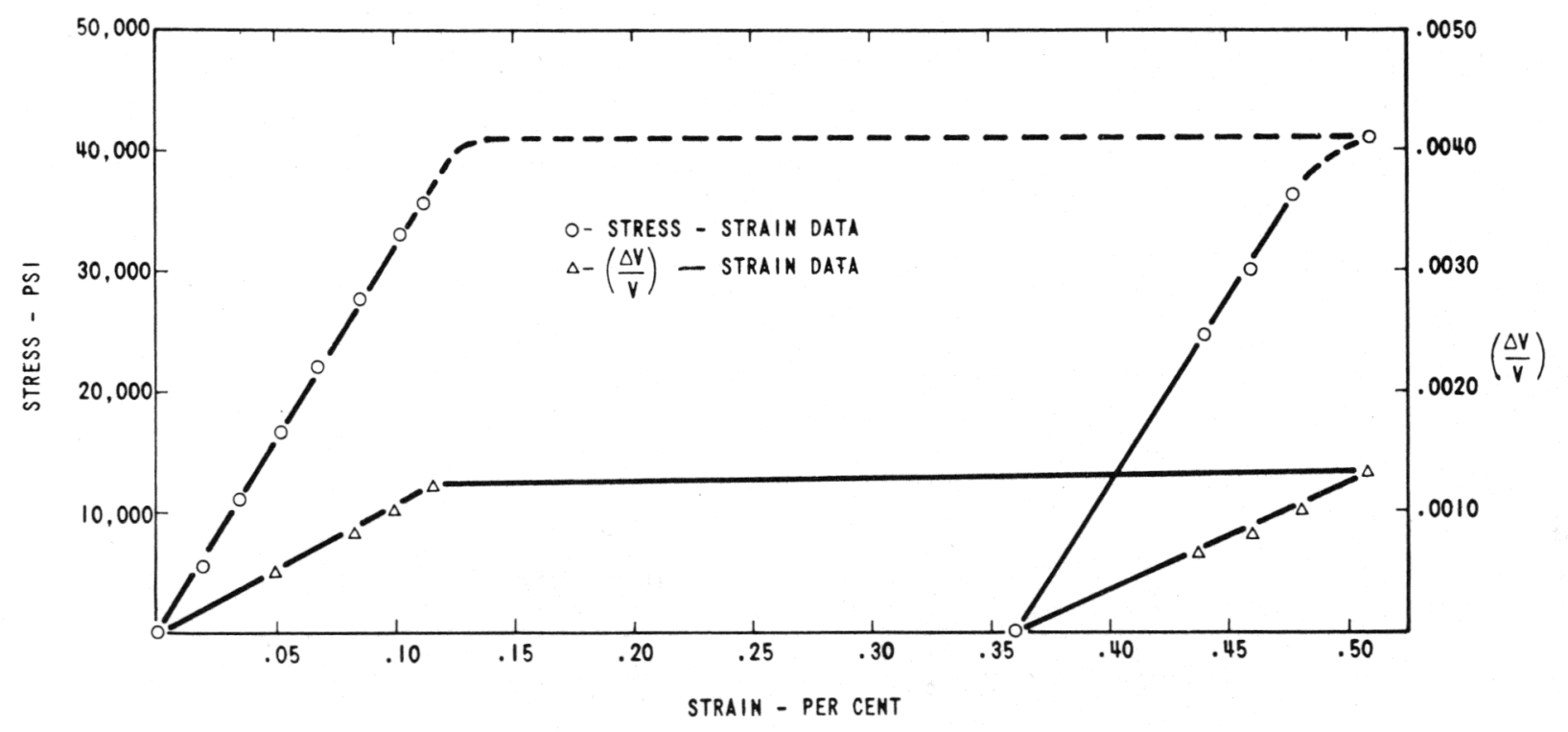

Fig. 4. Stress-strain and $\frac{\Delta V}{V}-$ Strain Curves for Cl018 Steel.

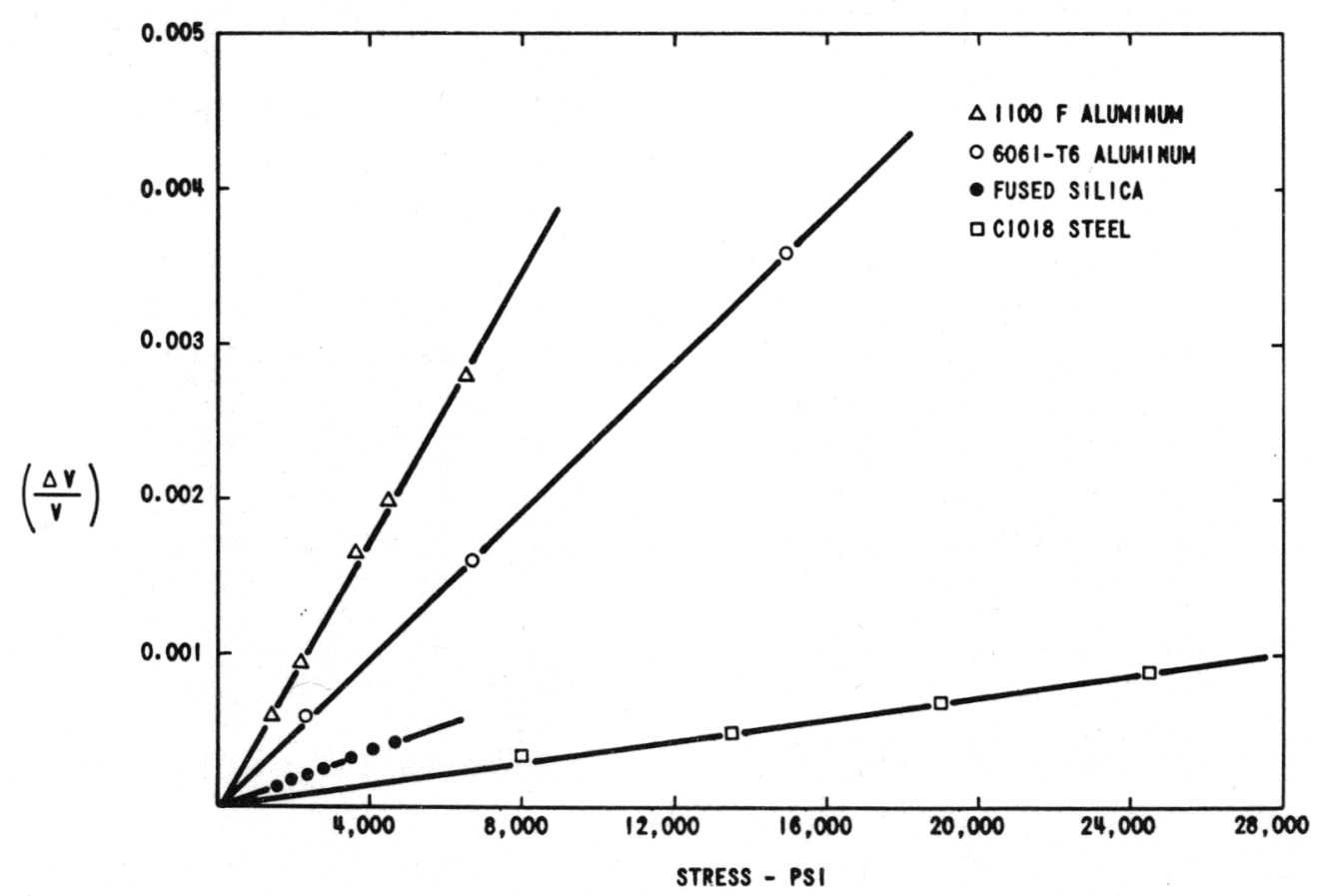

Fig. 5. Stress-induced Birefringence for Several Materials. 
For a material that is completely isotropic at zero stress, it is clear that the slope can be determined by a suitable calibration experiment and then used in subsequent experiments to relate $\Delta V / V$ to the magnitude of either an applied or a residual stress. Other anisotropic conditions, such as preferred orientation, may produce birefringence also. Such conditions often prevent a one-to-one correlation between $\Delta V / V$ and the stress level in a particular material. The two curves shown in Fig. 6, for example, represent the birefringence at two different locations on a specimen of rolled aluminum. The material was annealed prior to the test; thus, the variation in $\Delta \mathrm{V} / \mathrm{V}$ at zero stress is due primarily to preferred orientation. It should be noted that the slopes of the two curves are nearly the same even though the zero-stress conditions vary considerably.

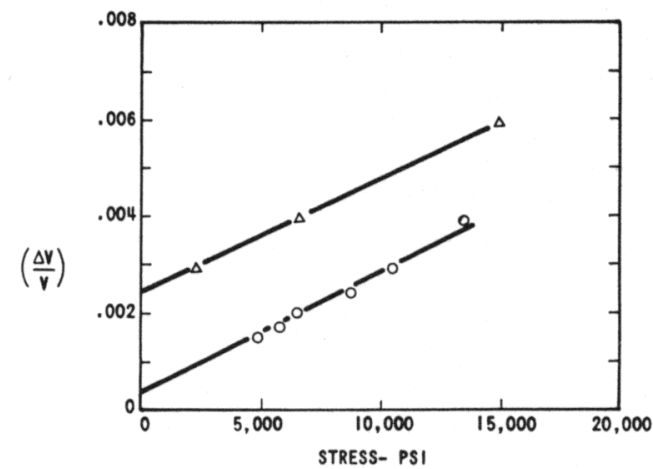

Fig. 6

$\frac{\Delta V}{V}$ versus Stress at Two Locations on an Aluminum Specimen.

The velocity of ultrasonic waves in single crystals is also stressdependent, but the velocity expressions are somewhat more involved due to the loss of symmetry. Seeger and Buck(4) have derived the differential equations for wave propagation in cubic crystals that have superimposed finite stresses. There are 6 third-order elastic constants for such crystals, and Bateman et al.,(5) have recently used ultrasonic techniques to evaluate all of these constants for germanium. Some of these constants can be evaluatedfrombirefringence experiments similar to that described for the isotropic case. The velocity expression given in Ref. 5 for a shear wave traveling in the [001] direction with particle motion parallel to a $[110]$ pressure is as follows:

$$
\begin{aligned}
\rho V_{\|}^{2}= & (1+\gamma+\alpha-\beta) C_{44}+[(\alpha+\beta) / 4] C_{144}+[(\alpha+\beta+2 \gamma) / 4] C_{166} \\
& +[(\alpha-\beta) / 4] C_{456} .
\end{aligned}
$$

For a shear wave traveling in the same direction but with particle motion perpendicular to [110], the expression is

$$
\begin{aligned}
\rho V_{\perp}= & (1+\gamma+\beta-\alpha) C_{44}+[(\alpha+\beta) / 4] C_{144}+[(\alpha+\beta+2 \gamma) / 4] C_{166} \\
& +[(\beta-\alpha) / 4] C_{456} .
\end{aligned}
$$


In the above expressions $\alpha, \beta$, and $\gamma$ are all linear functions of the applied stress, and the $\mathrm{C}_{i j k}$ terms are the third-order moduli. By using the approximation $\left(V_{\|}+V_{\perp}\right) \cong 2 \mathrm{~V}$, the birefringent nature of the crystal can be expressed as

$$
\frac{V_{\|}-V_{1}}{V} \cong-\frac{P}{8 C_{44}^{2}}\left(4 C_{44}+C_{456}\right)
$$

Here again it is possible to evaluate independently a third-order constant from only one double-refraction measurement.

We have studied stress-induced birefringence in single crystals of aluminum and sodium chloride. The results have been similar to those obtained for polycrystalline specimens, but somewhat less reproducible. There is an additional effect which one may encounter in working with single crystals: internal conical refraction. This effect occurs when shear waves travel through a crystal in a direction that is not exactly parallel to a specific crystal direction. Pure shear waves, for example, can travel through a cubic crystal in the [100] direction, and the velocity is independent of the polarization angle. If, however, the propagation direction is slightly misoriented with respect to [100], then internal conical refraction may occur. Waterman and Teutonico(3) have shown that this effect can produce pulse-echo patterns exactly like those in Figs. 1 and 3. Precise orientation of the single crystal is very important, then, in shear wave studies, particularly at high frequencies.

It was mentioned earlier that the stress-dependent velocity expressions (2)-(8) and (13)-(14) were derived for a perfectly elastic material. For most real materials, an applied stress will produce some anelastic strain in addition to the elastic strain. Dislocation motion is a good example of such anelastic strain. Granato and Lucke $(6,7)$ have developed a theory of energy losses and modulus changes that are due to dislocation damping. The theory is based on the model of a dislocation loop oscillating under the influence of an applied stress. Without going into the details of this theory, it is sufficient to state that the fractional velocity change due to the dislocations is proportional to the dislocation density and the second power of the dislocation loop length. There is quite a lot of experimental evidence that supports the Granato-Lucke theory, and there is no question that dislocations do affect the propagation velocity of ultrasonic waves. It is interesting, then, to re-examine Fig. 4 and notice that the stress-dependent birefringence did not change appreciably even when the stress exceeded the yield point. Since the dislocation density and loop lengths undoubtedly change during such deformation, the results indicate that the changes are essentially isotropic. Thus, shear waves with polarizations parallel and perpendicular to the applied stress would both undergo almost equal velocity 
changes that would be related to dislocations. The birefringence or difference between the two velocities is therefore relatively unaffected by the dislocation changes and depends primarily on the elastic strain.

The dislocation motion in single crystals occurs primarily along preferred slip planes. The dislocation-velocity relationship is then very sensitive to the crystal type, and to the directions of propagation and polarization. Nevertheless, in some cases, the dislocation effects can be minimized and the stress-dependent birefringence is primarily a function of elastic strain.

We would like to mention one additional phase of ultrasonics that is related to nonlinear elasticity. If 2 ultrasonic waves intersect each other in a linear medium, the law of superposition holds and thus there is no interaction between the waves. However, if the basic expression of elastic energy is extended to terms cubic in strains, the equations of particle motion then contain quadratic terms in the displacements. The independent nature of the intersecting waves no longer holds and the theory admits the possibility of interaction between waves.

An analysis of wave interaction in nonlinear solids has recently been completed at MRI. The analysis considers only the interaction of waves in a homogeneous, isotropic solid. The results indicate that for a given material there does exist an angle of intersection between two waves of frequencies $\omega_{1}$ and $\omega_{2}$ which produces a "resonant" interaction. At the resonant conditions, new waves are "conceived" which have frequencies of $\omega_{1} \pm \omega_{2}$. The analysis further predicts the direction of the particle motion as well as the propagation direction of the conceived wave with respect to the incident waves. We have not yet started an experimental verification of the theoretical predictions but we plan to do so soon. We feel that the detection of conceived waves which originate in subsurface volume elements may provide valuable information about the properties of the interaction volume.

\section{Bibliography}

1. F. D. Murnaghan, Finite Deformations of an Elastic Solid, John Wiley \& Sons, Inc., New York (1951).

2. D. S. Hughes, and J. L. Kelly, Phys, Rev., 92, 1145 (1953).

3. P. C. Waterman, and L. J. Teutonico, J. Appl. Phys., 28, 266 (1957).

4. A. Seeger, and O. Buck, Z. Naturforschung, 15a, 1056 (1960).

5. T. Bateman, W. P. Mason, and H. J. McSkimen, J. Appl. Phys., 32, 928 (1961).

6. A. Granato, and K. Lucke, J. Appl. Phys.,27, 583 (1956).

7. A. Granato, and K. Lucke, J. Appl. Phys., 27, 789 (1956). 


\section{Discussion \\ ULTRASONIC PROPAGATION IN DEFORMED SINGLE AND POLYCRYSTALLINE MATERIALS}

S. Serabian, Avco Corporation: You displayed a curve where you were measuring velocity as a function of deformation. On this curve there were points where the velocity difference $\Delta V / V$ was in the realm of about 0.001 or less. This brings up the question as to how you actually did measure your velocities.

F. R. Rollins: Actually, one measures the velocity difference only. This is one of the advantages of this method; one does not have to measure absolute velocity. The very low values of $\Delta \mathrm{V} / \mathrm{V}$ that you mentioned came from fused silica and mild steel. You see that you may observe the first minimum at a position that may be 15 echos out in a specimen that is 1.0 in. thick. This causes the value of $\Delta \mathrm{V} / \mathrm{V}$ to be quite small. To measure these 2 velocities absolutely and then to subtract the difference would require some extremely high-precision measurements of velocity. It would be much more difficult to do it that way than by the method we are using. This method is the standard interferometer technique used to measure, very accurately, velocity differences.

N. Sinclair, Electric Boat Division, General Dynamics Corp.: Do you have an approach for the measurement of triaxial stresses?

F.R. Rollins: No. This is an order of magnitude higher problem. Actually, we are thinking about using something like this for obtaining information about triaxial stress systems, but it certainly is not a refined situation at the present.

R. L. Chaddha, Kansas State University: You conducted 4 experiments, and I feel that you haven't really established the nature of the distribution of $n$. You had a series of figures for the third-order constant $\mathrm{n}$, and you gave $t$ figures for this. I'd just like to say that unless the dis tributional nature of this estimate is established, these \pm figures don't tell you anything.

F.R. Rollins: I did not want to indicate that the se were absolute values by any means, and they do not represent a statistical value of \pm , but merely the spread that we obtained in about 4 samples. I will stand to your point.

D. R. Green, General Electric, Hanford Atomic Products Operation: Slight changes in alignment of an ultrasonic transducer will give you differences in reflections. They give a pattern similar to the pattern shown in Fig. 1. Would you comment on special problems in alignment that you might have faced here, and how you cured them? 
F.R. Rollins: Most of these experiments were done with compressional specimens, and I would suggest that tension specimens are abetter way to avoid the problem that you point out. However, in most of our experiments, we started out with the 2 opposite surfaces parallel to within about $0.1 \mathrm{mil}$. We have no method of following the change in this value as stress is applied, but it appears, since one can obtain a recovery of the initial value of $\Delta \mathrm{V} / \mathrm{V}$ after the application of a stress great enough to cause yielding, that you have not had any serious loss in parallelism.

D.R. Green: You mentioned that you weren't in the elastic range. How do you define the region that you were in? I am not familiar enough with the particular materials you used to know just how far up the stressstrain curve you were operating.

F.R. Rollins: Most materials, I would guess, yield at a strain of about 0.1 percent. The one value which showed quite a bit of plastic yielding ran out to, I think, 0.5 percent. Engineering terminology for the elastic range, of course, is merely a description of what is normally called the proportional. There is anelasticity in the range which most people refer to as the elastic range. I think that probably answers your question, and our results actually show that this is the case. If the material were purely elastic or linear in its behavior in the range which is defined as elastic, one wouldn't expect this to happen.

G. Hake, Atomic Energy of Canada: Your corrections to your elastic constants were made as if they were independent of frequency. Shouldn't the frequency be in the expression somewhere, or were the measurements made at a discrete frequency.

F.R. Rollins: We have performed certain experiments in which the value of $\Delta \mathrm{V} / \mathrm{V}$ was correlated with stress at a range of frequencies from 5 to 15 megacycles, and you get all of the points lying on the same curve for even the different frequencies. And, of course, we do normally have a finite period after the stress is applied before the value, which we use to calculate $\Delta V / V$, is determined.

S. Serabian: Your pulse-echo patterns show a perfectly exponential curve, and then when you applied some amount of strain a minimum point appeared. The curve became exponential again, and then you noticed as you applied more strain you picked up more minimum points. At some points these minimums were occurring at the fifth to seventh reflections, and yet the velocity differences were much less than one percent. It seems to me that your velocity differences would have to be much greater than what you've indicated in order to find these differences in such early echos. I was wondering if, perhaps, you might be having bonding problems in your crystal. 
F. R. Rollins: If the minimum occurs at the first echo, for example, it will be in the neighborhood of 0.01 . I have some results that will no doubt be in the neighborhood of 0.01 . I don't think we are having bonding problems. We've done quite a bit of work using one and two crystals to check the possibility of this occurring, and I do not think it does. 
MECHANICAL VIBRATIONS IN SOLID RODS

by

\author{
Ronald G. Peterson \\ Metallurgy Division \\ Argonne National Laboratory \\ Argonne, Illinois
}

\begin{abstract}
The propagation of periodic mechanical vibrations, i.e., sound waves, in solid cylinders differs significantly from their propagation in extended solid bodies. If the radius of a solid cylinder, or rod, approaches the wavelength of the sound in the rod, the rod must be considered as a waveguide, and the relationship between the elastic moduli of the solid and the velocities of sound propagation and attenuation will differ from these relationships in extended solid bodies. Since the rod is a common form of specimen used in studies of elastic moduli of solids, many investigations have been made concerning the characteristics of sound in rods. These characteristics will be reviewed for both continuous wave propagation and for pulse propagation, especially in the megacycle range of frequencies. Experimental applications of these studies will be discussed.
\end{abstract}

Many different testing methods are used in the study of the physical properties of solid materials. X rays are used to study the insides of fabricated items; metallographic techniques enable one to observe the homogeneity and grain size of specimens; large tensile machines will test the strength and the elastic properties of materials; many other examples may easily come to mind. A well-known and well-used tool for the testing of solids is high-frequency mechanical vibrations - sound waves. The uses of high-frequency sound in solids range from flaw detection, through studies of elastic properties, $(1,2)$ up to investigations of lattice interactions with many other types of physical phenomena, like electron-phonon interactions, electron paramagnetic resonance, and radiation damage.(3)

At the moment, we are particularly interested in the use of sound waves to study the elastic properties of metals. There are definite relationships between the velocities of different types of sound waves and the elastic constants of the material in which the sound is propagated.(4) Thus, information regarding the Young's modulus, bulk modulus, Poisson's ratio, and Lamé constants, for example, may be found by examining the sound velocities. Often the sound can be propagated in a specimen while it undergoes some physical change, for example stress cycling, irradiation, or temperature variation, and the elastic properties can thus be studied as a function of this physical change. 


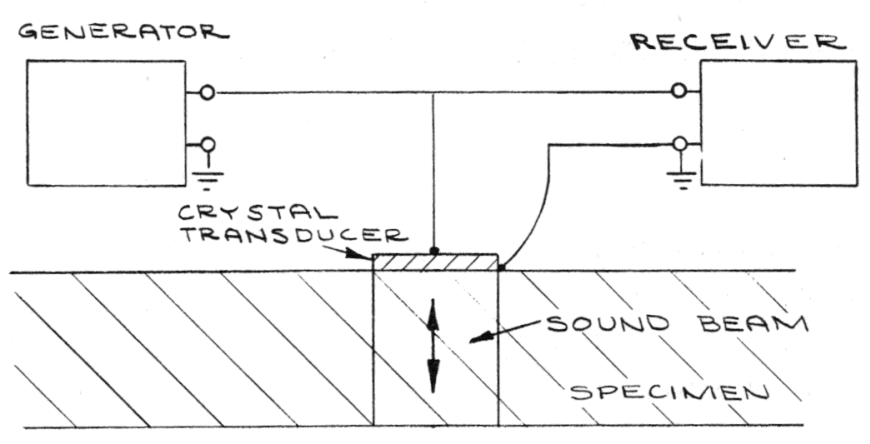

Fig. 1. Schematic Setup for Measurement of Velocities
Consider now a simplified picture of an experimental setup to measure sound velocities, as shown in Fig. 1. We show a crystal transducer radiating a sound beam into a sample, the beam being reflected from the face of the specimen opposite the transducer and returning to the crystal. The sound is produced by the generator illustrated, which may be a radiofrequency oscillator producing continuous waves or

perhaps pulses of radiofrequency oscillation; the generator may produce other types of signals such as a spike, triangular pulse, or what have you. In any case, the electrical signal from the generator is impressed across the crystal, which is piezoelectric. The vibration of the crystal in response to the electrical signal is the source of the sound wave to be propagated in the sample. For transmission of the sound from the crystal into the specimen, they are coupled together by a thin film of material which may be viscous, such as stopcock grease, or solid, for instance Eastman 910 adhesive or Salol. The sound, upon reflection from the opposite face of the specimen and returning to the crystal, causes the crystal to vibrate again, thus generating an electrical signal which is picked up by the receiver.

The received signal can be amplified and observed on some detector like a cathode-ray oscilloscope. If the signal consists of pulses, the time taken for a pulse to make one round trip through the specimen can easily be measured. After the thickness of the specimen along the beam path has been measured, the velocity can be calculated. This velocity will depend on the type of wave that is propagated into the specimen. In an isotropic solid, there are two kinds of waves to consider: longitudinal and transverse. In a longitudinal wave, also called a dilatational wave, the particle motion in the specimen is parallel to the direction of wave motion. In transverse waves, or shear waves, the particle motion is perpendicular to the wave motion. This type of wave can be polarized. In the setup illustrated in Fig. 1 the following equations apply:

$$
\begin{aligned}
c_{d} & =\sqrt{\frac{\lambda+2 \mu}{\rho}} \\
& =\sqrt{\frac{E(1-\sigma)}{\rho(1+\sigma)(1-2 \sigma)}} \\
c_{t} & =\sqrt{\frac{\mu}{\rho}} \\
& =\sqrt{\frac{E}{2 \rho(1+\sigma)}},
\end{aligned}
$$


where

$$
\begin{aligned}
\mathrm{c}_{\mathrm{d}} & =\text { longitudinal wave velocity } \\
\mathrm{c}_{\mathrm{t}} & =\text { transverse wave velocity } \\
\lambda \text { and } \mu & =\text { Lame' constants } \\
\mathrm{E} & =\text { Young's modulus } \\
\sigma & =\text { Poisson's ratio } \\
\rho & =\text { density of the specimen. }
\end{aligned}
$$

Two independent elastic constants are required to specify completely the elastic properties of an isotropic solid; all other elastic constants are combinations of whichever two you start with. The preceding velocity equations could have been written in terms of any two elastic constants. In the simple example illustrated, these are all the relationships that are needed to find the elastic properties from the wave velocity.

But in practical cases, the relationships are not so simple. In Fig. 1, we have purposely shown the specimen to be much larger than the volume included in the sound beam. We shall now add that this picture is also supposed to represent the fact that the specimen is much wider and longer than $\Lambda$, the wavelength of the sound in it. In fact, the preceding equations hold rigorously only when waves are propagated in an infinite medium. The presence of boundaries to the medium influences the way in which sound propagates. It is this influence that we shall study now. Since the solid cylinder is often used for measurements of elasticity $(2,5-7)$ and since experiments in our laboratory will be using rods also, our discussion will be primarily related to them.

For the mathematical discussion that follows we are indebted to Martin Redwood's excellent book, Mechanical Waveguides, (8) which has recently been published, and which covers the whole field of acoustic boundary value problems.

Let the point $\mathrm{P}(\mathrm{x}, \mathrm{y}, \mathrm{z})$ in an isotropic solid body be displaced by an infinitesimal amount and let $\mathrm{u}, \mathrm{v}$, and $\mathrm{w}$ represent the magnitudes of these displacements in the $x, y$, and $z$ directions, respectively. We can define two potentials which describe these displacements, a scalar potential $\rho$, and a vector potential $\psi$. These are defined by the following equations:

$$
\begin{aligned}
& u=\frac{\partial \phi}{\partial x}+\frac{\partial \psi_{z}}{\partial y}-\frac{\partial \psi_{y}}{\partial z} \\
& v=\frac{\partial \phi}{\partial y}+\frac{\partial \psi_{x}}{\partial z}-\frac{\partial \psi_{z}}{\partial x} \\
& w=\frac{\partial \phi}{\partial z}+\frac{\partial \psi_{y}}{\partial x}-\frac{\partial \psi_{x}}{\partial y}
\end{aligned}
$$


or

$$
\bar{s}=\operatorname{grad} \phi+\operatorname{curl} \psi,
$$

where

$$
\bar{s}=\hat{i u}+\hat{j v}+\hat{k w}
$$

is the infinitesimal displacement vector.

Now, if we assume Hooke's law to be true, that is, if stress is proportional to strain, then we find that these equations hold:

$$
\begin{aligned}
& \nabla^{2} \phi=\frac{1}{c_{d}^{2}} \frac{\partial^{2} \phi}{\partial t^{2}} \\
& \nabla_{i}^{2} \psi=\frac{1}{c_{t}^{2}} \frac{\partial^{2} \psi_{i}}{\partial t^{2}}
\end{aligned}
$$

where $c_{d}$ and $c_{t}$ are given by Eqs. (1) through (4).

Equations (6) are one form of the wave equation. The wave equation may also be written in terms of the displacements:

$$
\begin{aligned}
\nabla^{2} \Delta & =\frac{1}{c_{d}^{2}} \frac{\partial^{2} \Delta}{\partial t^{2}} \\
\nabla^{2} \bar{w}_{i} & =\frac{1}{c_{t}^{2}} \frac{\partial^{2} \bar{w}_{i}}{\partial t^{2}},
\end{aligned}
$$

where

$$
\begin{aligned}
\Delta=\frac{\partial u}{\partial x}+\frac{\partial v}{\partial y}+\frac{\partial w}{\partial z} & =\nabla^{2} \phi \\
& =\nabla \cdot \overline{\mathbf{s}}
\end{aligned}
$$

and

$$
\begin{aligned}
\overline{\mathrm{w}}_{\mathrm{i}} & =\left(\frac{1}{2} \nabla \mathrm{x} \overline{\mathrm{s}}\right)_{\mathrm{i}} \\
\mathrm{i} & =\mathrm{x}, \mathrm{y}, \mathrm{z} .
\end{aligned}
$$

In some cases, working with the potentials offers greater mathematical ease; in other cases working directly with the displacements is easier. 
If we try the following expressions as solution of Eqs. (6) or (6a),

$$
\begin{aligned}
& u=A_{1} \exp \left[-i \frac{2 \pi}{\Lambda}(1 x+m y+n z-c t)\right] \\
& v=A_{2} \exp \left[-i \frac{2 \pi}{\Lambda}(1 x+m y+n z-c t)\right] \\
& w=A_{3} \exp \left[-i \frac{2 \pi}{\Lambda}(1 x+m y+n z-c t)\right]
\end{aligned}
$$

we find that $c$ has a single root at $c=c_{d}$ and a double root at $c=c_{t}$. If 1 , $\mathrm{m}$, and $\mathrm{n}$ are the direction cosines of a line $\mathrm{L}$, we find that the solution $c=c_{d}$ describes a plane wave motion in the $L$ direction when the particle motion is also in this direction, while the other solutions describe plane wave motions in the $L$ direction when the particle motion is perpendicular to $L$, one solution existing for each of the two perpendicular polarization directions. Since c is the wave velocity in Eq. (7), we see that in a medium with no boundaries the longitudinal and transverse wave velocities are indeed given by Eqs. (1) through (4).

Now let our medium be a rod of radius a. At this point it becomes simpler to transform the displacements into cylindrical coordinates:

$$
\begin{aligned}
\bar{s} & =\hat{i}_{r} u_{r}+\hat{i}_{\theta} u_{\theta}+k u_{z} \\
\Delta & =\nabla \cdot \bar{s} \\
\bar{w}_{i} & =\left(\frac{1}{2} \nabla \times \bar{s}\right)_{i} \quad, \quad i=r, \theta, z
\end{aligned}
$$

In Eqs. (8), $\mathrm{z}$ is along the cylinder axis, $\mathrm{r}$ is along the cylinder radius, and $\theta$ is the azimuth measured around the axis. Let us consider a sinusoidal wave moving along the axis in the positive z direction. We can write the following equations:

$$
\begin{aligned}
& \mathrm{u}_{\mathrm{r}}=\mathrm{U}(\mathrm{r}) \cos \mathrm{n} \theta \exp \left[\mathrm{i}\left(\omega \mathrm{t}-\mathrm{k}_{0} \mathrm{z}\right)\right] \\
& \mathrm{u}_{\theta}=\mathrm{V}(\mathrm{r}) \sin \mathrm{n} \theta \exp \left[\mathrm{i}\left(\omega \mathrm{t}-\mathrm{k}_{0} \mathrm{z}\right)\right] \\
& \mathrm{u}_{\mathrm{z}}=\mathrm{W}(\mathrm{r}) \cos \mathrm{n} \theta \exp \left[\mathrm{i}\left(\omega \mathrm{t}-\mathrm{k}_{0} \mathrm{z}\right)\right] \\
& \mathrm{k}_{0}=\frac{2 \pi}{\Lambda}=\frac{\omega}{\mathrm{c}_{\mathrm{p}}}
\end{aligned}
$$

where

$\Lambda$ is the wavelength, $c_{p}$ is the phase velocity, and $\omega$ is the angular frequency. Then, $u_{r}, u_{\theta}$, and $u_{z}$ are the particle displacements in the $r$, $\theta$, and $z$ directions, as seen in Fig. 2. 


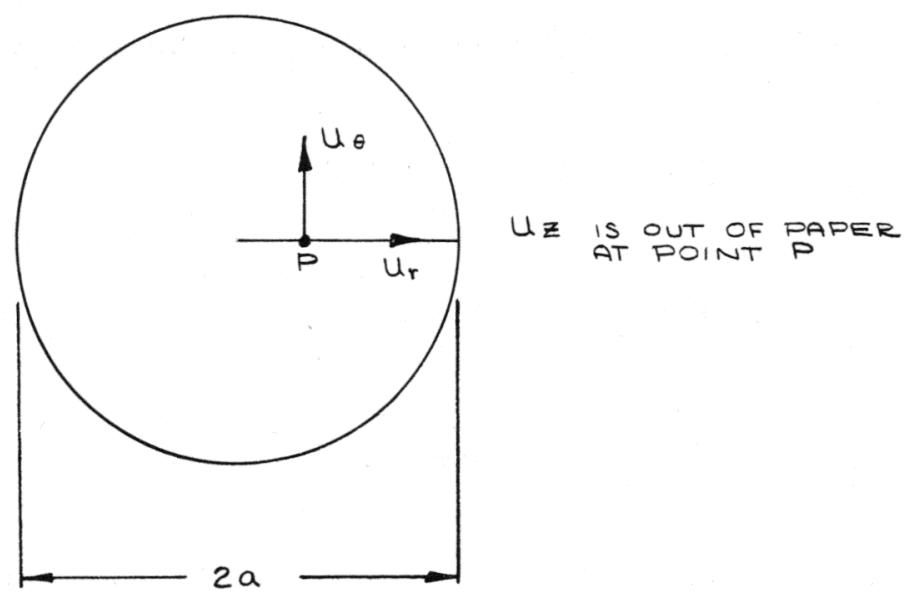

Fig. 2. Components of Particle Displacement in Cylindrical Coordinates

Putting Eqs. (6a), (8), and (9) together, we get the following solutions for the radial dependence:

$$
\begin{aligned}
& U=C\left[-A \frac{\partial}{\partial r} J_{n}\left(k_{d} r\right)+B \frac{\partial}{\partial r} J_{n}\left(k_{t} r\right)+n \frac{J_{n}\left(k_{t} r\right)}{r}\right] \\
& V=C\left[A \frac{n}{r} J_{n}\left(k_{d} r\right)-\frac{B n}{r} J_{n}\left(k_{t} r\right)-\frac{\partial}{\partial r} J_{n}\left(k_{t} r\right)\right] \\
& W=C\left[i A k_{0} J_{n}\left(k_{d} r\right)+i B \frac{k_{t}^{2}}{k_{0}^{2}} J_{n}\left(k_{t} r\right)\right]
\end{aligned}
$$

where

$$
\mathrm{k}_{\mathrm{d}}^{2}=\left(\omega / \mathrm{c}_{\mathrm{d}}\right)^{2}-\mathrm{k}_{0}^{2}
$$

and

$$
k_{t}^{2}=\left(\omega / c_{t}\right)^{2}-k_{0}^{2}
$$

Here $\mathrm{J}_{\mathrm{n}}$ is Bessel's function of order $\mathrm{n}$, and $\mathrm{A}, \mathrm{B}$, and $\mathrm{C}$ are constants.

The proper boundary conditions for this problem must now be considered. If we are talking about a solid cylinder with no external forces on it, then the proper boundary conditions are that the radial and tangential stresses at $\mathbf{r}=$ a must all be zero. The stress-strain relations are given by : 


$$
\begin{aligned}
& \tau_{\mathrm{rr}}=\lambda\left[\frac{\partial \mathrm{u}_{\mathrm{r}}}{\partial \mathrm{r}}+\frac{\mathrm{u}_{\mathrm{r}}}{\mathrm{r}}+\frac{1}{\mathrm{r}} \frac{\partial \mathrm{u}_{\theta}}{\partial \theta}+\frac{\partial \mathrm{u}_{\mathrm{z}}}{\partial \mathrm{z}}\right]+2 \mu \frac{\partial \mathrm{u}_{\mathrm{r}}}{\partial \mathrm{r}} \\
& \tau_{\theta \theta}=\lambda\left[\frac{\partial \mathrm{u}_{\mathrm{r}}}{\partial \mathrm{r}}+\frac{\mathrm{u}_{\mathrm{r}}}{\mathrm{r}}+\frac{1}{\mathrm{r}} \frac{\partial \mathrm{u}_{\theta}}{\partial \theta}+\frac{\partial \mathrm{u}_{\mathrm{z}}}{\partial \mathrm{z}}\right]+2 \mu\left(\frac{1}{\mathrm{r}} \frac{\partial \mathrm{u}_{\theta}}{\partial \mathrm{r}}+\frac{\mathrm{u}_{\mathrm{r}}}{\mathrm{r}}\right) \\
& \tau_{\mathrm{zz}}=\lambda\left[\frac{\partial \mathrm{u}_{\mathrm{r}}}{\partial \mathrm{r}}+\frac{\mathrm{u}_{\mathrm{r}}}{\mathrm{r}}+\frac{1}{\mathrm{r}} \frac{\partial \mathrm{u}_{\theta}}{\partial \theta}+\frac{\partial \mathrm{u}_{\mathrm{z}}}{\partial \mathrm{z}}\right]+2 \mu \frac{\partial \mathrm{u}_{\mathrm{z}}}{\partial \mathrm{z}} \\
& \tau_{\mathrm{r} \theta}=\mu\left(\frac{\partial \mathrm{u}_{\theta}}{\partial \mathrm{r}}-\frac{\mathrm{u}_{\theta}}{\mathrm{r}}+\frac{1}{\mathrm{r}} \frac{\partial \mathrm{u}_{\mathrm{r}}}{\partial \theta}\right) \\
& \tau_{\mathrm{rz}}=\mu\left(\frac{\partial \mathrm{u}_{\mathrm{r}}}{\partial \mathrm{z}}+\frac{\partial \mathrm{u}_{\mathrm{z}}}{\partial \mathrm{r}}\right) \\
& \tau_{\theta \mathrm{z}}=\mu\left(\frac{1}{\mathrm{r}} \frac{\partial \mathrm{u}_{\mathrm{z}}}{\partial \theta}+\frac{\partial \mathrm{u}_{\theta}}{\partial \mathrm{z}}\right)
\end{aligned}
$$

By $\tau_{i j}$ is meant the stress acting in the $\mathrm{j}$ th direction on the plane normal to the $i$ th direction. For the stress to vanish on the cylinder wall, Eqs. (15) hold:

$$
\begin{aligned}
& \left.\tau_{\mathrm{rr}}\right|_{\mathrm{r}=\mathrm{a}}=0 \\
& \left.\tau_{\mathrm{r} \theta}\right|_{\mathrm{r}=\mathrm{a}}=0 \\
& \left.\tau_{\mathrm{rz}}\right|_{\mathrm{r}=\mathrm{a}}=0 .
\end{aligned}
$$

Three equations result from the combination of (9), (11), and (15); the three unknowns $\mathrm{A}, \mathrm{B}$, and $\mathrm{C}$ may be eliminated to give the determinant in Eq. (16).

$$
0=\left|\begin{array}{llc}
n^{2}-1-a^{2} k_{0}^{2}(x-1) & n^{2}-1-a^{2} k_{0}^{2}(2 x-1) & 2\left(n^{2}-1\right)\left[\gamma_{n}\left(k_{t} a\right)-n\right]-a^{2} k_{0}^{2}(2 x-1) \\
\gamma_{n}\left(k_{d} a\right)-n-1 & \gamma_{n}\left(k_{t} a\right)-n-1 & 2 n^{2}-2\left[\gamma_{n}\left(k_{t} a\right)-n\right]-a^{2} k_{0}^{2}(2 x-1) \\
\gamma_{n}\left(k_{d} a\right)-n & -(x-1)\left[\gamma_{n}\left(k_{t} a\right)-n\right] & n^{2}
\end{array}\right|
$$

Here,

$$
\begin{aligned}
& \gamma_{n}\left(k_{a}\right)=\frac{k_{a} J_{n-1}\left(k_{a}\right)}{J_{n}\left(k_{a}\right)} \\
& x=\frac{1}{2}\left(\frac{c_{p}}{c_{t}}\right)^{2} \\
& n=0,1,2, \ldots
\end{aligned}
$$


Equation (16) is called the frequency equation for vibration of a circular cylinder. It is supposedly the most general equation relating the frequency of vibration to its wavelength. This equation was first derived and studied by L. Pochhammer(9) in 1876 and again independently by C. Chree(10) in 1889. This solution was not made use of until the early nineteen-forties; since then there have been many papers offering numerical solutions corresponding to specific physical situations. In general, the relation between frequency and wavelength changes, that is, the phase velocity $c_{p}$ given by Eq. (10) is not constant, but changes with frequency.

The amplitudes A and B in Eq. (11) can now be solved. After all these solutions have been determined, a plot of phase velocity versus wavelength or frequency can be made, and a plot of displacements (radial, angular and axial) can be made for any frequency at any point in the bar. From a knowledge of the behavior of the phase velocity $c_{p}$ with wavelength, one can determine the group velocity $\mathrm{c} g$ by the relation given in Eq. (20):

$$
\begin{aligned}
c_{g} & =\frac{d \omega}{d k_{0}} \\
& =c_{p}-\Lambda \frac{d c_{p}}{d \Lambda} .
\end{aligned}
$$

The group velocity is what is usually measured in any experiment involving wave velocity.

In order that this discussion be more physically meaningful, we shall discuss some specific examples of particular vibrations. Suppose in Eq. (9) we put $n=0, u_{r}=0$ and $u_{z}=0$. We then have

$$
u_{\theta}=V(r) \exp \left[i\left(\omega t-k_{0} z\right)\right] .
$$

This represents a motion of the particles in the rod such that there is no motion parallel to the rod axis and no motion along any radius. The only motion is a twisting of the rod, and even this is undistorted over a given cross section, since there is no dependence on azimuth angle $\theta$. These types of waves are called torsional waves. We could find our frequency equation by substituting these new expressions into Eq. (16), of course, but for this simple case it might be more instructive to proceed from Eq. (6a). The one resulting equation of motion is

$$
\frac{\partial^{2} \mathrm{~V}}{\partial \mathrm{r}^{2}}+\frac{1}{\mathrm{r}} \frac{\partial \mathrm{V}}{\partial \mathrm{r}}-\frac{1}{\mathrm{r}^{2}} \mathrm{~V}+\left[\left(\frac{\omega}{\mathrm{c}_{\mathrm{t}}}\right)^{2}-\mathrm{k}_{0}^{2}\right] \mathrm{V}=0 \text {, }
$$

and the solution is

$$
\mathrm{V}(\mathrm{r})=\mathrm{AJ}_{1}\left(\mathrm{k}_{\mathrm{t}} \mathrm{r}\right)
$$


where

$$
\mathrm{k}_{\mathrm{t}}^{2}=\left(\omega / \mathrm{c}_{\mathrm{t}}\right)^{2}-\mathrm{k}_{0}^{2}
$$

Putting in the previous boundary condition gives us the following frequency equation:

$$
\mathrm{J}_{0}\left(\mathrm{k}_{\mathrm{t}} \mathrm{a}\right) / \mathrm{J}_{1}\left(\mathrm{k}_{\mathrm{t}} \mathrm{a}\right)=2 / \mathrm{k}_{\mathrm{t}} \mathrm{a}
$$

There are an infinite number of roots to this equation. Each root corresponds to what is known as a mode of torsional vibration. For each mode, one can plot a curve of frequency versus velocity (or wavelength versus velocity). The rod may be vibrating in one or in several modes at once, i.e., the equation of motion may be more general than Eq. (21):

$$
u_{\theta}=\sum_{m=1}^{q} v_{m}(r) \exp \left[i\left(\omega t-k_{0} z\right)\right],
$$

where

$$
\mathrm{V}_{\mathrm{m}}(\mathrm{r})=\mathrm{A}_{\mathrm{m}} \mathrm{J}_{1}\left(\mathrm{k}_{\mathrm{tm}} \mathrm{r}\right)
$$

and where $\mathrm{k}_{\mathrm{tm}}$ is the $\mathrm{m}$ th root of Eq. (24).

The values of the amplitudes $A_{m}$ will depend on how the rod is excited initially. One root of Eq. (24) is $\mathrm{k}_{\mathrm{t}}=0$; calling this the first mode, we find that

$$
\mathrm{V}_{1}(\mathrm{r})=\mathrm{A}_{1} \mathrm{r},
$$

since the differential Eq. (22) becomes, if $\mathrm{k}_{\mathrm{t}}=0$,

$$
\frac{\partial^{2} V}{\partial r^{2}}+\frac{1}{r} \frac{\partial V}{\partial r}-\frac{1}{r^{2}} V=0
$$
Eqs. (29):

Thus, the complete solution for the torsional modes are given by

$$
\begin{aligned}
& u_{\theta_{1}}=A_{1} r \exp \left[i\left(\omega t-\frac{\omega}{c_{t}} z\right)\right], \quad \begin{array}{l}
m=1 \\
k_{t}=0
\end{array} \\
& u_{\theta m}=A_{m} J_{1}\left(k_{t} r\right) \exp \left[i\left(\omega t-k_{0} z\right)\right] \quad, \quad k_{t} \neq 0 . .
\end{aligned}
$$

Remembering that $\mathrm{k}_{0}=\omega / \mathrm{c}_{\mathrm{p}}$, we see that the first mode represents what is called a nondispersive wave, that is, its phase velocity is independent of frequency. We see from Eq. (29) that $c_{p}=c_{t}$. The other modes are all dispersive. 
A type of wave of more interest to us in this laboratory is one that is not quite as simple as the torsional waves just discussed. In this type of wave motion, there is no $\theta$ component of vibration, but there is an axial component and a radial component. Again we will assume axial symmetry, and the equation for particle motion will not be dependent on $\theta$.

Referring back to Eqs. (5), we see that if we choose potential such that

$$
\begin{aligned}
\phi & =\phi(\mathrm{r}, \mathrm{z}, \mathrm{t}) \\
\psi_{\mathrm{r}} & =0=\psi_{\mathrm{z}} \\
\psi_{\theta} & =\psi_{\theta}(\mathrm{r}, \mathrm{z}, \mathrm{t}),
\end{aligned}
$$

then we get

$$
\begin{aligned}
& u_{r}=\frac{\partial \phi}{\partial r}-\frac{\partial \psi \theta}{\partial z} \\
& u_{\theta}=0 \\
& u_{z}=\frac{\partial \theta}{\partial z}+\frac{1}{r} \psi_{\theta}+\frac{\partial \psi_{\theta}}{\partial r}
\end{aligned}
$$

where $u_{r}, u_{\theta}, u_{z}$ now have the properties discussed previously. We now introduce a new potential $\mathrm{R}$ in place of $\psi \theta$, such that

$$
\psi_{\theta}=-\frac{\partial R}{\partial r}
$$

Equations (6) now become

$$
\begin{aligned}
& \frac{\partial^{2} \phi}{\partial \mathrm{r}^{2}}+\frac{1}{\mathrm{r}} \frac{\partial \phi}{\partial \mathrm{r}}+\frac{\partial^{2} \phi}{\partial \mathrm{z}^{2}}=\frac{1}{\mathrm{c}_{\mathrm{d}}^{2}} \frac{\partial^{2} \phi}{\partial t^{2}} \\
& \frac{\partial^{2} \mathrm{R}}{\partial \mathrm{r}^{2}}+\frac{1}{\mathrm{r}} \frac{\partial \mathrm{R}}{\partial \mathrm{r}}+\frac{\partial^{2} \mathrm{R}}{\partial \mathrm{z}^{2}}=\frac{1}{\mathrm{c}_{\mathrm{t}}^{2}} \frac{\partial^{2} \mathrm{R}}{\partial \mathrm{t}^{2}}
\end{aligned}
$$

Putting

$$
\begin{aligned}
& \phi=\phi_{0}(r) \exp \left[i\left(\omega t-k_{0} z\right)\right] \\
& R=R_{0}(r) \exp \left[i\left(\omega t-k_{0} z\right)\right],
\end{aligned}
$$


we get

$$
\begin{aligned}
& \frac{\partial^{2} \phi_{0}}{\partial \mathrm{r}^{2}}+\frac{1}{\mathrm{r}} \frac{\partial \phi_{0}}{\partial \mathrm{r}}+\left[\left(\frac{\omega}{c_{\mathrm{d}}}\right)^{2}-\mathrm{k}_{0}^{2}\right] \phi_{0}=0 \\
& \frac{\partial^{2} \mathrm{R}_{0}}{\partial \mathrm{r}^{2}}+\frac{1}{\mathrm{r}} \frac{\partial \mathrm{R}_{0}}{\partial \mathrm{r}}+\left[\left(\frac{\omega}{c_{t}}\right)^{2}-\mathrm{k}_{0}^{2}\right] \mathrm{R}_{0}=0
\end{aligned}
$$

Before discussing the solution to the equations, let us consider the physics of the situation in order to have some feel for what is happening in the bar. Suppose this bar has a piezoelectric crystal transducer at one end, and suppose that the crystal is vibrating continuously. The bar is thus "filled" with sound waves. [We have not considered the stress on the end faces of the bar; it has been shown that, if the bar is more than a few diameters long, neglecting the end faces introduces no appreciable error. (12)] If the crystal is vibrating in the thickness mode, that is, if the two opposite faces of the crystal are alternately approaching and receding from each other in response to the electrical signal impressed across the crystal, we might at first expect that a plane longitudinal wave would be propagated down the length of the rod, similar to the case illustrated by Fig. 1. But in the bar configuration, with the stress-free cylindrical surface, we have to consider the lateral contraction produced by an axial extension in the bar. The relationship between these 2 quantities is given by Poisson's ratio. That is why we cannot now speak of a pure longitudinal wave: as soon as a longitudinal strain does occur in the bar, there is associated with it a corresponding lateral strain. From these considerations it appears plausible that we might be able mathematically to separate the resulting vibration into a part representing a longitudinal wave and a part representing a transverse wave. It turns out that this is true; a consideration of the solution to Eq. (35) reveals that the total vibration may be considered as a partly longitudinal wave incident at an angle ad to the cylindrical wall, and as a partly transverse wave incident at an angle $a_{t}$, where

$$
\begin{aligned}
& \sin a_{d}=c_{d} / c_{p}\left(k_{d} \text { real }\right) \\
& \sin a_{t}=c_{t} / c_{p}\left(k_{t} \text { real }\right) .
\end{aligned}
$$

At a particular point on the radial boundary of the bar, we may have a situation like that illustrated in Fig. 3. For this situation, that is, if at every point in the bar at every moment we have 4 waves to consider, one incident longitudinal wave, one incident transverse wave, a reflected longitudinal wave and a reflected transverse wave, then the following equations are solutions of Eq. (35):

$$
\begin{aligned}
& \phi=A J_{0}\left(k_{d} r\right) \exp \left[i\left(\omega t-k_{0} z\right)\right] \\
& R=B J_{0}\left(k_{t} r\right) \exp \left[i\left(\omega t-k_{0} z\right)\right]
\end{aligned}
$$




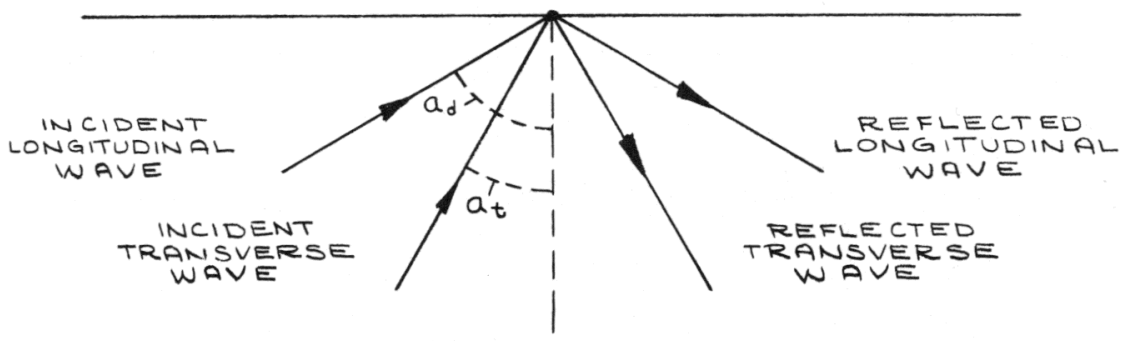

Fig. 3. Reflections at Radial Boundary of Cylinder

After applying the radial boundary conditions as before, we derive a frequency equation for this type of wave:

$$
0=k_{0}^{2} \frac{k_{t} J_{0}\left(k_{t} a\right)}{J_{1}\left(k_{t} a\right)}-\frac{1}{2}\left(\frac{\omega}{c_{t}}\right)^{2} \frac{1}{a}+\left[\frac{1}{2}\left(\frac{\omega}{c_{t}}\right)^{2}-k_{0}^{2}\right]^{2} \frac{J_{0}\left(k_{d} a\right)}{k_{d} J_{1}\left(k_{d} a\right)} .
$$

Solving this numerically allows us to plot the velocity as a function of frequency or wavelength. Bancroft(13) did this in 1941, and we see his results in Fig. 4. Here we see phase velocity for the first mode versus the ratio of diameter to wavelength. The vertical scale is plotted in terms of the ratio of phase velocity to the so-called "Young's modulus velocity,"

$$
\mathrm{v}_{0}=\mathrm{c}_{0}=\sqrt{\mathrm{E} / \rho} \text {. }
$$

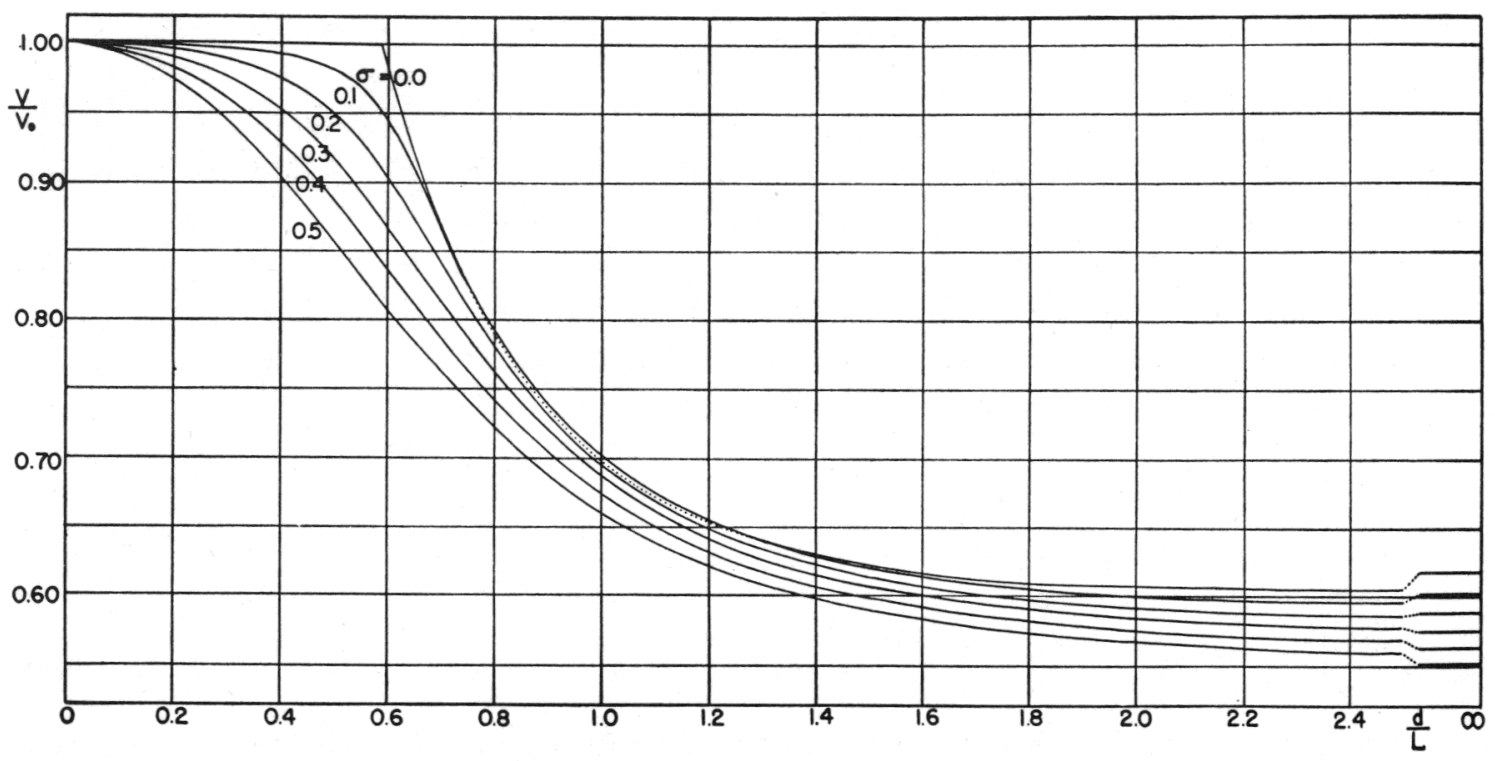

Fig. 4. Ratio of Phase Velocity, v, to Young's Modulus Velocity, vo, as a Function of the Ratio of Diameter, d, to Wavelength, L, for Various Values of Poisson's Ratio, $\sigma$.

This is the velocity at zero frequency. As can be seen, frequency increases to the right. The plot is made for several values of Poisson's ratio $\sigma$. At the extreme right-hand side of the graph are shown the 
asymptotic values at zero wavelength. Davies(6) in 1948 plotted two more modes, as shown in Fig. 5. His graph is similar to Bancroft's, but Davies only used one value of Poisson's ratio, $\sigma=0.29$. This is the value for most common steels. Curve la on the graph is given by an approximate theory, valid only at low frequencies. Note that the phase velocity becomes infinite for the second and third modes below certain frequencies. These modes have a definite cutoff frequency, below which they do not propagate.

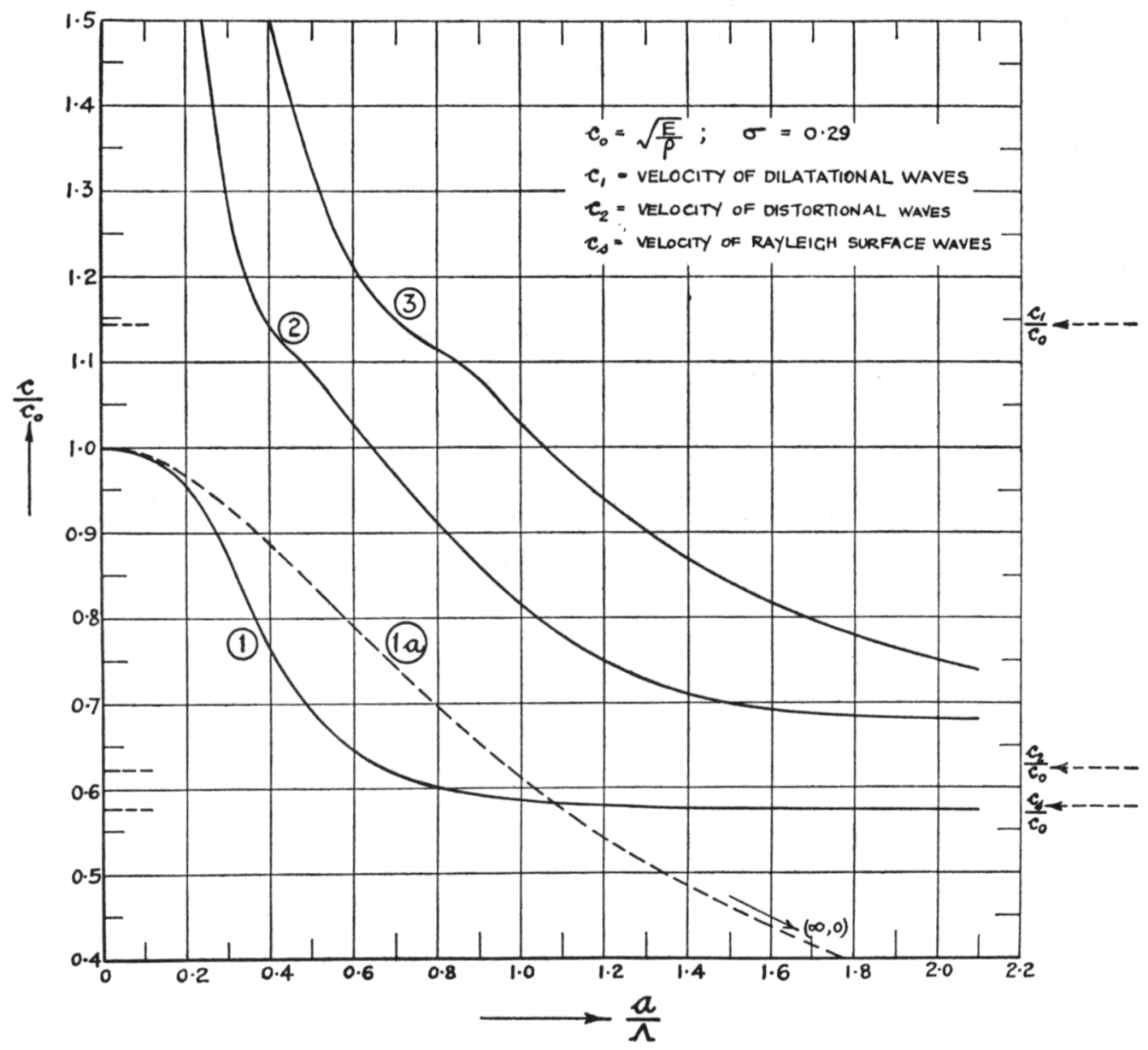

Fig. 5. Ratio of Phase Velocity, c, to Young's Modulus Velocity $\mathrm{c}_{0}$, as a Function of Radius, a, to $\Lambda$, Wavelength. First Three Modes.

Figure 6 is Davies' plot of group velocity for the first two modes. In Fig. 7 we see Bancroft's plot of the axial component of displacement along the bar radius for various values of $d / \Lambda$. This is shown for $\sigma=1 / 4$. Sometimes the displacement is greater at the surface than at the axis, sometimes the reverse is true. Notice also the vibrational nodes, for which the displacements reverse their directions. In Fig. 8, Davies has given us two pictures, at two values of a/ $\Lambda$, of the stresses as well as the displacements. 


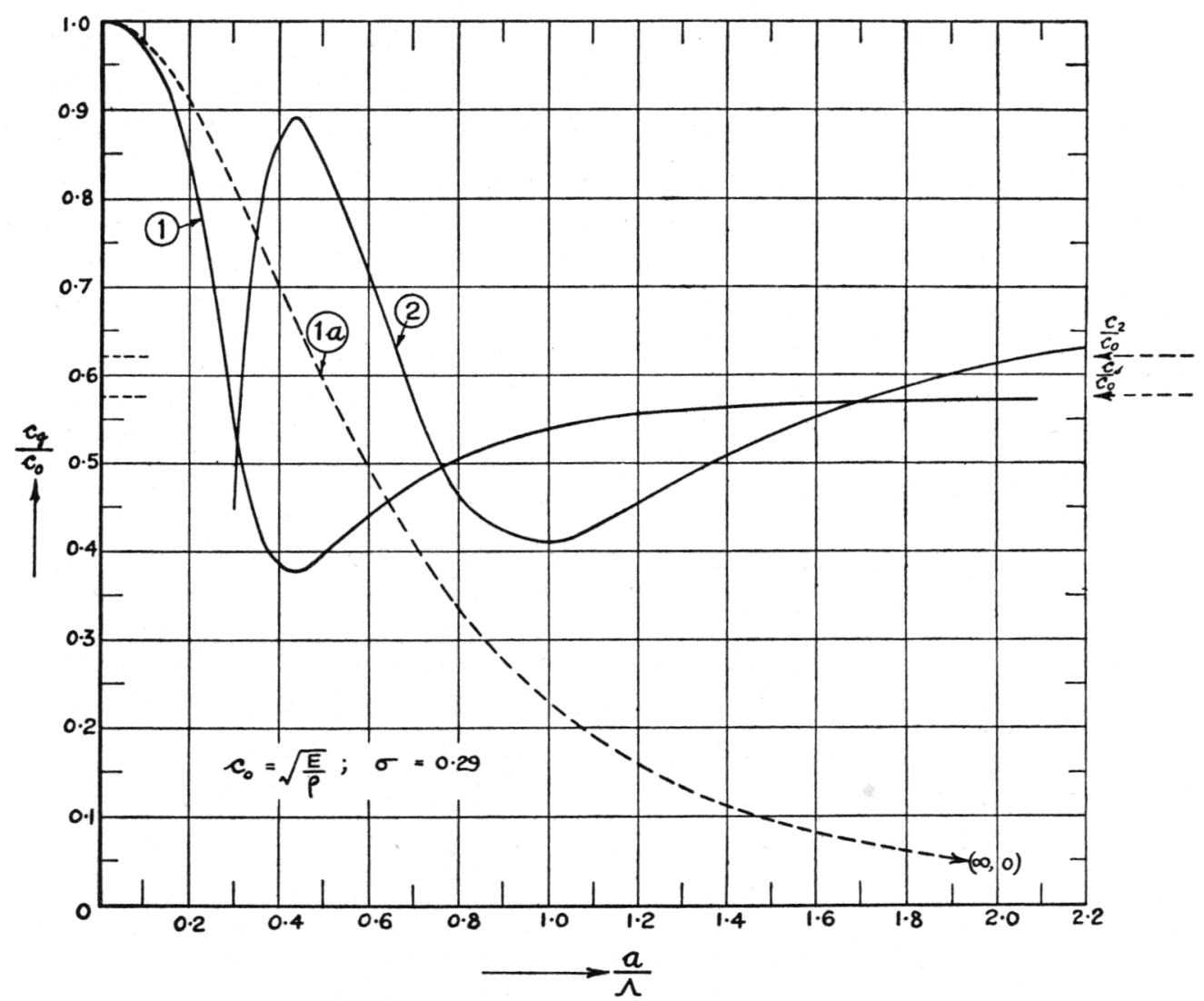

Fig. 6. Ratio of Group Velocity, cg, to Young's Modulus Velocity $\mathrm{c}_{0}$, as a Function of Radius, a, to $\Lambda$, Wavelength. First Two Modes.

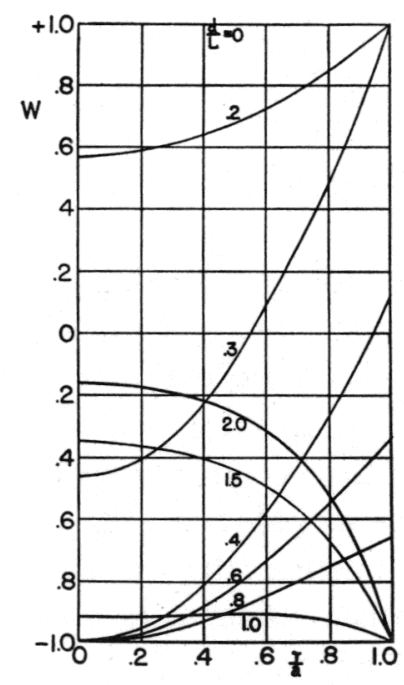

Fig. 7

Axial component of the displacement, w, as a function of ratio of radial distance, $r$, to total bar radius, a. For various values of ratio of diameter, $d$, to wavelength, $L$. 


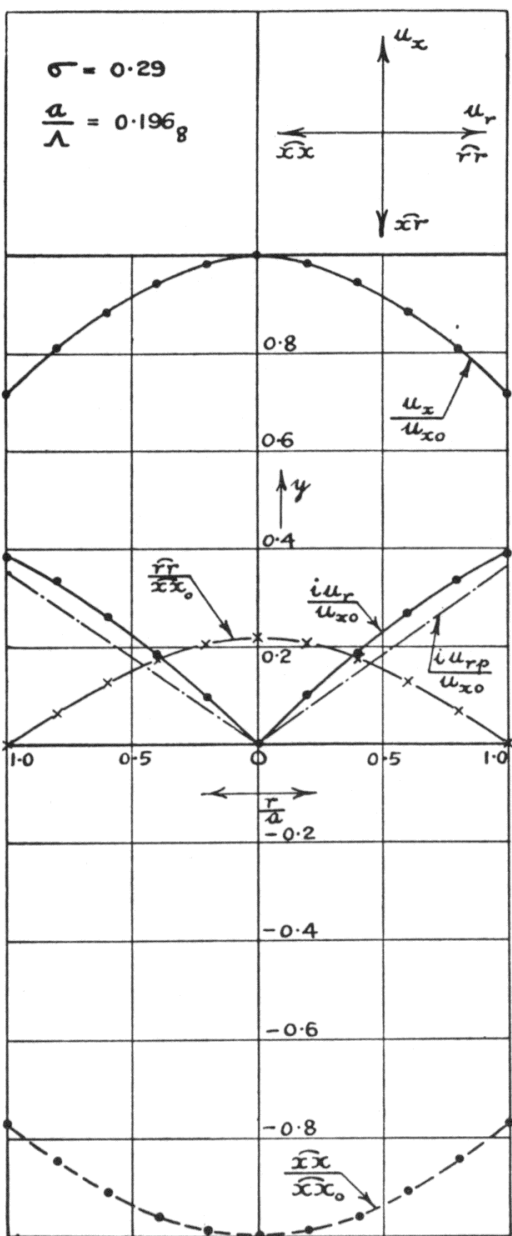

(a)

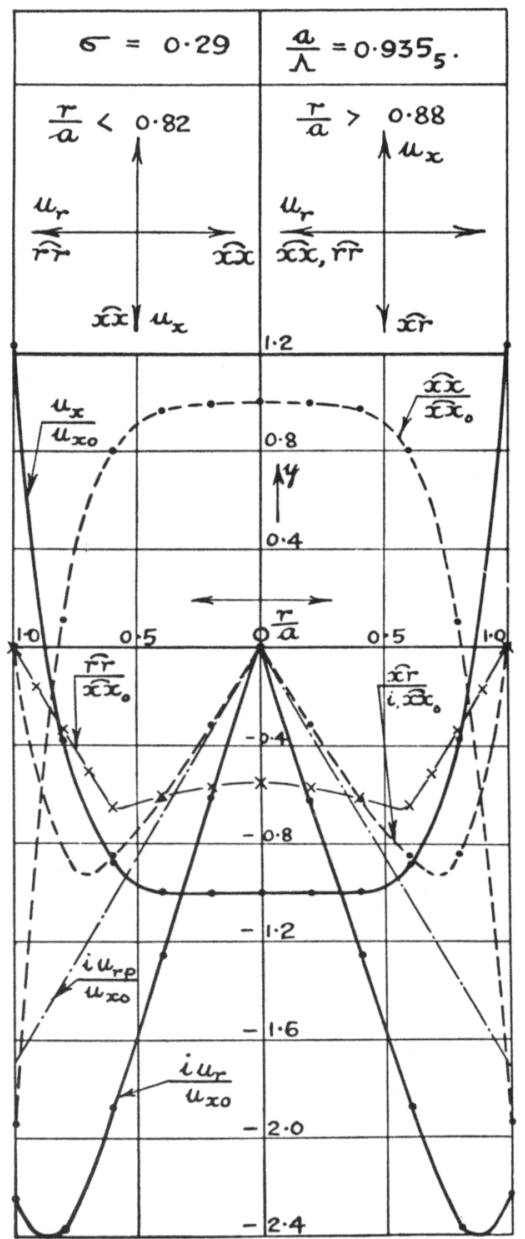

(b)

Fig. 8. The variation of the displacements and the stresses over the cross-section of a bar as a function of the ratio of radial distance, $r$, to total bar radius, a. For two values of ratio of a to wavelength $\Lambda$.

Here $\overparen{\mathrm{xx}}=$ longitudinal stress, $\overparen{\mathrm{xr}}=$ shearing stress, $\overparen{\mathrm{rr}}=$ radial stress, $\mathrm{u}_{\mathrm{x}}=$ longitudinal displacement, $\mathrm{u}_{\mathrm{r}}=\mathrm{radial}$ displacement, and $\mathrm{u}_{\mathrm{rp}}=\mathrm{radial}$ displacement in a distortionless bar. McSkimin(14) recently gave us some more plots of displacements, which may be seen in Figs. 9 and 10 . In Fig. 11, McSkimin plots the displacements for an imaginary argument $\mathrm{k}_{\mathrm{d}}=\mathrm{i} 28.5$. His comment is that the se displacements are such that the usual sort of crystal transducer would never excite this particular mode. In Fig. 12, by Redwood, (15) are shown some modes near the value $\mathrm{c}_{\mathrm{p}} / \mathrm{c}_{\mathrm{d}}=1$. These show a "plateau with steps" behavior at high frequencies. 

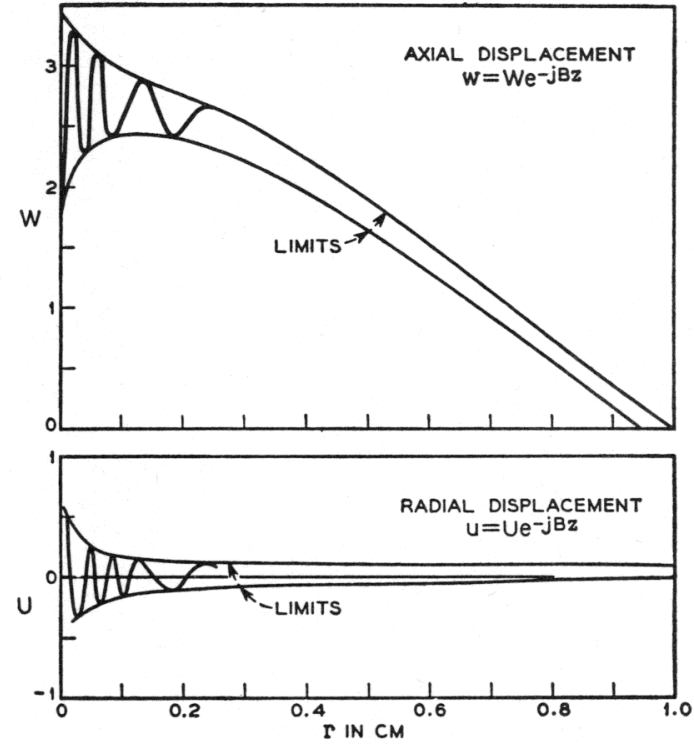

Fig. 9

First mode particle displacements for high frequency longitudinal waves in a magnesium rod. Frequency $=10$ megacycles $/$ sec; radius $=1 \mathrm{~cm}$.
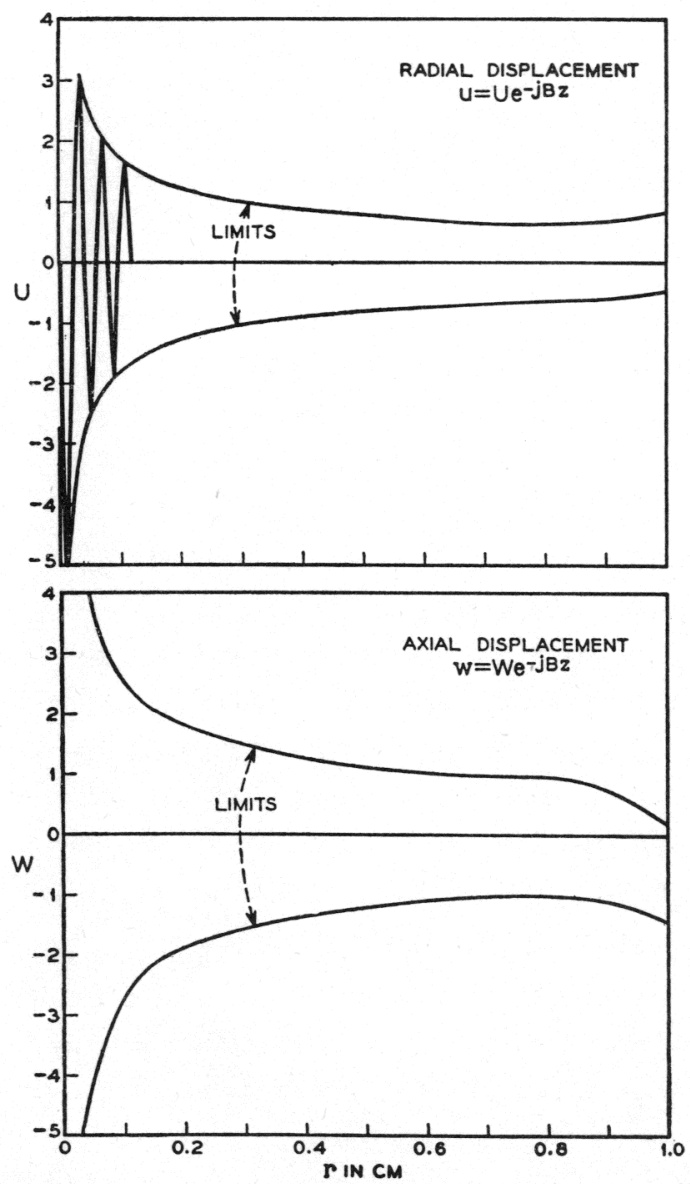

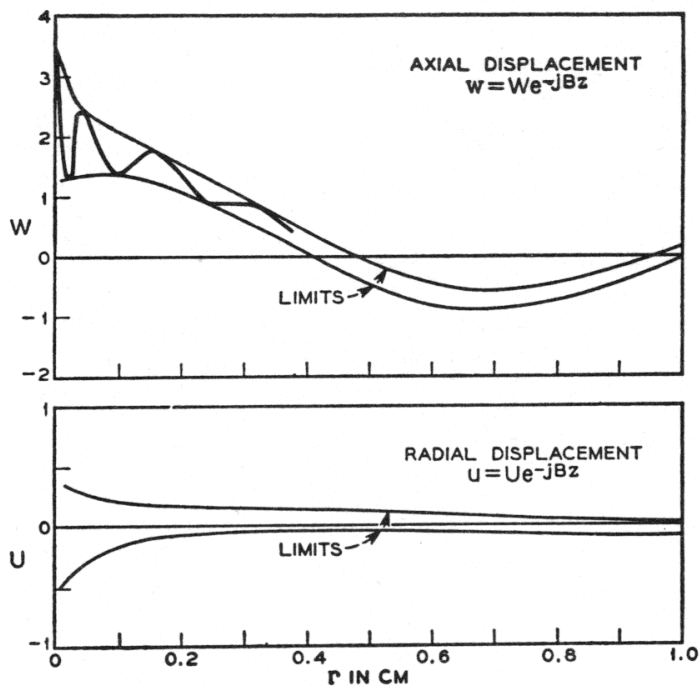

Fig. 10

Second mode particle displacements for high frequency longitudinal waves in magnesium. Frequency $=10$ megacycles / sec; radius $=1 \mathrm{~cm}$.

Fig. 11

Particle displacement for a longitudinal mode with imaginary argument $\left(\mathrm{k}_{\mathrm{d}}=\right.$ i 28.5); in a magnesium rod. Frequency = $10 \mathrm{megacycles} / \mathrm{sec}$; radius $=1 \mathrm{~cm}$. 


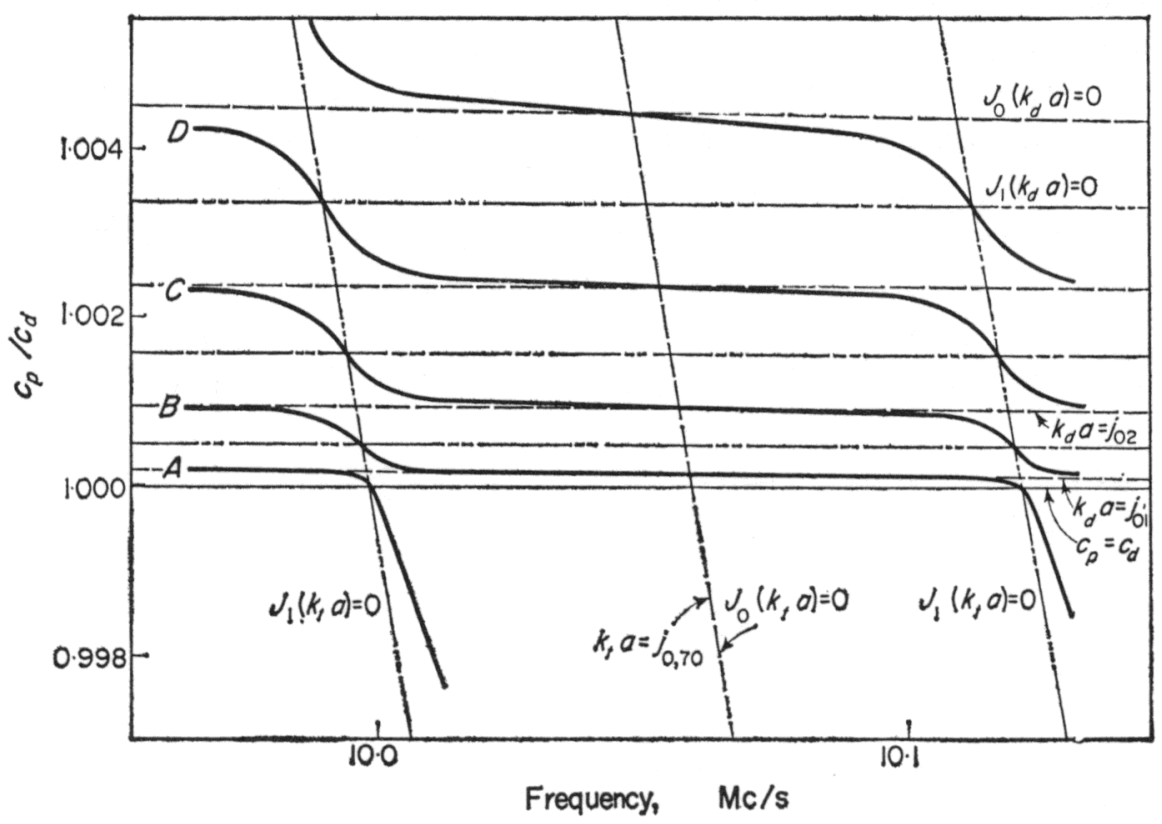

Fig. 12. Ratio of phase velocity, $c_{p}$, to longitudinal wave velocity, $c_{d}$, at high frequencies for nontorsional modes near $c_{p}=c_{d}$.

If we recall Eq. (37), we see that the expression for the potentials, and hence the displacements, are periodic in $z$, the axial direction. Thus, the previously plotted displacements and stresses specified no axial position - the same behavior obtained down the whole length of the rod, except for a sinusoidal variation. But when we realize that each mode has a different value of $\mathrm{k}_{0}$ and thus, for a given frequency, has a different space period, we can see that interference effects will determine the net or resultant particle movements due to all excited modes. If we were to attach a receiving crystal to the end of our rod, it is apparent that the signal generated at that point will depend on the length of the rod. This fact hampers accurate measurements of the intrinsic attenuation of the rod, for unless one can compute the effect on apparent attenuation due to the geometry of the rod, one can measure only changes in the intrinsic attenuation which do not involve changes in the rod's geometry. Measuring the change in attenuation versus temperature can be a risky thing, for instance, because the expansion of the rod will change the interference pattern at the receiver. If one mode only could be excited, then this problem would not arise; in general however, this is improbable except at low frequencies (kilocycle range).

Carome, Witting, and Fleury $(16,17)$ have illustrated this point very nicely. They made theoretical calculations of the integrated pressure over the face of the rod, integrating both amplitude and phase over all modes for 
a piston-type source, that is, a transducer vibrating by varying its thickness, attached to one end of the rod, and a like transducer receiving the signal at the other end (or the same transducer receiving an echo from the far end of the rod). They calculated the resulting signal as a function of path length for both solid rod boundary conditions and liquid rod boundary conditions. In the liquid boundary condition, the liquid is as sumed to be so rigidly confined that there can be no radial particle motion at the surface. Of course, in this case, the stresses will not be set equal to zero as was done in the previous discussion. To check their calculations, they set up a liquid column and sent sound from a transducer up the column, reflected it off the top surface, and received the echo. By varying the height of liquid, they could thus change the length of their "rod." It is interesting to note that their experimental results with the liquid column fit the theoretical curves for solid rods rather well, and did not match the liquid column theory at all. This happened because their liquid was confined in a tube, and the tube boundary conditions would match the solid rod theory much better than the rigidly confined liquid boundary theory. In Fig. 13, we see their theoretical curve for the case where the ratio of rod diameter to transducer diameter is 1.5. In this figure they plotted the apparent los s of sound intensity as a function of distance from the transducer. In Fig. 14 we see photographs of their oscilloscope traces. In each case, pulse amplitude increases in the downward direction of the photograph; thus, reading from the bottom of the photo up to the end of the pulses gives us a measure of loss; increasing distance from the bottom means greater intensity loss. The photographs were made by a time-exposure method. At any instant of time, one vertical line, representing an echo, was visible on the oscilloscope face. As the water level was raised in the tube, the pulse moved across the scope face, due to the increasing time it took the echo to return to the transducer. The camera was open during this whole process, so the resulting picture is a "continuous multiple exposure" of the individual pulses at each instant of time. We can compare Figs. 13 and 14 and see the similarities in structure. Figures 15 and 16 show a similar sequence for a ratio of diameters of two; Fig. 16 was taken at $1 \mathrm{mc} / \mathrm{sec}$.

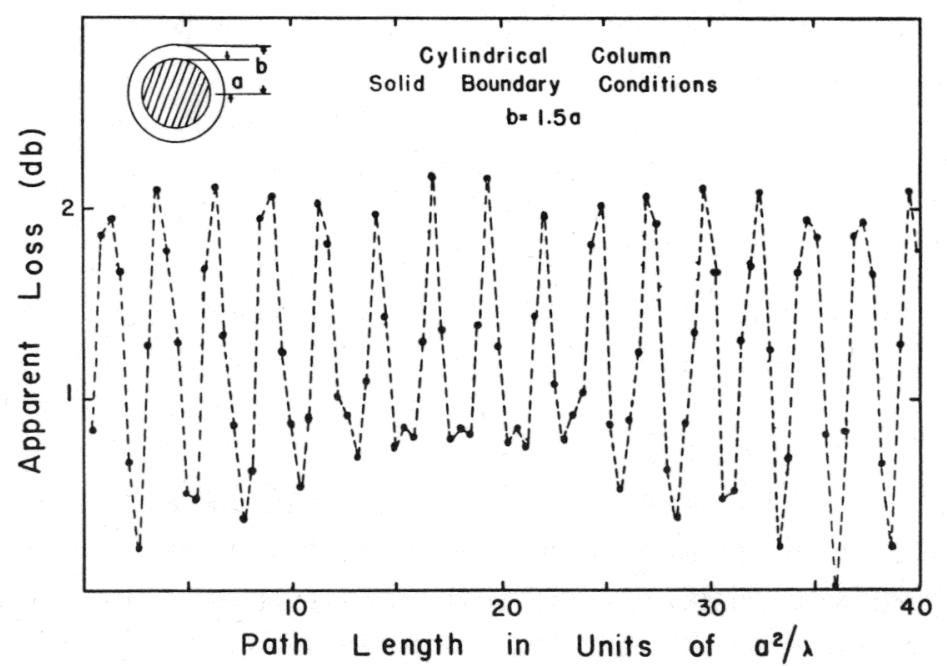

Fig. 13

Attenuation due to interference effects in waveguide as a function of distance along rod. Theoretical curve. 


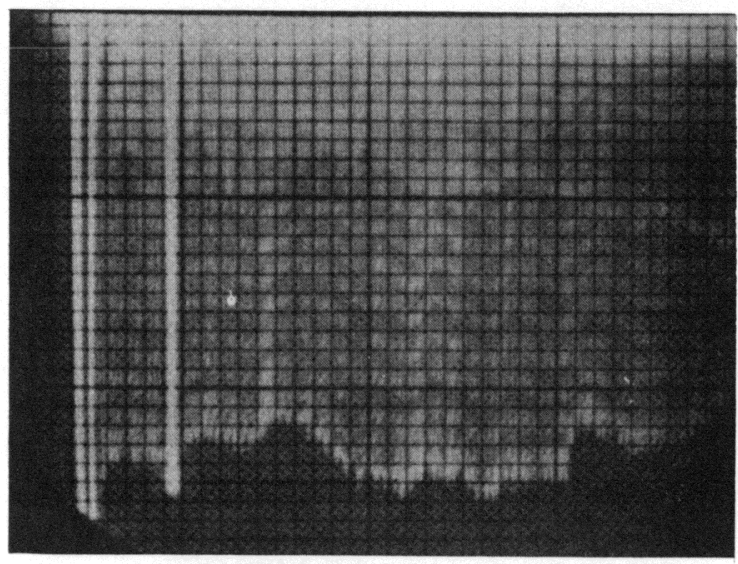

a) $1 \mathrm{Mc}$

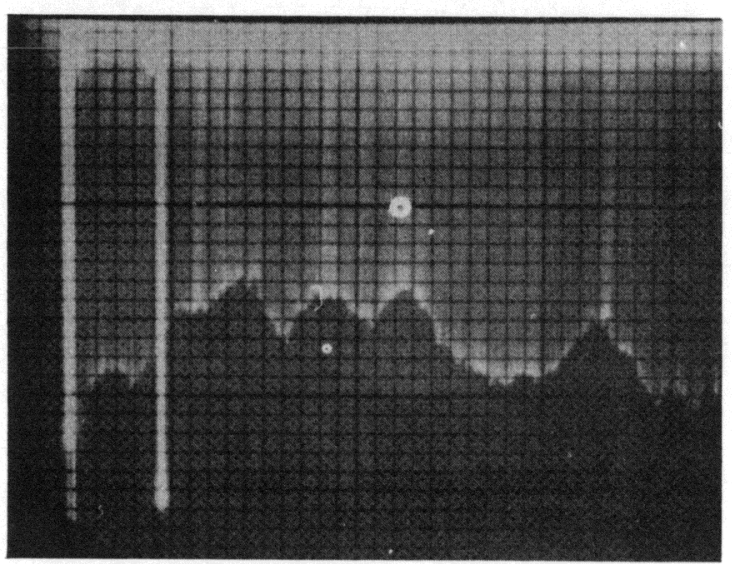

b) $3 \mathrm{Mc}$

Fig. 14. Oscillographs of attenuation as a function of water path length for ratio of column diameter to transducer diameter of 1.5 .

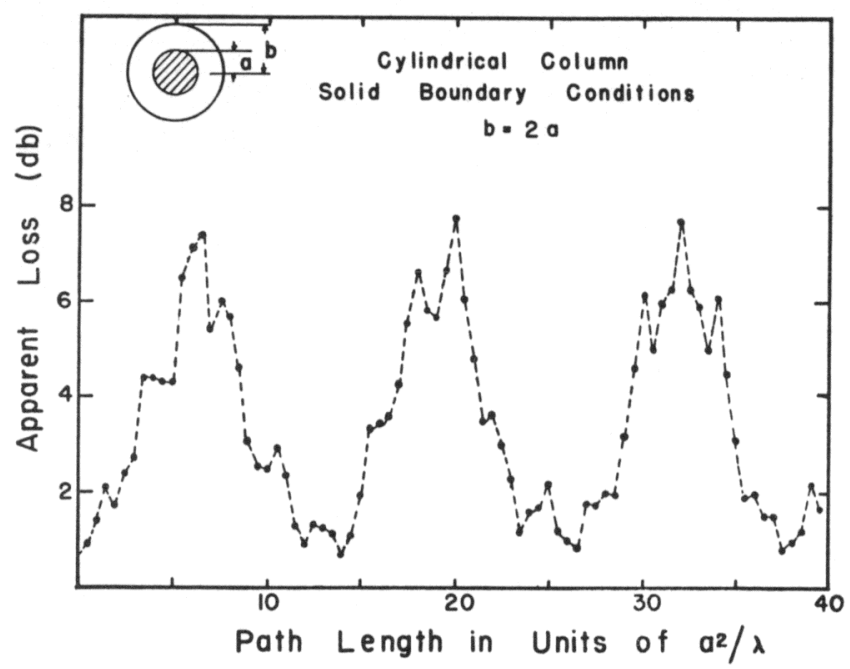

Fig. 15. Attenuation due to interference effects in waveguide as a function of distance along rod.

Theoretical curve.

Figure 17 is an illustration by Hüter(18) showing the effect on particle displacement of changing the ratio between transducer size to rod size.

There is good reason, on both theoretical and experimental grounds, to doubt the validity of the previous solutions when the sound introduced into the bar is in the form of a high-frequency pulse. The change in velocity with frequency does not show the expected plateau and steps, as we saw in Fig. 12.(19) Instead, the change appears to be a gradual one with no jumps or discontinuities. When a narrow-bandwidth rf pulse, that is, a pulse of radiofrequency oscillations containing at least 4 or 5 cycles, is introduced into a rod, a completely unpredicted phenomenon is observed. 


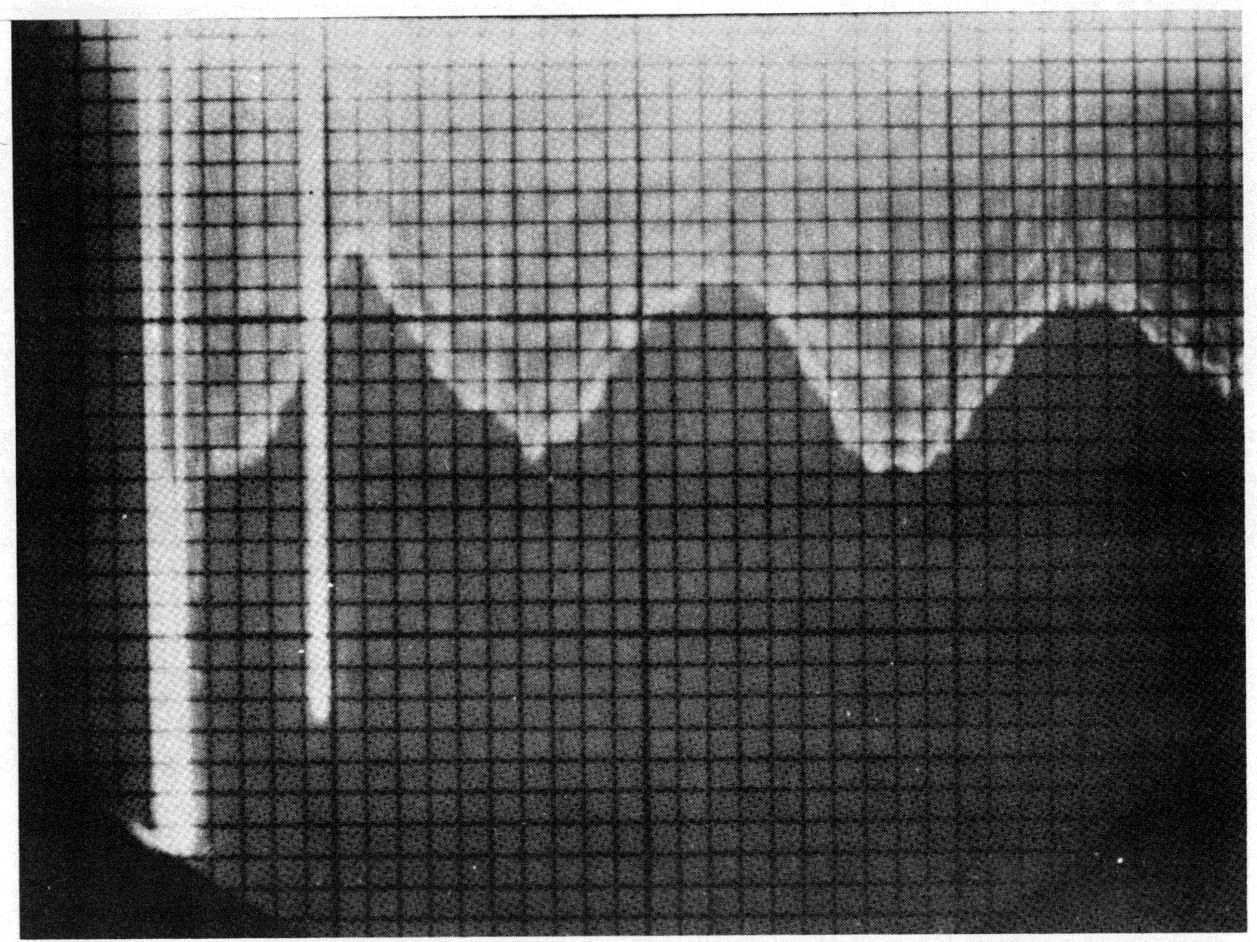

Fig. 16. Oscillographs of attenuation as a function of water path length for ratio of column diameter to transducer diameter of 2 . Taken at 1 megacycle.

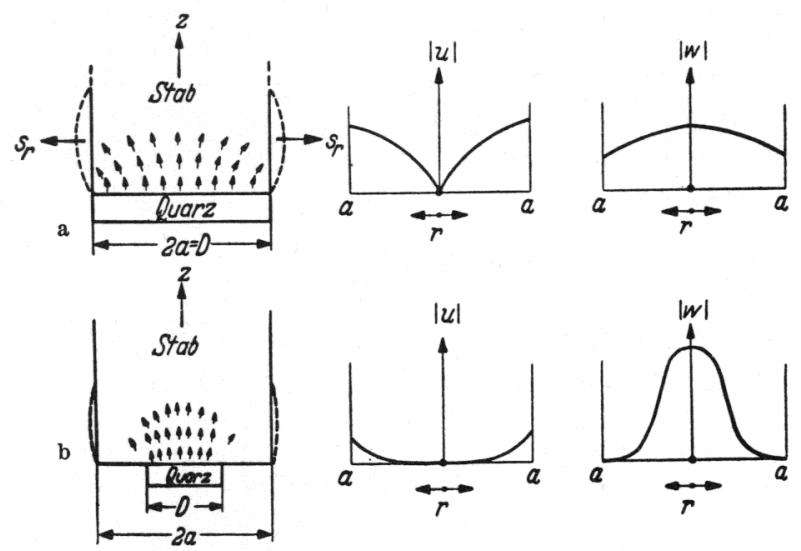

Fig. 17. Radial (u) and axial (w) displacements as a function of radial distance, $r$, for two ratios of transducer size to rod size.

The pulse travels down the rod at a velocity very close to $c_{d}$, and it is followed by secondary pulses, all separated by the same time interval $\Delta t$. To see how this may come about, consider Fig. 18 which is adapted from 
Redwood. (20) Here $L_{0}$ is a longitudinal wave incident at angle a d as discussed previously. Upon reflection from the radial boundary of the rod, a transverse wave $T_{1}$ and a longitudinal wave $L_{1}$ will be generated; the reflection angle for $L_{1}$ will be ad while the reflection angle for $T_{1}$ will be $a_{t}$, as given by Eqs. (36). Upon striking the opposite side of the rod, $\mathrm{T}_{1}$ generates a longitudinal wave $L_{2}$ and a transverse wave $T_{2}$. The velocity of a transverse wave is smaller than that for a longitudinal wave [Eqs. (1)-(4)]; in most metals, $L_{1}$ will reach plane $A-A$ in the figure before $T_{1}$ reaches that plane. Knowing the velocities $c_{d}, c_{t}$, and angles $a_{d}, a_{t}$, the time delay between these two events can be calculated to be

$$
\Delta t=\frac{2 a}{c_{t} \cos a_{t}}-\frac{2 a}{c_{d}} \tan a_{t}
$$

At high frequencies, $\mathrm{a}_{\mathrm{d}} \simeq 90^{\circ}$. Equation (40) is then approximately

$$
\Delta t \simeq 2 a \sqrt{\frac{l}{c_{t}^{2}}-\frac{1}{c_{d}^{2}}} \quad .
$$

These equations hold only if $c_{p}>c_{d}$.

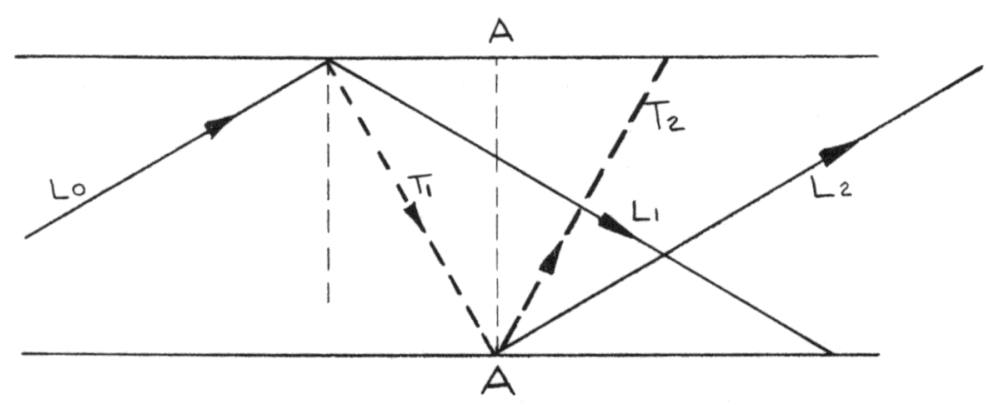

Fig. 18. Trailing Pulse Generation in a Rod

The Pochhammer-Chree frequency equations and the corresponding wave equation solutions make no provision for the foregoing observations. Redwood(2l) proposes a different type of solution in view of what we have just discussed, namely, that at every moment at every point in the bar there are not 4 waves to be considered. Redwood's solution is to provide for one incident wave, the initial longitudinal one $L_{0}$, and 2 reflected waves, $\mathrm{T}_{1}$ and $\mathrm{L}_{1}$. He therefore does not accept Eqs. (37) as solutions of (35), but instead uses the following solution:

$$
\begin{aligned}
& \phi=A J_{0}\left(k_{d} r\right) \exp \left[i\left(\omega t-k_{0} z\right)\right] \\
& R=B H_{0}^{(1)}\left(k_{t} r\right) \exp \left[i\left(\omega t-k_{0} z\right)\right],
\end{aligned}
$$

where $\mathrm{H}_{0}^{(1)}$ is the zeroth-order Hankel function. 
The new frequency equation becomes

$$
0=k_{0}^{2} \frac{k_{t} H_{0}^{(1)}\left(k_{t} a\right)}{H_{1}^{(1)}\left(k_{t a}\right)}-\frac{1}{2}\left(\frac{\omega}{c_{t}}\right)^{2} \frac{1}{a}+\left[\frac{1}{2}\left(\frac{\omega}{c_{t}}\right)^{2}-k_{0}^{2}\right]^{2} \frac{J_{0}\left(k_{d a}\right)}{k_{d J_{1}}\left(k_{d} a\right)} \text {. }
$$

At the moment, a computer program is being written at Argonne to solve this equation, and we had hoped to be able to present the results at this symposium. However, we were not able to finish the computation in time. An approximate solution valid at high frequencies is given by Redwood,(19) and is shown in Fig. 19. Notice that there is only a gradual variation of phase velocity with frequency, and no plateaus at all. It seems as though this modified solution may certainly be correct for narrow bandwidth pulses. In order to check out our computer results when they are available, we have made three steel specimens, all of the same length and material, but of different diameter, as seen in Fig. 20. Upon having a plot of group velocity versus frequency, we can try various combinations of frequency and rod diameter to see if the experimental data fit the plots.

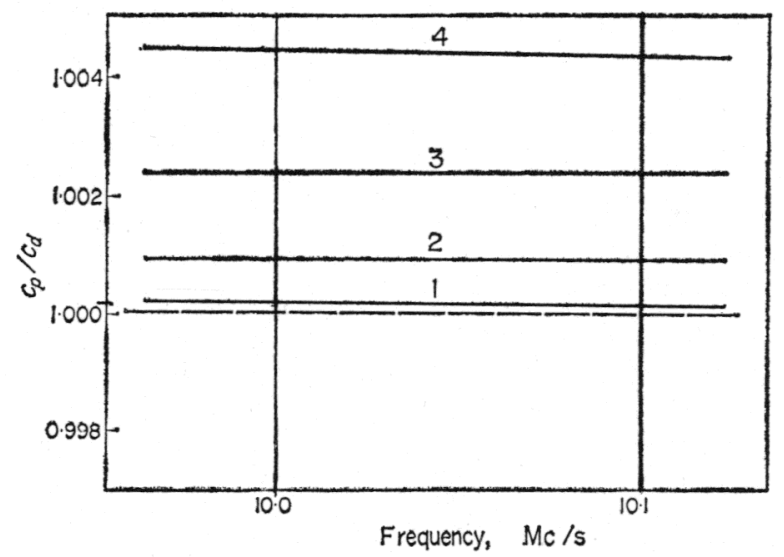

Fig. 19. Ratio of phase velocity, $\mathrm{c}_{\mathrm{p}}$, to longitudinal wave velocity, $\mathrm{c}_{\mathrm{d}}$, for high frequencies, for modified solution of wave equation. Modes near $c_{\mathrm{p}}=\mathrm{c}_{\mathrm{d}}$.

In Fig. 21 are shown some photographs taken of our oscilloscope trace. All 3 traces show the rf pulses which are transmitted through the steel specimens. In this setup, we have separate sending and receiving crystals. In the upper photo, we can see 6 main signals. The first and largest signal is the signal transmitted directly down the rod from the sending crystal to the receiver. The other large amplitude signals are echoes of the first; the sound has been reflected off of the receiving crystal, sent back down the rod, reflected off the transmitter end, and returned once more down the rod to the receiver. This is, of course, 
repeated for each succeeding echo. The attenuation of the sound as it travels through the rod can easily be seen. In between the main echoes the trailing pulses are visible.
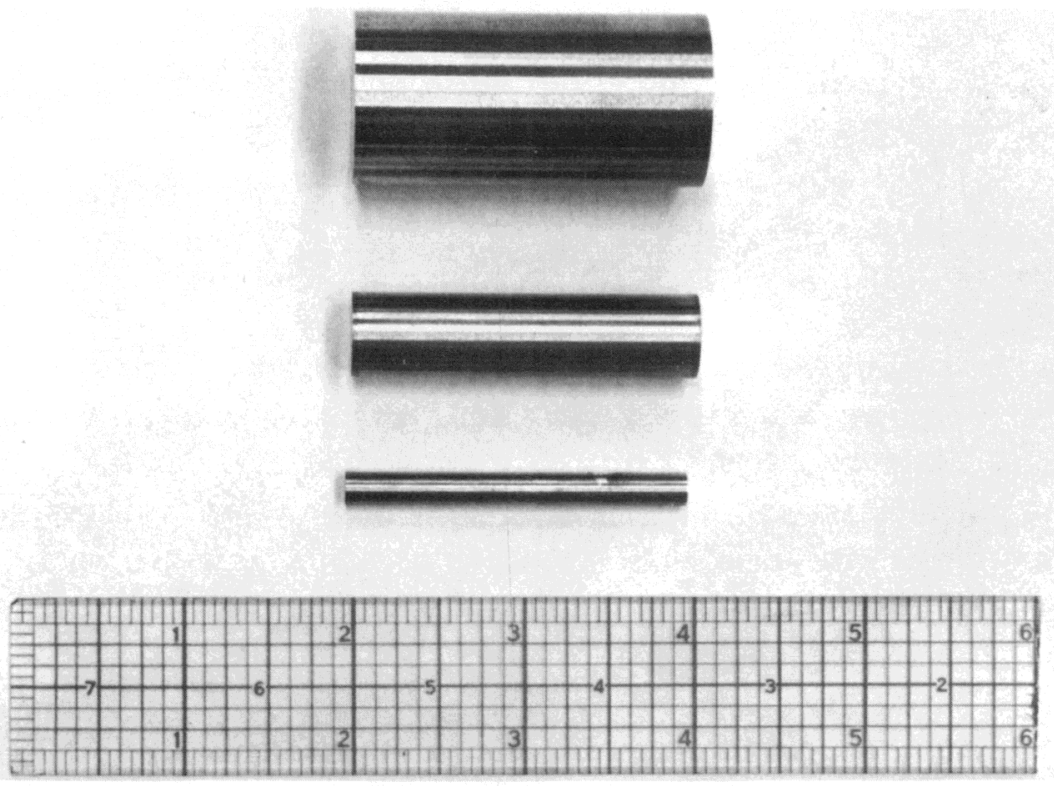

Fig. 20. Steel Specimens Used for Studying Mechanical Waveguide Effect

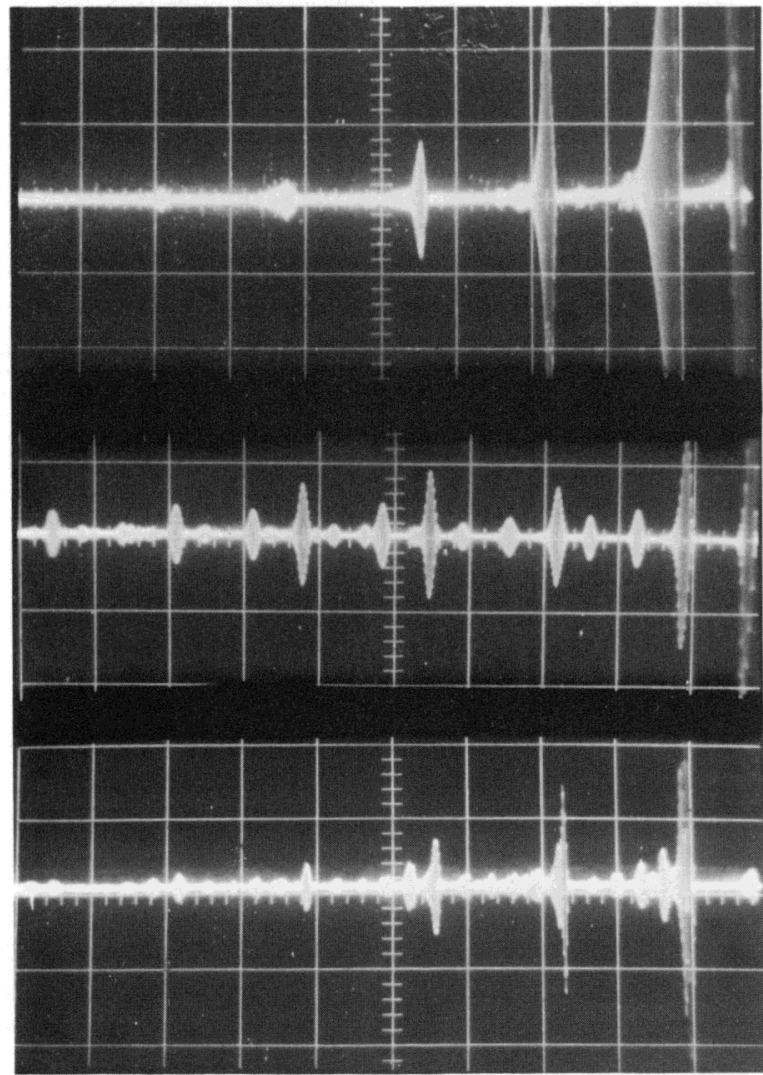

\section{Fig. 21}

Oscillographs of echoes observed in two steel specimens. Top photograph: one half inch diameter, $10 \mathrm{mc} / \mathrm{sec}$. Middle: one inch diameter, $5 \mathrm{mc} / \mathrm{sec}$. Bottom: one inch diameter, $15 \mathrm{mc} / \mathrm{sec}$. 
Compare the upper and middle photographs: the top one shows a $10-\mathrm{Mc}$ pulse propagating in the 0.5 -in.-diameter specimen; the middle picture shows a 5-Mc pulse in the one-inch rod; both were taken at the same sweep speed. For both pictures, the ratio $a / \Lambda$ is the same. The point to be noticed, however, is that the trailing pulses are different in the 2 photographs. Consider the space between the first 2 main echoes in both photographs. In the upper photo, there are 4 trailing pulses in that space, whereas only 2 appear in the middle photo. This is in good agreement with Eq. (41), which shows that the time between trailing pulses is proportional to the rod radius.

The bottom photograph was taken at 15 megacycles on the same specimen as was the middle photo. Although the change in attenuation with frequency shows clearly, this is not a geometrical effect but an intrinsic one. The main pulse and the trailing pulses have been somewhat distorted, but no significant change in signal velocity is apparent from these photos. Any velocity change with frequency must be very small, and must be measured by very accurate time-delay circuits. This has not yet been done.

Another experiment in which we are interested at the present time involves a determination of the elastic constants of several metals as a function of temperature. In these experiments, we use a bar with a specially shaped end, really a bar with a little bar on one end. This type of specimen is illustrated in Fig. 22. A quartz transducer is placed on the end of the bar without the extension; sound is then sent into the specimen, is first reflected from the shoulder, and then from the extreme end of the extension. Two echoes are thus received at the transducer. The sample is placed into a vacuum furnace shown in Fig. 23, with the transducer end outside, sticking out of the top of the furnace, while the extension is in the hot zone of the furnace. The associated electronic gear for generating and detecting the sound can be seen also. We are, of course, interested in what is going on in the extension, and therefore we are particularly interested in observing the 2 echoes from the boundaries of the extension.

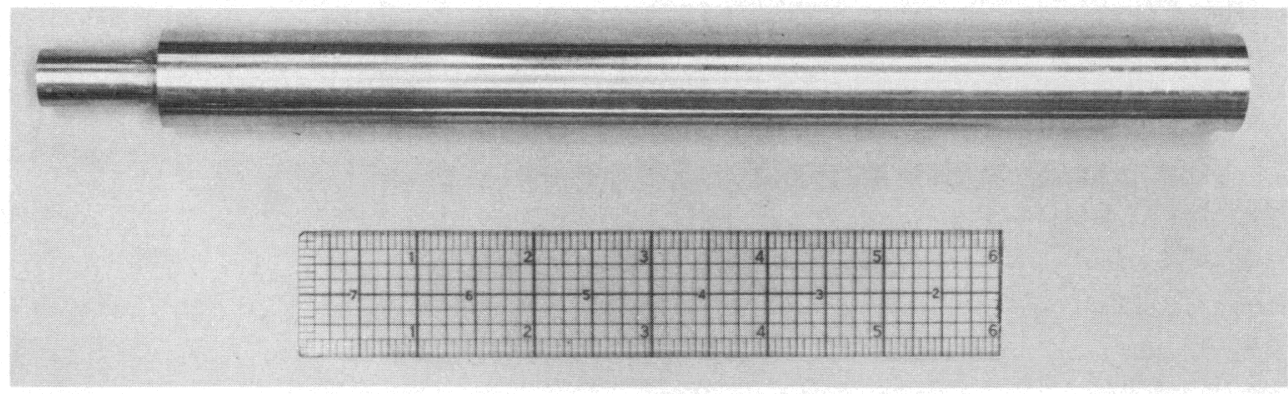

Fig. 22. Aluminum Specimen Used for Measurement of Elastic Constants Versus Temperature 


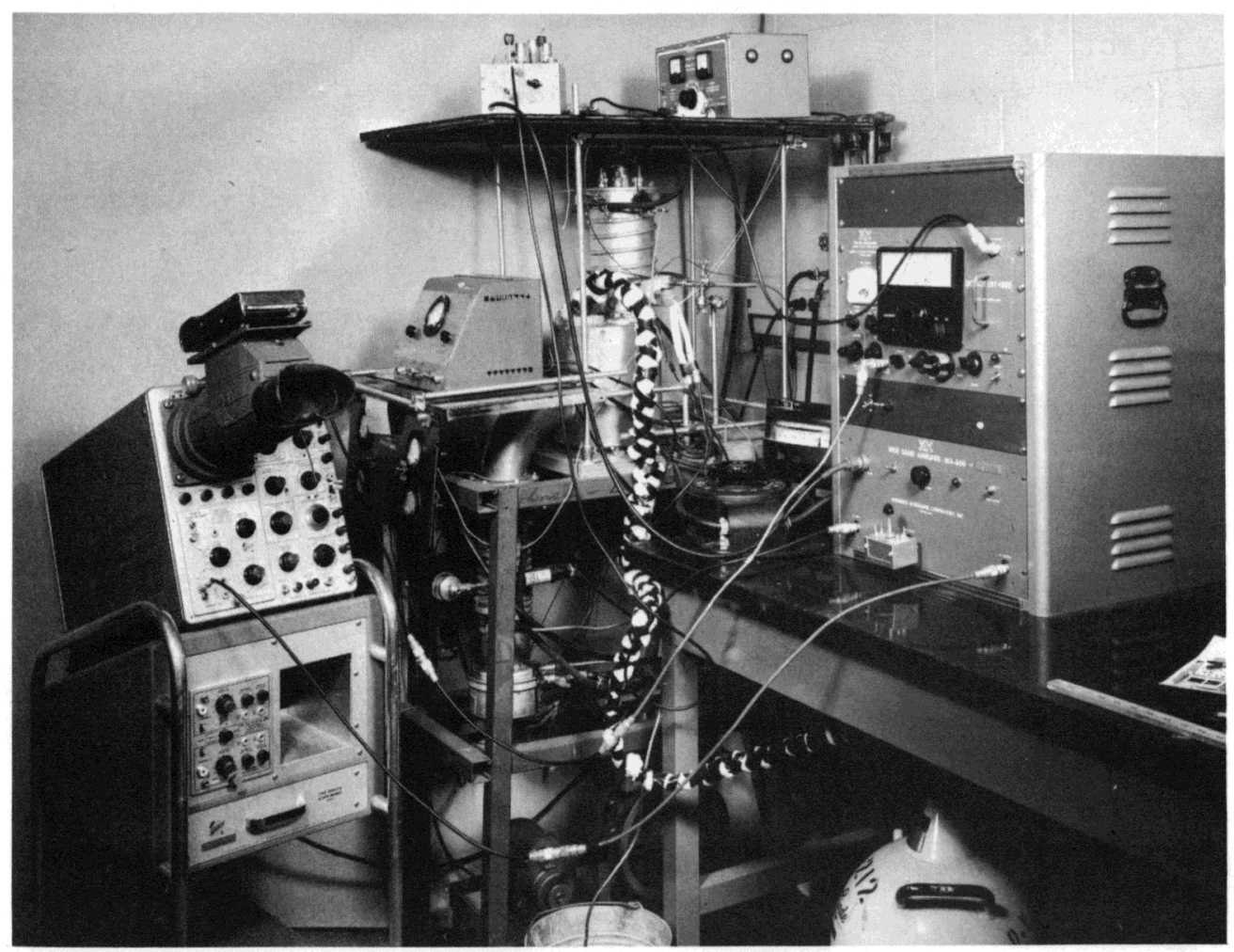

Fig. 23. Laboratory equipment used in studying variation of elastic moduli with temperature.

It so happens that, for the size of specimens that are useful in a practical experiment, the time between the receipt of the 2 echoes is of the same order of magnitude as the delay between the trailing pulses produced in the rod. If one is not able to identify accurately each pulse received, then it is impossible to interpret the experimental results. This is the major reason we have been led to the study of these waveguide effects in bars.

In Fig. 24 we see an oscilloscope trace of the sound in the aluminum bar at $15 \mathrm{Mc} / \mathrm{sec}$. The first 2 main echoes are shown along with their retinue of trailing pulses. The first pulse is the reflection from the shoulder; the second pulse is the first delayed pulse reflecting from the shoulder. The next pulse is the echo from the far end of the extension. At the end of this large pulse, the second delayed pulse reflecting from the shoulder starts. The next pulse seen is probably the echo of the first delayed pulse from the far end of the extension. The next pulses are not as easily identified. The identifications were made by means of the commonly accepted values of $\mathrm{c}_{\mathrm{d}}$ and $\mathrm{c}_{t}$ for aluminum and using Eq. (4l) to compute $\Delta t$. These identifications are not positive, and won't be until the numerical data is received from the Computer Group. 


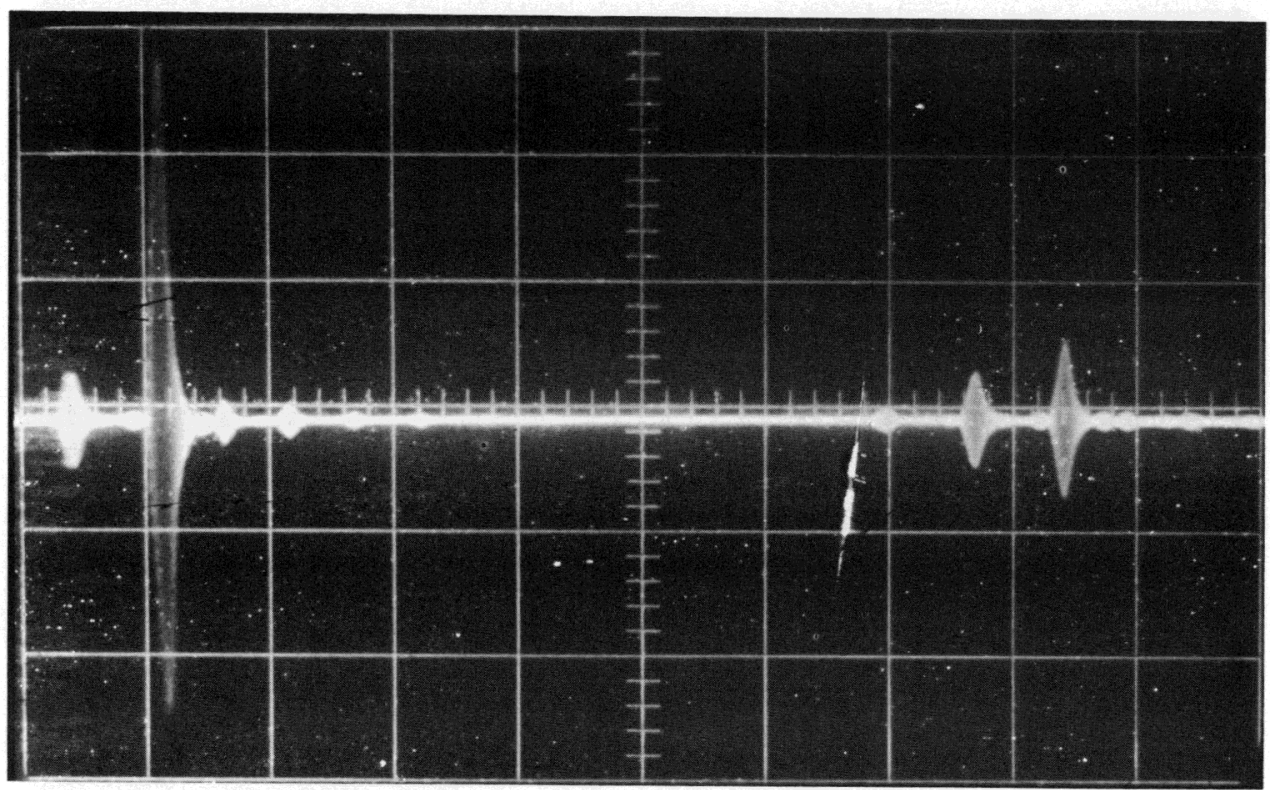

Fig. 24. Oscillograph of echoes in aluminum rod shown in Fig. 22. Frequency is $15 \mathrm{mc} / \mathrm{sec}$.

If it should happen that we are working in a region where $\mathrm{c}_{\mathrm{g}}$ is significantly different from $c_{d}$, and, not knowing this, use our measured value of $\mathrm{c}_{\mathrm{g}}$ as an approximation for $\mathrm{c}_{\mathrm{d}}$ in order to compute the elastic moduli, then obviously our modulus data will be significantly in error. Without the computer data on the frequency equation, we can never be sure of our results.

\section{Acknowledgements}

I would like to take this opportunity to acknowledge the help of the Nondestructive Testing Group at Argonne and to especially thank Dr. W. J. McGonnagle for his help and encouragement, and John G. Morgan for his invaluable technical assistance.

\section{$\underline{\text { References }}$}

1. Davies, R. M., "Stress Waves in Solids," Surveys in Mechanics, edited by Batchelor and Davies, Cambridge University Press (1956), pp. $64-138$.

2. Levitt, A. P., and A. G. Martin, Ultrasonic Determination of Elastic Constants of Metals at Elevated Temperatures, Watertown Arsenal Laboratories Technical Report No. WAL TR 143/34 (Sept 1959).

3. Truell, R., "Ultrasonic Attenuation and the Physics of Solids," Symposium on Physics and Nondestructive Testing, October 4 and 5, 1960, ANL-6346, pp. 109-126. 
4. Mason, W. P., Physical Acoustics and the Properties of Solids, Van Nostrand, Princeton, N. J. (1958) pp. 368-373.

5. Davies, op. cit. pp. 76-82.

6. Davies, R. M., A Critical Study of the Hopkinson Pressure Bar, Phil. Trans. Roy. Soc. A240, 375 (1948).

7. Kolsky, H., Stress Waves in Solids, Clarendon Press, Oxford (1953) pp. 87-98.

8. Redwood, M., Mechanical Waveguides, Pergamon Press, New York (1960).

9. Pochhammer, L., J. Reine Angew, Math. 81, 324 (1876).

10. Chree, C., Trans. Camb. Phil. Soc. 14, 250 (1889).

11. Redwood, op. cit. p. 149.

12. Kolsky, op. cit. p. 54.

13. Bancroft, D., Phys. Rev. 59, 588 (1941).

14. McSkimin, H. J., J. Acoust. Soc. Amer. 28, 484 (1956).

15. Redwood, op. cit. p. 142 .

16. Carome, S., and J. Witting, Theory of Ultrasonic Attenuation in Cylindrical and Rectangular Waveguides, Office of Naval Research Contract NONR2577(01), Project NR-384-309, Tech. Rept. 3, Dept. of Physics, John Carroll University, March 31, 1960.

17. Carome, S., J. Witting, and P. Fleury, Experimental Study of Diffraction and Waveguide Effects in Ultrasonic Attenuation Measurements, Office of Naval Research Contract NONR2577(01), Project NR-384-309, Tech. Rept. 4, Dept. of Physics, John Carroll University, November 30 , 1960.

18. Hüter, T., Z. fur Angew, Physik 1, 274 (1949).

19. Redwood, op. cit. p. 200.

20. Ibid, p. 192 .

21. Ibid, p. 197.

\section{References Not Cited in Text}

22. Abramson, H., J. Acoust. Soc. Amer. 29, 42 (1957).

23. Abramson, H., H. Plass, and E. Ripperger, Advances in Applied Mechanics, V., Academic Press (1958) p. 111 .

24. Adem, J., Quart. Appl. Maths. 12, 261 (1954). 
25. Davies, R., Appl. Mech. Revs. 6, 1, (1953).

26. Field, G., Canadian J. Research 5, 131 (1931).

27. Field, G., Canadian J. Research 5, 619 (1931).

28. Green, W., Article in Progress in Solid Mechanics, Volume I, edited by Sneddon and Hill; North Holland Publishing Co. (1960).

29. Holden, A., Bell Syst. Tech. J., 30, 956 (1951).

30. Hsieh, D., and H. Kolsky, An Experimental Study of Pulse Propagation in Elastic Cylinders, Brown U. Tech. Report 4, October 1957, ASTIA 147189.

31. Hudson, G., Phys. Rev., 63, 46 (1943).

32. Hughes, D., W. Pondrom, and R. Mims, Phys. Rev. 75, 1552 (1949).

33. Kolsky, H., Phil. Mag., 45, 712 (1954).

34. McNivin, H., J. Acoust. Soc. Amer. 33, 23 (1961).

35. Meitzler, A., I.R.E. Convention Record, Part 9, March 19-22, 1956, p. 55 .

36. J. Acoust. Soc. Amer. 33, (1961) p. 435

37. J. Acoust. Soc. Amer. 33, (1961) p. 856

38. Mindlin, R., and E. Fox, J. Appl. Mech. 27, 152 (1960).

39. Mindlin, R., and H. McNivin, J. Appl.Mech。27, 145 (1960).

40. Oliver, J., J. Acoust. Soc. Amer. 29, 189 (1957).

41. Pao, Y., Dispersion of Flexural Waves in an Elastic Circular Cylinder, Part II, Office of Naval Research Report NR-064-388-TR-39, Columbia University, November, 1960.

42. Pao, Y., and R. Mindlin, J. Appl. Mech. 27, 513 (1960).

43. Redwood, M., Proc. Phys. Soc. 70, 721 (1957).

44. Proc. Phys. Soc. $\underline{72}, 841$ (1958).

45. J. Acoust. Soc. Amer., 31, 442 (1959).

46. Redwood, M., and J. Lamb, Proc. Phys. Soc. B70, 136 (1957).

47. Ruedy, R., Canadian J. Research 5, 149 (1931).

48. Sherwood, J., J. Acoust. Soc. Amer., 30, 979 (1958).

49. Tefft, W., J. Res. Nat'l. Bur. Standards, 64B, 237 (1960). 


\section{Discussion}

\section{MECHANICAL VIBRATIONS IN SOLID RODS}

F. R. Rollins, Midwest Research Institute: During the discussion on temperature effects of elastic moduli, I wondered how you determined the variation in the distance between the shoulder and the end of the rod, and corrected for this extension with temperature.

R. G. Peterson: I'll have to use the standard data for the thermal expansion coefficient.

F. R. Rollins: You're not measuring that quantity.

R. G. Peterson: That's correct. 
by

Robert J. Maurer

Physics Department

University of Illinois

Urbana, Illinois

\begin{abstract}
Optical absorption due to impurities and certain types of lattice defects is a sensitive method for their identification and quantitative determination. Some examples of recent studies of the hydroxyl ion, the bromide ion, and anion vacancies in potassium chloride crystals are discussed. The dc and ac conductivity of ionic crystals can be used to reveal the presence of impurities. Recent results of zone refining the silver and alklai halides are presented. The use of photoconductivity and certain magnetic properties of crystals as a means of determining purity and perfection is reviewed.
\end{abstract}

Due to illness, Dr. Maurer was unable to present a paper. 


\author{
INFRARED PHYSICS \\ by \\ H. L. Sachs \\ Perkin-Elmer Corporation \\ Norwalk, Connecticut
}

\begin{abstract}
Continuous, remote measurement of the radiant emis sion from heated surfaces offers the capability for detection of flaws or voids internal to a specimen. The buildup of sur face temperature gradient and departure from norm in the vicinity of internal flaw regions is discussed with emphasis on laminated structures and defects in metal to thermal insulator bonds.

The performance requirements for infrared instrumentation with measurement capabilities suited for this task are outlined and special attention given to practical aspects of the problem. An experimental technique and some recent promising results are presented.
\end{abstract}

\title{
I. Introduction
}

A high level of current interest centers on the application of infraredemission measurements for inspection of bulk materials. Development techniques for detection of thermal emission from test materials at temperatures well below incandescence $\left(60^{\circ} \mathrm{C}\right.$ to $\left.400^{\circ} \mathrm{C}\right)$ offers promising possibilities for surface-roughness gauging, dimensional gauging, temperature contouring and nondestructive internal inspection of various structures. Perkin-Elmer is presently engaged in a Thermal-IR program directed to the detection and location of internal defects in solid propellant missile casings, characterized by unbondedness and voids at the insulating liner interfaces.

This presentation serves to direct attention to some of the key IR system elements and techniques available for application, and briefly reviews initial results with the Thermal Infrared Inspection System (TIRI).

\section{Techniques}

The infrared region is conventionally divided into 3 bands of varying . wavelength extent, as indicated in Fig. 1. The techniques and problems associated with measurement in each of these regions differ markedly, so that this segregation is quite sound. The near infrared region extending 
from 0.7 to $2.5 \mu$ is the most thoroughly exploited. Pyrometry normally involves measurements of high-temperature surfaces at or near incandescence. Conventional optical glass materials are quite suitable for window and lens elements, and detectors for this region are readily available, i.e., phototubes, photodiodes, and lead sulphide.

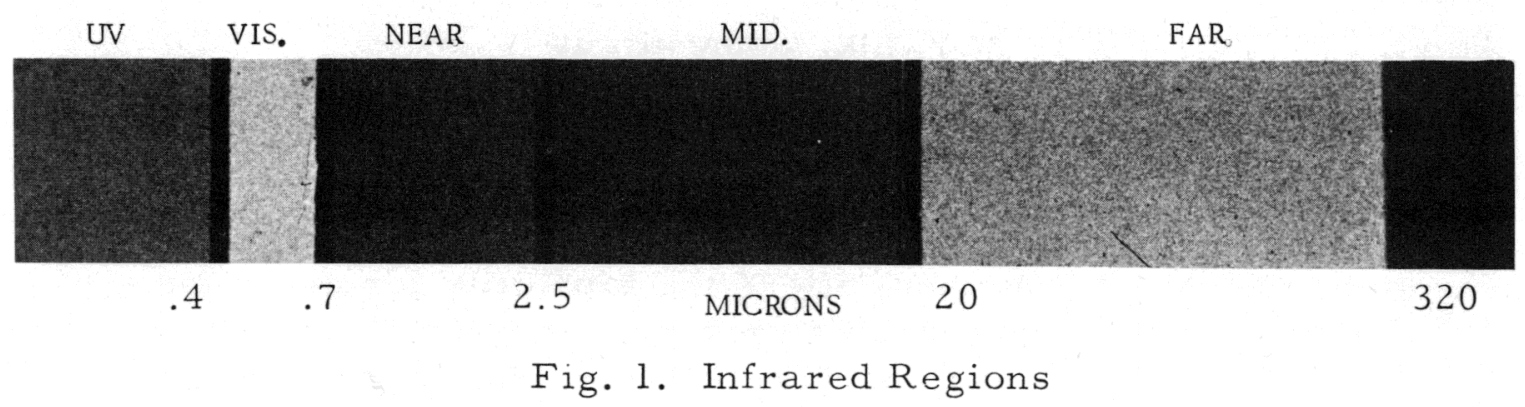

The median infrared region extending from 2.5 to $20 \mu$ has been intensely exploited for transmission spectroscopy, and lately for emission spectroscopy and radiometry of surfaces at lower temperatures. Below $500^{\circ} \mathrm{K}$, this band offers the highest emission levels and signal-contrast capabilities.

The region is characterized by atmospheric absorption bands at $2.6-2.9,4.1-4.5,5.2-7.5$ and beyond $14 \mu$ due to water vapor and $\mathrm{CO}_{2}$. The absorption properties are strong enough to limit severely the received radiation, even over path lengths as short as $10 \mathrm{ft}$. The least affected of the "windows" between these bands extends from 7.5 to $14 \mu$ and offers the greatest potential for low-temperature emission pyrometry.

Thermal detectors, thermistor bolometers, Golay cells, and thermocouples have been traditionally used for many years. Within the past 10 years, however, photoconductive devices have been employed with success. Their intrinsic sensitivity and speed of response are in many cases an order of magnitude greater than corresponding values for thermal units, and these capabilities are required for special applications. Generally, they must be operated at temperatures from $-80^{\circ} \mathrm{C}$ to $-196^{\circ} \mathrm{C}$ for their photoconductive mechanism to be effective. Pnotoconductors with long-wavelength $(14-20 \mu)$ response require liquid helium cryogenics to $2-4^{\circ} \mathrm{K}$.

The extreme infrared region from 20 to $320 \mu$ and beyond, currently being investigated for special plasma pyrometry problems, is perhaps the most challenging and demanding in technique and devices. Only recently have photoconductive detectors become available that bridge the gap to microwave frequencies in the millimeter and submillimeter region. 
Planckian "black body" emission characteristics into an environment of $0^{\circ} \mathrm{K}$ are shown in Fig. 2 for temperatures ranging from 300 to $600^{\circ} \mathrm{K}$. The wavelength of peak emission for the continuum form the locus of a line described by the Eq. $\lambda_{p}=2940 \mathrm{X}\left(\mathrm{T}^{\circ} \mathrm{K}\right)$.

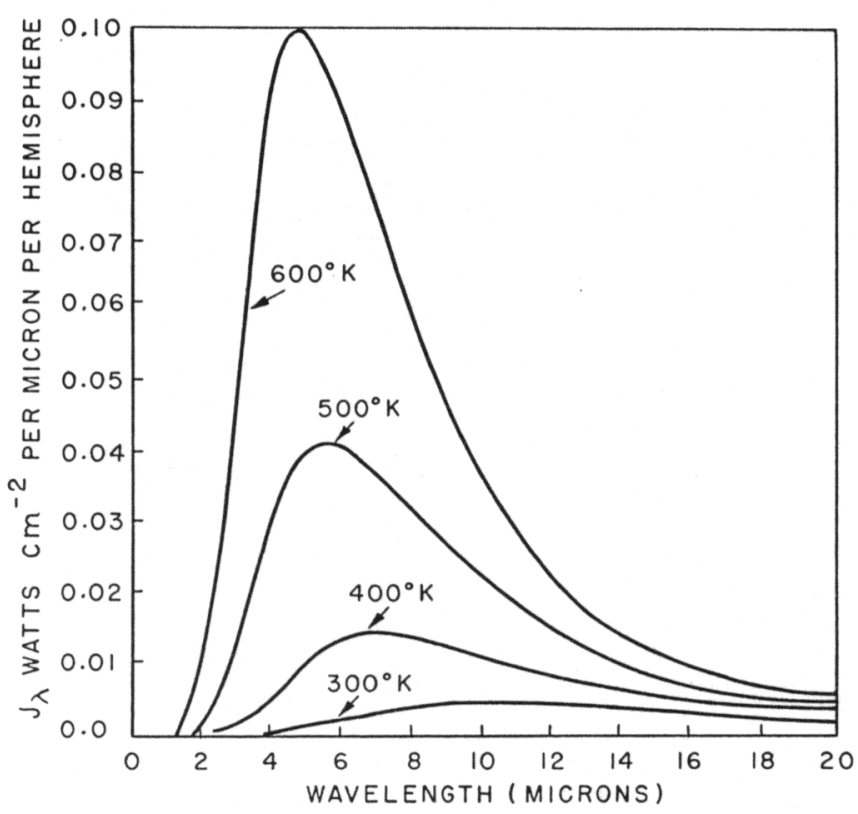

Fig. 2

Planckian Emission

Another property of interest, common to all distributions, shows $70 \%$ of the emitted energy into a hemisphere at $0^{\circ} \mathrm{K}$ will lie in the spectral range from $0.5 \lambda_{\mathrm{p}}$ to $2 \lambda_{\mathrm{p}}$.

In most problems of surface measurement, the ideal emitter characteristic of Fig. 2 is rarely observed. Generally, heated surfaces exhibit a Planckian distribution over limited, but fortunately significant, wavelength regions characterized by "grey body" emissivities from 0.05 to 0.9. The surface roughness of plates and the surface material strongly affect the wavelength-dependent emissivity factor. For example, 100-microinch-finish bare aluminum exhibits an emissivity of about 0.30 at $2.0 \mu$ falling to 0.05 at about $8 \mu$. Polished glass, on the other hand, exhibits an emis sivity greater than 0.90 at $8.0 \mu$.

Instrumentation for the remote measurement of infrared surface emission has progressed to an advanced and sophisticated state primarily because of significant advances in basic components.

Figure 3 illustrates the very simplest system of radiation detection. Radiation from the target is collected and focussed by a lens or mirror element onto a suitable detector. By means of motion of secondary optical elements within this system, the surface of an object can be rapidly scanned. 


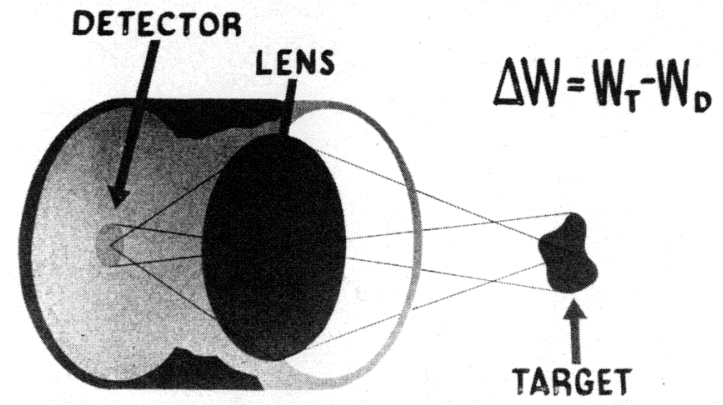

Fig. 3. Basic Detection System
The sequence of signal information derived from the detector can be presented as a remote pictorial display of the surface under study. In some cases, the surface may be moved in a regular manner while the optical system remains stationary.

\section{A. Detectors}

The detector element generally is the key to best performance, and the type selected is initially dictated by spectral region, speed of response, and ease of operation. Figure 4 provides a tabulation of present devices. The left-hand column indicates the temperature at which the detector is normally operated. In Fig. 5 is a plot of the characteristic spectral responsibilities of several important types, and there is indicated the level of sensitivity ( $\left.D^{*}\right)$ that can be expected with units of high quality.

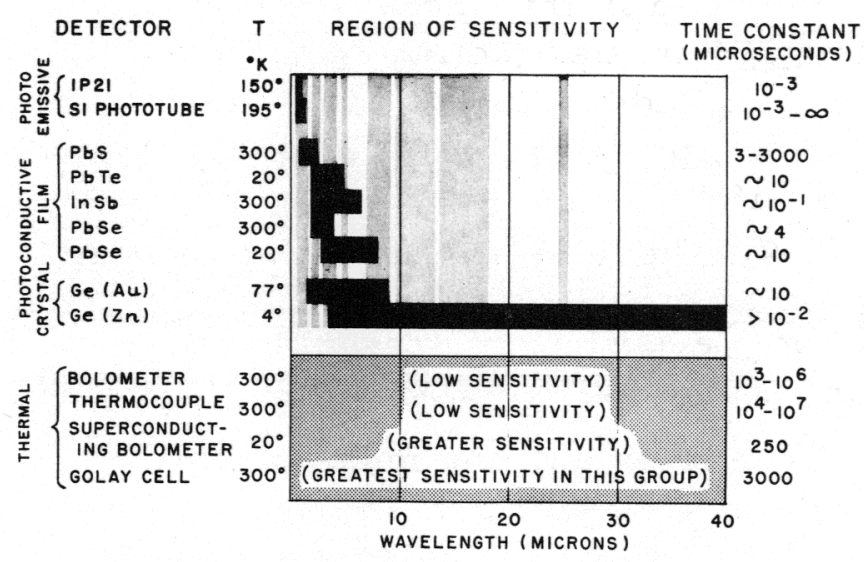

Fig. 4

Tabulation of IR Detectors

COMPARISON OF TWELVE RADIATION DETECTORS

Fig. 5

Spectral Response of Detectors

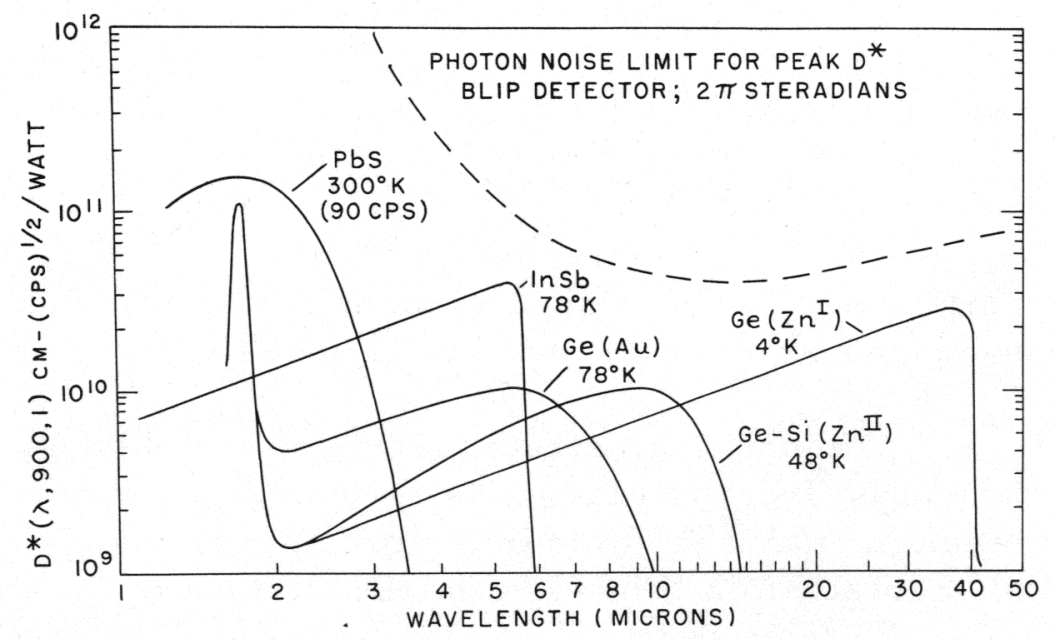


Lead sulphide with a peak spectral sensitivity between 2.3 and $2.7 \mu$ is often operated at dry ice temperature, which enhances the sensitivity and extends the useful wavelength of response.

Between 2.7 and $7 \mu$, a range of lead salt detectors are available that exhibit excellent sensitivity throughout this spectral region. Lead selenide (not shown) is currently in wide use and is available with a sensitivity or $D^{*}$ of about $2 \times 10^{10}$ at $6.5 \mu$. These detectors are thin-film, current-biased photoconductors, and absorption of radiation is primarily a surface phenomena.

Intermetallic photoconductive devices have come into dominant usage in the past several years. Indium antimonide, is typical of this group. When cooled to liquid nitrogen temperature, $-196^{\circ} \mathrm{C}$, the best detectors of this type exhibit a $D^{*}$ of $6 \times 10^{10}$ at $5.0 \mu$.

Indium antimonide can be fabricated to favor operation in one of several modes. With the establishment of a junction just under the surface, the detector exhibits a photovoltage response analogous to a silicon solar cell. The detector is inherently self-biased in this mode. Signal output and noise are intrinsically low, generally in the fractional-microvolt region. With other means of fabrication, indium antimonide can be operated as a current-biased photoconductor, providing much higher intrinsic noise and signal levels with little sacrifice in sensitivity. The photoconductive mode and a photomagnetic mode, the latter achieved with a magnetic field "bias," permit operation of specially fabricated types at room temperature with wavelength sensitivity extending to $7.5 \mu$. Uncooled, their sensitivity is about of the same order as for bolometers or thermocouples $\left(D^{*} \sim 10^{8}\right)$. Response time, however, is faster by orders of magnitude, characteristically less than $0.1 \mu$ sec.

The development of a unique indium antimonide detector sensitive at wavelengths to a $1000 \mu$ has recently been reported.** The unit is cooled with liquid helium and, under these conditions, exhibits a sensitivity close to that of lead sulphide. Microwave klystron sources are employed for certain measurements with this device.

Bulk germanium detectors, with carefully controlled impurity "doping," constitute an important class of devices for special applications in the region from 8 to $40 \mu$. In addition to the gold-and zinc-doped units shown, mercury and antimony doping makes available cutoffs at 14 and $20 \mu$. These detectors require cooling from $-196^{\circ} \mathrm{C}$ to liquid helium temperatures, depending on cutoff wavelength.

**Impurity photoconductivity in n-type InSb, E. H. Putley, R.R.E. 
For a wide range of applications, where ease of operation is essential, the thermistor detector, a thin, sintered oxide flake thermally sensitive to radiation, offers a $D^{*} \sim 10^{8}$, and typical response time of one millisecond at room temperature. Its flat responsivity to wavelengths from 1 to beyond $20 \mu$ is another important factor.

\section{B. Spectral Isolation}

One of the major problems confronting the instrument designer requires the isolation of the spectral window in which the radiation measurement can best be made, thus eliminating effects due to spurious reflection and background radiation. Recent developments in infrared filter techniques for the median infrared are shown in Fig. 6, a composite grouping of longwavelength multilayer elements fabricated here. The high degree of control of short-wavelength cutoff permits precise selection of characteristic in the range from 4 to $12 \mu$. Also shown, is a 3-4- $\mu$ band-pass element characteristic of filters available in the range from 0.70 to $7.0 \mu$. Total transmission in the "blocked" or attenuation regions is held below $0.025 \%$, while pass band transmittance is typically greater than $70 \%$.

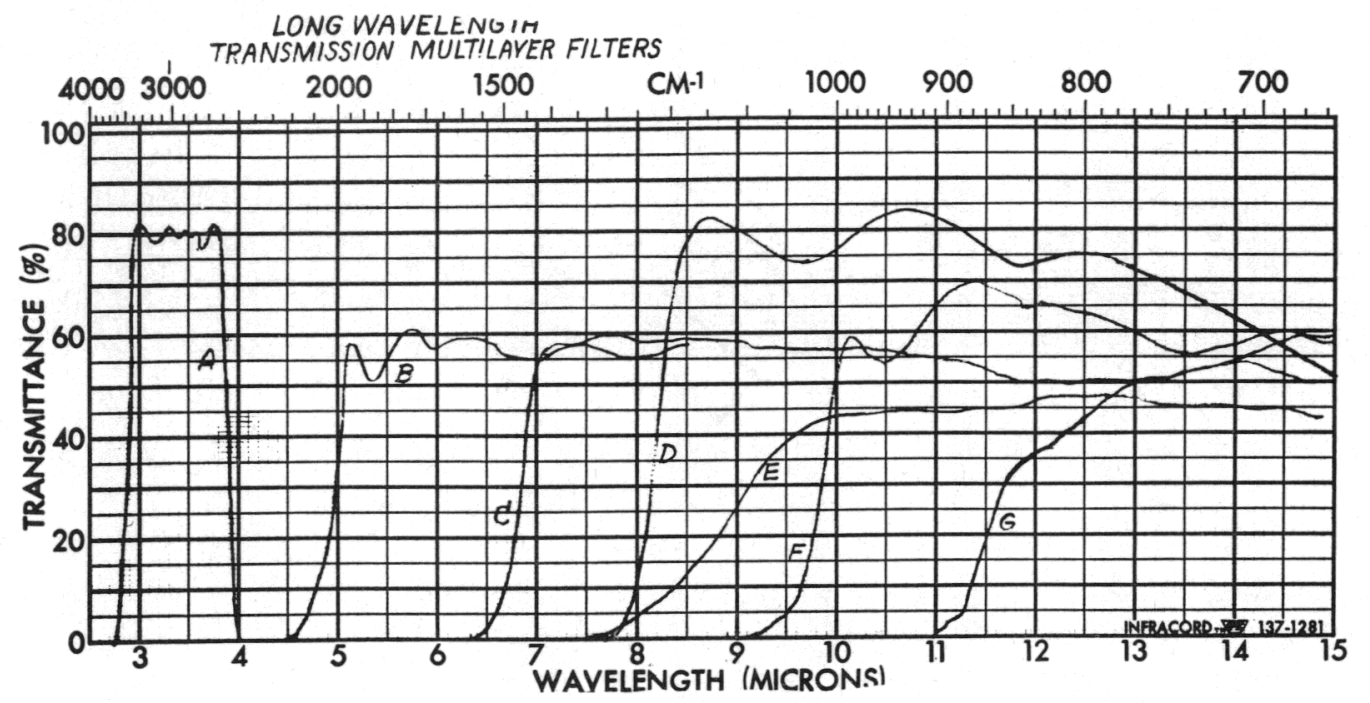

Fig. 6. IR Isolation Filters

C. Devices

Figures 7 through 10 illustrate several practical applications for the basic detection system. The Hot Box Detector, a trackside monitor developed by the Servo Corporation of Ameria, detects overheated journal bearings in passing freight cars. The thermistor detector unit is planted and focussed so that radiation from the journal box is optimized. The resultant signal is relayed to a central station for evaluation and decision. 


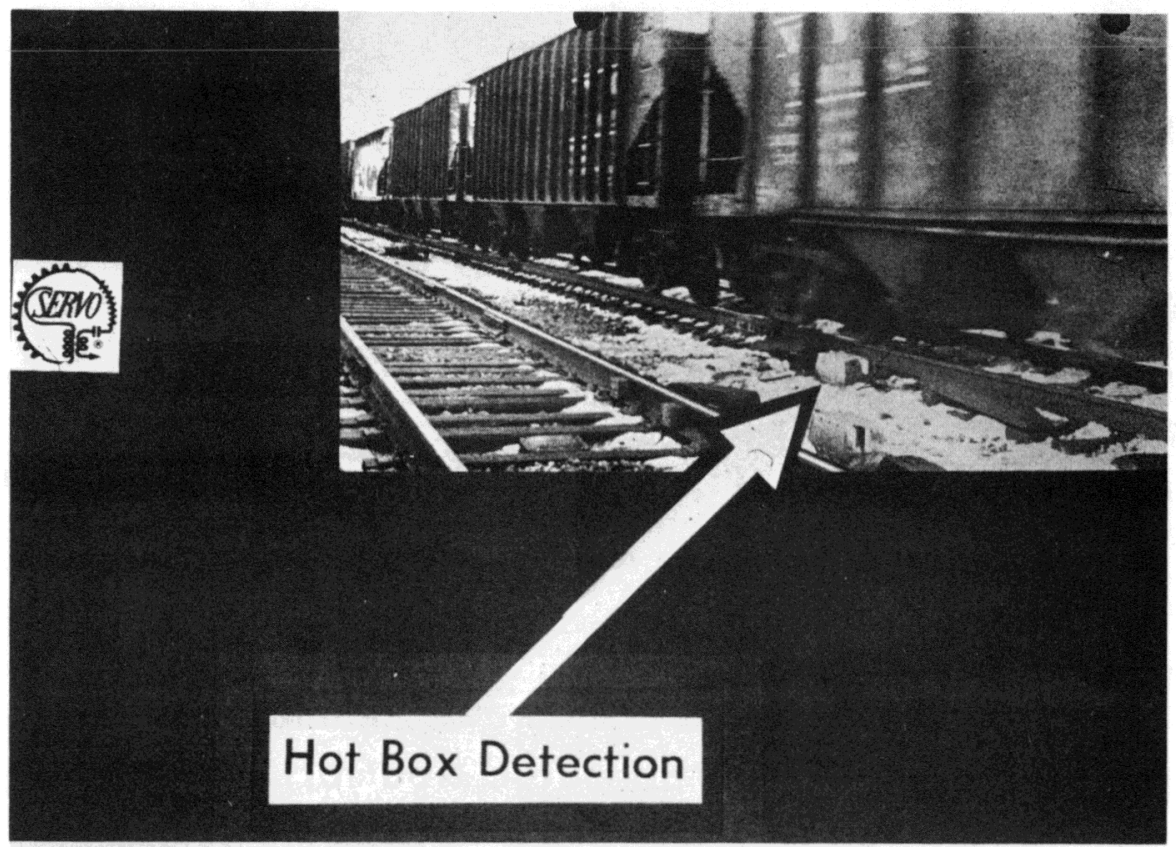

Fig. 7. Hot Box Detector

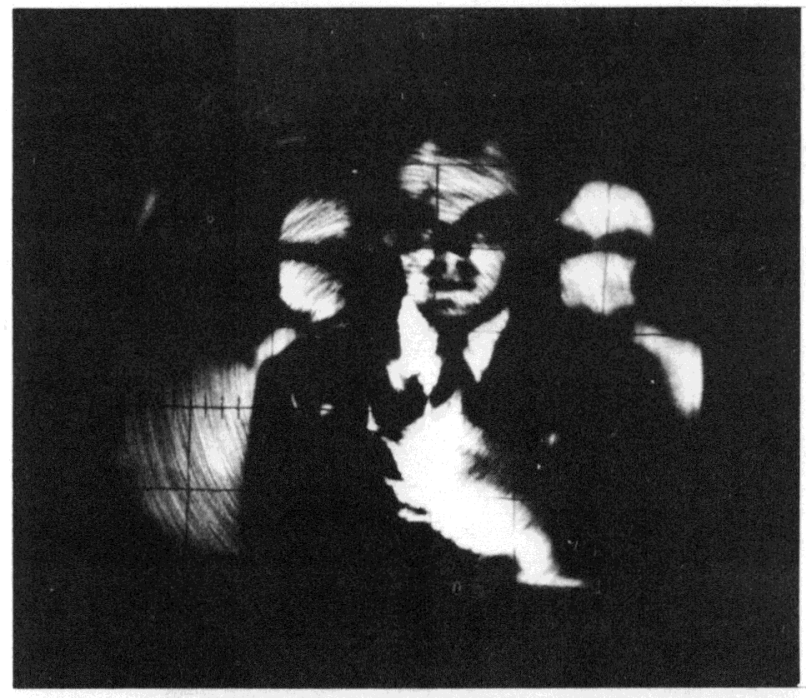

Fig. 8

Rotating Wedge Scanner Display

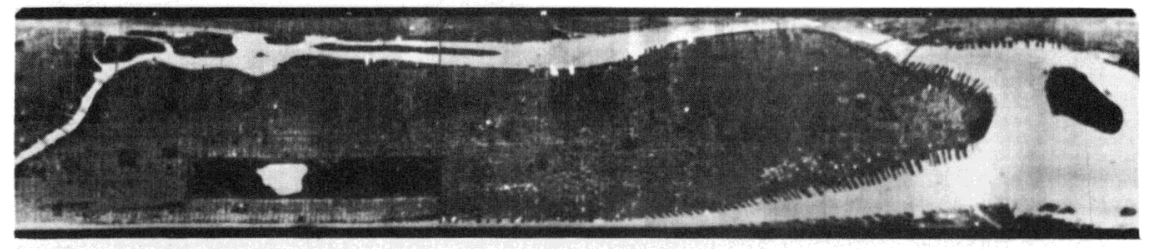

Fig. 9. Manhattan IR Map 


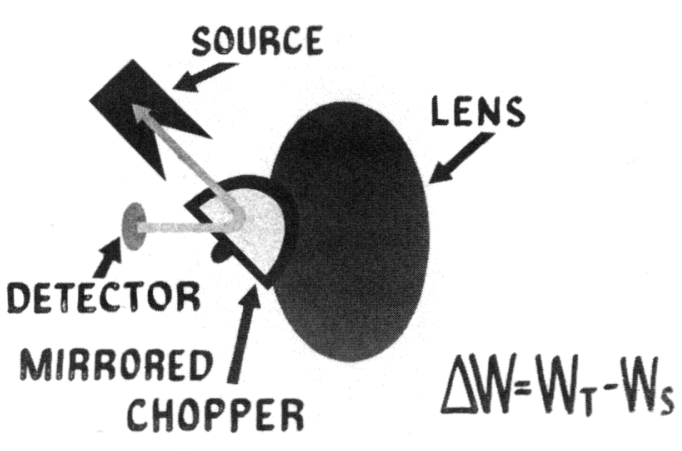

Fig. 10. Basic Radiation Measurement System
Figure 8 is an infrared display produced by a Perkin-Elmer developmental scanner. In this device the target is stationary, and rotating optical elements at the aperture perform a "flying spot" spiral scan of the object. The recorded signals are produced by thermal emission in the $4-$ to $5-\mu$ region.

An infrared reconnaissance map of Manhattan Island, Fig. 9, indicates the capability of a scanning system developed by Haller, Raymond and Brown.

The device is classified as a line scanner wherein a rotating mirror performs a flying spot scan along a line perpendicular to the flight path. Proper registration of this sequence of scans requires a precise setting of the display relative to the aircraft ground velocity.

The group of devices described above are basic radiation-detection systems with varying degrees of sophistication, the output signal being derived directly from spatial changes in object emission.

Another important class of instrumentation specifically designed for measurement of emission level is illustrated in Figure 10. Key elements in this instrument include a mirror chopper and internal reference source interposed in the radiation path to the detector. The object emission is sequentially compared with the radiation level of the internal reference source, through path switching of the rotating chopper mirror. This technique permits accurate measurement of the quiescent emission from stationary surfaces, relative to a standard. In many measurement situations, the surface under study is at an elevated temperature and correspondingly high quiescent emission level. Thermal gradient details of interest, however, represent point-to-point variations of only a few degrees centigrade. The internal reference source can be employed to offset this quiescent emission by adjustment of its temperature, and the low-level fluctuations can be studied in minute detail.

A pictorial of the radiometer employed in our rocket engine flaw study is shown in Fig. 11. The device makes use of all-reflecting optics for thermal emission measurements in the 7.5-14 $\mu$ region. A field stop is employed at the focal plane of the collector system, thereby insuring precise determination of the viewing field of the radiometer. Energy passing through the field stop is collected by a "folded" ellipsoid condenser system and focussed on the detector at 4:1 demagnification. A thermistor detector with a time constant of one millisecond is employed for these measurements. Setup and focussing of the instrument are simplified by the reflex visual viewing system, eliminating parallax problems at the short object distances of 36 in. 


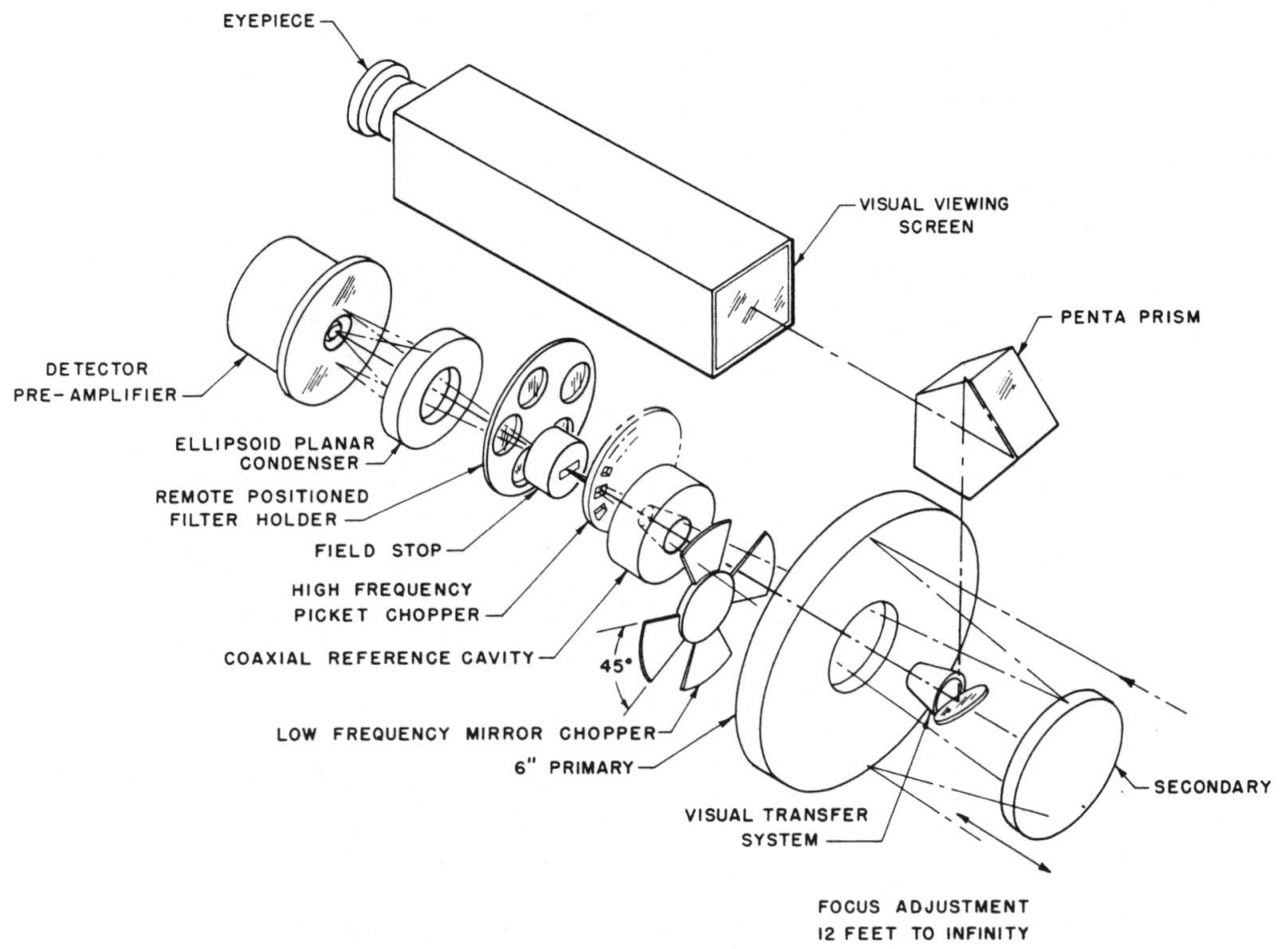

Fig. 11. Peco Research Radiometer

III. Thermal Infrared Inspection Technique (TIRI)

The infrared emission from the surface of solid propellant missile casings heated under precisely controlled conditions shows gradient effects highly correlated with underlying bond defects at the casing-liner and liner-propellant interfaces. In this section we briefly review some of the results of our initial studies. The developmental technique embodies a number of key operational principles, noted below:

(1) Heat energy is injected and emission measurements made on the single, exposed specimen surface.

(2) Heat energy is injected into the test specimen over a small area surrounding the point of subsequent measurement on a continuous scan mode principle. 
(3) Variations in volume heat diffusivity in the vicinity of flaw regions give rise to a time-varying temperature gradient characteristic, sensed as an infrared emission from the surface.

(4) Evaluation of the fluctuations of infrared emission is achieved by comparison of the observed signal to a "normal" signal arising from a flawless region.

(5) It is postulated (and demonstrated) that the departure of the surface emission characteristic from "norm" in the vicinity of internal flaws is a dynamic effect, with contrast significantly affected by what we call the exposure time. This factor is the delay or lag between the instant of injection of heat energy and the moment when thermal emission from the exposed area is observed. Optimum exposure time varies with the structure and thermal properties of the material under test and the desired depth of penetration.

The initial stages of the program were concerned with detailed analysis of heat-flow mechanisms in typical laminate structures and at interface discontinuities. The analysis made recourse to formal solutions of the partial differential equations governing these heat-flow mechanisms, and were later extended to passive electrical analogue simulation. Figure 12 is a passive circuit analogue of the heat-diffusion process surrounding a point on the specimen surface. This analogue approximates radial (surface) and transverse (inward) heat diffusion in a typical structure. Input current is analogous to injected heat energy, and the time-varying voltage at nodes along the line is directly proportional to instantaneous temperature at varying depth. Switch " $\mathrm{S}_{1}$ " simulates a void defect between steel casing and insulating liner, and " $\mathrm{S}_{2}$ " a void between liner and propellant grain. The voltage analogue of interest is observed at the point of current injection associated with the casing surface.

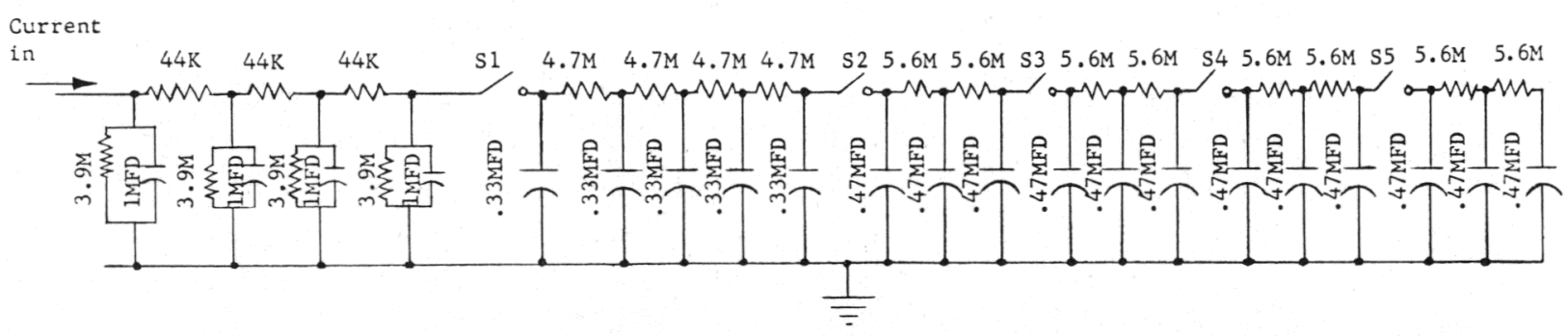

Fig. 12. Heat Diffusion Electrical Analogue

Because the passive analogue employs fixed resistive and capacitive elements, the radial diffusion process can only be roughly approximated. The 3.9-megohm reciprocal conductance elements shunting the simulated "steel-casing" network are compromise values that empirical results later showed to be low by a factor of about 3:1. Figure 13 illustrates the radial diffusion process observed from a sequence of infrared-emission scans of a casing sample. This initial heat injection area is about $1 \mathrm{~cm}^{2}$. From 
the curves, the ratio of peak relative amplitude between the first and second scans taken $7.0 \mathrm{sec}$ apart is equivalent to a time constant of about $8 \mathrm{sec}$.

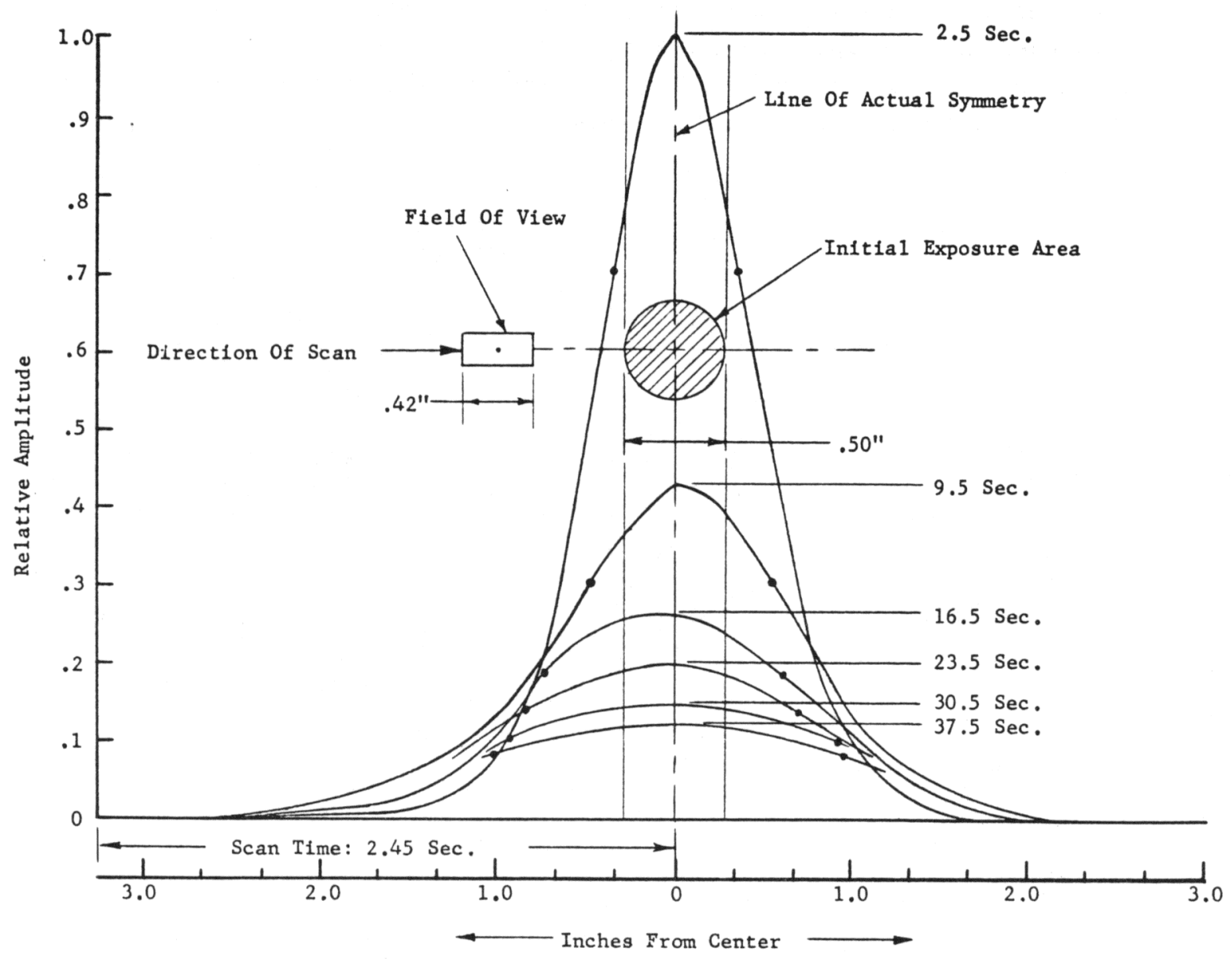

Fig. 13. Lateral Diffusion Measurements

One of the key problems involved with the early development related to the specific technique for heat injection and to the high power levels required (200-1000 watts). Following an exploratory study of magnetic induction heater apparatus, the experimental system incorporating a $2.0-\mathrm{kw}$ unit was put into operation early in 1961. The incorporation and "prove-out" of the magnetic induction heater is a significant milestone in the progress of this program. Evidence of the capability and practicability of this technique for efficient, uniform, and stable injection of heat energy at levels compatible with the measurement requirements, marked the final solution to this key problem. We immediately could conceive the practicable realization of continuous scan inspection with area coverage rates in excess of $1 \mathrm{ft}^{2} / \mathrm{min}$. 


\section{A. Experimental Scanning System}

Figure 14 is a full-scale outline of the 6 -in.-square test plate. The broken line, inset $0.30 \mathrm{in}$. from the heavy boundary, indicates the edge of the liner-propellant backing, which has been cut away to permit mounting of the specimen in a test fixture for these studies. The steel plate is typically $0.125 \mathrm{in}$. in thickness.

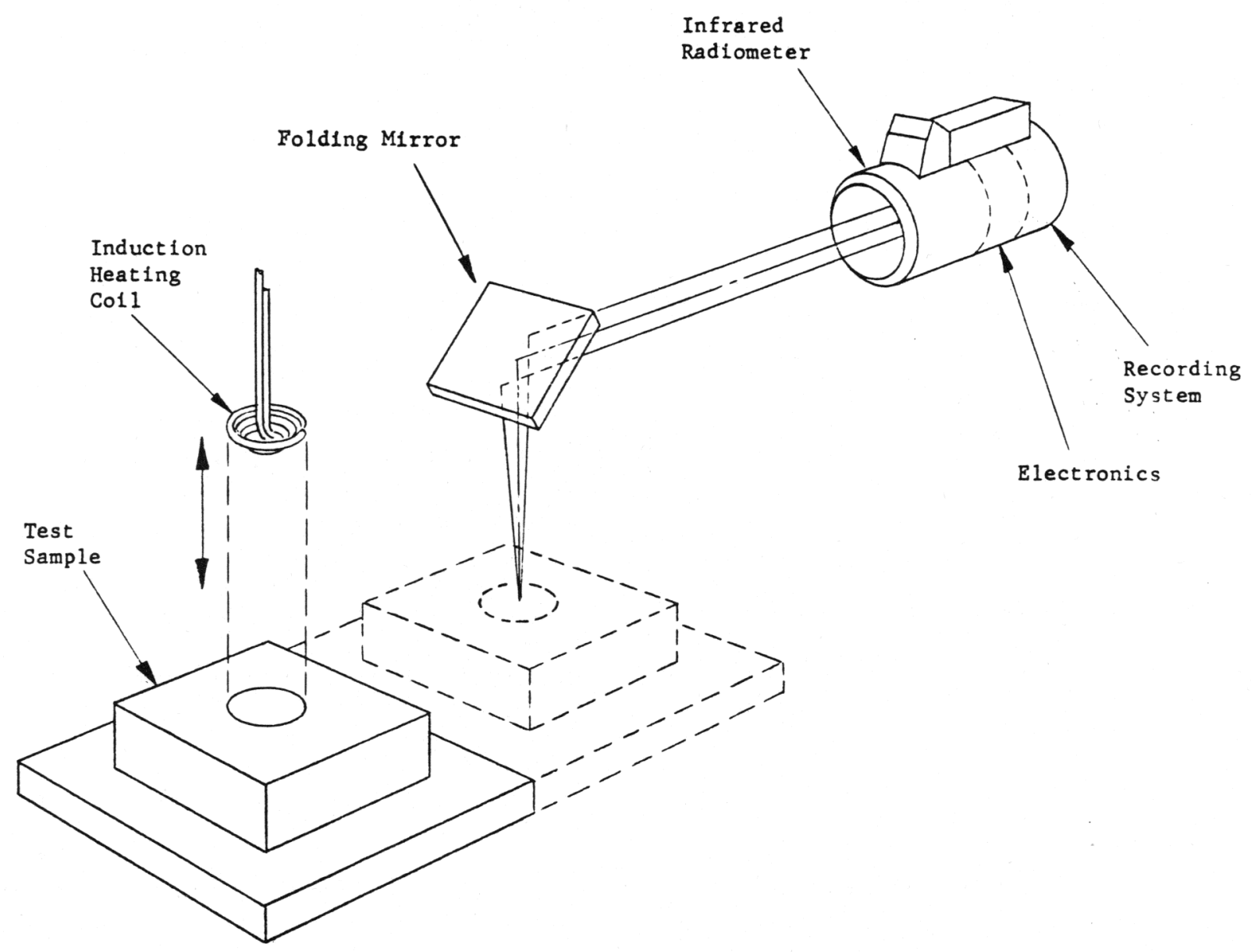

Fig. 14. Experimental "TIRI" System

The groups of arrows arranged about the edges of the figure depict the direction of sequential line scans created by the apparent moving heat source (induction heater coil) and subsequent radiation measurement. At the end of each scan, the plate is returned to its initial position and indexed 0.25 in. for the next line scan along the adjacent strip.

Implementation of this scanning procedure is achieved with the experimental setup illustrated in Fig. 15. 
1) Direction of arrows denotes direction of sequential scan strips for each of 4 emissographs.

2) Each line scan extends from corresponding edge to center of specimen (3.0"). Adjacent 11nes .25" between centers.

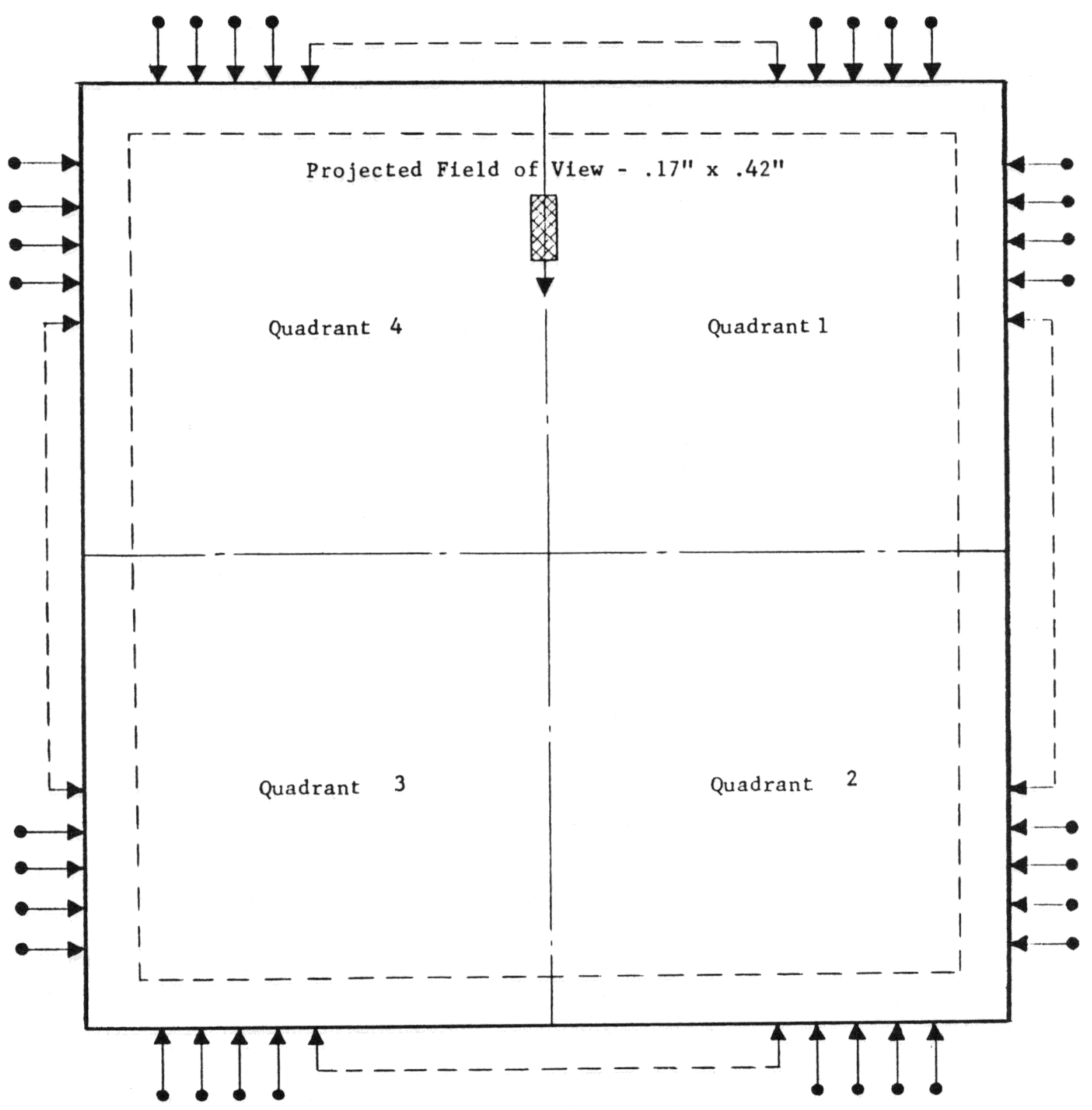

Fig. 15. Specimen Scan Mode

Linear motion of the test specimen relative to the stationary induction heater coil and radiometer field-of-view employs a hydraulicpneumatic slide assembly. A range of linear motion rate to $3 \mathrm{in} . / \mathrm{sec}$ is available with this device. 
The sequential scan emission data are applied to the recorder ordinate axis, while the abscissa axis recorder deflection is synchronized with the specimen table motion. The resultant Emissograph embodies all the information needed for detection and interpretation of flaw regions.

B. Measurement and Interpretive Procedures

The point-to-point specimen thermal emission arising with continuous line scan operation is recorded as the detected radiometer output signal. A composite set of parallel, overlapping line scans over a section of test specimen area constitutes an Emissograph display. An X-Y recorder is employed as the display medium. The emissograph embodies all the information needed to detect and evaluate possible flaw regions.

Figure 16 is an emissograph of a thermally isolated, accurately machined reference plate, 1020 steel alloy, 0.125 in. in thickness, taken under standard dynamic conditions. The flatness of the plateau regions is strikingly notable, indicating:

(1) the isothermal nature of the received signal;

(2) the complete absence of variable emissivity effects after surface treatment with a blackening agent; and

(3) the stability of the induction heater energy injection.

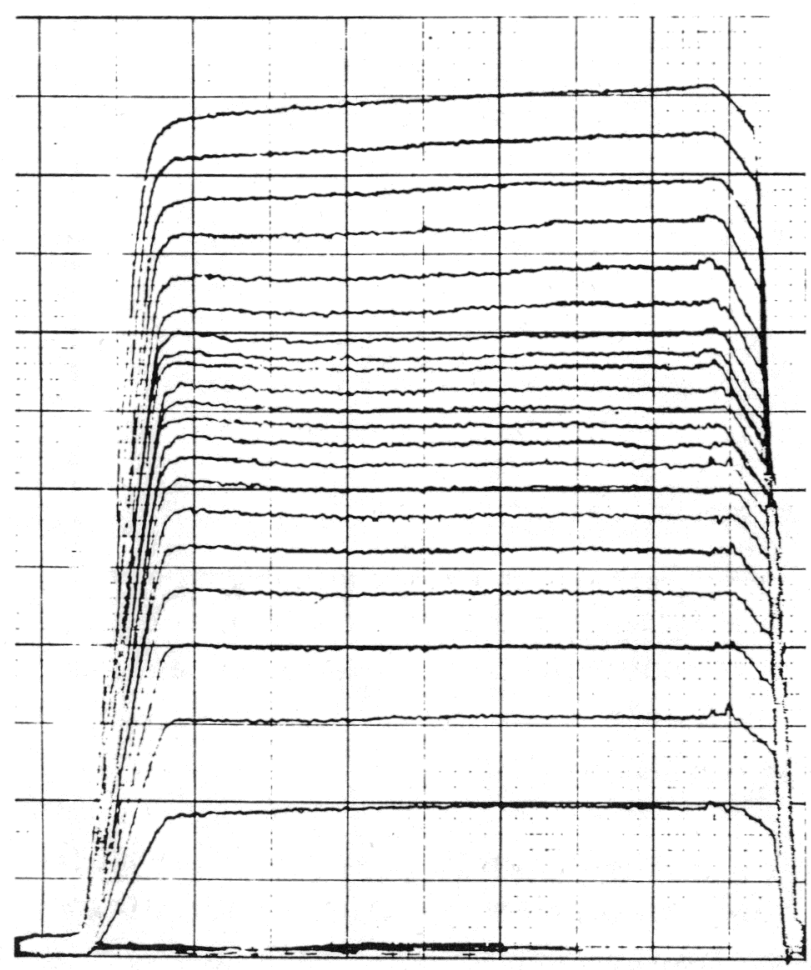

Starting Coordinates: Q4 - 1/2" Off Right Edge Scan Direction: Q4 Right to Q1 Left in 1/4" Steps Recorder Speed: 2 Seconds Per Inch Table Speed: 2.5 Seconds Per Inch Calibration: 150 Microvolts Per Inch Temperature Calibration: 5.5 Volts $/{ }^{\circ} \mathrm{C}$ (nominal)

Fig. 16. Reference Plate Emissograph 
Figure 17 is typical of a set of emissographs resulting from examination of a test specimen. Each emissograph covers 50 percent of the sample area. The ordinate scale is proportional to the surface emission from a field-of-view $0.17 \times 0.42$ in. along the scan strip, and the abscissa proportional to both real time and displacement of the sample plate in the direction of continuous scan.

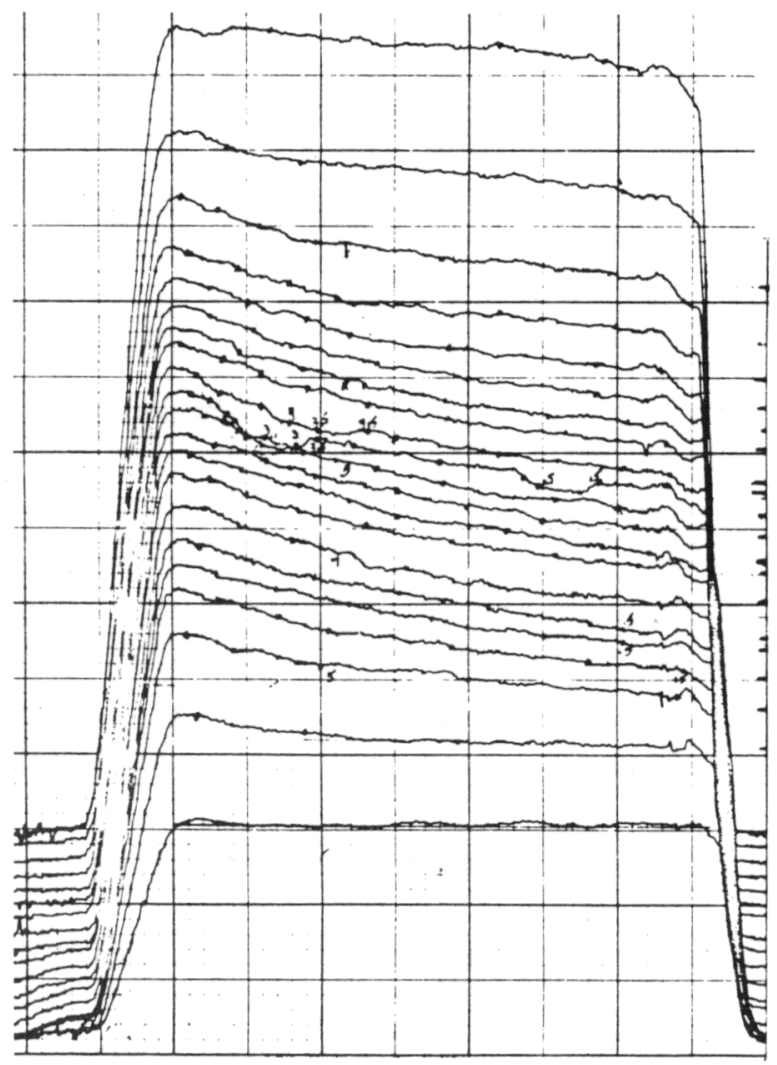

Starting Coordinates: Q1 - 1/2" Off Right Edge Scan Direction: Q1 Right to Q2 Left in 1/4" Steps Recorder Speed: 2 Seconds Per Inch Table Speed: 2.5 Seconds Per Inch Calibration: 100 Microvolts Per Inch Temperature Calibration: 5.5 Volts $/{ }^{\circ} \mathrm{C}$ (nominal)

Fig. 17. Test Specimen Emissograph

For each of the traces, the steeply rising left-hand slope is the received emission as the edge of the sample plate passes through the field of view. The "plateau" regions indicate emission fluctuations arising from variations of surface temperature along the scan line, whereas the terminating decay of the signal level results from rapid return of the plate to its initial position, immediately after completion of the scan. The offset between line traces arises through the steady temperature rise of the plate with the continuous heat injection, and, after thermal steady state, manipulation of the recorder "zero" by the technician.

The degree of departure of the "plateau" regions from true flatness is an accurate measure of the temperature variations arising from nonuniformities and bond flaws within the specimen. 
The emissographs are used to create a set of contour plots. Each scan line is analyzed by establishing a minimum level for each line, points on the scan line extending above this minimum being graded on a voltage scale with increasing voltage the further from the minimum level. The values used are 5, 10, 20, 30 microvolts above minimum level. These points are then transferred to appropriate locations on a diagram representing the surface of the sample and corresponding points are connected. The contours are not true isotherms; the signal data have not been converted to absolute temperature for the presentation. They are, however, contours of fixed radiation signal departure from flatness, and, as such, are an approximate, but reasonable, indication of dynamic thermal patterns.

The contour map, Fig. 18, and orthogonal map, Fig. 19, serve primarily as an aid to visualization and interpretation, and do not per se provide more information than is available on the emissographs. Advanced display techniques are presently being developed to eliminate the intermediate step of manual contour plotting.

The flaw pattern, Fig. 20, is then established by evaluating the resultant contour plots. Regions labeled flaw (i) are typical of areas in which there is strong evidence of defects when scanned in both directions. Regions labeled flaw (ii) and flaw (iii) were those in which weaker flaw indications are obtained for scans in both directions.

It is significant to note that delayed emissographs of test samples taken 15 to 20 sec after heat injection present plateau uniformities essentially the same as those typified by the reference plate. As predicted, the appearance of temperature variations is a true dynamic effect, with the signal contrast determined in large degree by a factor analogous to exposure time in the photographic arts, i.e., the time delay between the injection of heat energy and the observed emission from the area exposed. For the construction and steel thickness characterizing the specimen, a 6 -sec delay is about optimum for maximum contrast in first interface bonding flaws.

Of even greater interest, however, are the subtle changes that we have observed in the emissograph characterisitic, with delays (or exposure times) of the order of $30 \mathrm{sec}$, the nominal optimum exposure for liner penetration. Evidence for second interface flaw detectability, based on recent measurements, is highly promising. 


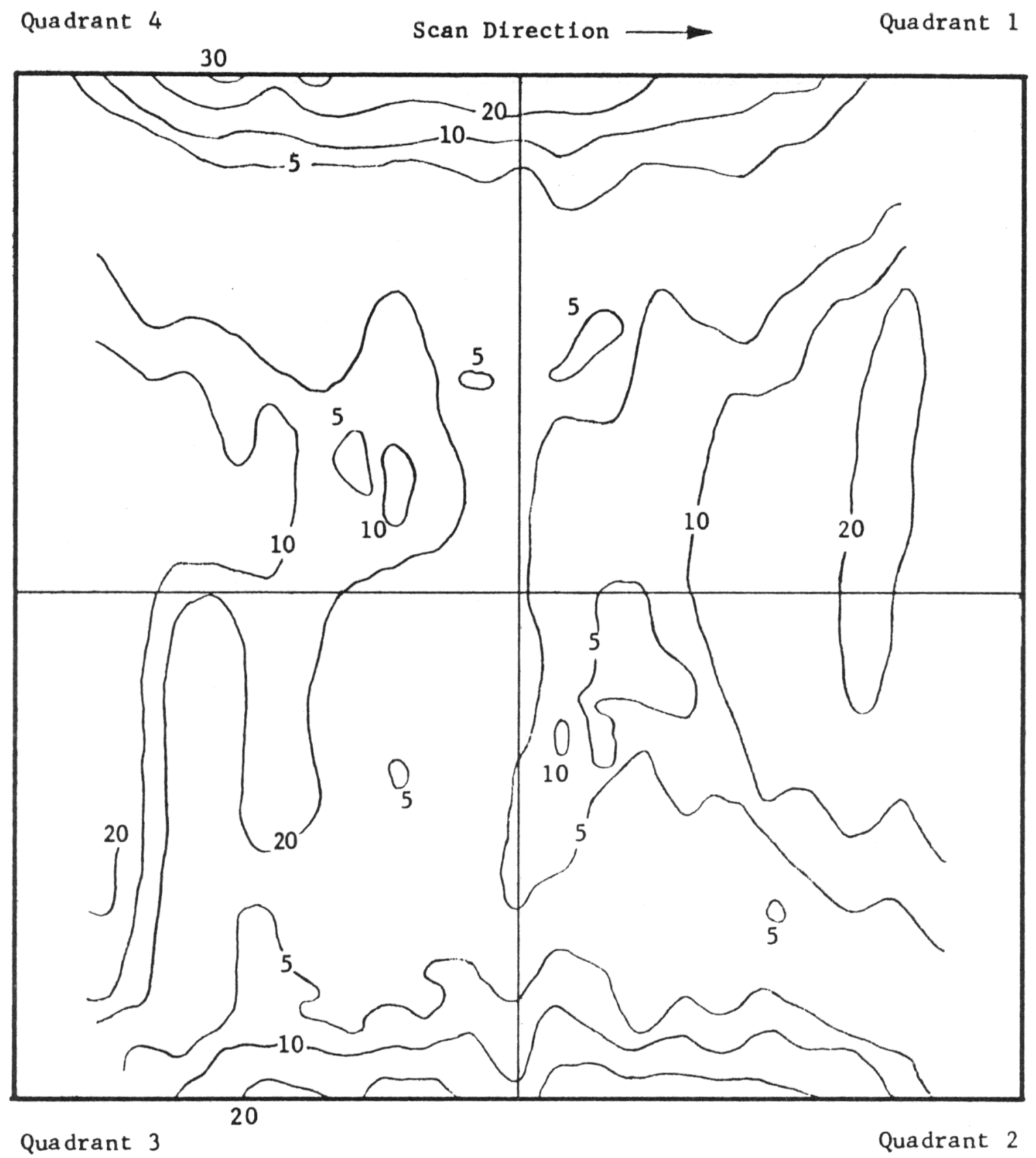

Fig. 18. Test Specimen Contour Plot 
Quadrant 4

Quadrant 1

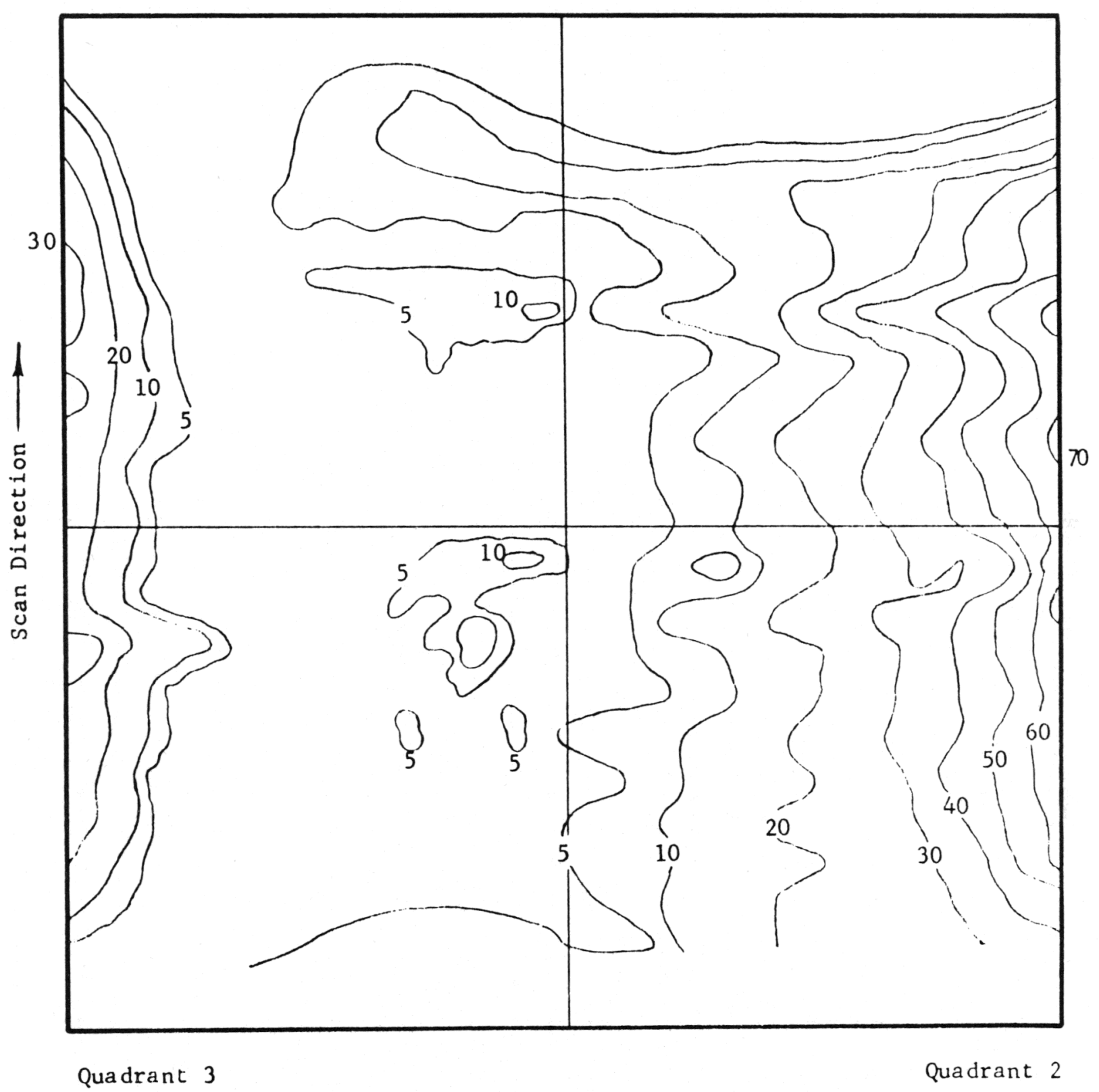

Fig. 19. Orthogonal Contour Plot 


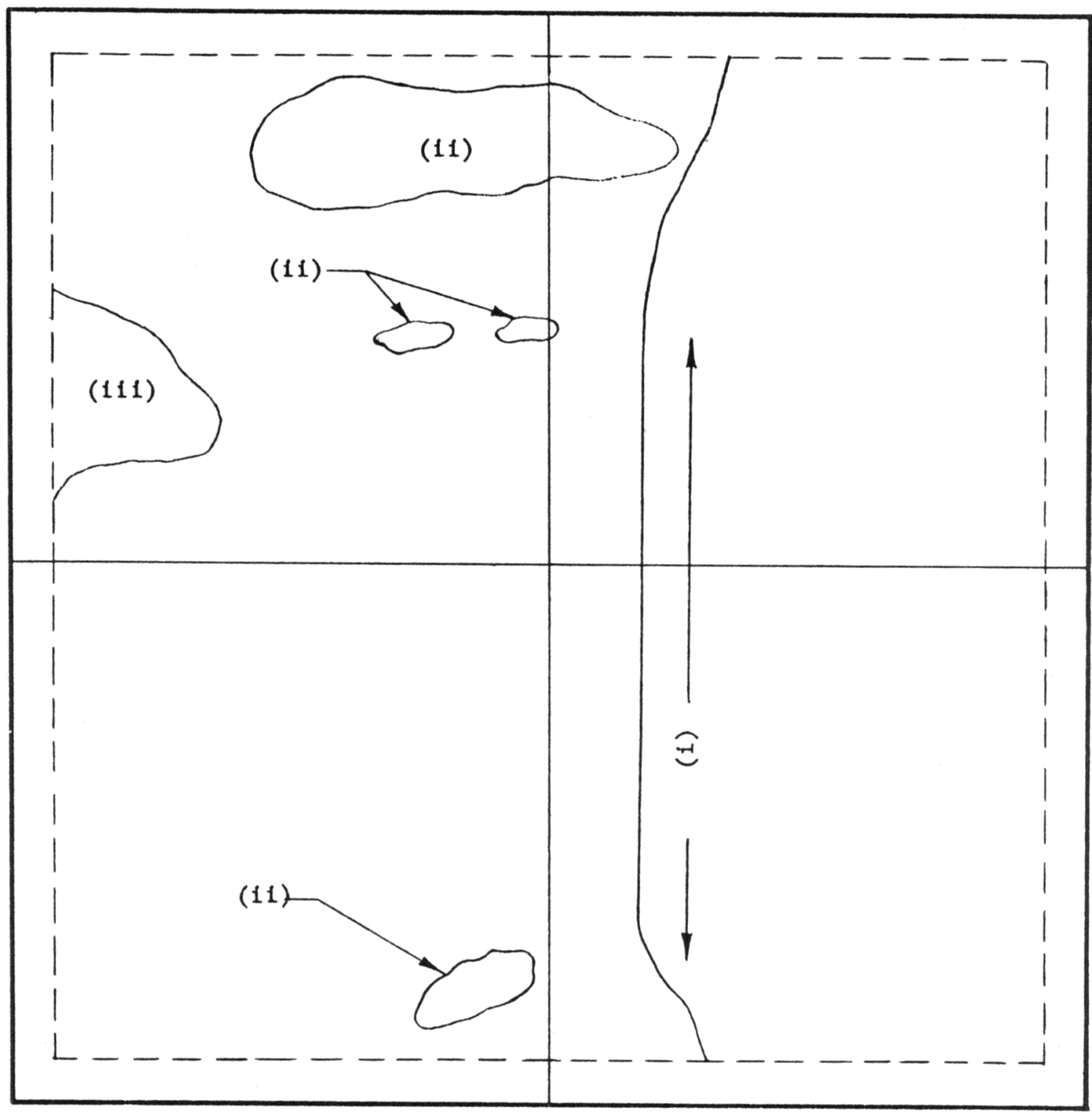

Quadrant 3

Quadrant 2

Fig. 20. Suspect Flaw Regions

\section{Summary}

The Thermal Infrared Inspection "TIRI" technique described herein has demonstrated the capability to define first interface voidtype defects 0.250 in. $^{2}$ in area or larger with 90 percent probability for detection. More subtle flaws synthesized as a cellophane adhesive 
tape interposed between casing and liner and with no observable void have been detected for areas of one in. ${ }^{2}$. Synthesized gross second interface voids of one-in. diameter are routinely defined by means of this technique.

The system employs conventional induction heater apparatus and a concentrator coil assembly for continuous injection of heat energy into elemental adjoining areas of the specimen, with subsequent radiometer measurement of the emission from the heated regions. An intrinsic property of the technique permits segregation of the defects by interface location or depth, through application of the exposure time principle.

The rate of area inspection is limited only by the output power available from the rf induction generator; one square foot/minute being readily achieved at an injection level of about 1000 watts.

\section{Acknowledgement}

Section III of this presentation briefly reviews initial results of an experimental test program leading to the development of a practical Thermal Infrared Nondestructive Inspection Technique for application to solid propellant rocket engines. The work is being supported and monitored by the U.S. Army Rocket and Guided Missile Agency, Redstone Arsenal and the Ordnance Materials Research Office, Watertown Arsenal, under Contract No. DA-19-020-ORD-5243.

Figures in this paper are reproduced with the permission of the U.S.Army Rocket and Guided Missile Agency. A complete reporting of these studies is made in Perkin-Elmer Report No.TR-7022, Feasibility Study of a Non-destructive Testing Infrared Inspection System for Bonding Flaw Detection, December 31, 1961. The author acknowledges the major contributions of $\mathrm{R}$. Gorman and P. Yettito of Perkin-Elmer Corporation to the progress of this program.

\section{Discussion}

\section{INFR ARED PHYSICS}

W. L. Donaldson, Southwest Research Institute: The equations for your thermal flux are identical, except for constants, with your magnetic and electrostatic equations. A question comes to my mind. What resolution can you get in terms of defect size, and at what level do you expect this to be covered up by the emissivity variations at the surface?

H。 L. Sachs: We're looking for contrast ratios of the order of 0.25 to 4 percent. In other words, as sume that the plate, or area of a specimen, would normally reach a temperature $\mathrm{T}$ with a given injection 
of energy; because of an internal flow in that vicinity there's a difference in temperature, $\Delta \mathrm{T}$. This ratio $\Delta \mathrm{T} / \mathrm{T}$, which is invariant except for the thickness of the material and other certain physical characteristics, is about 0.25 to 4 percent.

P. E. Dempsey, U.S. Steel: You measured the effects of nonadhesion of the bond. Would you comment on the method of ultrasonic testing that adhesion.

H. L. Sachs: I believe it was a very conventional pulse-echo technique. One company could not couple to the plate and had to use waterbath emersion, while another company was able to oil couple.

\section{P. E. Dempsey: Are these defects in the bonding?}

H. L. Sachs: Yes. In some cases, there is a separation that exists, and, in other cases, there is a surface contact but a lack of bond.

J. J. Boulanger, Texas Instruments, Inc.: How high a temperature did you reach on the outer plate surface with your induction heater?

H. L. Sachs: The induction heater we're using is capable of in-

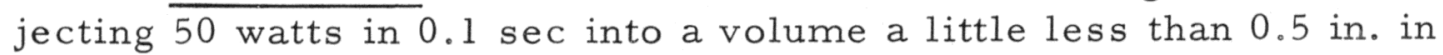
diameter by about $0.125 \mathrm{in}$. thick. The heating capabilities are so great that we are able to boil water instantaneously. Conventionally, however, we operate about $30^{\circ} \mathrm{C}$ above ambient.

J. J. Boulanger: What sort of resolution would you get if there was an unbonded area directly below this field skin? About what diameter do you feel you could see?

H. L. Sachs: We can find a 0.5-in. nonbond and a 0.25-in. void.

D. R. Green, General Electric, Hanford Atomic Products Operation: We have recently developed heat transfer tests of this type for atomic fuel elements. They are aluminum-jacketed, uranium-core fuel elements. We're using photovoltaic and photoelectromagnetic detectors of indium antimonide, and I was wondering if you could comment on the durability of the cooled indium antimonide detectors. How many thermal shocks can it be subjected to without its collapsing?

H. L. Sachs: We found that we can get an adequate number of operating hours out of the units. The problems that are usually associated with them are primarily logistic ones. Antimonide in the photovoltaic mode exhibits the kind of output that you get from a thermocouple; the noise is very low and the signal is very low. Consequently, you have to use various types of transformer coupling, and then you have a possibility 
of higher figures of system noise than if you had used other types of lead salt detectors. In general, however, with a properly designed system I think you can expect hundreds of hours, or a thousand hours of operation. I cannot say specifically how many thermal shocks it can receive.

D.R. Green: What techniques are there for preserving these detectors? It is not practical to keep them full overnight with liquid nitrogen since the nitrogen evaporates in about $15 \mathrm{~min}$, and it is not very economical to keep it full all day when it is going to be used a few times.

H. L. Sachs: You may just be filling the Dewar, and this can be inconvenient. There are available very convenient transfer systems which transfer liquid nitrogen through flexible Teflon tubing directly to the detector on a drop by drop basis.

D.R. Green: You have a flat plate which you undoubtedly scan in a video pattern type of scan. Your heat will have a chance to diffuse out from the source as you scan across the plate. Do you have any difficulties with interaction between adjacent heating scans due to the diffusion from the previous scan?

H. L. Sachs: No. On each scan we inject new energy and we tre only attempting to observe the dynamic effect of the delta-function response to the surface. The old energy that's diffused merely presents a background. It would be a problem if the emissivity were nonuniform.

L. Goodman, Atomics International: Apparently you are not cooling the plate so that you are getting a steady increase in the temperature. I take it, then, that you're having a rather limited heat flow in a direction perpendicular to the surface. Do you have anything that could be considered heat sink behind the quasi-rubber insulator?

H. L. Sachs: There is no heat sink per se, but there's a tremendous mass of liner and propellant substratum below the casing itself.

L. Goodman: Do you find that you have been detecting lack of bond in the second or third layer down?

H. L. Sachs: It looks like we have achieved successful detection of the interface, but I don't want to go on the record with a formal announcement of that as yet.

L. Goodman: Are you inclined to believe that you get your best results on a nonbond at the first interface directly below the steel plate?

H. L. Sachs: Our experimentation, thus far, has been directed primarily to the first interface. 
H. D. Hendricks, DuPont, Savannah River Laboratory: Exactly what is the separation of the nonbond region that you are testing?

H. L. Sachs: A nonbonded region can have very intimate contact under normal pressures. In other words, if there are irregularities in the surfaces, they will touch and mate, and there will be small voids in those areas which are low spots. This is as opposed to an actual void in the material where the liner or propellor has sagged away, and you have a measurable gap of 0.010 or 0.050 in.

H. D. Hendricks: What is your lower limit on the resolution of a nonbond gap?

H. L. Sachs: With regards to first interface, we've been able to resolve lack of bond in a $0.5-$ in. $^{2}$ area. It's important to point out that this is with 0.125 -in. steel. You would look for better detectability with thinner steel or thinner casings andipoorer detectability with thicker steel or casings.

W. L. Donaldson: When you use the term dynamic testing, are you talking about a transient effect in which you establish a temperature differential and in which you measure the change of temperature $\mathrm{T}_{1}$ and $\mathrm{T}_{2}$ on the interface, or do you mean establishing a steady-state condition of $\mathrm{T}_{1}$ on the interface and of $\mathrm{T}_{2}$ on the outer facing and maintaining that?

H. L. Sachs: All our observations are made during the dynamic, transient situation.

S. Leonard, Lockheed Aircraft Corporation: From the data you've presented here, I can see that you are looking at a void or lack of bond. What about the condition of a surplus of resin?

H. L. Sachs: Actually, we have not been faced with that particular kind of defect in the specimens that we have tested, so I have no observation to make.

S. Leonard: Has your work only involved the steel-jacketed type of case? Have you, in any way, looked into filament-wound cases with resin binders?

H. L. Sachs: All our specimens are steel or metal jacketed.

S. Serabian, Avco Corporation: You said that this was an 0.125-in. plate. Would you care to comment, say, on the volumetric considerations of such a technique? Suppose you had a void in the center of a plate of a certain thickness. What do you think your capabilities would be? 
H. L. Sachs: It correlates very, very well with the thickness of the material. I think the important thing is that the area of heat injection be large in comparison to the thickness. The area of heat injection pretty well sets your resolution, and this should be larger by a factor of 3 than the thickness of the material you wish to penetrate. Now, if you observe that empirical relationship you will find that you can develop a proportional contrast condition as the thickness of the material increases, but you lose resolution when you do it.

K. Lucas, Allegany Ballistics Laboratory: A good deal of work is being done with fiberglass cases. Have you been doing any work with fiberglass?

H. L. Sachs: No, our investigations have been restricted to steel casings.

K. Lucas: What type of results do you think you would be getting with fiberglass with approximately the same thickness as the steel?

H. L. Sachs: Well, one of the key components in the system is the means for injecting the energy. We played around with other approaches, and we found that the most satisfactory by far was induction heating. 


\title{
THER MISTORS
}

by

Robert S. Goodyear

Fenwal Electronics, Inc.

Framingham, Massachusetts

\begin{abstract}
This discussion will include a brief survey of the various types and sizes of thermistors currently available with information about their electrical and thermal characteris tics, including resistance-temperature, voltage-current, and current-time characteristics, as well as stability, reproducibility, precision, and range of reliable operation. Applications will be mentioned to show how the different characteristics are used.
\end{abstract}

Thermistors were named by people at the Bell Telephone Laboratories as a contraction for thermal resistors. They are resistors with a high temperature coefficient of resistance and their resistance is a function of absolute temperature. Thermistors are not new. They have been known for about 150 years but have had very little use because they were considered to be unstable and nonreproducible. This is no longer true.

About 40 years ago, the Bell Telephone Laboratories started investigating various materials and processing procedures to determine how to make stable and reproducible thermistors. They were quite successful in their investigations and actually put thermistors into large-scale telephone usage about 21 years ago. The units they developed were both highly stable and closely reproducible to standard specifications. It is only in the last 10 years, however, that thermistors have been generally available for large-scale industrial use.

Many interesting applications for commercial and industrial use of thermistors have been developed in the last few years, although such development has been very slow because of the lack of trained engineers who know how to use thermistors in electrical and electronic circuits. Thermistors are now available in a large variety of types covering a broad range of electrical characteristics. There are discs, washers, rods, beads, wafers, and flakes capable of handling from a few microwatts to 25 watts of power and having a resistance range from a fraction of an ohm to $100 \mathrm{meg}-$ ohms with time constants from a fraction of a millisecond to several hours. Resistance values are available in normal tolerances of 10 to $20 \%$, but many types can be obtained with tolerances as low as $0.1 \%$. With this vast range 
of values available to engineers, it is no wonder that thermistors are finding their way into new equipment daily.

Thermistors are made of oxides of such metals as manganese, nickel, cobalt, and copper. The oxides are formed into various shapes and sintered at high temperature between 1100 and $1400^{\circ} \mathrm{C}$. They become a hard, ceramic material, with a specific gravity of about 5, and may have leads attached to them by firing on silver or platinum paste to form a conducting surface.

The disc is the simplest shape because the powdered oxide can be pressed in a round die. Various resistance values can be obtained by varying the thickness of the discs. Standard disc diameters are 0.1, 0.2, 0.3, 0.4, $0.5,0.6,0.75$, and $1.0 \mathrm{in}$. Thicknesses range from 0.015 to 0.5 in. The resistance of discs at $77^{\circ} \mathrm{F}$ varies from a fraction of an ohm to $10,000 \mathrm{ohms}$. Most thermistors have temperature coefficients of about 2 to $3 \%$ per ${ }^{\circ} \mathrm{F}$.

Washers are simply discs with holes in the center for easy mounting on bolts and for multiple stacking when more-power handling capacity is required.

Rods are extruded to form cylinders $0.053,0.11$, and $0.173 \mathrm{in}$. in diameter and lengths from $\frac{3}{8}$ to $1 \frac{3}{4} \mathrm{in}$. Resistances range from 1000 to 100,000 ohms. Rods are like very thick discs to give high resistance values.

Beads are made by forming small ellipsoids of thermistor material on 2 fine platinum wires stretched tight and parallel about 0.010 in. apart. During sintering, the leads become embedded tightly in the beads, so that there is good electrical contact inside the thermistor. Beads may be coated with glass for protection, or they may be mounted in evacuated or gas-filled bulbs. Resistance values of $50 \mathrm{ohms}$ to over $100 \mathrm{megohms}$ can be obtained in beads ranging from 0.006 to $0.100 \mathrm{in}$. in diameter. Beads are also sealed into the tips of solid glass rods which are called glass-probe thermistors.

The materials and processes developed by the Bell Telephone Laboratories are for use primarily at low temperatures of $300^{\circ} \mathrm{C}$ or less. Glasscoated units may be used at $300^{\circ} \mathrm{C}$ with very high stability. If the resistance of a new thermistor is plotted against the logarithm of time during which it is held at an elevated temperature, with the time coordinates being something like 1 day, 1 week, 1 month, 1 year, 10 years, the curve will be a rising exponential curve approaching an asymptote of a fixed resistance as time increases. Such a curve might have a total change of resistance of 2 to $3 \%$ over the whole time scale, with $90 \%$ of the change taking place in the first week. This leaves only 0.2-0.3 percent change to take place through the years of use at elevated temperature. Units have been tested which were for use in gas-analysis equipment and which had been aged at $300^{\circ} \mathrm{C}$ for 60 days and found to be stable over a period of several months to better than $0.01 \%$. This is, of course, an ideal application in which the temperature 
of the thermistor is nearly constant during its useful life. Glass-coated, bead-type thermistors were used at $500^{\circ} \mathrm{C}$ for a period of 3 months with less than $0.1 \%$ change in resistance.

In 1956, I made some precise measurements on a group of thermistors which have since been used as reference units for temperature measurements from $-100^{\circ} \mathrm{F}$ to $400^{\circ} \mathrm{F}$. These units have been cycled over this range many, many times during the last 5 years. I selected one of these units and had some tests run on it the other day so I could give you some concrete evidence of stability. All tests were made in constant-temperature baths controlled with thermistor controllers having a sensitivity of $0.0002^{\circ} \mathrm{F}$ and actually controlling to about $0.003^{\circ} \mathrm{F}$. Temperatures were checked with a Bureau of Standards-referenced platinum thermometer. Resistances were measured with standard laboratory-type Leeds and Northrup Wheatstone bridges accurate to $0.1 \%$.

We started at $77^{\circ} \mathrm{F}$, reduced the temperature to $-76^{\circ} \mathrm{F}$, and then raised it to $77^{\circ} \mathrm{F}$, stopping at various temperatures only long enough to make measurements. We measured resistance at the same temperatures on the way up as we did on the way down to show there is absolutely no measurable hysteresis in these thermistors.

We then heated the test bath up to $300^{\circ} \mathrm{F}$ and cooled it back down to $77^{\circ} \mathrm{F}$, again checking for hysteresis. We compared these readings with those taken in 1956 and found all of them within the limit of accuracy of the Wheatstone bridge!

\begin{tabular}{|c|c|}
\hline${ }^{\circ} \mathrm{F}$ & Down \\
\hline 77 & 4,002 \\
\hline 41 & 9,132 \\
\hline 30 & 11,999 \\
\hline 0 & 26,650 \\
\hline-10 & 35,430 \\
\hline-20 & 47,600 \\
\hline-76 & 307,600 \\
\hline
\end{tabular}

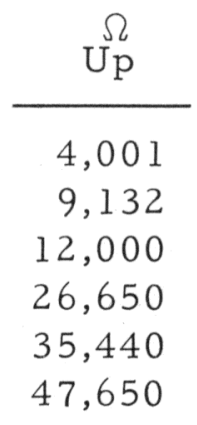

$\Omega$

Down

$\frac{{ }^{\circ} \mathrm{F}}{77}$

77

140

194

200

266

300

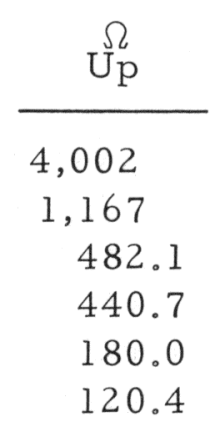

\begin{tabular}{c}
$\Omega$ Years Ago \\
\hline 4,002 \\
9,125 \\
12,000 \\
26,600 \\
35,390 \\
47,560 \\
308,000
\end{tabular}

$\Omega$

\begin{tabular}{c}
5 Years Ago \\
\hline 4,002 \\
1,166 \\
482.2 \\
440.5 \\
179.8 \\
120.3
\end{tabular}


A few tests have been made on the effects of radiation on thermistors with rather encouraging results. In some work done at Hanford, they were exposed to gamma radiation at $1.3 \times 10^{6} \mathrm{r} / \mathrm{hr}$, fission spectrum, for over $400 \mathrm{hr}$ with no damage measurable. In another series of tests, they were exposed to neutron radiation for $2172 \mathrm{hr}$ with a flux density of $4.5 \times 10^{6} \mathrm{n} / \mathrm{cm}^{2} / \mathrm{sec}$ for a total dose of $3.5 \times 10^{13} \mathrm{n} / \mathrm{cm}^{2}$ with no damage resulting.

Exposure to 2-Mev electrons for $20 \mathrm{~min}$ in a Van de Graaff generator, giving a total dose of $10^{9} \mathrm{rads}$, gave no damage.

There are 4 major groups of uses of thermistors. First are those using the basic resistance-temperature characteristics, such as temperature measurement, temperature control, and temperature compensation. Second are those using the voltage-current characteristics, such as voltage regulation, current regulation, power-level control, and volume limiting. Third are those using the time-current characteristics, such as timedelay relays, integration of power pulses, and memory in computer circuits. Fourth are those using various characteristics of thermistors in combination to measure some physical property other than temperature that can be converted into temperature by circuitry, such as gas analyzers, highfrequency power meters, flow meters, liquid level meters, and pressure or vacuum gauges.

In the first group, in which the resistance-temperature characteristic shown in Fig. 1 is used, there are a number of important considerations which make thermistors better than other devices for measuring and controlling temperature. The resistance of a thermistor is a function of

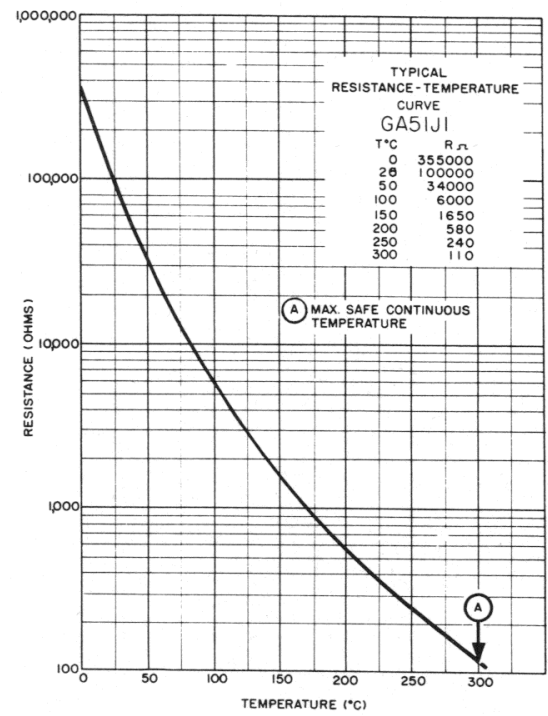

Fig. 1 its absolute temperature, and it is merely necessary to measure resistance to know the exact temperature of the thermistor, regardless of how it got there, whether by ambient temperature, self-heat, indirect heater power, or radiation. Since thermistors can have high resistance values, it is possible to measure them with moderately high potential and lowcurrent circuits, thereby eliminating the necessity of heavy conductors and sensitive measuring equipment. No reference temperature is necessary as it is with thermocouples, no special leads are required, and wiring can be with any ordinary copper wire. Sensitive, low-current instruments are not necessary and simple ohmmeters can do a fine job. If multiple circuits are desired, it is easy to switch from one thermistor to another with ordinary switches, because high voltages and low currents are used instead of low voltages and high currents as with thermocouples. By using high-resistance thermistors, remote measurements 
can be made without any special wiring, because the change in resistance of copper wires with ambient temperature is so small compared with the value of the thermistor that negligible error is introduced by such a transmission line. Vapor pressure and liquid expansion types of instruments cause much trouble for long-distance transmission, because of their extreme sensitivity to ambient temperatures around the transmission lines. Platinum wire thermometers vary only by a factor of 10 to $l$ over the range from $-100^{\circ} \mathrm{C}$ to $400^{\circ} \mathrm{C}$, whereas thermistors vary by a factor of $10^{6}$ to 1 , as shown in Fig. 2. Thermistors, therefore, when properly used, can give greater precision of measurement and control of temperature for less cost than other means.

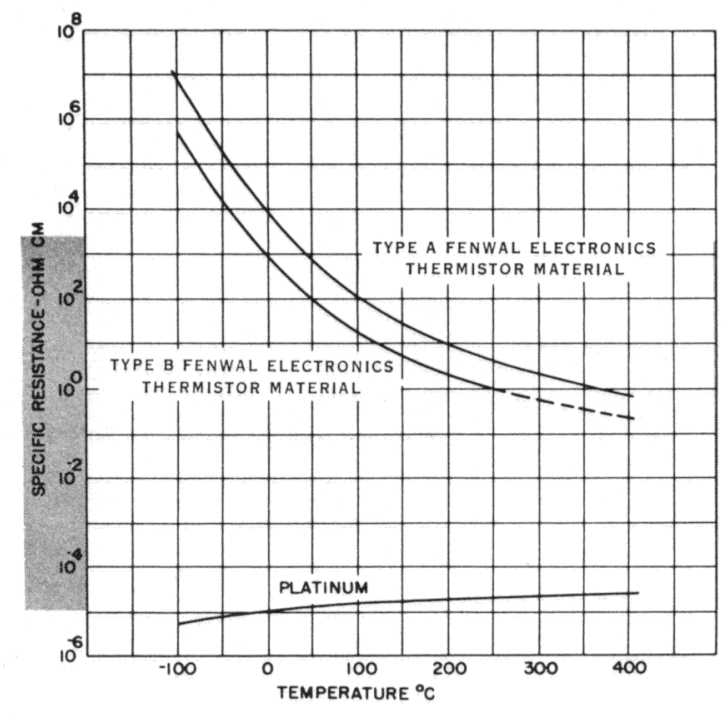

Fig. 2

One well-known use of thermistors is for measuring the engine temperature in automobiles. A thermistor disc is mounted in a probe which screws into the engine block, so that the probe is in the water jacket. The thermistor is connected in series with the battery and the indicating instrument on the dash panel. These instruments are particularly good because they are rugged and free from damage due to vibration.

Thermistors are now available which will match a predetermined resistance-temperature curve at all points of the temperature range desired to within $0.2 \%$, or approximately $0.1^{\circ} \mathrm{F}$. These are finding much use in guided missiles and satellites because the telemetered information is the same from all thermistors, thus eliminating costly data reduction previously required when thermistors were individually calibrated. Such a tabulation is illustrated in Fig. 3. 


\begin{tabular}{|c|c|c|c|c|c|c|c|c|c|c|c|c|c|}
\hline \multicolumn{2}{|c|}{ Resistance } & \multicolumn{2}{|c|}{${ }^{\circ} \mathbf{F}$ Resistance } & \multicolumn{2}{|c|}{${ }^{\circ} \mathbf{F}$ Resistance } & \multicolumn{2}{|c|}{${ }^{\circ} \mathbf{F}$ Resistance } & \multicolumn{2}{|c|}{ oF Resistance } & \multicolumn{2}{|c|}{${ }^{\circ} \mathbf{F}$ Resistance } & \multicolumn{2}{|c|}{${ }^{\circ} \mathbf{F}$ Resistance } \\
\hline 0 & 26520 & 51 & 7183 & 101 & 2433 & 151 & 962.8 & 201 & 433.9 & 251 & 217.6 & 301 & 118.9 \\
\hline 1 & 25790 & 52 & 7016 & 102 & 2385 & 152 & 946.5 & 202 & 427.5 & 252 & 214.8 & 302 & 117.6 \\
\hline 2 & 25080 & 53 & 6854 & 103 & 2338 & 153 & 930.5 & 203 & 421.2 & 253 & 212.1 & 303 & 116.3 \\
\hline 3 & 24400 & 54 & 6697 & 104 & 2292 & 154 & 914.8 & 204 & 415.0 & 254 & 209.4 & 304 & 115.0 \\
\hline 4 & 23740 & 55 & 6544 & 105 & 2247 & 155 & 899.5 & 205 & 409.0 & 255 & 206.7 & 305 & 113.7 \\
\hline 5 & 23100 & 56 & 6395 & 106 & 2203 & 156 & 884.5 & 206 & 403.1 & 256 & 204.1 & 306 & 112.4 \\
\hline 6 & 22480 & 57 & 6249 & 107 & 2160 & 157 & 869.7 & 207 & 397.3 & 257 & 201.5 & 307 & 111.1 \\
\hline 7 & 21880 & 58 & 6107 & 108 & 2118 & 158 & 855.2 & 208 & 391.6 & 258 & 199.0 & 308 & 109.9 \\
\hline 8 & 21290 & 59 & 5968 & 109 & 2077 & 159 & 841.0 & 209 & 385.9 & 259 & 196.5 & 309 & 108.7 \\
\hline 9 & 20720 & 60 & 5833 & 110 & 2037 & 160 & 827.1 & 210 & 380.3 & 260 & 19 & 310 & 107.5 \\
\hline 10 & 20170 & 61 & 5701 & 111 & 1998 & 161 & 813.5 & 211 & 374.8 & 261 & 191.6 & 311 & 106.3 \\
\hline 11 & 19630 & 62 & 5573 & 112 & 1960 & 162 & 800.1 & 212 & 369.4 & 262 & & 312 & 105.1 \\
\hline 12 & 19110 & 63 & 5448 & 113 & 1923 & 163 & 786.9 & 213 & 364.1 & 263 & 186.8 & 313 & 103.9 \\
\hline 13 & 18610 & 64 & 5327 & 114 & 1886 & 164 & 774.0 & 214 & 359.0 & 264 & 184.5 & 314 & 102.8 \\
\hline 14 & 18120 & 65 & 5209 & 115 & 1850 & 165 & 761.4 & 215 & 354.0 & 265 & 182.2 & 315 & 101.7 \\
\hline 15 & 17650 & 66 & 5094 & 116 & 1815 & 166 & 749.0 & 216 & 349.0 & 266 & 180.0 & 316 & 100.6 \\
\hline 16 & 17190 & 67 & 4982 & 117 & & 167 & 736.8 & 217 & 1 & 267 & 177.8 & 317 & 99.5 \\
\hline 17 & 16740 & 68 & 4873 & 118 & 1747 & 168 & 724.9 & 218 & 339.3 & 268 & 175.6 & 318 & 98.4 \\
\hline 18 & 16310 & 69 & 4766 & 119 & 1714 & 169 & 713.2 & 219 & & 269 & 173.4 & 319 & 97.3 \\
\hline 19 & 15890 & 70 & 4661 & 120 & 1682 & 170 & 701.8 & 220 & 329.9 & 270 & 171.3 & 320 & 96.3 \\
\hline 20 & 15480 & 71 & 4559 & 121 & 1650 & 171 & 690.6 & 221 & 325.3 & 271 & 169.2 & 321 & 95.3 \\
\hline 21 & 15080 & 72 & 4460 & 122 & 1619 & 172 & 6 & 222 & .8 & 272 & & 322 & 4.3 \\
\hline 22 & 14690 & 73 & 4364 & 123 & 1589 & 173 & 668.8 & 223 & 316.4 & 273 & 165.2 & 323 & 93.3 \\
\hline 23 & 14320 & 74 & 4270 & 124 & 1560 & 174 & 658.1 & 224 & 312.0 & 274 & 163.2 & 324 & 92.3 \\
\hline 24 & $\begin{array}{l}13960 \\
13610\end{array}$ & 75 & 4178 & 125 & 1531 & 175 & 647.6 & 225 & 307.7 & 275 & 161.2 & 325 & 91.3 \\
\hline 25 & 13610 & 76 & 4089 & 126 & 1503 & 176 & 63 & 226 & 303.5 & 276 & 159.3 & 326 & 90.3 \\
\hline 26 & $\begin{array}{l}13270 \\
12940\end{array}$ & 77 & 4002 & 127 & 1475 & 177 & 627.2 & 227 & & 277 & & 327 & 9.3 \\
\hline 27 & 12940 & 78 & 3917 & 128 & 1448 & 178 & 617.3 & 228 & 295.2 & 278 & 155.5 & 328 & 88.4 \\
\hline 28 & 12620 & 79 & 3834 & 129 & 1422 & 179 & 60 & 229 & .2 & 279 & 6 & 329 & 37.5 \\
\hline $\begin{array}{l}29 \\
30\end{array}$ & $\begin{array}{l}12300 \\
11990\end{array}$ & 80 & 3752 & 130 & 1396 & 180 & 598.3 & 230 & 287.3 & 280 & 151.8 & 330 & 86.6 \\
\hline 31 & 11690 & 81 & 3672 & 131 & 1371 & 181 & 589.0 & 231 & 4 & 281 & 150.0 & 331 & 85.7 \\
\hline 32 & 11400 & 82 & & 132 & 1346 & 182 & 579.9 & 232 & $2 / 9.6$ & 282 & 148.2 & 332 & 34.8 \\
\hline 33 & 11120 & 83 & 3519 & 133 & 1322 & 183 & 570.9 & 233 & 275.8 & 283 & 146.5 & 333 & 83.9 \\
\hline 34 & 10850 & 84 & 3446 & 134 & 1298 & 184 & 562.0 & 234 & 272.1 & 284 & 144.8 & 334 & 83.0 \\
\hline 35 & 10580 & 85 & 3375 & 135 & 1275 & 185 & 553.3 & 235 & 268.5 & 285 & 143.1 & 335 & 82.2 \\
\hline 36 & 10320 & 86 & 3306 & 136 & 1252 & 186 & 544.8 & 236 & 264.9 & 286 & 141.4 & 336 & 81.3 \\
\hline 37 & 10070 & 87 & & 137 & 1230 & 187 & 536.4 & 237 & 261.4 & 287 & 139.8 & 337 & 80.4 \\
\hline 38 & 9823 & 88 & 3171 & 138 & 1208 & 188 & 528.2 & 238 & 257.9 & 288 & 138.2 & 338 & 99.6 \\
\hline 39 & 9586 & 89 & 3105 & 139 & 1187 & 189 & 520.1 & 239 & 254.5 & 289 & 136.6 & 339 & 78.8 \\
\hline 40 & 9356 & 90 & 3041 & 140 & 1166 & 190 & 512.2 & 240 & 251.2 & 290 & 135.0 & 340 & 78.0 \\
\hline 41 & 9132 & 91 & 2979 & 141 & 1146 & 191 & 504.5 & 241 & 247.9 & 291 & 133.4 & 341 & 77.2 \\
\hline 42 & 8914 & 92 & & 142 & 1126 & 192 & 496.9 & 242 & 244.6 & 292 & 131.9 & 342 & 76.4 \\
\hline 43 & 8702 & 93 & 2860 & 143 & 1106 & 193 & 489.6 & 243 & 241.4 & 293 & 130.4 & 343 & 75.6 \\
\hline 44 & 8495 & 94 & 2802 & 144 & 1086 & 194 & 482.0 & 244 & 238.3 & 294 & 128.9 & 344 & 74.8 \\
\hline 45 & 8292 & 95 & 2745 & 145 & 1068 & 195 & 474.7 & 245 & 235.2 & 295 & 127.4 & 345 & 74.1 \\
\hline 46 & 8094 & 96 & 2690 & 146 & 1050 & 196 & 467.6 & 246 & 232.2 & 296 & 125.9 & 346 & 73.3 \\
\hline 47 & 7901 & 97 & 2636 & 147 & 1032 & 197 & 460.6 & 247 & 229.2 & 297 & 124.5 & 347 & 72.5 \\
\hline 48 & 7714 & 98 & 2583 & 148 & 1014 & 198 & 453.7 & 248 & 226.2 & 298 & 123.1 & 348 & 71.8 \\
\hline 49 & 7532 & 99 & 2532 & 149 & 996.5 & 199 & 447.0 & 249 & 223.3 & 299 & 121.7 & 349 & 71.1 \\
\hline 50 & 7355 & 100 & 2482 & 150 & 979.5 & 200 & 440.4 & 250 & 220.4 & 300 & 120.3 & 350 & 70.4 \\
\hline
\end{tabular}

Fig. 3

For temperature controls, thermistors are ideal because simple electrical or electronic circuits can be made to give great sensitivity and accuracy. Constant-temperature baths can be controlled to $0.001^{\circ} \mathrm{F}$ easily and to $0.0001^{\circ} \mathrm{F}$ with a little more difficulty. Even finer control can be obtained, but the equipment becomes quite complicated. Of course, it must be remembered that the constant-temperature baths to this precision require good design of the bath itself and that no control, no matter how sensitive, can make up for poor design of the controlled chamber or bath.

Small, disc-type thermistors are now being used in the windshields of aircraft to control the amount of heating applied to the glass to prevent frosting. The thermistor is embedded in the plastic between the layers of 
glass in the safety glass used for windshields, and small wires are brought out to the control circuit. Many glass-coated, bead-type thermistors are being used for controls of aircraft cabin temperature. Here, high speed of response is required, and small beads with a time constant in still air of about 10 sec are used in a high-speed air stream for which the time constant is less than one second. Since such small beads have small currentcarrying capacity without self-heating, it has been found desirable to use 6 or 8 thermistors in a series-parallel arrangement so they will carry 6 or 8 times as much power, but will have the same time of response because each individual unit is exposed to the air stream in the same manner. Also, by using several units in a group, it is possible to select the units in the group so the overall network has a very close tolerance of resistance and temperature coefficient. Such groups can be selected to tolerances of $1 \%$ without much more cost than the thermistors alone, which have individual tolerances of 20 or $30 \%$.

There are 2 basic ways in which thermistors can be used to compensate electronic circuits for variations due to ambient temperature. One is the "individual circuit" compensation method and the other is the overall "black box" compensation method. In the first method, an individual circuit element may have a temperature coefficient which causes changes in the operation of the circuit with ambient temperature changes. A thermistor can be designed to mount in the circuit near such an element so it will respond to the same ambient changes, and it can be connected in the circuit in such a way as to compensate exactly for the changes caused by the original element. For example, it might be desired to have a relay operate at the same voltage over a broad temperature range. Since the copper coil resistance increases with temperature, the actual voltage required to trip the relay also increases with the temperature of the coil. By embedding a small bead thermistor in the coil and connecting it in series with the coil, it is possible to have a network resistance which is nearly constant with temperature, so the relay will pull in with the same voltage applied to it regardless of temperature of the coil. This compensation works whether the coil temperature is a function of the ambient temperature or is produced by self-heating of the coil due to operating current.

In the black box method of compensation, a complicated electronic circuit is supposed to give a certain output when some fixed input is applied to it. Often the output of such a circuit will be a function of ambient temperature. By plotting the variation of the output with respect to temperature, it is usually possible to design a thermistor or thermistor-resistor network which will exactly compensate for the variation of the output and give a uniform output over a broad ambient range of temperature. Transistor amplifiers have been compensated in this way to give constant gain of the amplifier over a broad range of ambient temperature. The same type of compensation has been successful in magnetic amplifiers, which are notorious for their change in output with ambient temperature. In this case, the thermistor is compensating for variations in the resistance of copper coils and also for variations in the $\mathrm{mu}$ of the magnetic cores. 
In the second group, in which the voltage-current characteristic of thermistors is used, there are many commercial applications now operating successfully. If the voltage-current curve of a bead-type thermistor is plotted, as in Fig. 4, it is found to show an increasing voltage with increasing current until a peak value is reached beyond which the voltage decreases as the current increases.

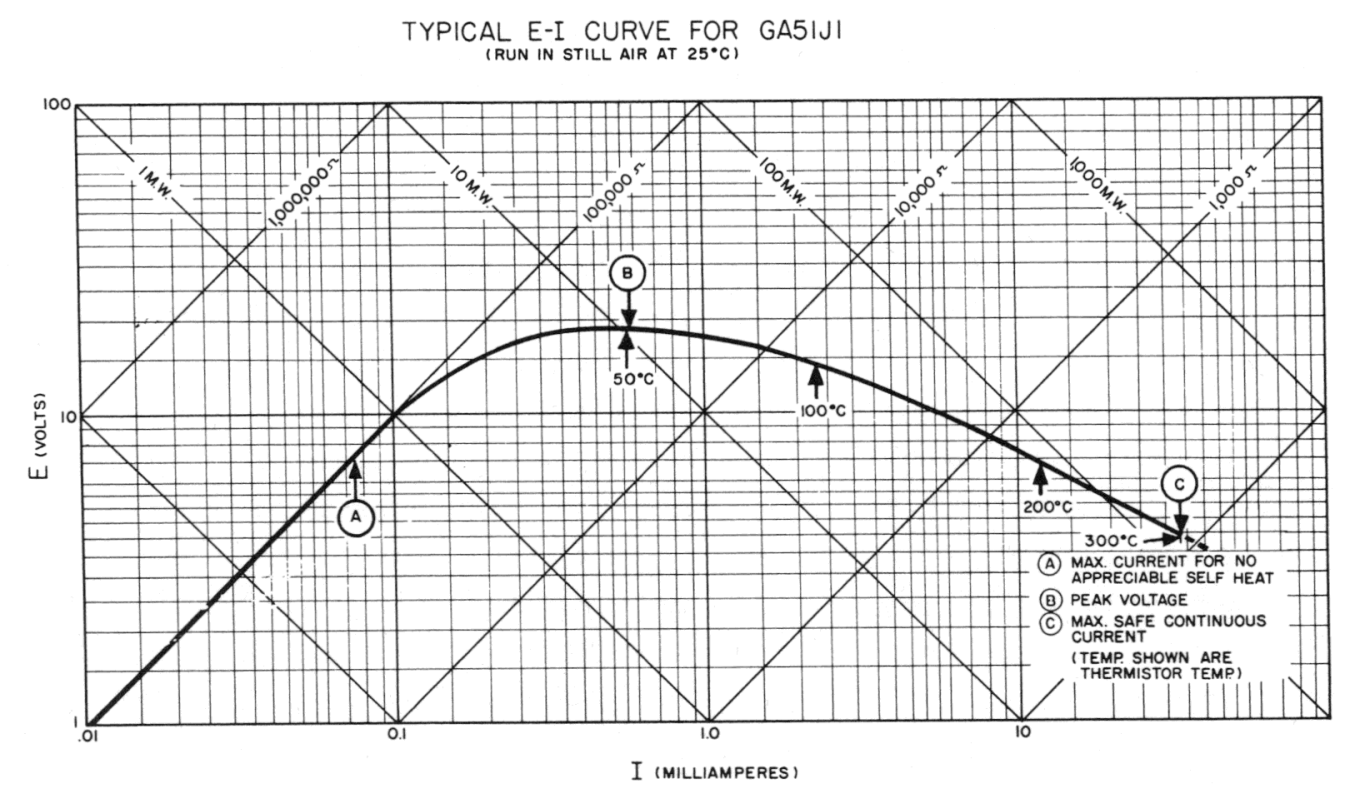

Fig. 4

In this latter portion of the curve, the thermistor is exhibiting a negative resistance characteristic. The slope of this portion of the curve represents the value of the negative resistance of the thermistor and, if an

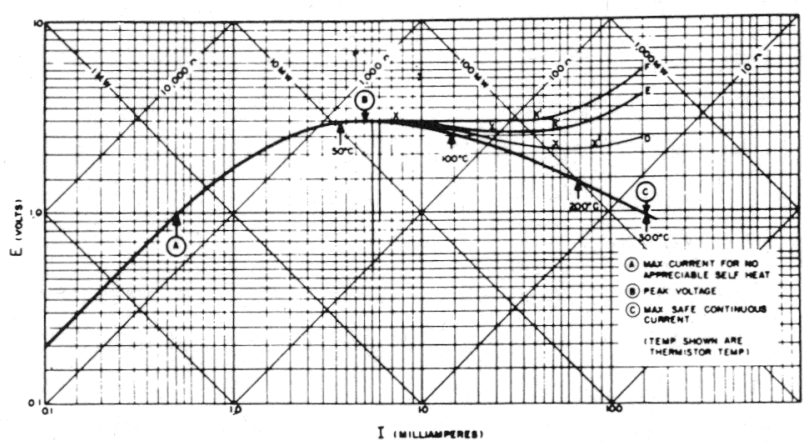

Fig. 5 ordinary resistor of the same value is placed in series with the thermistor, the net result of the 2 elements will be a curve of approximately zero slope over a broad range of current, as shown in Fig. 5. This means that this network will have a constant voltage across it over a broad range of current, and it will therefore be an excellent voltage regulator in an electronic circuit. It can be used in the same type of circuit as a gas discharge tube but its operating range can be as much as ten times that of an ordinary gas tube. A gas tube operates normally over a current range of about 6 to 1 , whereas a thermistor voltage regulator may operate over a range of about 60 to 1 . Such voltage regulators have been used in voltage-regulated power rectifiers where the thermistor network was used to supply a fixed reference 
voltage instead of a battery. This device was used in aircraft where it had to operate over a wide range of ambient temperature and batteries were not very stable under these conditions.

Thermistor voltage regulators are used in oscillators to control the voltage applied to the grid circuit in order to maintain a fixed amplitude of oscillation. Thermistors are particularly good for this purpose because they are strictly resistive and do not cause any phase shift or distortion at moderate frequencies.

One of the largest, single applications for thermistors was in broadband carrier telephone repeaters as controls of power level. In these circuits, a bead thermistor was placed in the negative feedback circuit in such a way that, as the output power level tried to increase, it would reduce the resistance of the thermistor and increase the negative feedback to reduce the gain of the amplifier. The result was output power level control to about one-half $\mathrm{db}$ in spite of very wide variation of input level. In order to hold such close output control, it was necessary to compensate these control thermistors for ambient temperature variation so the resistance would be a function of power only and not ambient temperature. This was done by attaching a small heater to the bead thermistor and applying a small amount of current to it to heat the bead to $120^{\circ} \mathrm{F}$. The amount of current through this heater was controlled by a network of 3 other thermistors and 6 resistors chosen to pass exactly the amount of current required at any temperature to make the bead $120^{\circ} \mathrm{F}$ with no power being dissipated within the bead itself. Under these circumstances, the actual temperature of the bead was then determined by the amount of power in the bead, which was a function of the output power of the amplifier only, since ambient was compensated for automatically. This may sound like a complicated device, but its actual cost was a very small fraction of the cost of controls previously used for this same purpose and it worked much better and gave closer control. These thermistor controls literally saved millions of dollars over the cost of building previously used equipment.

In the third group, that which uses the time-current characteristics of thermistors, as shown in Fig. 6, we find another large application for telephone use. If we apply a fixed potential to a thermistor in series with a resistor, a small current will flow, which will dissipate a small amount of heat in the thermistor. This will raise the temperature of the thermistor and thereby reduce its resistance so more current can flow in the circuit. This heats the thermistor more and lets still more current flow. The resistance of the thermistor keeps getting lower and the current keeps increasing until the voltage drop across the series resistance is high enough to prevent further power increase in the thermistor, at which point the change ceases and the system is in equilibrium. The time required for the current to increase to its maximum value is a function of the resistances of the circuit, the voltage applied, and the dissipation constant of the thermistor. If a relay is placed in series with a thermistor in this way, the current will 
reach a high enough value to close the relay at some time after the circuit is closed. This creates a controlled delay in the operation of the relay after the closing of the circuit. In telephone circuits, the ring-up relays would often close due to some transient disturbance on the line, and the operator would receive a ringing signal. She would answer the line and find it open. When she would pull her plug, the transient set up on breaking the circuit would close the ringing relay on the other end of the line. Much time was lost in this type of false operation. Placing a bead-type thermistor in series with the ring-up relay would cause a delay in its operation of such time that the relay would not close on short transients, but would close in $0.3 \mathrm{sec}$ of the regular ringing signal. This almost completely eliminated false ringing in telephone lines and these thermistors are installed on many telephone lines today for this purpose.
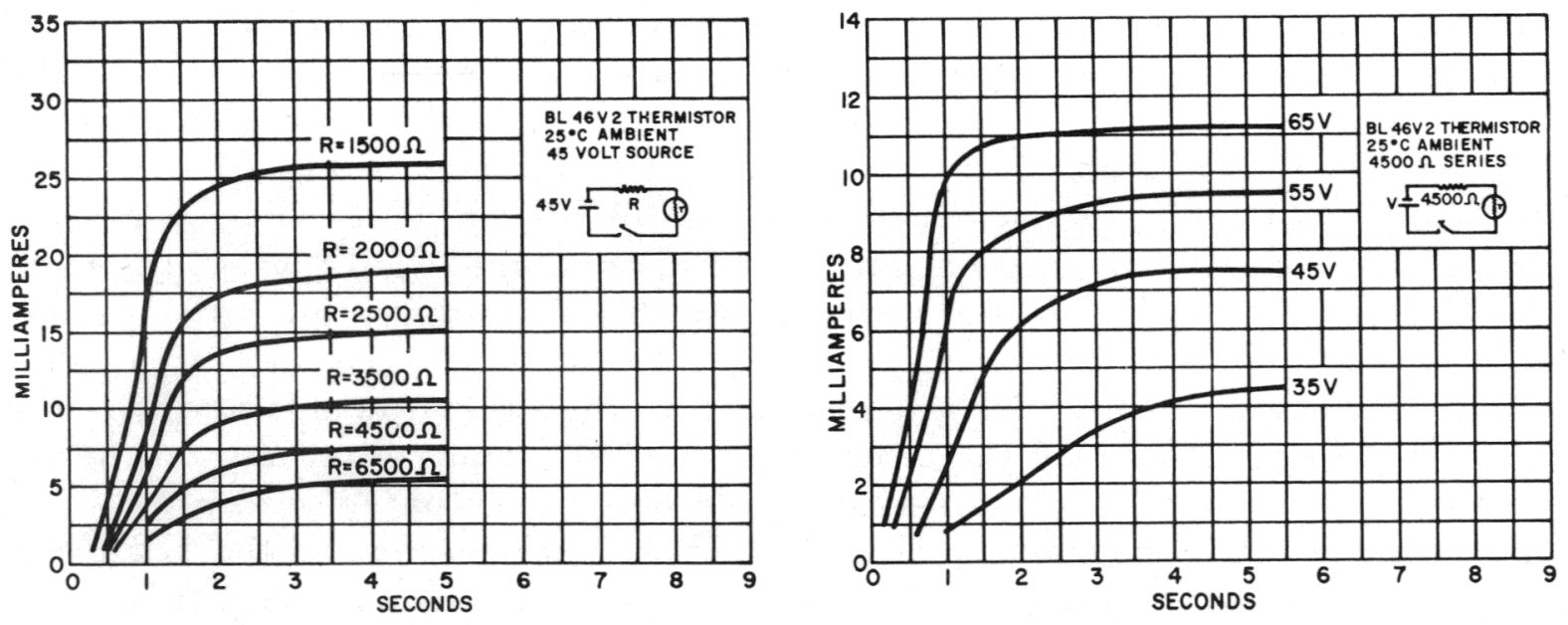

Fig. 6

Since thermistors can be made to have various time delays in circuits, it is possible to build circuits which will integrate or count pulses of power. A thermistor-relay combination can be designed which will not close on 3 pulses of power delivered from a telephone dial but will close on 4 pulses. A whole series of such relays can be set up to operate selectively on various numbers of pulses. In this way, individual circuits may be selected by various numbers of pulses. The operating pulses of power may be supplied from electronic equipment to produce any type of control desired.

If a thermistor in a computer circuit is given a pulse of power, its resistance is reduced according to the size of the pulse. If this same thermistor is given another pulse of power within a given length of time, the resistance can be reduced still further, but if sufficient time elapses between pulses, there is no effect still left from the first one, so the second one will not reduce the thermistor to as low a resistance as it did in the other case. This, therefore, is the basis for a selective circuit which can give a signal as a function of frequency or magnitude, or both, of pulses fed to the circuit. 
This circuit not only has memory of previous pulses, but it also has forgetfulness. It also has stronger or longer memory dependent upon the number of times a certain signal has been repeated.

In the fourth group, which uses physical conditions to change the thermistor resistance, there are many applications. If two bead-type thermistors are mounted in a bridge circuit, as shown in Fig. 7, and sufficient current is passed through them to raise their temperature to some value above ambient like $120^{\circ} \mathrm{C}$ and the necessary

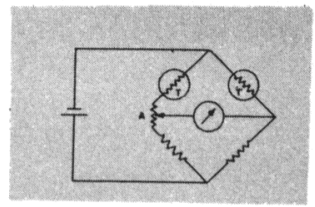

Fig. 7 adjustments are made to make the bridge balance, this circuit can be used as the base of a number of instruments for measuring various physical phenomena. Suppose one of these thermistors is mounted in a wave guide in such a way that it can absorb all of the microwave power being transmitted along the wave guide. If the bridge is balanced with DC and no microwave power is turned on, it is possible to calculate the exact power in the thermistor by measuring the current in the bridge and knowing the resistance to which the bridge is balanced. If, then, the microwave power is turned on, the thermistor absorbing it will be heated and the bridge will become unbalanced. If the DC current is then reduced until the bridge is in balance again, the new DC power in the thermistor can be calculated and the difference in the two values is the amount of microwave power in the wave guide. This circuit and various convenient modifications to it are the major means of measuring microwave power today.

If we take the same circuit but mount both of the thermistors in little holes in a block of metal and put air in both holes, we can balance the bridge. If the air is removed from one of the holes and it is replaced with some other gas such as carbon dioxide, the rate at which heat is conducted away from that bead will be less than it was with air, and the temperature of the bead will rise until all of the power being dissipated in the thermistor is being conducted away by the carbon dioxide. The bridge will now be unbalanced because one thermistor will be hotter than it was before. The amount of unbalance of the bridge then represents $100 \%$ of carbon dioxide in the hole. If a mixture of $50 \%$ of air and $50 \%$ of carbon dioxide is put in the hole, it will be found that the unbalance of the bridge will be just half of what it was with $100 \%$ of carbon dioxide. We, therefore, have an excellent gas analyzer that can be calibrated directly in percentage of mixture of any 2 known gases. A number of such gas analyzers are now on the market for various purposes. This method has been used for measuring the carbon dioxide in flue gases for adjusting furnaces to optimum operating efficiency. It has also been used for adjusting internal combustion engines to maximum operating efficiency by analyzing the exhaust gases. This type of instrument is now being used to measure the percent of oxygen in oxygen tents and in baby incubators. It is also being used to measure the percent of various gas mixtures in chemical-processing equipment in a number of chemical plants. 
If this same bridge is used with one thermistor mounted in a hole in a block of metal and the other one placed inside a pipe that has either a gas or a liquid in it, the bridge can be balanced when there is no flow in the pipe. When flow is produced in the pipe, the greater rate of speed of the fluid past the thermistor will take away heat faster and the thermistor will be cooled and the bridge will be unbalanced. Such an instrument can be calibrated in terms of flow velocity or of flow volume. One nice feature of this type of flowmeter is its broad operating range. The same bridge can be made to operate over a flow range of over 100,000 to 1. If one of the thermistors of this bridge is not placed in a pipe but is mounted up in the air where it is unshielded, it will make an excellent anemometer which can measure very low as well as very high wind velocities. Gas-flow meters such as this have been made to measure flow rates as small as $0.001 \mathrm{cc} / \mathrm{hr}$. By special arrangement of the thermistors in such a flowmeter, it can be made directional, so it will indicate the flow rate and direction in a pipe.

Flowmeters of this type have been used to make very sensitive rateof-climb and rate-of-descent meters for use on aircraft. If one of these directional flowmeters is connected by a small pipe to a reservoir of 1 - or 2 -quart capacity, there will be a flow of air in or out of the reservoir as the aircraft descends or rises. The rate of flow will be proportional to the rate of descent or rise of the plane.

If two thermistors in a bridge circuit are mounted in a tank so that one of the beads is above the other and the liquid level in the tank is rising, when the level reaches the lower thermistor, it will be cooled by the greater thermal conductivity of the liquid than the air and the bridge will be unbalanced. This bridge can be made to operate a control which will shut off the flow of liquid into the tank until the level drops to the point at which both thermistors are again in air and the bridge balances and causes the valve to open and start raising the liquid level. This type of liquid level control can be made to operate to about 0.0001 in. By proper circuitry, it is possible to make such controls operate in $0.01 \mathrm{sec}$ after the liquid touches the thermistor or after the thermistor is removed from the liquid. Level alarms of this type are now being used in aircraft fuel cells to indicate when the cells are full and when they are empty. This is especially important when refueling in flight, because it is essential that fuel be not allowed to overflow a cell because of the fire hazard. The big advantage of this type of level indicator over other types is that it has no mechanical or moving parts to stick or go wrong. The thermistors are permanently mounted in the fuel cell and they are relatively indestructable.

If we use the same bridge circuit and mount one of the thermistors in a glass tube that is then evacuated to a high vacuum and the other one in a glass tube that can be connected to a vacuum system, we can make a very sensitive vacuum gauge which is good over the range of about $10^{-1}$ to $10^{-5} \mathrm{~mm}$ of mercury. The bridge must be balanced with both thermistors 
in a high vacuum; then, as air is let into the vacuum system, the thermistor in it will be cooled more by the small amount of air that is present than the one that is in the high vacuum. The amount of unbalance of the bridge can be calibrated directly in pressure.

Another rather recent development in which a thermistor is used for pressure measurement is the hypsometer. This is a device for measuring the pressure of the upper atmosphere in order to establish the altitude of a high-flying radiosonde. The present hypsometer which was developed for the Signal Corps can measure altitude from sea level to $125,000 \mathrm{ft}$. It consists of a small glass tube with a bead thermistor mounted in it and a small wire heater wound around some insulating material near the bead. About $2 \mathrm{cc}$ of a liquid is placed in the tube and some electrical power is applied to the heater. The heat makes the liquid boil and the resistance of the thermistor is determined by the boiling point of the liquid. Since the boiling point is a function of the atmospheric pressure, the resistance of the thermistor can be telemetered to the ground and interpreted into accurate information as to the altitude of the radiosonde. As the liquid is boiled away, it is recondensed in a condenser above the heater and it runs back into the reservoir for reuse. Two cc of liquid will last through a flight for $3 \mathrm{hr}$ from sea level to $125,000 \mathrm{ft}$.

Previous methods of measuring altitude involved the use of a sensitive aneroid barometer which moved a switch from one position to another to indicate changes of pressure. At high altitudes, the barometer is accurate to about one millibar out of four, which is about $25 \%$. At the same altitude, the hypsometer is accurate to about $1 \%$ of the pressure reading, which is about 25 times as good as the barometer. The hypsometer can be built very small and has a response time of the order of one millisecond.

These are only a few of the many interesting applications of thermistors, and I could go on for hours describing others which have been developed successfully. Thermistors can be used in some way in almost every manufacturing process, and they can be used in almost any electrical or electronic equipment. The main limitation to date in the use of thermistors is the limitation of knowledge of engineers who must design them into equipment in order to establish a new use. Electronics courses in the universities are not teaching new engineers how to use thermistors along with other circuit components, so until they do, it must remain with those few who have had the good fortune to become acquainted with this versatile device to investigate and make use of the almost limitless applications of thermistors in industry. 
Bibliography

Anderson, J. C., Temperature Measurement with Thermistors, Electronic and Radio Engineer (March 1958).

Batcher, Ralph R., Thermistors in Electronic Circuits, Electronic Industries ( Jan 1945).

Becker, J.A., Green, C. B., and Pearson, G. L., Properties and Uses of Thermistors - Thermally Sensitive Resistors, Electrical Engineer ing (Nov 1946).

Bennett, Frank, Designing Thermistor Temperature-correcting Networks Graphically, Control Engineering (Nov 1955).

Benedict, R. P., Thermistors vs Thermocouples, Electrical Manufacturing (Aug.1954).

Benson, G. W., Cowan, C. B., and Stirling, P. H., A Theoretical Treatment of the Sensitivity of Katharometers, Central Research Laboratory, Canadian Industries Limited, McMasterville, Quebec.

Bollman, J.H., A Pilot-channel Regulator for the K-l Carrier System, Bell Laboratories (June 1942).

Dowell, K. P., Thermistors as Components Open Product Design Horizons, Electrical Manufacturing (Aug 1948).

Gaffney, F. J., Microwave Measurements and Test Equipments, Proc. Inst. Radio Engrs.,(Oct 1946).

Goodyear, R. S., Thermal Resistor Elements for Electrical Circuit Application, Product Engineering (Feb 1945).

Green, E. I., Fisher, H. J., and Ferguson, J. G., Techniques and Facilities for Microwave Radar Testing, A.I.E.E. Technical Paper 46-40 (Jan 1946).

Hauck, V.D., Cosby, J.R., and Dember, A.B., Radiosonde Telemetering Systems, Electronics (May 1946).

Montgomery, C.G., Techniques of Microwave Measurements, M.I.T. Radiation Laboratory Series, McGraw-Hill, Chap. 3, "Use of Thermistors."

Pearson, G. L., Thermistors, Their Characteristics and Uses, Bell Labs. Record (Dec 1940). 
Sachse, H. B., Thermistors as Sensing Elements for Low Temperatures, Kaystone Carbon Co., St. Mary's, Pa.

Weller, J. A., A Volume Limiter for Leased-line Service, Bell Labs. Record (March 1945).

\section{Discussion}

\section{THERMISTORS}

W. L. Donaldson, Southwest Research Institute: I am interested in a dynamic application of that bridge. What frequency response at the high end do you think you get with the best available thermistors?

R.S. Goodyear: The time constant on a small, bead-type thermistor is about $0.7 \mathrm{sec}$. However, these can be put in an helium or hydrogen atmosphere, which greatly increases the response; they probably follow 20 cycles. There is, however, another type of thermistor which has been developed which would self-oscillate at $70 \mathrm{kc}$. This type is not currently under manufacture. The flake-type thermistors which are used for radiation bolometers will oscillate at or follow a thousand cycles. tur e limit?

H. E. Bömmel, University of California: What is the low tempera-

$$
\text { R.S. Goodyear: The ones that we use have a resistance too high }
$$
to be measurable below $100^{\circ} \mathrm{C}$. Below that, the resistance goes up to $10^{15}$ or $10^{16}$; this isn't a very good device. However, there are thermistors available which go down to the neighborhood of $0^{\circ} \mathrm{K}$ and are actually being used in liquid helium. 
T. H. Blewitt

Argonne National Laboratory

Argonne, Illinois

\begin{abstract}
The reactor irradiation of solid materials results in the formation of lattice defects. At sufficiently low temperatures, individual vacancies and individual interstitial atoms are probably the only defects formed; however, near room temperature in many materials these defects have migrated and coagulated resulting in small dislocation-loop formation. All of these defects substantially effect the mechanical properties of solids, e.g., the yield stress of copper can be raised by a factor of 40 by a moderate bombardment. The correlation of this change with measurements of physical properties is extremely poor, however, probably due to the fact that mechanical properties are strongly dependent on point defect-glissile dislocation interactions which are difficult to measure by other means. The various physicalproperty measurements - electrical resistivity, elastic constants and logarithmic decrement, stored energy, and volume expansion and the influence of nuclear irradiation on them will be discussed together with the mechanical property measurements.
\end{abstract}

(The manuscript of this paper is not available.) 


\title{
COLOR RADIOGRAPHY
}

by

Norman S. Beyer

Special Materials and Services Division

Argonne National Laboratory

Argonne, Illinois

\begin{abstract}
Notwithstanding those intangible aspects of any development project which attract investigators, the physiological fact that the human eye is more sensitive to changes in color than to changes in brightness makes a study of the production of color radiographs attractive. Some color radiographs and autoradiographs together with the technique used to produce them are presented. An evaluation is made of both the practical and impractical aspects of the study of date.
\end{abstract}

\section{Introduction}

It is a physiological fact that the human eye is more sensitive to color differences than to different shades of gray. Stimulated by this information, we initiated a project to study the production of color radiographs. This paper reports on the status of the project which has been in progress about 4 months. The material includes a discussion of a justification for the study of production of color radiographs, an explanation of what we designate as a color radiograph, and the presentation of some recent results with comments concerning them. The reproductions which are presented here may be grouped as follows: large-hue difference examples, welds, high-latitude subjects, internal homogeneity presentation, and crack location. Black and white radiographic counterparts are presented for some cases. Each case is discussed briefly concerning its production, possible application, and its qualities which are likely to direct future developmental work. The presentation is intended only as a status report. Only a superficial effort is made to explain the fundamentals of the mechanism of the production of the color radiographs. Our primary purpose at this time is to present some results for examination.

\section{Discussion}

A justification for the study of the production of radiographs in color can be made by considering the visual attributes of color. Figure 1 is a 


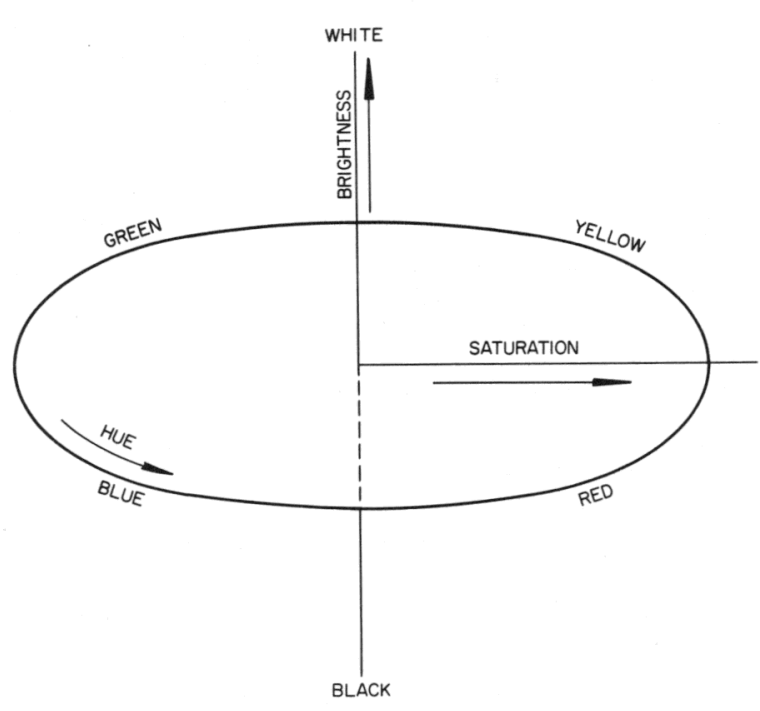

Fig. 1. Visual Attributes of Color diagrammatic representation of these attributes. One visual attribute of color is its degree of brightness. The vertical axis in the diagram is intended to represent various values of color brightness perceived by the eye. It ranges from black at the lower end to white at the upper with varying shades of gray in between. The information contained on a conventional black-and-white radiograph is restricted to this single dimension. In other words, when studying a conventional radiograph, only differences in gray may be interpreted as the differences of the structure of a material. A second attribute of color perception, and the one learned early in childhood, is difference of hue. It is simply the condition of the object which we perceive as red, blue, green, etc. Hue is designated in the diagram by a particular angle of rotation made about the brightness axis. It is measured in some isobrightness plane which is perpendicular to the brightness axis. A third attribute of color perception is that of saturation. Saturation is that condition perceived as a redder red, greener green, etc. It is presented on the diagram as a radial distance from the brightness axis. These three stimuli: brightness, hue, and saturation, should each contribute information about an object radiographed in color. As mentioned earlier, conventional black-and-white radiographs display information only by brightness differences. The possibility that a color radiograph is capable of containing more information due to the addition of the 2 parameters hue and saturation gives justification to the study of the production of color radiographs.

Some comments on the nature of our radiographs and the method of production should clarify what we are designating as a color radiograph. The color radiographs we have produced were made on conventional multilayer, commercially available color film, such as Ektachrome or Ektacolor. A typical three-emulsion layer color film is illustrated in Fig. 2.

Fig. 2

An Exposure Technique for Producing a Color Radiograph

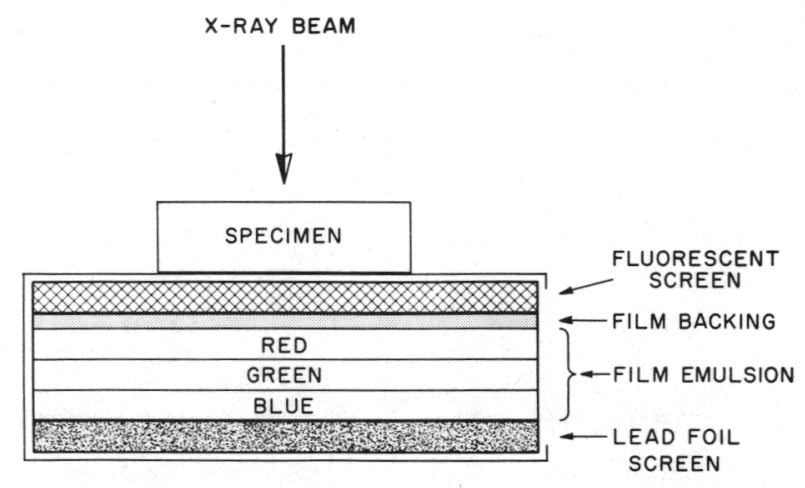


We used conventional $\mathrm{X}$-ray machines and radioactive isotope sources. Our radiographs do not nor are they intended to display color differences as normally seen by reflected visible light. We believe the hue, saturation, and brightness differences primarily indicate differences such as thickness, density, and type of material. Variation in appearance with variation in transmitted energy of the radiation has not been uniquely observed to date. X-ray energies ranging from $17 \mathrm{kvp}$ to $250 \mathrm{kvp}$, and gamma sources of cobalt-60 and cesium-137 were used. One of the most successful techniques we have used to produce large hue differences is an adaption of a method described by Bryce.(1) The successful technique reported by Blais (2) has also been adapted in part.

Figure 2 is a diagram of one arrangement we used to expose the color film. A fluorescent intensifying screen and a lead screen are used as shown. The positions of the screens shown in the figure may also be reversed, depending on the result desired. Conventional radiographic calcium tungstate screens have been used primarily; however, screens of other phosphors are under study. After the film has been exposed to the beam of radiation, it is then processed by ordinary techniques prescribed by the film manufacturer except for one additional step. The additional step amounts to an interruption of the processing after partial development in the first developer. At this point the film is exposed to colored light. Normal processing is then completed. At the present time, exposures to either red or green light have been the most satisfactory. It is believed that the image brought out in the top emulsion layer after partial development is printed on the lower 2 layers by the exposure to colored light. The combination of the various hues and degrees of brightness and saturation produced in each layer by the screen fluorescence, X-ray beam, and colored-light exposure combine to form the resultant differences on the color radiograph. The flashing technique was used for all the cases shown except for the penetrant-color radiographic technique employed for crack location.

\section{Results}

Reproductions of some of the color radiographs we have obtained are presented in Figs. 3 to 11 . Figures 12 to 15 are conventional blackand-white radiographs and are included for comparison. A short discussion of each of the color figures is given. It is not feasible at this time to present a thorough discussion of all the factors contributing to their production.

The purpose of showing the foil radiographs reproduced in Figs. 3 to 5 was to present some examples of the greatest hue differences obtained to date. Figure 3 is a reproduction of a radiograph of 4 uranium foils. The square image was from a foil $0.001 \mathrm{in.}$ thick, and the circular images were each from foils 0.002 in. thick. By a staggered piling arrangement, 
images of $0.001,0.002,0.003,0.004,0.005$, and 0.006-in. thicknesses were obtained. The large hue difference between 0.001 and 0.003 -in. thickness is interesting to note. The hue difference between 0.002 in. and $0.003 \mathrm{in.}$ is also significant.

The radiograph of Fig. 3 was made by exposing the film in the type of cassette arrangement shown in Fig. 2. For this case, a conventional, medium-speed, calcium tungstate fluorescent screen was used. During the processing of this exposed film, the first development was interrupted approximately midway and the emulsion side of the film flashed to red light. Typical of this technique is the predominance of the hue of the colored-light flash exposure in areas of low X-ray exposure. Figure 4 was produced in the same manner as Fig. 3 except that a green flash was used. The increase in the number of hue differences is apparent.

Included in the exposure of Fig. 4 was an aluminum step wedge of $\frac{1}{4}, \frac{1}{2}$, and $\frac{3}{4}$-in. steps. Placed on the wedge near the lead numeral 8 is a tungsten ribbon, $0.040 \mathrm{in.}$ wide by $0.001 \mathrm{in}$. thick. Following the ribbon are tungsten wires of $0.001,0.008,0.010$, and $0.012-i n$. diameters. The 0.001 -in. wire was clearly visible on the original radiograph. The side marked $8 \mathrm{~B}$ designates a slightly longer exposure than 8A.

Figure 5 shows three different exposures of the above foils and wedge. Radiographic sensitivity is demonstrated for this case by the images of the ASME aluminum penetrameters. A wedge of 0.002-in. steps of zirconium was also included in one shot to study hue changes. The technique for producing Fig. 5 was the same as the previous case except both sides of the partially developed film were flashed to green light. The duration of flash was approximately halved. Since such large hue changes can be shown for 0.001 -in. thickness differences, the possibility of using color radiographs of foils to detect smaller and otherwise difficult to measure variations of thickness by hue change should be considered as a possible application.

An example of color radiography of welds is presented in Fig. 6. These were purposely poorly made welds of $\frac{1}{2}$-in. aluminum plate. A single green flash directed at the emulsion side was used. The aluminum step wedge with tungsten wires as previously described is included for comparison. Lack of penetration in the weld appears as a yellow hue, and tungsten inclusions are dark brown tending toward green. Two percent or better radiographic sensitivity is displayed on the middle weld plate. The penetrameter was a standard ASME type having a specified thickness of 0.010 in. (i.e. $2 \%$ of 0.500 in.) and holes of $0.020,0.030$, and $0.040-$ in. diameter. 


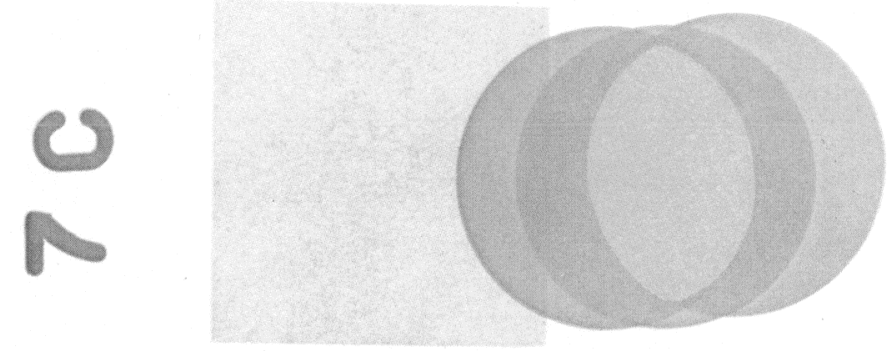

Fig. 3
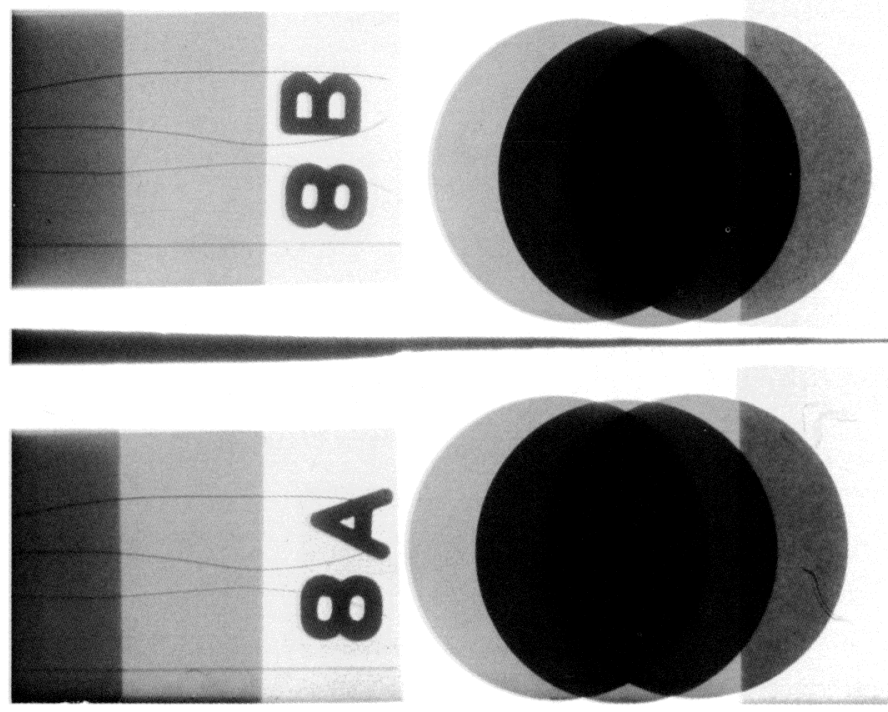

Fig. 4

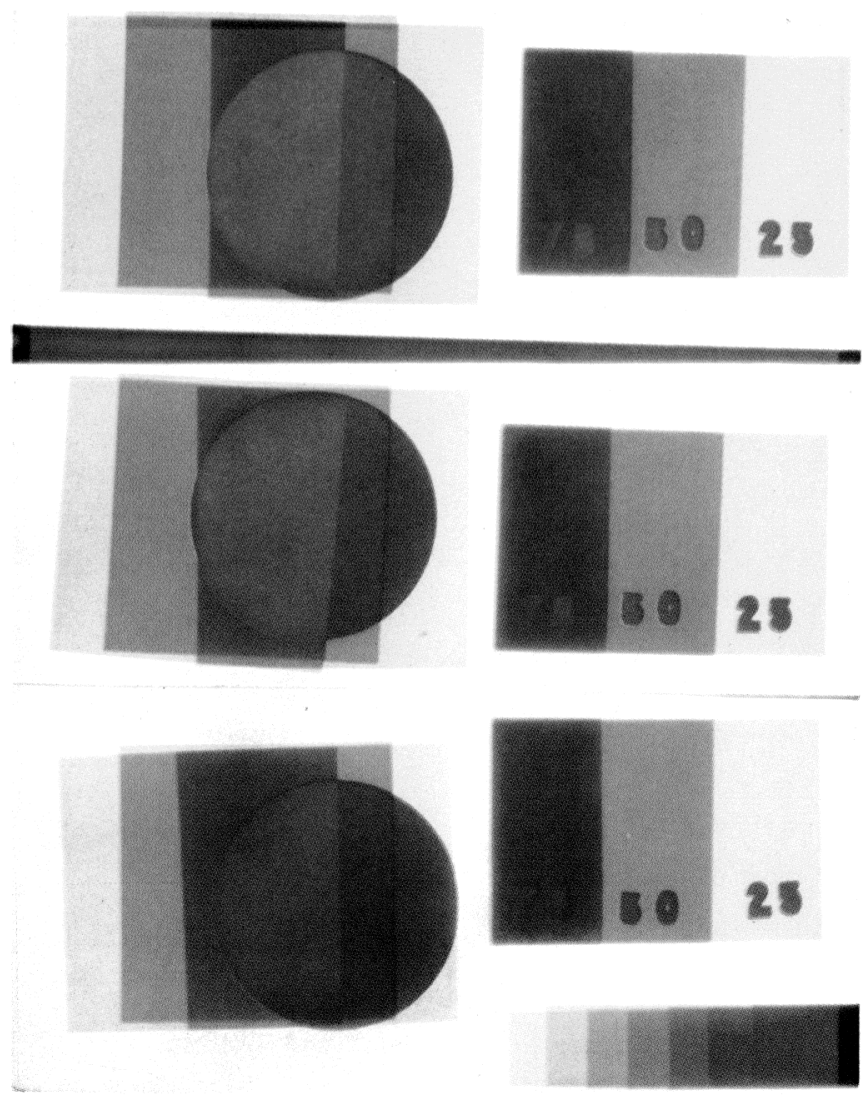

Fig. 5
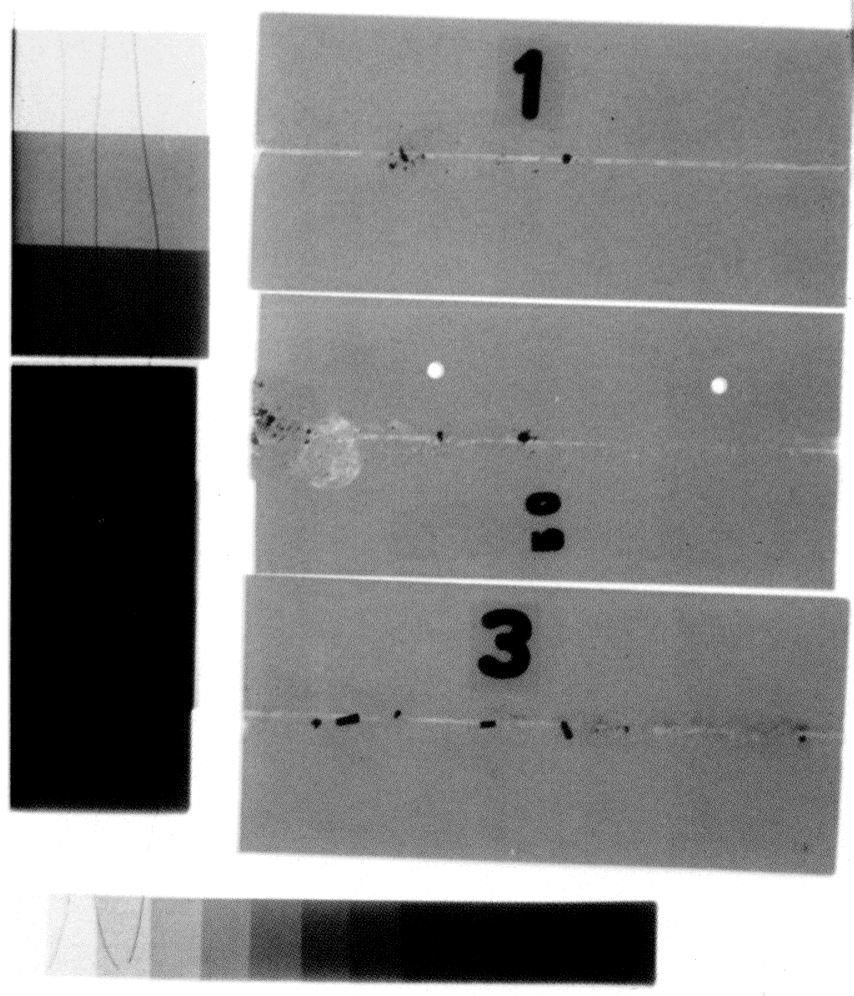

Fig. 6 


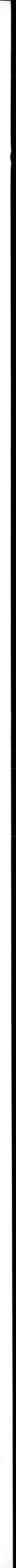


The reader will note from a study of the figures that a greater radiographic latitude (i.e., range of $X$-ray opacity visible on a film) is displayed on the color radiographs than is usually obtained with blackand-white radiography. The range of latitude obtainable with black-andwhite radiographs is restricted to brightness differences. From a quick appraisal, it appears that latitude is extended in color radiography by the addition of hue differences and that good radiographic contrast is preserved. Perhaps it may be even further extended when techniques for analyzing the degree of color saturation are developed.

Radiographic latitude is of course dependent upon the type of film emulsion used. The black-and-white radiographs presented in Figs. 12 to 15 were made on fine-grain, high-contrast films of the type customarily used to display fine detail. They are therefore of low latitude. A study is now in progress to compare the latitude of color radiographs with radiographs made on high-latitude, black-and-white film when equivalent radiographic contrast is maintained for each case. Note that a contrast sensitivity of $2 \%$ or better is possible on color radiographs while still maintaining a high latitude.

Figures 7 and 8 are included as examples of high latitude in color radiography. Figure 7 is almost self-explanatory. The great range of $\mathrm{X}$-ray opacity presented by vacuum tubes or by a conventional test meter presented in Fig. 7 is known to most readers. Figure 8 is a color radiograph of the anode end of a large klystron tube. It was produced using a 250-kvp X-ray beam, a fine-grain calcium tungstate screen, and a single-green-flash processing technique. The klystron was constructed of materials varying in X-ray opacity from a very thin aluminum cap at the narrow end, glass-to-metal seals, copper, and finally heavy sections equivalent to an inch or more of stainless steel. It is interesting to note that details of the interior components are well defined and easily distinguishable throughout the area viewed. Included in the radiograph is the image of a stainless steel step wedge of steps of $\frac{1}{8}$ to 1 in. Other experimental shots have indicated that it is possible to attain greater latitude than displayed here. A radiograph of the above wedge and its companion wedge which extended the steps to 2 in. was obtained with all steps distinguishable.

The inhomogeneous distribution of uranium in a reactor fuel element is displayed by the color radiograph included as Fig. 9. It has been suggested by observers of this example that color radiographs may prove to have practical value because they can be rapidly read. For rapid film reading, dark adaption of the eye seems less important for color than for the case of reading black-and-white radiographs.

A slightly different technique than previously described was used to produce the color radiographs presented in Figs. 10 and 11 . 
We have combined penetrant inspection with color radiography in an effort to develop an improved method for crack location. Compounds which have a predominant hue in their fluorescence were mixed with conventional penetrating solutions and the mixture applied to cracked surfaces. Phosphors with acceptable characteristics (i.e., small and uniform grain size, moderate persistance, etc.) and which were readily available were found to be those produced for color television tubes. By means of a P-22 phosphor (red zinc phosphate), cracks were penetrated and filled on one side of our test specimen. This side of the specimen was then placed in direct contact with the emulsion of the color film. This was of course done in a darkroom environment and placed in a light-tight envelope for exposure to the X-ray beam. The film was processed in the conventional manner. There was no flashing to colored light. Figure 10 shows the resulting radiograph. Cracks coming to the surface are red in appearance. The solid portions of the specimen are dark blue, and other cracks (interior or opposite surface) are lighter blue.

By penetrating the cracks on the opposite surface with a yellowfluorescing phosphor, such as $\mathrm{P}-7((\mathrm{ZnCd}) \mathrm{S}: \mathrm{Cu})$, a second radiograph is produced which displays its surface cracks as a yellow hue. This is shown in Fig. 11. A black-and-white radiograph displays all the cracks as gray images and does not lend itself to easy differentiation of the surface upon which they are located. It is, of course, possible by examining a specimen visually together with its black-and-white radiograph to determine which cracks are on a particular surface. But this one-to-one correspondence method is tedious and time consuming. Obviously, analysis for crack location using color radiographs is much simpler. The location of a surface crack that becomes an interior crack can also be easily accomplished. This is done by noting a crack that changes from surface hue (for example red) to the light blue color of an interior crack. Of course, purely interior cracks can also be located easily by their hue difference. Finally, it should be mentioned that the images of extremely fine surface cracks are more easily observed by the penetrant-color radiograph technique.

Figures 12 to 15 are presented to compare some of the color radiographs with black and white. High-contrast, fine-grain X-ray film was used in all cases. No special comment concerning Figs. 13 to 15 seems necessary except to state that an effort was made to produce examples of high quality. Fig. 12 is a radiograph of the same uranium foils used for the color cases. An exposure technique was chosen which gave reasonable density for the 0.001 -in.-square foil denoted by the numeral 1 on the radiograph. The numerals 2,4 , and 6 below the circular foils refer to thicknesses of $0.002,0.004$ and $0.006 \mathrm{in}$., respectively. A conventional, fine-grain, high-contrast X-ray film was used in order to obtain for comparison the best definition of inhomogeneity in the $0.001-i n$. foil. A higher latitude, but necessarily lower contrast, black-and-white film could have been used to differentiate the thicker foils in a gray scale but the presentation of fine detail would have been sacrificed. 

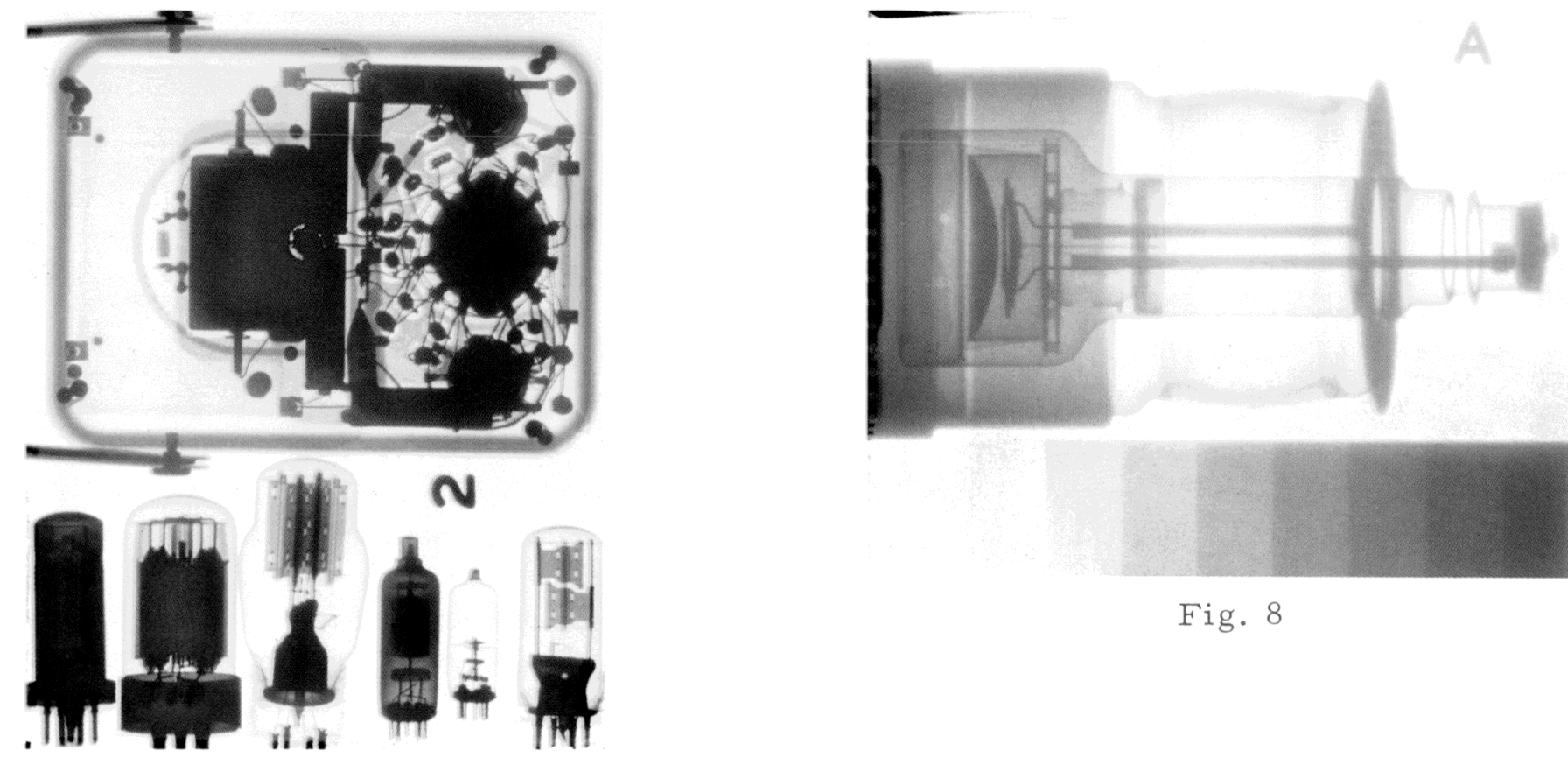

Fig. 7

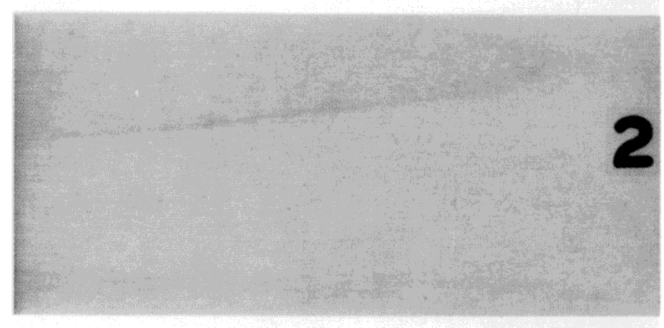

\section{Fig. 8}

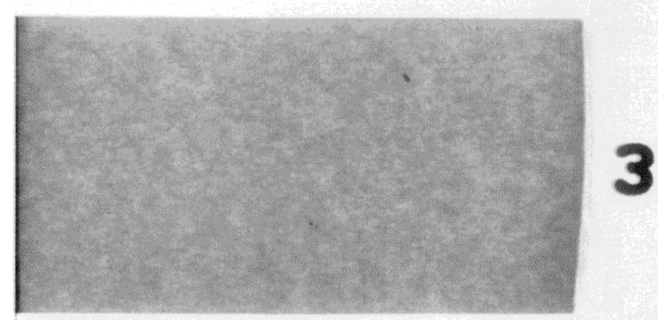

Fig. 9
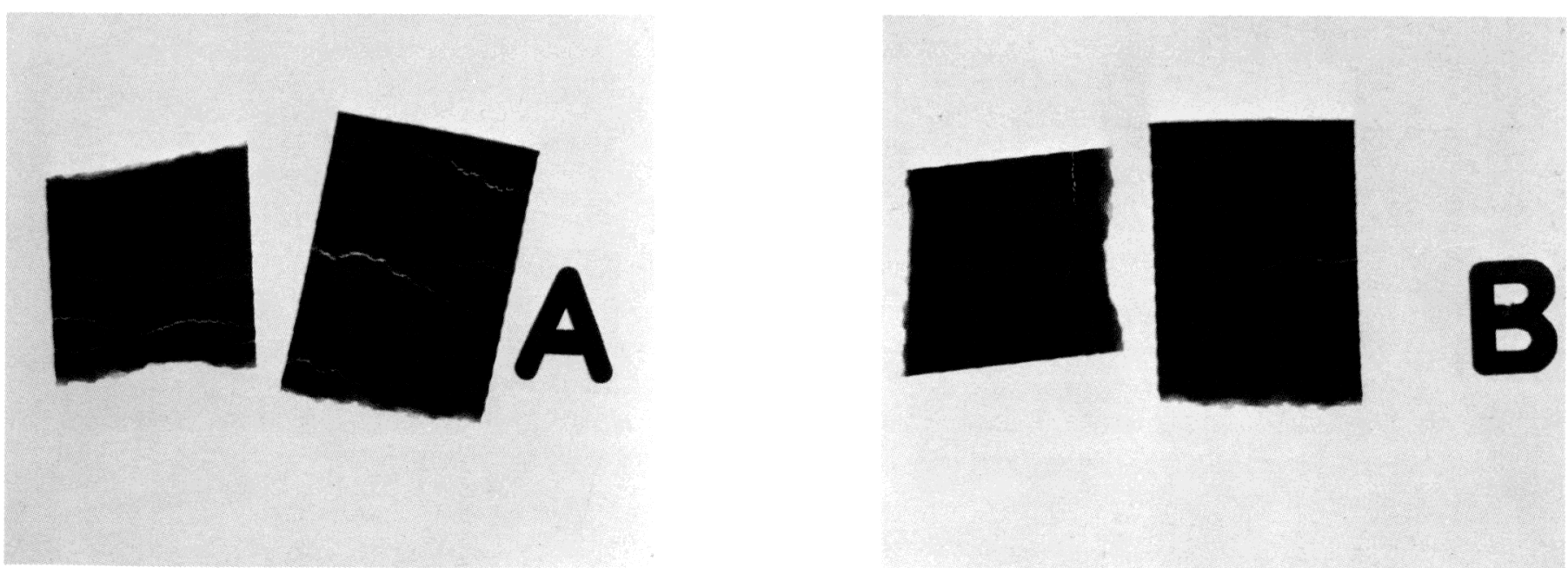


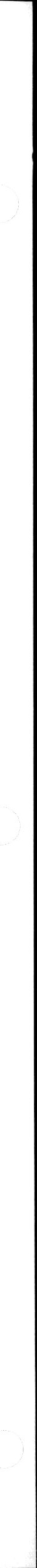




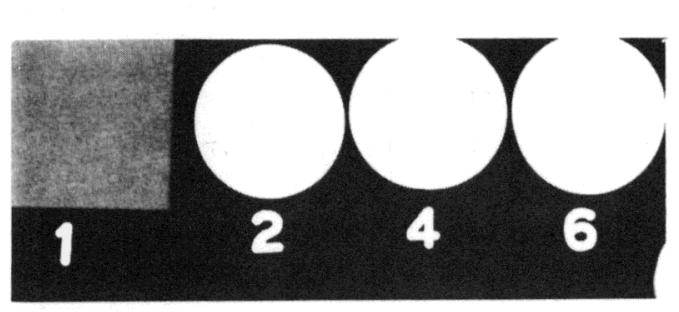

Fig. 12
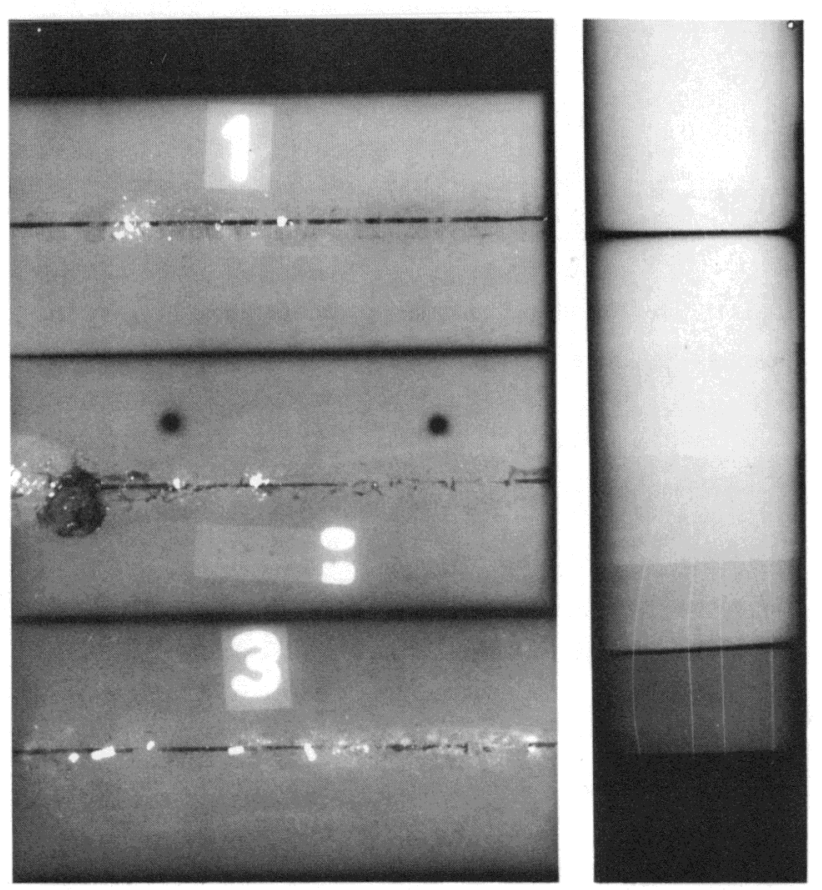

Fig. 13
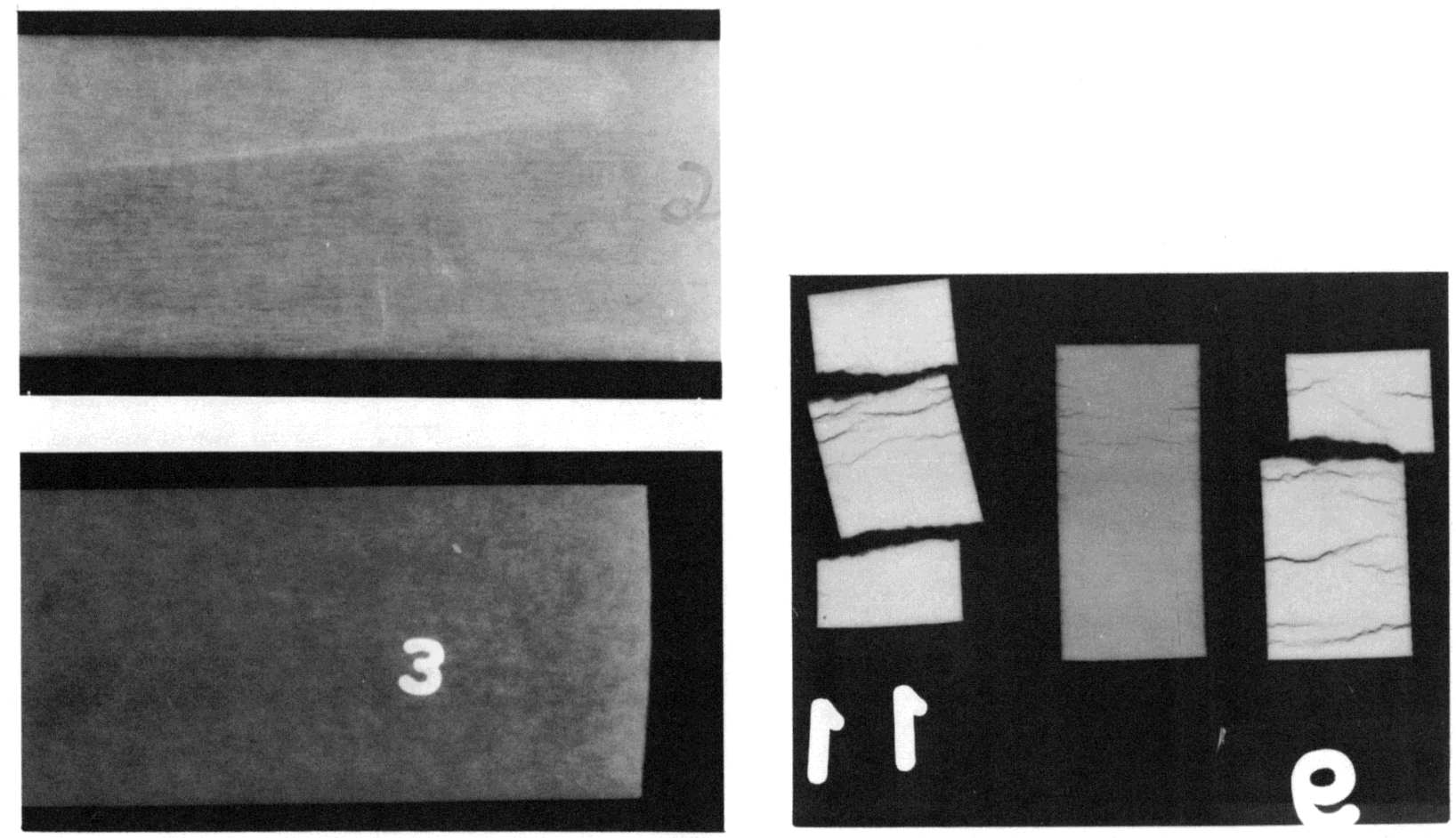

Fig. 14

Fig. 15 


\section{Comments and Conclusions}

The previous discussion has pointed to some ways in which color radiography may find application and complement inspection with blackand-white radiography. Conclusions to be made from the discussion of the results are as follows: color radiography may make possible improved film latitude through hue change, more precise detection of thickness change seems likely, faster film reading has been suggested, and improved crack location is possible.

Future effort might make it possible to evaluate results by proper color designation, by means of some system such as the C.I.E. or Munsell. Such an analysis could make better use of the information presented on the film, and a special film-reading technique for color radiographs be developed. Other developmental work could include the study of different types of screens, special viewing techniques, improved penetrants, different flashing, special processing, different emulsions, autoradiography, energy response, etc.

Bibliography

1. Bryce, M. B., Experimental Color Radiography, Brit. J. Radiol., 28, $552-553(1955)$.

2. Blais, J. M., and Schwerin, A. K., A New Color-Radiographic Process, Radiography, 21, 254 (1955)。(Summary of paper from The Focal Spot, Journal of the Canadian Society of Radiological Technicians, 11 (2), 65$66,1954$.

\section{Discussion}

\section{COLOR RADIOGRAPHY}

E. E. Potter, Commonwealth Edison Co.: What magnitude of exposure times are we talking about to get these beautiful results?

N. S. Beyer: The exposure times are not much different from the exposure times used for a slow, fine-grain, black-and-white film. Of course, color radiography isn't developed to the point where one can take a lot of rapid shots knowing ahead of time a good exposure technique for each shot.

B. Ostrofsky, American Oil Co.: Do you get comparable results with gamma radiography as you do with $\mathrm{X}$ rays? 
N. S. Beyer: Yes, we can get about the same results, but we haven't done much gamma radiography. As I mentioned, we did a few shots with some small cesium sources and a little work with radioactive cobalt.

S. Leonard, Lockheed Aircraft Corp.: Figure 2 showed your technique where you had a fluorescent screen. Was that a typical setup?

N. S. Beyer: That was quite typical. It was used on quite a few of the sides shown, with the exception of the last group of crack radiographs.

S. Leonard: Basically, then, you had 2 radically different methods. One was the regular radiographic approach, and the other was like a contact photograph using $\mathrm{X}$ rays.

N. S. Beyer: Yes, I discussed 2 techniques. Most of the cases exhibiting striking hue differences were obtained by the addition of a colored flash exposure or, as you call it, the contact print technique.

S. Leonard: I would like to return to the method which used the fluorescent screen. I would like to review this to get the principle involved. The screen would expose one of the emulsions. Actually, your $\mathrm{X}$ rays would have exposed all 3 emulsions, but the screen would preferentially expose only one of the emulsions. Then, you would either pre-expose before the $\mathrm{X}$ ray was taken, or make a post-operative exposure. In other words, you are mixing colors.

N. S. Beyer: We are doing some mixing. There was no preexposing. The post-operative exposure, as you call it, was used in some cases.

S. Leonard: It is conceivable, then, that you could slip a filter in behind your screen and filter out certain color components from the screen itself, so that you could get a even more preferential exposure of, say, the blue emulsion.

N. S. Beyer: That is correct. We have done a little work with screens that have different predominant hues when they fluoresce. We haven't done much with filters. We use different phosphors - different screens with a different color spectrum.

S. Leonard: Film reading of just black-and-white radiographs is a science or an art. I can anticipate, though, in reading these color radiographs that you'll need a great deal of education. And, wouldn't there also be a problem with color perception?

N. S. Beyer: There certainly will be. We are adding a lot more variables than there are in the black-and-white situation. But, perhaps, we've obtained some more information too. 
G. M. Corney, Eastman Kodak Co.: In the screen technique, are you obliged to use a film without an antihalation backing, or do you remove the backing yourself before you make the radiograph?

N. S. Beyer: The most satisfactory shots as far as hue difference obtained have been with the antihalation backing in place and adjacent to the fluorescent screen.

G. M. Corney: I would have expected that the antihalation backing would be essentially opaque to the light of your exposure.

N. S. Beyer: Apparently, it is not opaque to everything that comes from the calcium tungstate during fluoresence, but it does cut out a lot. It lets through enough to get favorable results. That's about as far as I can go; we're not prepared at this stage to give you details of the mechanism of the technique.

G. M. Corney: What is the magnitude of your second visible lightprinting exposure?

N. S. Beyer: Two and a half seconds at 36 in. with a number one photoflood lamp filtered by a Kodak series 7 safelight filter is an example of one flashing technique.

D. R. Green, General Electric, Hanford Atomic Products Operation: Have you made any tests to determine the effects of operator fatigue from the examination of black-and-white radiographs as compared with the examination of color radiographs?

N. S. Beyer: No, we haven't made any tests, but it seems probable that in most cases the fatigue will be less when one is reading color radiographs.

F. K. White, U.S. Atomic Energy Commission, Chicago Operations Office: Can you, with a weld overlay, determine the depth of the weld penetration into the base material?

N. S. Beyer: There is a possibility, but first we have to learn to read the films correctly. In the one weld case I showed, the yellow portion of the weld could be compared with the yellow portion of the adjacent step wedge of known thickness. So, you could go this far - you could say that the thickness at that place on the weld is very close to the thickness of the wedge.

F. K. White: Was this using a dye penetrant? 
N. S. Beyer: No, it was not made with a penetrant. We're using penetrants on such things as cracks and holes.

$\frac{\text { F.K. White: }}{\text { dye penetrants? }}$ Are you able to determine the depth of cracks without

N. S. Beyer: No. The use of dye penetrants helps to determine which cracks go interior and their surface location. Perhaps with further study one might estimate the depth at which a crack becomes an interior crack.

E. D. Harvey, Hercules Powder Co.: The different hues that you showed are the result of a change in quantity of $\mathrm{X}$ radiation. Do you also find a change in hue with a quality change of radiation?

N. S. Beyer: We haven't been able to demonstaate any distinct hue changes with energy in the ranges studied. We have worked in the range of about 17 to $250 \mathrm{kvp}$. This is one thing we've been looking for, and we do want to do more of this. As you know, there is a little problem here. You've got to do some careful dosimetry to be sure that the dose is the same for the different energies compared, and you must measure this intensity or dose over a thin layer of film. We have noticed a little effect in the low range. There seems to be a little enhancement of hue difference in the low-kvp range, but I'm not prepared to give an accurate report about this now. We're quite interested, and future work may be directed this way if it doesn't get too complicated.

G. M. Corney: I should like to comment on this question of difference in color with radiation quality; one would not expect much difference in color as you change the radiation quality. The absorption of an emulsion, particularly a color emulsion, is very low at any wavelength. It is less than one percent. This means that all 3 color layers would essentially be exposed no matter what quality of radiation one used. If the layers were all exposed to the same degree, one would get the same net color projected on the screen. This is why I would not expect a color difference with radiation quality.

\section{N. S. Beyer: Thank you.}

G. M. Corney: Did you have the opportunity to make a comparison black-and-white radiograph of the klystron tube at a high enough voltage to give you equivalent subject contrast, or on a black-and-white material of low enough contrast to give you an equivalent radiograph to your color shot?

N. S. Beyer: We were unable to go to a higher voltage to obtain higher latitude. However, we tried some shots using $F$ film for the increased latitude. We plan to try some shots using the highest latitude black-and-white film available. By comparison with the color shots, we should then be able to tell whether hue difference gives us more latitude without the equivalent loss of contrast. It does appear, however, that the latitude is extended by the addition of hue differences. 


\title{
SOME DESIGN ASPECTS OF LINEAR ACCELERATORS USED FOR HIGH ENERGY X-RAY PRODUCTION
}

$$
\text { by }
$$

J. Haimson

Radiation Division

Varian Associates

Palo Alto, Cal.

\begin{abstract}
A brief description of the theory of operation of the linear accelerator (linac) is followed by a discussion of some fundamental design parameters and their relationship to electron and X-ray beam performance. By utilizing an electron beam bending system and rotating X-ray head, the X-ray beam may be directed at right angles to the accelerator waveguide and rotated through $360^{\circ}$ in a vertical plane. This feature, which provides greater operational flexibility and extreme X-ray source stability, depends upon the achievement of a stable and sharp electron energy spectrum and a small beam cross section. These requirements are related to several aspects of linac electron beam optics and some examples are given.
\end{abstract}

\section{$\underline{\text { Introduction }}$}

Several papers have been published $(1-5)$ dealing with the general theory and operation of the linear accelerator. The brief description below serves only as an introduction; for more specific details the reader is referred to the above references.

\section{General Theory of Operation of Linac}

In general, the linac waveguide through which the electrons are accelerated comprises a straight length of a copper waveguide of circular cross section divided into a series of "partial cavities" by the insertion of annular discs at various intervals (see Figs. 1 and 2). Radiofrequency power coupled into a loaded waveguide of this description can be conveyed along the structure in a variety of modes and, by suitably shaping and locating the annular discs, the axial electric fields, and the phase and group velocities of the rf waves can be accurately controlled. One form of design arranges for the rf phase velocity in the initial portion of the waveguide to 
match the velocity of the electrons which are injected into the structure from an electron gun. Through an initial sorting and then bunching process, electrons become trapped by the electric fields and bound to the rf wave, the velocity of which is then arranged to increase up to the speed of light, thereby accelerating these bound electrons and rapidly causing them to become relativistic. At this stage, the electron beam is considered "stiff" and, to insure synchronization the rf phase velocity, is maintained at the speed of light throughout the remainder of the waveguide. The electrons continue to gain energy from the $\mathrm{rf}$ wave, and this is largely manifested by a continual increase in mass rather than velocity.

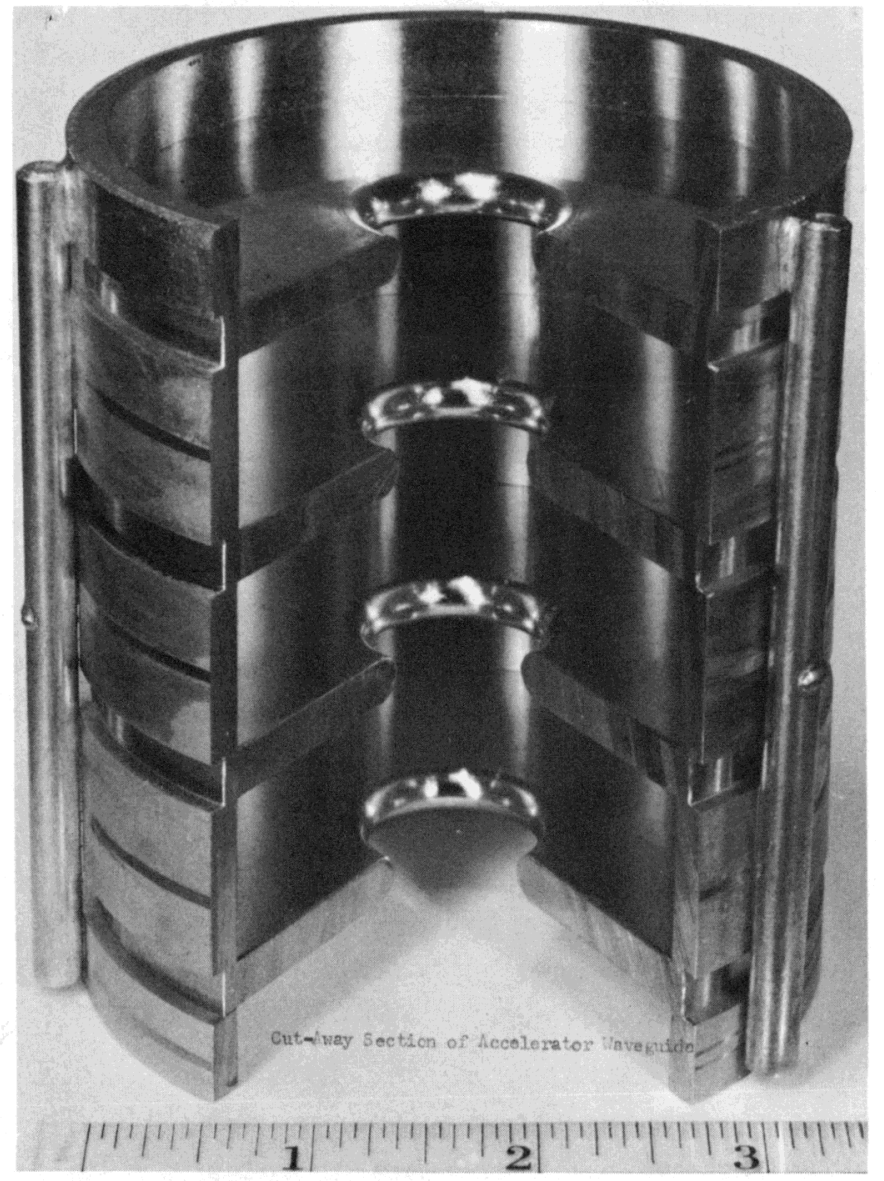

Fig. 1. Cut-away Section of Accelerator Waveguide

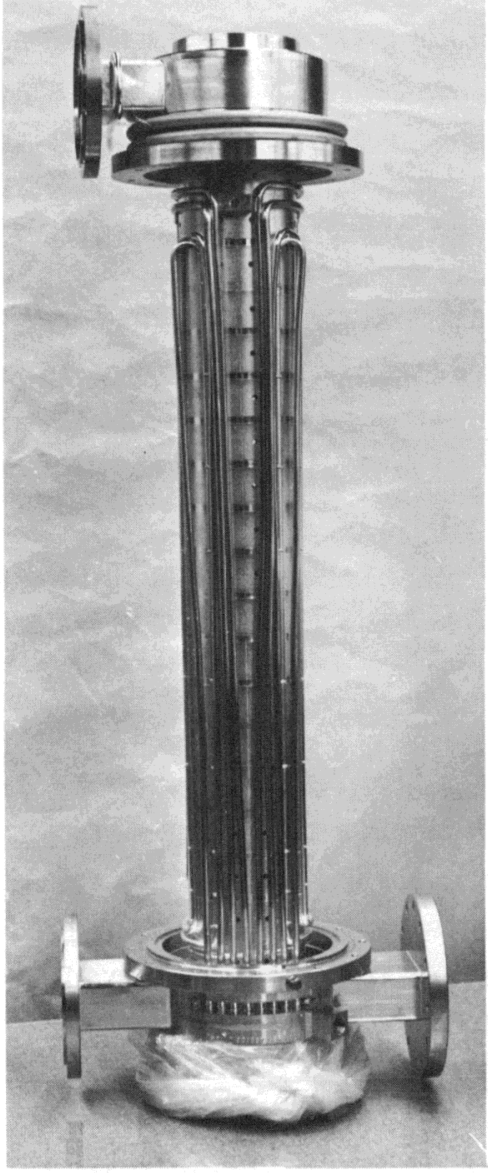

Fig. 2. Typical Waveguide Assembly Showing RF Couplers and Water Cooling

The first portion of such an accelerator waveguide is referred to as the buncher section and the remainder as the uniform section. For a given buncher configuration, the re is a definite time interval with respect to each successive cycle of the $\mathrm{rf}$ wave in which the injected electrons will be trapped and accelerated to some asymptotic phase-stable position upon the 
traveling wave. Electrons which are injected early in phase, or electrons which are too energetic or have "over-oscillated," will be in too forward a position on the wave, and they will enter an environment of continually decreasing acceleration the further they advance. These electrons will slow down and fall back with respect to the wave. Similarly, electrons that have slipped back, with respect to the synchronous phase, will experience continually increasing accelerating electric fields, and the se electrons will move forward upon the wave. This process tends to trap and then bunch the electrons at some stable position upon the rf wave (see Fig. 3).

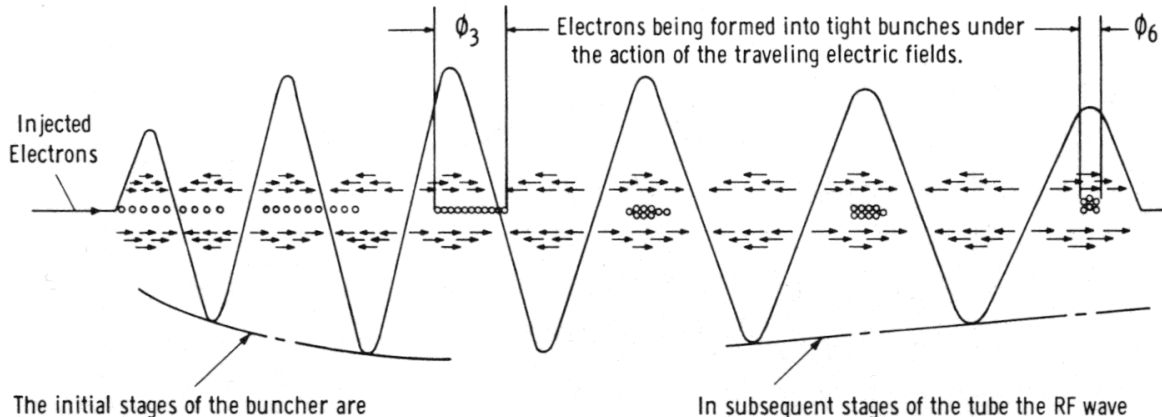

The initial stages of the buncher are arranged such that the traveling RF wave increases in amplitude, wavelength and velocity. The electron bunching process commences immediately after injection and the bunches are ultimately located at the peak of the traveling electric field.

is mainta a velocity equal to the velocity of light, the amplitude being continuously attenuated by copper losses and beam loading.

Fig. 3. Schematic Arrangement of a Traveling RF Wave and the Electron Bunching within an Accelerator Tube

Buncher designs can be arranged such that phase oscillations are controlled, and the electron bunches are ultimately located at successive peaks of the $\mathrm{rf}$ wave where they are subject to the maximum available accelerating force. Strong rf dispersion forces in the initial cavities of the buncher tend to enlarge the cross section of the electron beam. By controlling the pattern of the electron phase orbits, these, and other radial dispersion forces, can be effectively counteracted with a correctly graded axial magnetic field produced by solenoid coils and magnetic shunts, thereby resulting in a beam of minimum cross section.

Another form of waveguide design dispenses with the variable phasevelocity buncher and utilizes a uniform phase-velocity structure throughout the entire length of the accelerator. Under these circumstances, relatively large rf peak power sources are required, such that high accelerating electric fields are provided in the initial portion of the guide. Optimumvelocity electrons, which are then injected into the guide during the acceptance phase interval of each rf cycle, will become trapped, bunched, and ultimately located at the crest of the rf wave. 
As discussed in a later section, considerable improvement in accelerator performance may be gained by chopping and/or prebunching the electron beam prior to injection into the accelerator waveguide.

To obtain the highest possible energy gain in a given length of accelerator waveguide and to insure the existence of electron beams with a narrow energy spectrum, it is necessary to achieve tight electron bunches which are located at the crest of the traveling rf wave in the shortest possible distance after injection, and then maintained at this synchronous relationship throughout the length of the accelerator guide.

After acceleration, the high-energy electron beam may be extracted from the system through a thin metal window and used directly or allowed to bombard a target to produce $\mathrm{X}$ rays.

Although high-performance accelerating structures are relatively critical to manufacture, requiring internal surface finishes of a few microinches and tolerances of less than 0.0005 in., they can be relied upon in service to last indefinitely without change of characteristics.

Pulsed operation, using established techniques not unlike those developed for radar applications, allows rf peak power levels in the multimegawatt region to be obtained from relatively small components.

Usually, a modulator consisting of a high-voltage power supply, charging choke, switch tube, and pulse-forming network provides pulsed power of several microseconds duration to both the rf generator and the electron gun via a pulse transformer at a repetition rate of several hundred pulses per second.

\section{Design Considerations}

\section{Electron Energy Gain}

Under synchronous conditions, the energy imparted to an electron in a traveling wave linear accelerator may be written as

$$
V=\int_{0}^{L} E_{z} d z,
$$

where $E_{z}$ is the resultant of 2 electric field components:

(a) the value, at the position of the electron, of the longitudinal electric field, the Fourier component of which is synchronous with the electron, and 
(b) the electric field in phase and associated with the electron bunch, i.e., the beam-loading component.

This may be written as

$$
E_{z}=E_{0} e^{-I z} \cos \theta-i r\left(1-e^{-I z}\right) \text {, }
$$

where

$$
\begin{aligned}
E_{0}= & \text { the initial peak axial electric field, } \\
I= & \text { voltage attenuation per unit length of the accelerating } \\
& \text { structure, } \\
\theta= & \text { angular displacement of the electron from the position of } \\
& \text { peak accelerating force. } \\
i= & \text { peak beam current, }
\end{aligned}
$$

and

$$
\begin{aligned}
r= & \text { shunt impedance of the accelerating structure per unit } \\
& \text { length } \\
= & \text { ratio of the square of the peak voltage per unit length to the } \\
& \text { rf power dissipated per unit length. }
\end{aligned}
$$

Integrating (1), we have

$$
V=E_{0} \cos \theta K L-\operatorname{ir}(1-K) L,
$$

where

$$
K=\left(\frac{1-e^{-I L}}{I L}\right)
$$

and $L$ is the length (in meters) of the accelerator waveguide section. The voltage $\mathrm{V}$ may be expressed in terms of $\mathrm{rf}$ peak power $\mathrm{P}$ by considering the definition of shunt impedance:

$$
r=\frac{E^{2}}{-\left(\frac{d P}{d z}\right)}
$$

where

$$
P_{z}=P e^{-2 I z}
$$


Therefore,

$$
E_{0}=\sqrt{2 \operatorname{Ir} P_{0}},
$$

and substituting in Eq. (2) we get

$$
V=L\left[\begin{array}{ll}
\sqrt{2 \operatorname{Ir} P_{0}} & K \cos \theta-i r(1-K)
\end{array}\right] \quad .
$$

For zero beam current, the no-load voltage is given by

$$
\mathrm{V}_{0}=\mathrm{L} \sqrt{2 \operatorname{Ir} \mathrm{P}_{0}} \mathrm{~K}
$$

The following example will serve as an illustration. Consider a uniform accelerating waveguide in which the electron bunches are synchronous with the traveling $\mathrm{rf}$ wave and located at the peak position, i.e.,

$$
\cos \theta=1
$$

Let

$$
\begin{aligned}
\mathrm{P}_{0} & =7 \mathrm{Mw} \text { rf peak power } \\
\mathrm{I} & =0.2 \text { nepers } / \mathrm{m} \\
\mathrm{L} & =2.0 \mathrm{~m} \\
\mathrm{r} & =55 \text { megohms } / \mathrm{m} .
\end{aligned}
$$

The total voltage attenuation of the guide, IL, will therefore equal 0.4 neper and $\mathrm{K}$ will equal 0.825 . This leads to a linear relationship between voltage and peak beam current such that $\mathrm{V}=(20.48$ - i 9.63) Mev. This gives 16.6 Mev at $400 \mathrm{ma}$ of peak current, 18.5 Mev at $200 \mathrm{ma}$, or $20.48 \mathrm{Mev}$ at zero current.

A more rigorous calculation would allow for the small los in energy during the bunching process in the initial portion of the guide and for subsequent small phase drifts of the electron bunches with respect to the $\mathrm{rf}$ wave, due to accelerator guide temperature and drive frequency variations.

\section{Choice of Parameters}

Equation (3) indicates that a specific electron beam current and/or energy may be obtained with different combinations of guide length $L$, rf peak power $P$, and attenuation I. For a given guide configuration and operational frequency, the unit length shunt impedance $r$ is a slowly varying function of $I$ and may be considered constant for first-order approximations. 
Varying degrees of optimization can be applied in selecting parameter combinations so that the linac system will be especially suited to a particular application, such as to obtain maximum electron beam power or to obtain maximum X-ray output (for a given input power), to obtain maximum possible electron energy, and to obtain high stability of energy spectrum.

$\underline{\text { RF Power and Guide Length }}$

In practice, the final selection of parameters, particularly the guide length and the rf power level, is influenced by available space and operational experience and economics. Choice of the rf power source is clearly restricted to available tube types with a strong tendency toward selecting the lowest possible peak power in order to minimize power supply ratings and replacement costs. Several rf tube types and their approximate cost are shown in Fig. 4.

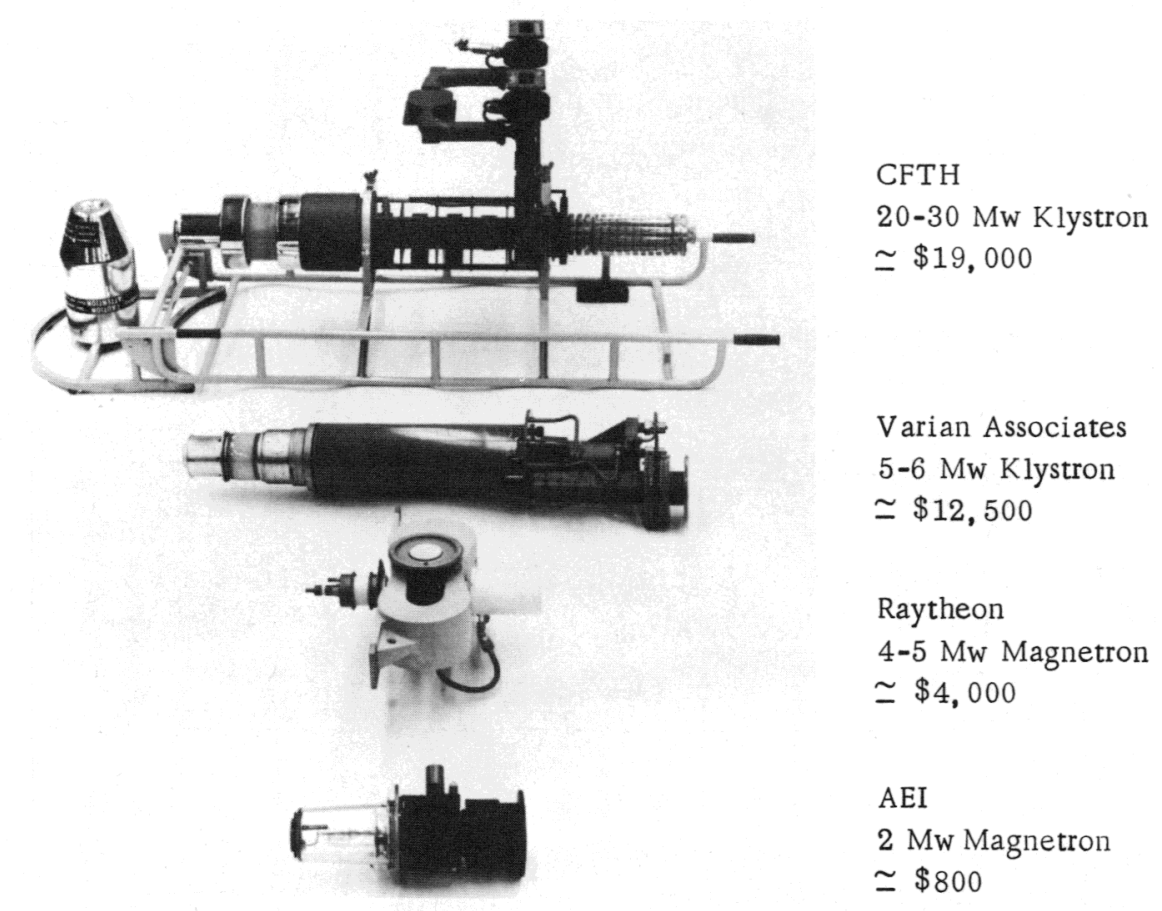

Fig. 4. Selection of Typical RF Power Tubes

Furthermore, most linac manufacturers have established circuitry and assembly layouts, and operational experience for particular rf tube types, e.g. 2, 4, 6, 12, 15 and $20 \mathrm{Mw}$, and the tendency is to select a specific rating in this range as close as possible to the desired value, and then reoptimize the remaining parameters to provide the required characteristics for the electron beam. 
In the design of maneuverable linacs for radiography and supervoltage radiotherapy, the length of the accelerator guide is usually predetermined by the requirements of the application and the geometry of the support mechanism. Generally speaking, linacs operating at $3 \mathrm{kMc}$ (' $\mathrm{S}$ ' band frequency) utilize guide section lengths which vary from 1 to $3 \mathrm{~m}$.

The attenuation per unit length of the guide is determined by the final selection of guide length and the required attenuation parameter, IL.

Attenuation Parameter IL

The attenuation parameter is defined as the overall attenuation in nepers of the accelerator guide due to $\mathrm{rf}$ dissipation in the walls, and its choice greatly influences the performance and operational characteristics of the linac.

By rearranging Eq. (3), we have

$$
V=\sqrt{\frac{2 r P_{0} L}{I L}}\left(1-e^{-I L}\right) \cos \theta-i r L\left(\frac{I L-1+e^{-I L}}{I L}\right)
$$

Maximum electron beam energy for different conditions of beam loading can be obtained from this equation by optimizing IL. For example, the noload energy will be a maximum when

$$
\left(1-e^{-I L}\right)=2 I L e^{-I L} \text {, giving } V_{0 m}=0.905 \sqrt{\operatorname{LrP}_{0}} .
$$

However, in optimizing IL to provide maximum X-ray output from a linac, consideration must be given to several additional aspects.

Dependence of RF Fill Time and Average Beam Current on IL

Because of the inductive nature of an accelerator guide, the rf requires a finite time (fill-time) to propagate through and store energy along the full length of the structure.

The propagation or group velocity $\mathrm{v}_{\mathrm{g}}$ of the rf energy is strongly dependent upon the loading of the guide (i.e., dimensions and geometry of the aperture in the discs and the thickness of the discs) and may be related to the voltage attenuation per unit length of guide I, by

$$
\mathrm{v}_{\mathrm{g}}=\pi \mathrm{f} / \mathrm{IQ} \quad \text {, }
$$

where $Q$ is defined as energy stored divided by the energy lost per radian. The fill time $T_{F}$ can now be expressed in terms of attenuation parameter:

$$
\mathrm{T}_{\mathrm{F}}=\mathrm{L} / \mathrm{v}_{\mathrm{g}}=(\mathrm{IL}) \mathrm{Q} / \pi \mathrm{f} .
$$


Current which is injected into the accelerator at the same time as the rf power is applied will not experience the full accelerating force until after one fill-time has expired. Under these circumstances, the steady-state beam current pulse length will always be less than the applied rf pulse length, and, from the above, a relationship between the attenuation parameter IL and the average electron beam current may be obtained:

$$
\begin{aligned}
\text { Average current } & =\text { Peak current } x \text { duty cycle } \\
{ }^{i_{A V}} & =i(\text { pulses per sec } x \text { current pulse length) } \\
& =i N\left(T-T_{F}\right) \\
& =i N\left(T-\frac{I L Q}{\pi f}\right),
\end{aligned}
$$

where

$$
\mathrm{N}=\mathrm{rf} \text { pulses per second }
$$

and

$$
T=\text { length of } \mathrm{rf} \text { pulses in seconds. }
$$

Furthermore, electrons which are injected into the tube during the filltime period will experience different integrated accelerating forces along the tube and will consequently emerge at a variety of energy levels extending up to the steady-state value.

The proportion of fill-time electrons may be reduced to some extent by increasing the circuit complexity and arranging to trigger the electron gun at some optimum time after the commencement of each rf pulse. However, due to the transient loading of the rf by the electron beam itself, the initial portion of each current pulse will always contain electrons of different energies from that of the steady-state value.

Dependence of X-ray Intensity on IL

In the energy range (2-40 Mev) under consideration, the X-ray intensity produced by an electron beam incident upon a thick, high-Z target may be expressed empirically as

$$
\mathrm{X}={ }^{\mathrm{ki}} \mathrm{AV} \mathrm{V}^{\mathrm{n}}
$$

where

$$
\begin{aligned}
& X=X \text {-ray intensity in rads per minute at } 1 \mathrm{~m} \\
& \mathrm{k}=\mathrm{a} \text { constant, } 0.07
\end{aligned}
$$




$$
\begin{aligned}
{ }^{i_{A V}} & =\text { Average target current in } \mu \mathrm{amp} . \\
\mathrm{V} & =\text { electron energy in } \mathrm{Mev}
\end{aligned}
$$

and

$$
\begin{aligned}
\mathrm{n}= & \text { an exponent which varies such that at } 10 \mathrm{Mev}, \mathrm{n} \approx 2.7, \\
& \text { and at } 25 \mathrm{Mev}, \mathrm{n} \approx 2.5 .
\end{aligned}
$$

The optimum values of electron energy, peak current, and attenuation parameter to produce maximum X-ray output can now be obtained by partial differentiation of Eq. (9) after substitution from Eqs. (3) and (8):

$$
\begin{aligned}
& X=k i N\left(T-\frac{I L Q}{\pi f}\right)\left[\begin{array}{ll}
\sqrt{2 \operatorname{Ir} P_{0}} & K L \cos \theta-i r(1-K) L
\end{array}\right]^{n} \\
& \frac{\partial X}{\partial i}=k N\left(T-\frac{I L Q}{\pi f}\right) V^{n-1}[V+\operatorname{nrLi}(K-1)]
\end{aligned}
$$

When $\partial x / \partial i=0$, we have

$$
i_{x m}=\sqrt{\frac{2 I P_{0}}{r}}\left(\frac{K}{1-K}\right) \frac{\cos \theta}{n+1}
$$

and

$$
\mathrm{V}_{\mathrm{xm}}=\mathrm{L} \sqrt{2 \mathrm{Ir} \mathrm{P}_{0}}\left(\frac{\mathrm{n}}{\mathrm{n}+\mathrm{l}}\right) \mathrm{K} \cos \theta \quad .
$$

Also, from Eq. (10),

$$
\begin{aligned}
\frac{\partial \mathrm{X}}{\partial(\mathrm{IL})}= & \mathrm{kiNV}^{\mathrm{n}-1}\left[\mathrm{~nL}\left(\mathrm{~T}-\frac{\mathrm{ILQ}}{\pi f}\right)-\frac{\mathrm{VQ}}{\pi f}\right]\left\{\left[\sqrt{\frac{2 r \mathrm{P}_{0}}{\mathrm{~L}}} \sqrt{\mathrm{IL}} \cos \theta+\mathrm{ri}\right] \frac{\partial \mathrm{K}}{\partial \mathrm{IL}}\right. \\
& \left.+\frac{1}{2} \sqrt{\frac{2 r \mathrm{P}_{0}}{\mathrm{~L}}} \frac{\mathrm{K} \cos \theta}{\sqrt{\mathrm{IL}}}\right\} .
\end{aligned}
$$

Substituting Eqs. (11) and (12), for $\partial \mathrm{x} / \partial \mathrm{IL}=0$ we have

$$
-\frac{K Q}{\pi f(n+1)}+\left(T-\frac{I L Q}{\pi f}\right)\left[\left(1+\frac{K}{(1-K)(n+1)}\right)\left(\frac{1-(1+I L) K}{I L}\right)+\frac{K}{2 I L}\right]=0
$$

where

$$
\frac{\partial \mathrm{K}}{\partial \mathrm{IL}}=\frac{1-(1+\mathrm{IL}) \mathrm{K}}{\mathrm{IL}}
$$


by making

$$
A=T \pi f / Q \quad \text {. }
$$

Thus

$$
\begin{gathered}
K^{2}\left[I L+(A-I L)\left(n I L+\frac{n}{2}-\frac{1}{2}\right)\right]+K[-I L-(A-I L) \\
\left.\left\{I L(n+1)+\frac{3 n}{2}+\frac{1}{2}\right\}\right]+(n+1)(A-I L)=0 .
\end{gathered}
$$

Equation (15) provides the optimum value of IL, and Eqs. (11) and (12) establish the values of peak beam current, $i_{x m}$, and electron energy, $\mathrm{V}_{\mathrm{xm}}$, to produce maximum $\mathrm{X}$-ray intensity. These quantities may be expressed more conveniently in terms of the zero-current energy $V_{0}$ by combining Eqs. (4) and (12):

$$
V_{\mathrm{xm}}=V_{0}\left(\frac{n}{n+1}\right)=V_{0}-i_{x m} r L(1-K)
$$

Therefore

$$
i_{\mathrm{xm}}=\mathrm{V}_{0} / \mathrm{rL}(1-\mathrm{K})(1+\mathrm{n}) \quad .
$$

As an example, we can solve for optimum IL from Eq. (15) for a 25-Mev linac operating at ' $S$ ' band frequency. Under these circumstances,

$$
\mathrm{n} \approx 2.5
$$

and from Eq. (14),

$$
\begin{aligned}
A & =T \times 6.9 \times 10^{5} \\
& =2.42(\text { for, say, } 3.5 \mu \text { sec pulses })
\end{aligned}
$$

This gives an optimum value for IL of $0.413(\mathrm{~K}=0.821)$. From (4) and (16) we have

$$
\begin{aligned}
\mathrm{V}_{0} & =25\left(\frac{3.5}{2.5}\right)=35 \mathrm{Mev} \\
& =\sqrt{2(\mathrm{IL}) \mathrm{rLP} \mathrm{K}} \\
& =5.53 \sqrt{\mathrm{LP}_{0}},
\end{aligned}
$$

which uniquely defines the product of $L$ and $P_{0}$ as 40 for the above conditions using a single section of accelerator guide, or of 10 if 2 accelerator guide sections with the same value of IL are used and separately fed with rf power. 
X-ray intensity for varying values of electron beam power can be calculated directly from Eq. (10), and final allowances should then be made for absorption and collimation losses. Figure 5 shows typical electron beam and X-ray output characteristics for a high-energy linac.

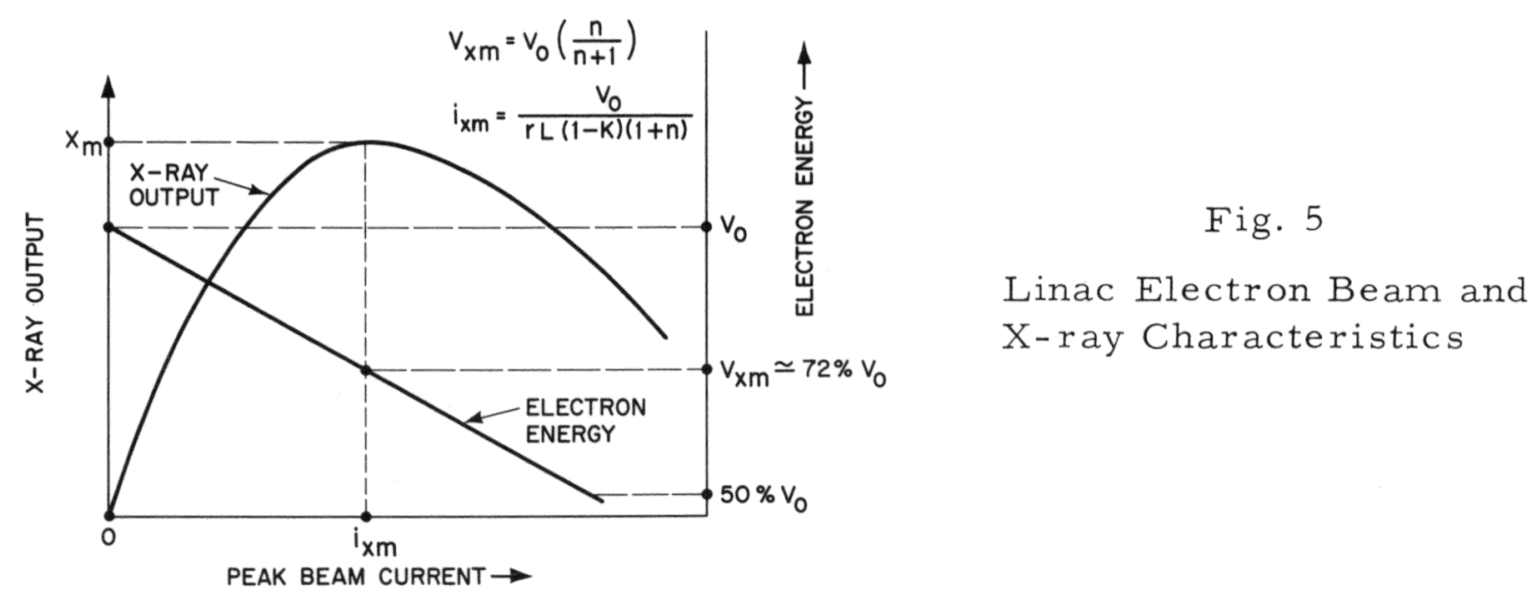

Dependence of X-ray Output Stability on IL

Another aspect which should be considered when choosing a value of IL is the degree of energy variation of electrons due to small fluctuations in frequency from the rf generator.

The guide attenuation is related to the group velocity as previously shown in Eq. (6). However, the group velocity is also related to the degree of departure of the accelerator waveguide wavelength from its design value for a given change in drive frequency.

The dispersive characteristic of an accelerator guide is such that small changes in drive frequency can cause relatively large changes in the waveguide wavelength, with the resultant loss of phase relationship between cavities. When this occurs, the phase velocity of the traveling rf wave no longer remains synchronous with the electron bunches. Electrons, normally positioned at the peak of the rf field, will be subjected to weaker accelerating forces, causing a reduction in electron energy and an even larger reduction in X-ray output [due to the exponent ' $n$ ' in Eq. (9)].

In order to evaluate this effect, the definition of group velocity can be used to obtain an expression of electron phase and energy variation for a given change in drive frequency:

$$
v_{g}=c \frac{d\left(\frac{1}{\lambda_{0}}\right)}{d\left(\frac{1}{\lambda_{g}}\right)}
$$


where

$$
\begin{aligned}
c & =\text { velocity of light } \\
\lambda_{0} & =\text { free space wavelength }(=\mathrm{c} / \mathrm{f}) \\
\lambda_{\mathrm{g}} & =\text { accelerator waveguide wavelength }
\end{aligned}
$$

and, at a phase velocity equal to that of light, this reduces to

$$
\left(\frac{\Delta \lambda_{g}}{\lambda_{g}}\right)=-\frac{c}{v_{g}}\left(\frac{\Delta f}{f}\right)
$$

The change in phase of the rf wave measured at the end of the accelerator guide for a change $\Delta \mathrm{f}$ in operational frequency will therefore be

$$
\begin{aligned}
\Delta \theta & =-\left(\frac{\Delta \lambda_{g}}{\lambda_{g}}\right) \frac{L}{\lambda_{g}} 2 \pi \\
& =\frac{c}{v_{g}}\left(\frac{\Delta f}{f}\right) \frac{L}{\lambda_{g}} 2 \pi,
\end{aligned}
$$

and, substituting from Eq. (6),

$$
\Delta \theta=\left(\frac{\Delta f}{f}\right) 2 Q(\text { IL) } .
$$

By making $\theta$ a function of $\mathrm{z}$ and integrating Eq. (1), it can be shown that the change in zero-current electron energy is

$$
\left(\frac{\Delta \mathrm{V}_{0}}{\mathrm{~V}_{0}}\right)=\left[\left\{\frac{\left[1-\left(\cos \theta_{L}-\frac{\theta_{L}}{\mathrm{IL}} \sin \theta_{\mathrm{L}}\right) \mathrm{e}^{-\mathrm{IL}}\right]}{\mathrm{IL}\left[1+\left(\frac{\theta_{\mathrm{L}}}{\mathrm{IL}}\right)^{2}\right]\left[\frac{1-\mathrm{e}^{-I L}}{\mathrm{IL}}\right]}\right\}-1\right]
$$

where $\theta_{L}$ is the angle through which an electron, initially at the peak of the rf wave, has slipped with respect to the wave by the time it reaches the end of the accelerator guide. In other words, $\theta_{\mathrm{L}}$ is given by Eq. (18).

This variation in energy due to a given change in operational frequency will increase with beam loading and when oper ating under optimum $\mathrm{X}$-ray output conditions:

$$
\begin{aligned}
\left(\frac{\Delta \mathrm{V}_{\mathrm{xm}}}{\mathrm{V}_{\mathrm{xm}}}\right) & =\left(\frac{\mathrm{n}+\mathrm{l}}{\mathrm{n}}\right)\left(\frac{\Delta \mathrm{V}_{0}}{\mathrm{~V}_{0}}\right) \\
& =\left(\frac{\mathrm{n}+\mathrm{l}}{\mathrm{n}}\right)[\mathrm{Eq} . \text { (19)]. }
\end{aligned}
$$


An example will serve to show the order of X-ray intensity variation that will occur for a given change in operational frequency. Using the example quoted on page 223, and for electron bunches initially at the crest of the $\mathrm{rf}$ wave, a $100-\mathrm{kc}$ change in frequency at $3 \mathrm{kMc}$ with a guide $Q$ of 13000 would result in

$$
\theta_{L}=21^{\circ} \quad[\text { from Eq. (18) }]
$$

giving

$$
\left(\frac{\Delta \mathrm{V}_{\mathrm{xm}}}{\mathrm{V}_{\mathrm{xm}}}\right)=-2.8 \%
$$

and

$$
\left(\frac{\Delta \mathrm{X}_{\mathrm{m}}}{\mathrm{X}_{\mathrm{m}}}\right)=-7.4 \%
$$

For a 200-kc variation, however,

$$
\begin{aligned}
\theta_{\mathrm{L}} & =42^{\circ} \\
\left(\frac{\Delta \mathrm{V}_{\mathrm{xm}}}{\mathrm{V}_{\mathrm{xm}}}\right) & =-10.4 \%
\end{aligned}
$$

and

$$
\frac{\Delta \mathrm{X}_{\mathrm{m}}}{\mathrm{X}_{\mathrm{m}}}=-25.7 \%
$$

This reduction may be manifest by either a fluctuation in X-ray output or a reduced mean value depending on the nature of the frequency drift.

From the foregoing it becomes clear that, in choosing a value of IL, consideration must also be given to the frequency fluctuations, during the pulse or from pulse to pulse, that can be expected from the chosen combination of modulator, $r f$ tube, and waveguide system.

By suitable compromise of parameters and the use of microwave techniques to control variable impedances that may be presented to the rf tube, highly stable X-ray outputs may be achieved from linacs using relatively unstable frequency sources.

By using similar arguments to the above, the dependence of X-ray output stability on choice of IL can also be related to cavity phase shifts caused by thermal variations along the guide. Under normal circumstances, however, this is avoided by maintaining the guide temperature to within $1{ }^{\circ} \mathrm{C}$ or less. 
$\underline{\text { Post-acceleration Beam Bending }}$

Stability of the electron beam energy, combined with a narrow energy spectrum and a small beam cross section, assists in reducing spectroscopic divergence during post-acceleration bending of the beam. This technique, which may also utilize energy focus concepts in the bendingmagnet system, can provide a small, very stable X-ray focal source with excellent radiographic qualities. (6) The X-ray beam can be projected at right angles to the accelerator waveguide by arranging for the bentelectron beam to be focused onto a transmission-type target. Furthermore, use of a rotating $\mathrm{X}$-ray head permits the $\mathrm{X}$-ray beam to be projected through $360^{\circ}$ in a vertical plane while the accelerator waveguide lies in a horizontal position, thereby providing a maneuverable linac system of greater flexibility (see Figs. 6 and 7).

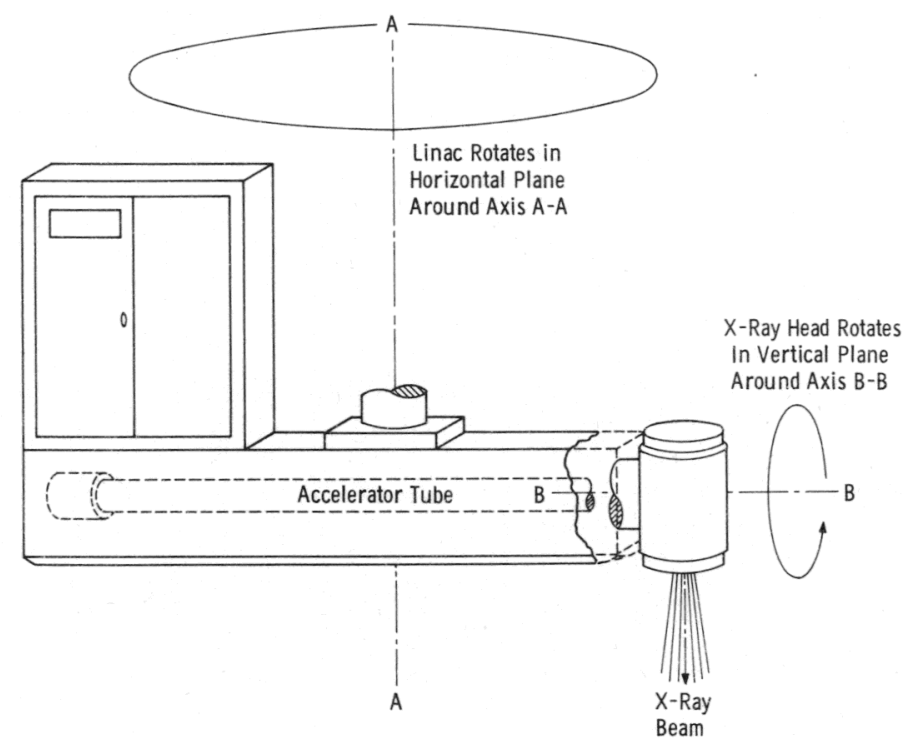

Fig. 6

Rotational Movements of Radiographic Linac

The advantages offered by such systems (7) over other types of industrial X-ray generators has led to the development of a range of linacs for high-energy X-ray production, with outputs of up to 40,000 rads/min at a meter, and electron energies up to $30 \mathrm{Mev}$.

Control and stability of the high-energy electron trajectories during beam bending and immediately prior to striking the target is of the utmost importance in radiographic and, particularly, radiotherapy applications in which it is necessary that the spatial polar intensity distribution of the Xray beam remains constant and preferably symmetrical with respect to the beam-defining collimation system.

Critical operational settings may be avoided and satisfactory X-ray performance assured if the linac produces a stable, narrow-energy-spread 
electron beam of small cross section. Also, by combining the se features with beam-bending techniques as described above, loss of beam current due to collimation and spectroscopic divergence can be considerably reduced, resulting in higher X-ray output and less leakage radiation from the machine.

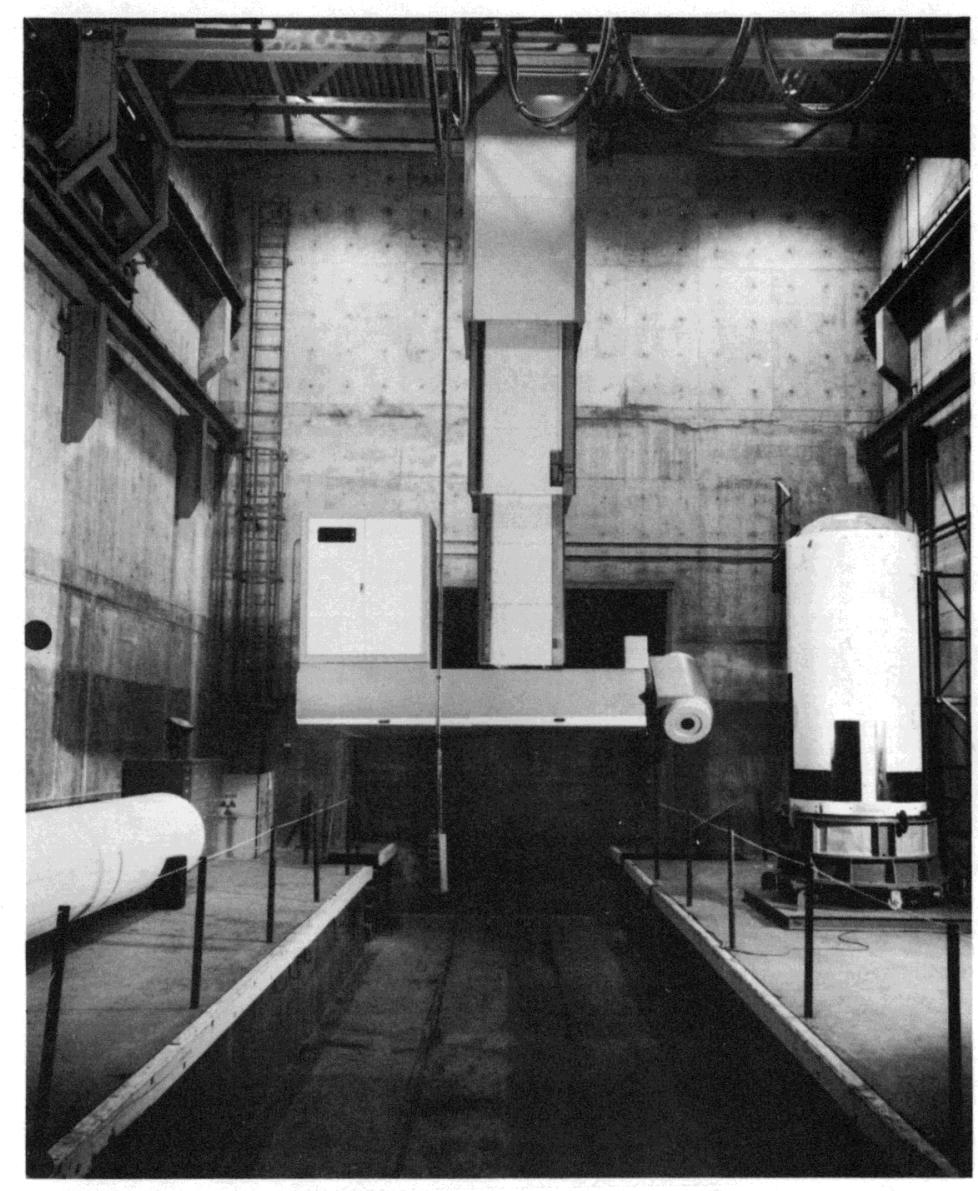

Fig. 7. 10-Mev Radiographic Linac Installation at NAD Concord, Showing Telescopic Hoist and Bridge Crane Suspension. A First Stage Polaris Motor is Shown at Right.

It is beyond the scope of this paper to analyze the linac beam optics in detail; however, several aspects relating directly to the achievement of sharp energy spectrums and small cross sections will be briefly discussed.

\section{Linac Electron Beam Optics}

High-energy, sharp-spectrum beams are produced with linacs by subjecting electrons to a succession of varying electromagnetic environments. 
The various stages of interaction encountered by the electrons in their trajectory throughout the linac are outlined below:

(a) Source of electrons and initial beam shaping.

(b) Beam preparation prior to injection into the accelerator guide (chopping, pre-bunching, focusing, steering, scanning, etc.).

(c) Electron bunching, acceleration, and bunch positioning with respect to the $\mathrm{rf}$ wave.

(d) Main acceleration.

(e) Post-acceleration application requirements (i.e., bending, focusing, scanning, splitting, etc。).

Electron Source and Beam Shaping

Several reliable designs of electron guns are available for linac operation. These guns produce up to several amps of beam current in the voltage range from 50 to $150 \mathrm{kv}$, and are normally operated under pulsed conditions such that high voltage is applied to the cathode for several microseconds at a pulse repetition frequency, which may be varied to suit operational conditions, of up to 1000 pulses per second or more. By employing a pure metal emitter, the danger of cathode poisoning is minimized should the vacuum system be inadvertently opened to atmosphere during operation.

The use of indirect heating of the cathode button avoids distortions of the electron trajectory due to the magnet fields associated with the heater current, and also permits electron bombardment operation.

With this technique, a potential of several thousand volts is applied between the cathode and the heater filament, thereby driving electrons into the button and raising it to emission temperature at a.considerably reduced level of filament power.

The bomber gun has been used extensively in high-power klystrons, has a long cathode life, and, by using a feedback control sensitive to bombarder current, the filament power may be automatically regulated to ensure constant electron emission.

Guns of relatively low perveance* and Pierce-type geometry are commonly used in linacs. This type of gun may be operated under spacecharge or temperature-limited conditions and makes use of carefully shaped electrodes to provide a convergent, axially symmetric electron beam.

*e.g., perveance of $0.1 \times 10^{-6}$, where perveance $=$ Beam Current $/(\text { Gun Voltage })^{3 / 2}$. 
Beam Preparation Prior to Injection into the Accelerator Guide

Advantage may be taken in the region between the gun and the accelerator waveguide input to prebunch the dc electron beam and arrange for the bunches to enter the accelerator at the most acceptable phase with respect to each cycle of the traveling $\mathrm{rf}$ wave. The final section of this paper deals more specifically with some of the advantages to be gained by this technique.

A variety of methods are available for producing bunched electron beams suitable for injection into an accelerator guide.

One method makes use of a cavity resonant in the $\mathrm{TM}_{010}$ mode and arranged such that a longitudinal electric field exists across a gap within the cavity. A dc electron beam injected through the cavity, parallel to the electric field lines, will become velocity modulated due to the cyclic amplitude variation of the $\mathrm{rf}$ voltage across the gap in much the same manner as in a klystron amplifier tube.(8)

Because of the time-variable field interaction, electrons will emerge from the prebuncher cavity at different velocities, and cause the beam to exhibit varying levels of charge density at different distances along the drift space such that a longitudinal bunching action is set up.

The rf peak power necessary to develop a suitable gap voltage is quite low and is usually coupled from the main linac drive system through a phase shifter.

For a given ratio of peak rf gap voltage to gun injection voltage, there will be an optimum drift space length, at the end of which (see X-X Fig. 8) "ideal" bunching will occur.

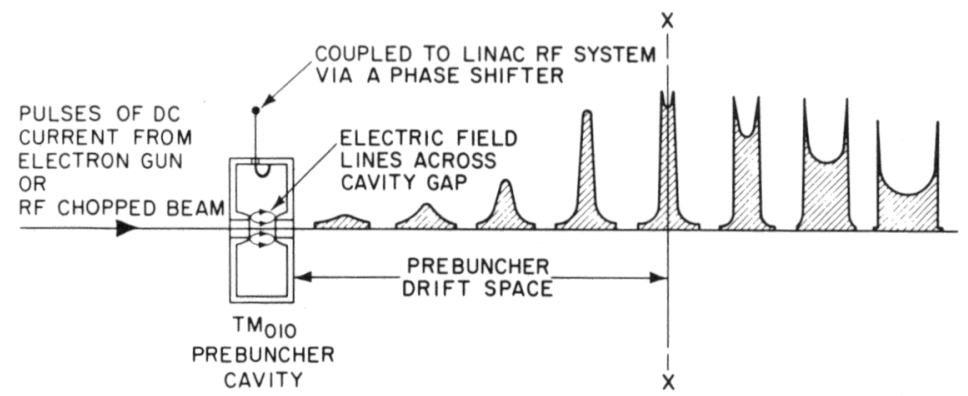

Fig. 8. Schematic Arrangement Showing Electron Bunching Action in Drift Space Due to RF Velocity Modulation.

Some applications require adjustable bunching characteristics in order to obtain optimum performance. This may be provided by maintaining 
the length of drift space between the prebuncher and the accelerator input plane $(\mathrm{X}-\mathrm{X})$ constant, and varying the power and phase of the prebuncher cavity drive.

Figure 9 shows a typical prebuncher cavity and drift space assembly during manufacture.

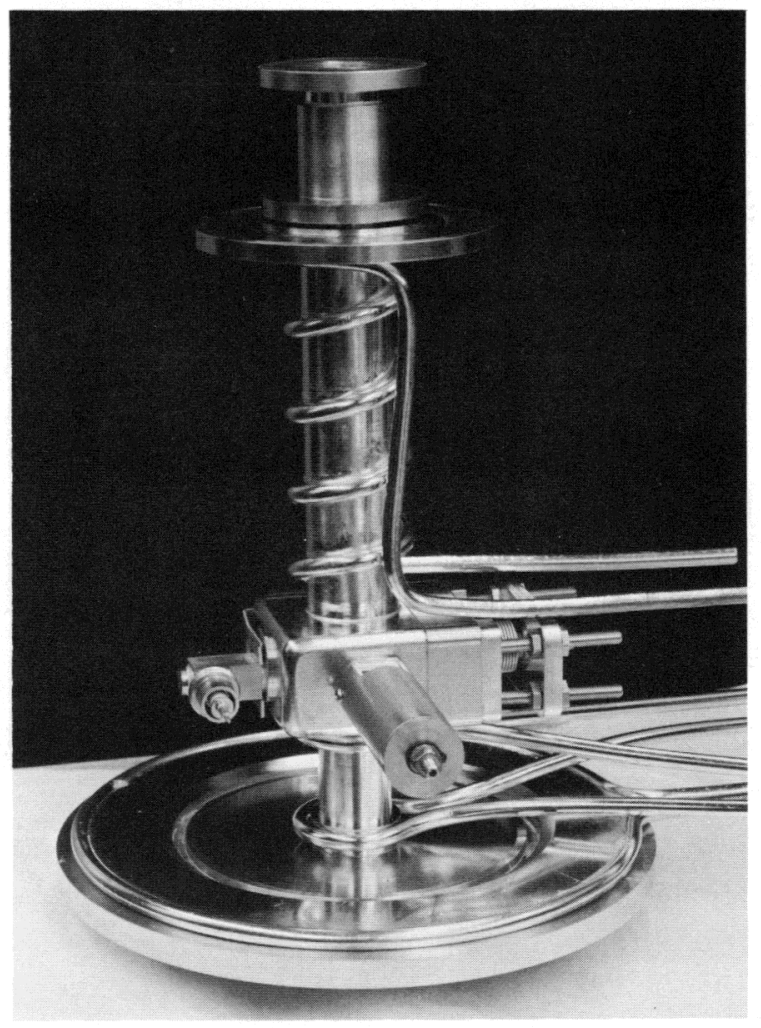

\section{Fig. 9}

Typical Prebuncher Assembly

During Manufacture

\section{Prebuncher Electron Optics}

By means of some simple kinematic theory, the prebunched electron beam may be analysed and some design parameters established. Let

$t_{1}=$ time of departure of an electron from the prebuncher,

$t_{2}=$ time of arrival at the end of the drift space,

and

$S=$ length of drift space.

Then

$$
t_{2}=t_{1}+\frac{S}{v_{m}},
$$


where

$$
\mathrm{v}_{\mathrm{m}}=\text { velocity of the modulated electron. }
$$

Also, let

$$
v_{0}=\text { electron speed corresponding to the gun voltage } V_{0}
$$

and

$$
\begin{aligned}
& \mathrm{T}_{0}=\text { time for an electron of this velocity to traverse the drift } \\
& \text { space. }
\end{aligned}
$$

Then

$$
\mathrm{v}_{0} \mathrm{~T}_{0}=\mathrm{v}_{\mathrm{m}} \mathrm{T}_{\mathrm{m}}
$$

and

$$
\mathrm{t}_{2}=\mathrm{t}_{1}+\mathrm{T}_{0}\left(\frac{\mathrm{v}_{0}}{\mathrm{v}_{\mathrm{m}}}\right),
$$

or, in terms of phase, we have

$$
\begin{aligned}
\omega t_{2}-\omega T_{0} & =\omega t_{1}+\omega T_{0}\left(\frac{v_{0}}{v_{m}}-1\right) \\
\phi_{2}-\phi_{0} & =\phi_{1}+\phi_{0}\left(\frac{v_{0}}{v_{m}}-1\right)
\end{aligned} .
$$

Here $\phi_{0}$ is the rf phase shift corresponding to the time required for an unmodulated electron to traverse the drift space. This may be expressed in terms of the free space wave length, $\lambda_{0}$, such that

$$
\phi_{0}=2 \pi \frac{S}{\beta_{0} \lambda_{0}} \quad\left(\beta_{0}=v_{0} / c\right),
$$

giving

$$
\phi_{2}-\phi_{0}=\phi_{1}+\frac{2 \pi S}{\beta_{0} \lambda_{0}}\left(\frac{\beta_{0}}{\beta_{\mathrm{m}}}-1\right),
$$

where

$$
\beta_{\mathrm{m}}=\mathrm{v}_{\mathrm{m}} / \mathrm{c} .
$$


Relativistic corrections can be considered by making

$$
\beta_{m}=\sqrt{\frac{\gamma_{m}^{2}-1}{\gamma_{m}^{2}}}
$$

where

$$
\gamma_{\mathrm{m}}=\left(\frac{\mathrm{v}_{\mathrm{m}}}{51 \mathrm{l}}+1\right)
$$

and

$$
\mathrm{v}_{\mathrm{m}}=\mathrm{V}_{0}+\mathrm{v}_{\mathrm{g}} \mathrm{B} \sin \phi_{1}
$$

where

$\mathrm{V}_{0}=$ gun voltage in kev.

$\mathrm{V}_{\mathrm{g}}=$ peak effective cavity gap voltage in kev.

$\mathrm{B}=$ a correction for transit time. This allows for the change in modulating field during the actual transit of the electron across the gap.

Figure 10 shows curves of the Eq. (21) relationship between the departure phase, $\phi_{1}$, and normalized arrival phase, $\phi_{2}-\phi_{0}$, for 2 values of $\mathrm{R}$, where in this presentation

$$
\mathrm{R}=\pi \mathrm{SV} g / \beta_{0} \lambda_{0} \mathrm{~V}_{0}
$$

The diagonal line represents the relationship for unmodulated electrons, and the departure from this is an indication of the degree of interaction between the electron and the prebuncher field.

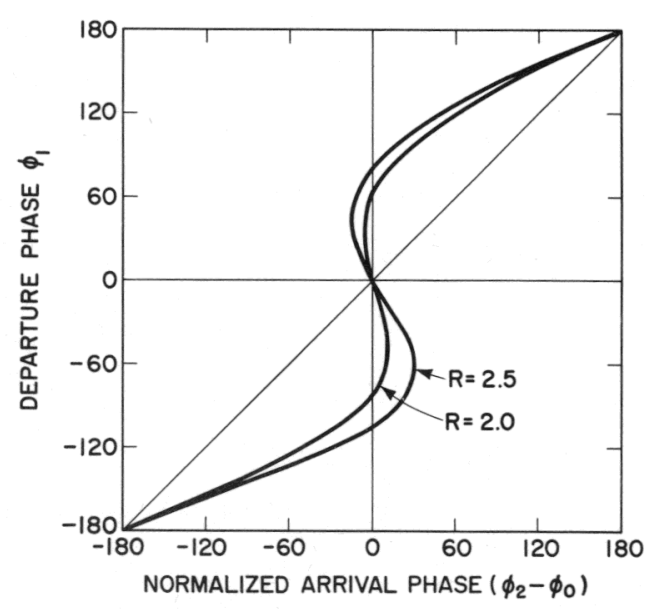

Fig. 10

Departure-Arrival Phase Relationship for Velocity Modulated Electron Beam 
It can be noted that for positive departure phases, the electrons have been increased in velocity, so that they arrive early in phase. The opposite applies for negative departure phases. This results in a bunching action, as indicated by the "compression" of the arrival phase values. The asymmetry is due to the difference in relativistic correction.

In order to determine the distribution of current with respect to the arrival phase, we have, in accordance with the law of conservation of charge,

$$
I_{2}=I_{0} \frac{d t_{1}}{d t_{2}} \quad,
$$

where

$$
\begin{aligned}
\mathrm{I}_{0}= & \mathrm{dc} \text { beam current } \\
\mathrm{dt}_{1}= & \text { a small time interval at departure from the prebuncher } \\
\mathrm{dt}_{2}= & \text { time interval (may be larger or smaller than } \mathrm{dt}_{1} \text { ) at the end } \\
& \text { of the drift space which the charge } \mathrm{I}_{0} \mathrm{dt}_{1} \text { takes to pass } \\
\mathrm{I}_{2}= & \text { the instantaneous current during the time interval } \mathrm{dt}_{2},
\end{aligned}
$$

or

$$
I_{2}=I_{0} \frac{\mathrm{d} \phi_{1}}{\mathrm{~d} \phi_{2}}
$$

By plotting curves of $\mathrm{d} \phi_{1} /\left[\mathrm{d}\left(\phi_{2}-\phi_{0}\right)\right]$, the characteristics of the bunched current are obtained, as shown in Fig. 11. It can be noted, for example, that when $R=2.5,62$ per cent of the dc current is compressed into a $46^{\circ}$ bunch compared with 12.8 per cent which would have arrived in the same phase interval had the prebuncher not been used.

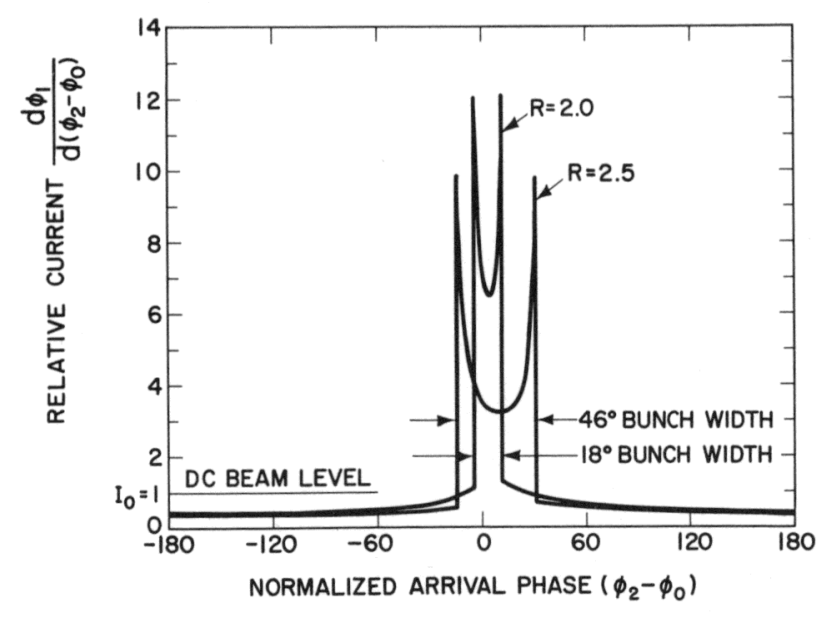

Fig. 11

Electron Density - Arrival Phase Relationship, for Velocity Modulated Beam 
It can also be noted that the remainder of the current is spread out over the rest of the cycle. This unwanted current may be avoided by using a simple rf chopper technique which prevents half of each cycle from entering the prebuncher.

Under the se circumstances and for $\mathrm{R}=2.0$ (see Fig. 12), a plus and minus $90^{\circ}$ departure phase results in all the current being bunched down to $30^{\circ}$ prior to entering the accelerator. This insures complete elimination of the unwanted current while still maintaining a good collection ratio, i.e., 50 per cent of the dc current.

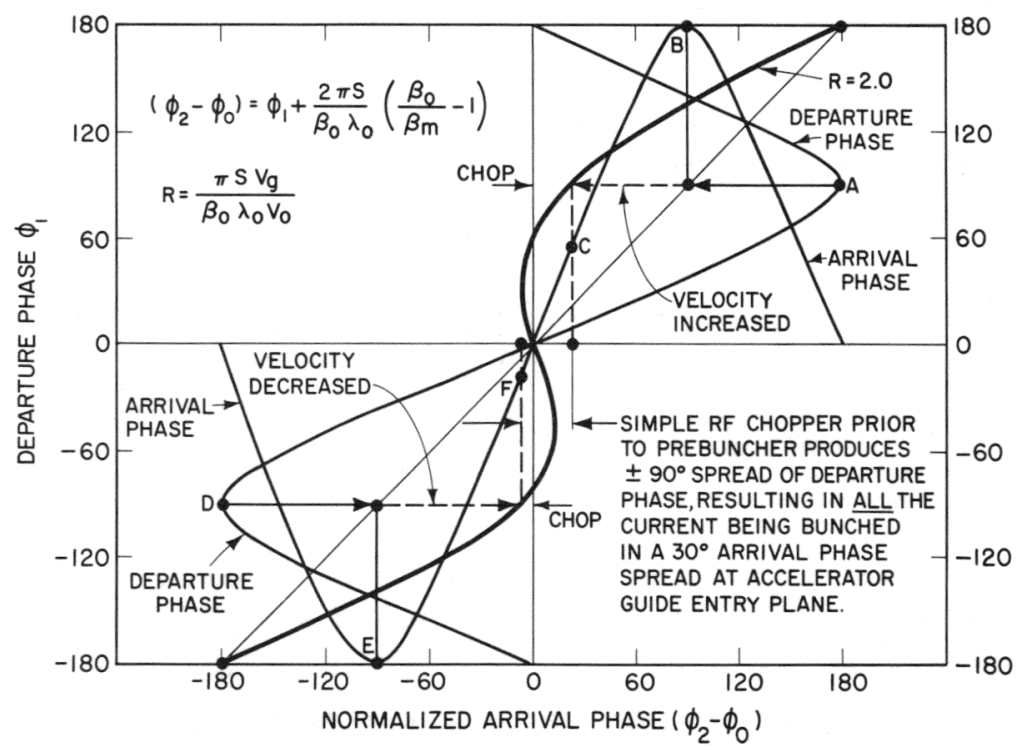

Fig. 12. Departure-Arrival Phase Curve Showing Position of Electron With Respect to the RF Wave and Effect of Using a Chopper Prebuncher Combination

For injection currents of high density, corrections must be made to allow for longitudinal debunching due to space-charge forces, and the prebuncher cavity must be capable of large signal modulation.

Uniformity of the pulse voltage of the gun cathode is particularly important for the successful operation of a prebuncher. Small values of gun-voltage ripple can result in a considerable broadening of the prebunched beam because of phase modulation, e.g., at $3 \mathrm{kMc}$ with a $100-\mathrm{kv}$ gun and a drift space of $30 \mathrm{~cm}$, the bunch width increases $7.2^{\circ}$ per 1 per cent of gunvoltage ripple.

Facility is also usually provided in the drift space to focus and steer accurately the prebunched electron beam into the accelerator guide. 
Electron Bunching Within the Accelerating Waveguide

The electron sorting and bunching processes which occur in the initial section of an accelerator waveguide can be considered for the 2 different conditions mentioned in the introduction to this paper. The first, in which the phase velocity of the guide is constant at the velocity of light, and the second, in which a buncher is employed at the beginning of the guide such that the phase velocity more nearly matches that of the injected electrons and is then arranged to increase up to the speed of light. In the former case, high injection velocity and/or high accelerating fields are necessary to insure electron capture. The acceptance phase angle over which this capture can occur is typically of the order of $180^{\circ}$.

Figures 13 and 14 show the phase-orbit plots and rate of gain of energy as electrons proceed along a uniform velocity of light guide for an injection voltage of $150 \mathrm{kv}$ and an initial field strength of $140 \mathrm{kv} / \mathrm{cm}$.

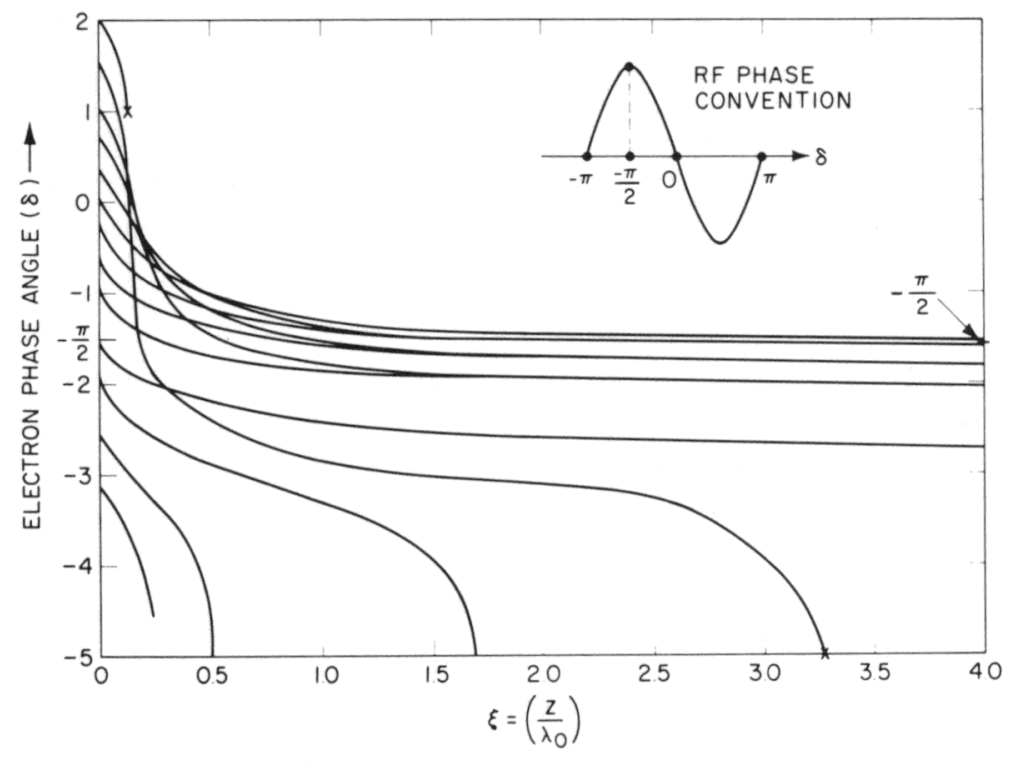

Fig. 13. Phase Orbits of Electrons Injected into a Uniform Velocity of Light Accelerator Waveguide

It should be noted that for dc injection approximately 48 per cent of the current is captured, that is, bound to accelerating orbits, and most of the remainder is rejected within the initial cavities.

Furthermore, 23 per cent of the bound electrons (11 per cent of the dc current) is injected within the range $\delta_{0}= \pm 20^{\circ}$, and this is ultimately phase compressed to produce bunches approximately $3^{\circ}$ wide, which are asymptotically located at the peak of the traveling wave, thereby resulting in a sharp energy spectrum of maximum peak value. 


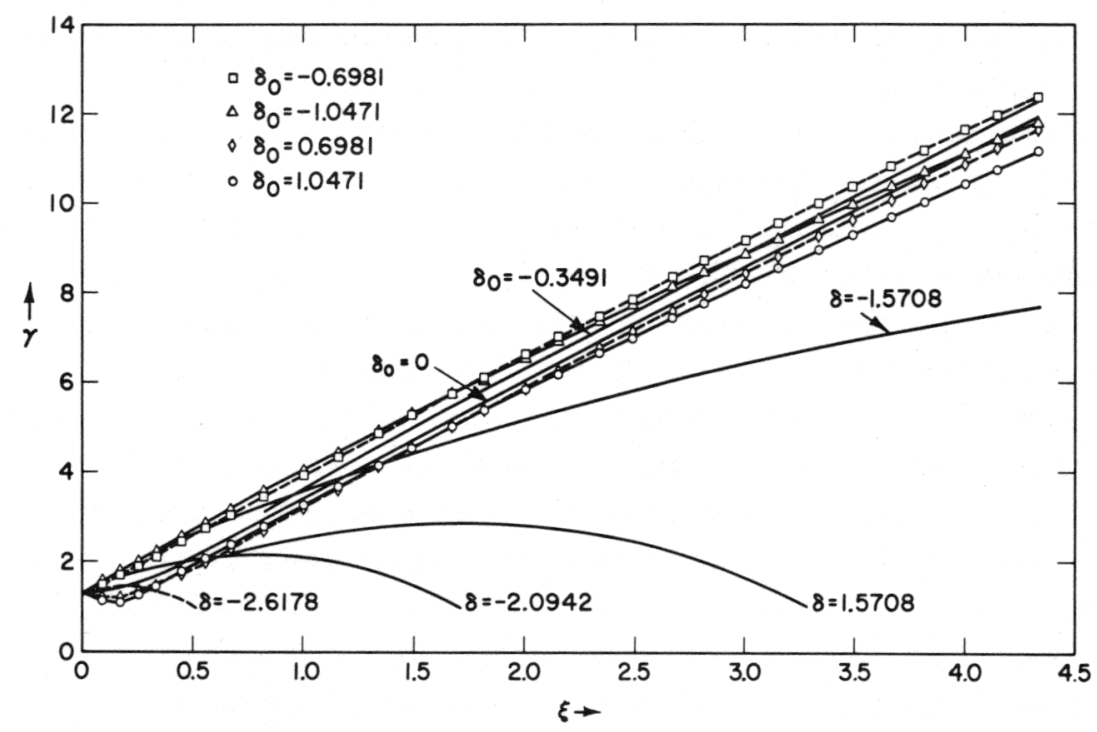

Fig. 14. Electron Energy Variations Corresponding to Fig. 13 Phase Orbits

From the point of view of achieving an extremely narrow energy spread, most of the remaining 77 per cent of accepted current (37 per cent of the dc beam) is undesirable, because the bound electrons travel through the accelerator structure at various phase positions with respect to the peak of the rf wave.

By prebunching the beam prior to injection, it can be shown by considerations similar to Fig. 11 that, for the above case, approximately 80 per cent of the dc beam will fall within the acceptance range of the accelerator structure compared with the previous figure of 48 per cent.

Furthermore, by suitable adjustment of the prebuncher phase shifter, 60 per cent of the dc current can be injected within the range $\delta_{0}= \pm 20^{\circ}$ compared with 11 per cent previously, and the "undesirable" current will be reduced from 37 per cent to 20 per cent of the dc level.

Under these circumstances, the use of a prebuncher results in an increase by a factor of 10 in the ratio of "desirable" to "undesirable" current.

A further improvement can be gained by utilizing a chopperprebuncher (see Fig. 12), in which case all of the accepted current (50 per cent of the dc beam) can be injected within the phase range $\delta_{0}= \pm 20^{\circ}$ and the undesirable current is completely eliminated.

Velocity (and phase) modulation and injection over even a small range of phase angles cause different amounts of energy to be gained in the different orbits, particularly over the first 2 wavelengths of accelerator structure. 
From an analysis of the se energy variations, optimum injection conditions may be ascertained, such as under or over bunching, entry phase, etc., in order to provide maximum output current with a minimum spread of energy.

When lower peak power rf sources and lower gun voltages are utilized, it is usually necessary to provide a variable phase velocity buncher prior to the uniform (velocity of light) section of the accelerator waveguide to insure adequate electron capture and subsequent bunching. In this case the dimensions of the cavities are varied to progressively alter the attenuation and shunt impedance along the guide in accordance with the desired laws of electric field strength as well as phase velocity (refer to Fig. 3). Suitable combination of the laws of variation of the electric field strength and phase velocity along the buncher provides control over the capture efficiency, bunching efficiency, and energy spread of the electron beam prior to entering the uniform section of the guide.

In some cases transition sections are necessary between the buncher and the uniform accelerator guide to provide a phase shift and/or prevent rf reflections.

Figure 15 shows typical phase orbit plots for a buncher capable of accepting all of the injected current irrespective of phase relationship.

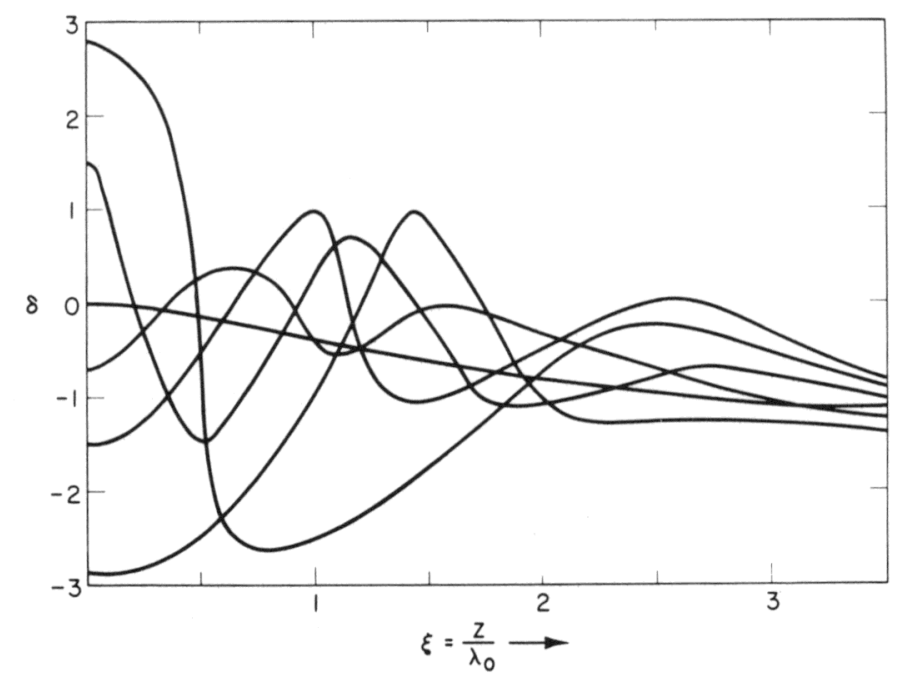

Fig. 15. Electron Phase Oscillations Within a Non-uniform, High Capture Buncher Section 
Disadvantages of this type of buncher include relatively wide energy spread of the emergent electron beam and reduced energy gain owing to the oscillatory nature of the phase orbits.

A further disadvantage of this type of high capture buncher is that owing to the diverse nature of the phase orbits a unique value of axial magnetic field distribution along the length of the buncher cannot control the various radial dispersion forces associated with all of the orbits.

A buncher which is designed to prevent phase oscillations will usually have a reduced acceptance associated with it. This loss in current, however, may be readily recovered by the use of a prebuncher. A buncher can also be arranged such that the energy spread of the accelerated electrons, due to velocity modulation and phase variations at injection, can be reduced to approximately $50 \mathrm{kev}$ in several $\mathrm{Mev}$.

Elimination of phase oscillations results in a higher overall gain in energy from a given length of buncher and also enables a graded axial magnetic field to be applied which more readily matches the focus requirements of all the accepted electrons.

By combining the above features with a parallel (or slightly divergent) injected beam, a high-energy electron beam of small cross section can be obtained at the end of a long accelerator section. Figure 16 shows the impression of an electron beam on a film placed against a thin metal window at the end of a $250-\mathrm{cm}$ section of accelerator guide which was operated with solenoidal focusing over only the first $60 \mathrm{~cm}$ of length.

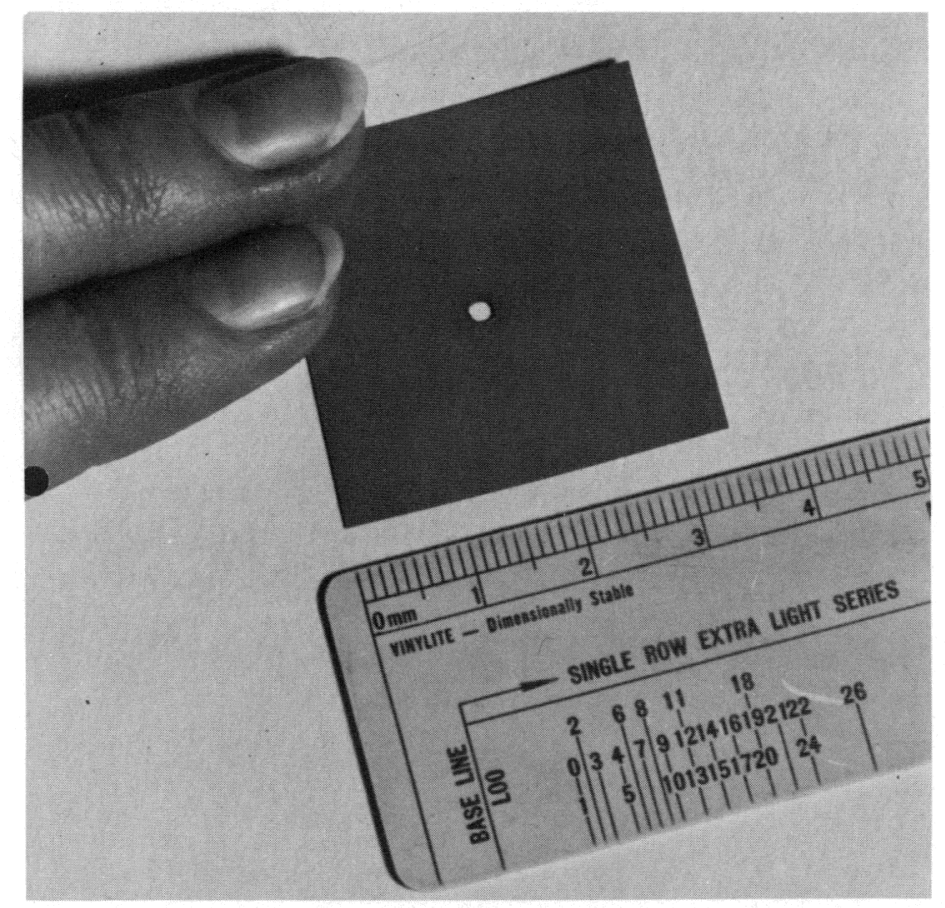

Fig. 16

Impression of Straight Ahead Electron Beam on a Film Which Was Placed Against a Thin Metal Window at the End of the Accelerator Tube 


\section{References}

1. D. W. Fry, R. B. R. S. Harvie, L. B. Mullett, and W. Walkinsaw, Travelling-wave Linear Accelerator for Electrons, Nature 160, 351-353 (Sept 13, 1947).

2. E. L. Chu and W. W. Hansen, The Theory of Disk-loaded Wave Guides, J. App. Phys., 18, 996-1008 (Nov 1947).

3. J. C. Slater, The Design of Linear Accelerators, Revs. Mod. Phys., 20, 473-518 (July 1948).

4. L. B. Mullett and B. G. Loach, Experimental Work on Corrugated Wave-guides and Associated Components for Linear Electron Accelerators, Proc. Phys. Soc. (London), 61, 271-284 (1948).

5. M. Chodorow, E. L. Ginzton, W. W. Hansen, R. L. Kyhl, R. B. Neal, and W. K. H. Panofsky, Stanford High Energy Linear Electron Accelerator (Mark III), Rev. Sci. Inst., 26, 134-204 (Feb 1955).

6. J. H. Cusick and J. Haimson, $10 \mathrm{Mev}$ Rotating Target Linear Accelerator for Radiography of Large Rocket Motors, Missiles and Rockets Symposium, U. S. Naval Ammunition Depot, Concord, California, April 1961.

7. J.. Haimson, Radiography of Large Missiles with the Linear Electron Accelerator, presented at WESCON, San Francisco, August 1961.

8. A. E. Harrison, Klystron Tubes, McGraw-Hill Book Co., Inc., New York (1947).

\section{Discussion}

SOME DESIGN ASPECTS OF LINEAR ACCELERATORS USED FOR HIGH ENERGY X-RAY PRODUCTION

R. Goldman, General Electric Company: This rotating head looks as though it would be a big advantage in radiographic inspection. On the other hand, this linear accelerator is a big sort of vacuum tube, and if you have a rotating head you must have a rotating vacuum seal. The rotating head ought to be a big advantage, but a continuous pumping system is a problem. What do you do about pumping these things?

J. Haimson: I should have brought this point out. The linacs use an electronic pumping system - a Vac-Ion pump. These are highly advanced methods of producing extremely high vacuums; there are no liquid components. It is a tantalum-bombardment process, and with such equipment 
you can keep your systems down to $10^{-9} \mathrm{~mm}$. This means that all metal vacuum seals can be used without the use of neoprenes or any other of the rubber O-ring insertions. The system can be baked out, which gives extremely contaminant-free surfaces and produces very high vacuums. Back in 1951, an 8-Mev linac was used for medical therapy; it essentially was a 3-m-long machine. It had a rotating head, and that machine is still in action, treating something like 40 patients a day over the last 8 years. They change the seals once every 6 mo because of radiation damage. Now in our equipment, which is the more modern approach, we have used cascade seals which are buried in protective absorbing material so that the radiation cannot reach them. These seals are also surrounded by a water-cooling jacket.

\section{R. Goldman: Are they all metal seals?}

J. Haimson: No, these are graphite seals which are quite crude and quite satisfactory.

R. Goldman: What is the pumping speed of your pump?

J. Haimson: Pumping speed is something of the order of 75 liters/sec.

R. Goldman: How do you start this thing up?

J. Haimson: The usual mechanical pump is used to rough the system down to a micron, and the Vac-Ion pump is hit with about $6 \mathrm{kv}$, and it takes over from there. You can then switch the mechanical pump off. The VacIon pump concept is quite a big advance in this.

R. Goldman: It can, then, run with this ion pump, say 6 months, with no additional pumping of any kind except what the ion pumps do?

J. Haimson: That is right - just the ion pump alone. There is also the advantage of maneuverable machines, because if you have a Vac-Ion pump you're not interested in having any fore-pump connection, or any fore-pump seals. The Vac-Ion pump is located right on the tube itself, which is what we do, and, therefore, the whole machine is maneuverable. There is no connection through any form of mechanical, or other lowpressure, seal, and there is no necessity to run a mechanical pump to back up the Vac-Ion pump as is the case with diffusion pumps.

R. Peterson, Argonne National Laboratory: What was the length of your accelerating region?

J. Haimson: You could design these machines in a variety of ways. The particular machine shown in Fig. 7 has $2 \frac{1}{2} \mathrm{~m}$ of accelerating structure. There is an additional $15 \mathrm{in.}$ of bending X-ray head, and some $24 \mathrm{in}$. at the other end to take care of the drift space section and the electron gun itself. The overall machine including the fabrication and the housing is $13 \mathrm{ft} 3 \mathrm{in}$. long, but they can be made shorter or longer. 
TOUR PHOTOGRAPHS OF THE ZGS FACILITIES
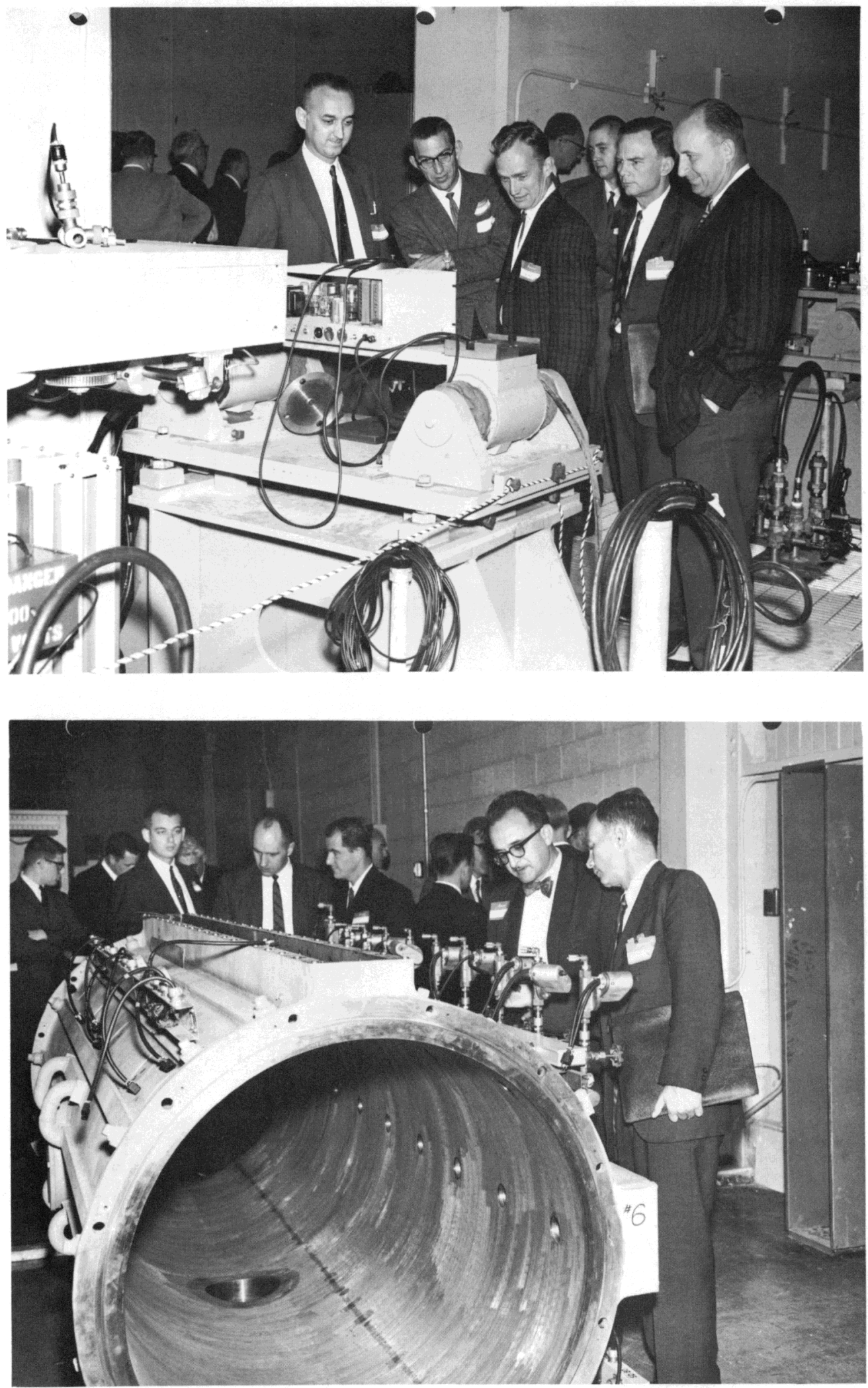


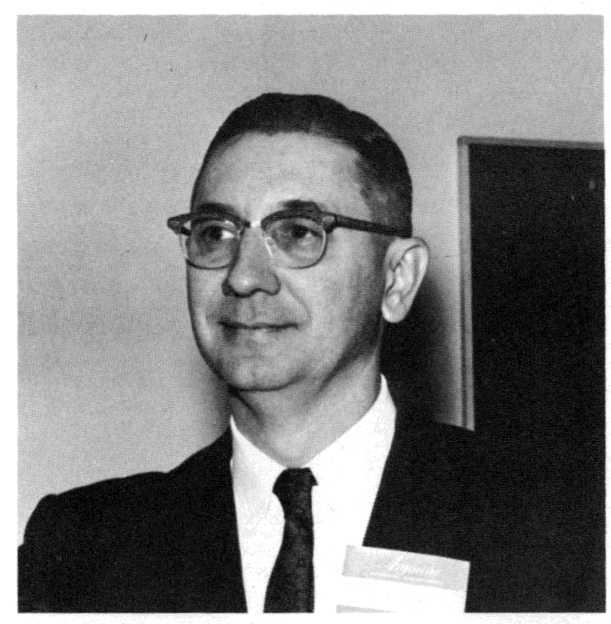

Mr. Albert H. Allard

Albert H. Allard joined the Eastman

Kodak Company, Department of Photographic Chemistry, Kodak Research Laboratories, in 1948 following graduation from Cornell University with a BS degree in Biochemistry. Later he was transferred to the X-ray Division as a technical representative, covering various areas in the Midwest and East. In 1957, Mr. Allard returned to Rochester as a product specialist, organized and developed the curriculum, and directed the X-Omat Training Center. He also supervised X-

Omat training courses for European personnel in London, and later for Latin American personnel in Mexico City. In 1959, he became coordinator of educational and publication activities of the X-ray Sales Division. He is now Supervisor, X-ray Technical Services, Eastman Kodak Company.
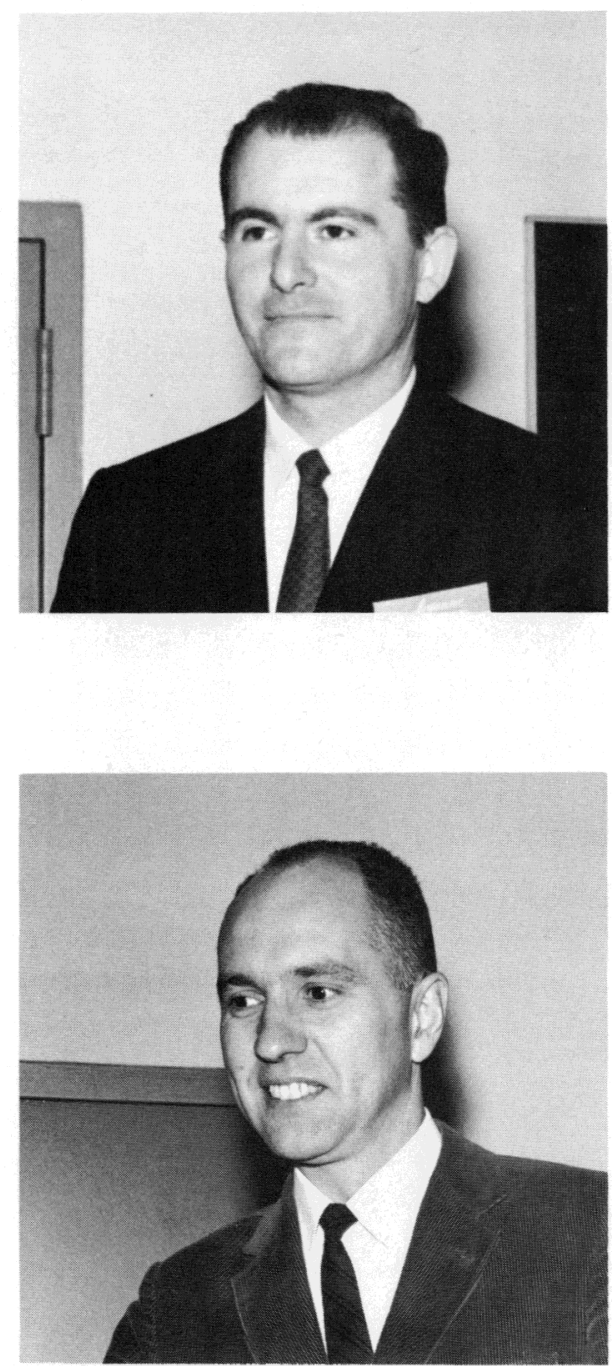

Mr. Harold Berger

Harold Berger is an Associate Physicist with the Metallurgy Division of Argonne National Laboratory. He received his BS and MS degrees in Physics from Syracuse University in 1949 and 1951, respectively. Before joining Argonne National Laboratory, Mr. Berger was associated with Battelle Memorial Institute and the General Electric Company. He is a member of the American Physical Society, The Society for Nondestructive Testing, and the Transducer Compendium Committee of the Instrument Society of America.

\section{Mr. Norman S. Beyer}

Norman S. Beyer has been as Associate Physicist with the Special Materials and Services Division of Argonne National Laboratory since 1957. He holds a BS in Mechanical Engineering (Aero Specialty) and $\mathrm{MA}$ in Physics. His publications are in the fields of radiological physics, mass spectroscopy, and nondestructive testing. Previous to joining the Argonne staff, Mr. Beyer was active in biophysics, radiation dosimetry, university teaching, and nuclear engineering.

Currently, he is engaged in research and development work relative to nondestructive testing by radiation-detection methods. 

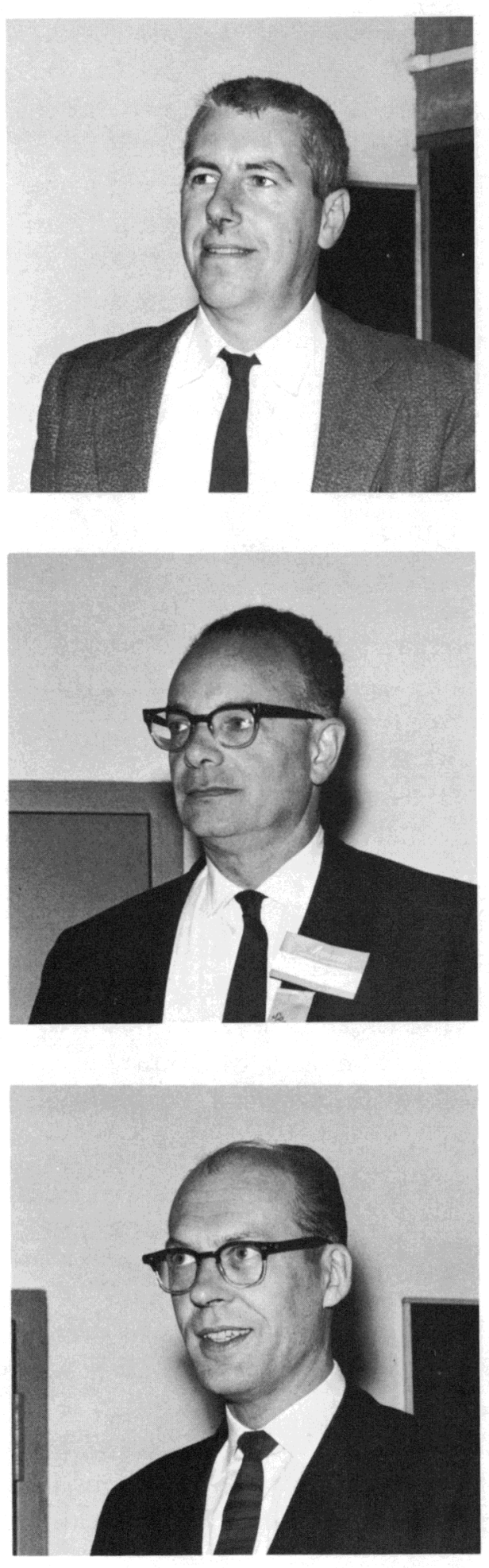

Dr. Thomas H. Blewitt

Thomas H. Blewitt is the Irradiation Effects Group Leader at Argonne National Laboratory. He received his BS and DSc in Physics from Carnegie Institute of Technology. Before coming to Argonne in 1961 he spent 10 years as a Group Leader in the Solid State Division of Oak Ridge National Laboratory.
Hans E. Bömmel is a citizen of Switzerland and obtained his degree in Phys ics from the University of Zurich in 1943. He was Professor of Ultrasonics and Nuclear Physics at the University of Zurich until 1953, when he joined the Bell Telephone Laboratories. At present, he is on leave from Bell Telephone Laboratories to the University of California as Professor of Physics.

Mr. E. Alfred Burrill

E. Alfred Burrill is Vice President and Director of Marketing at the High Voltage Engineering Corporation, Burlington, Mass. He received his BS in Physics from the Massachusetts Institute of Technology in 1943. Since graduation he has held several positions with the High Voltage Engineering Corporation. He is a member of the following societies: American Physical Society, American Nuclear Society, American Society for Testing Materials, Society for Nondestructive Testing, Atomic Industrial Forum, and the American Association for the Advancement of Science. 

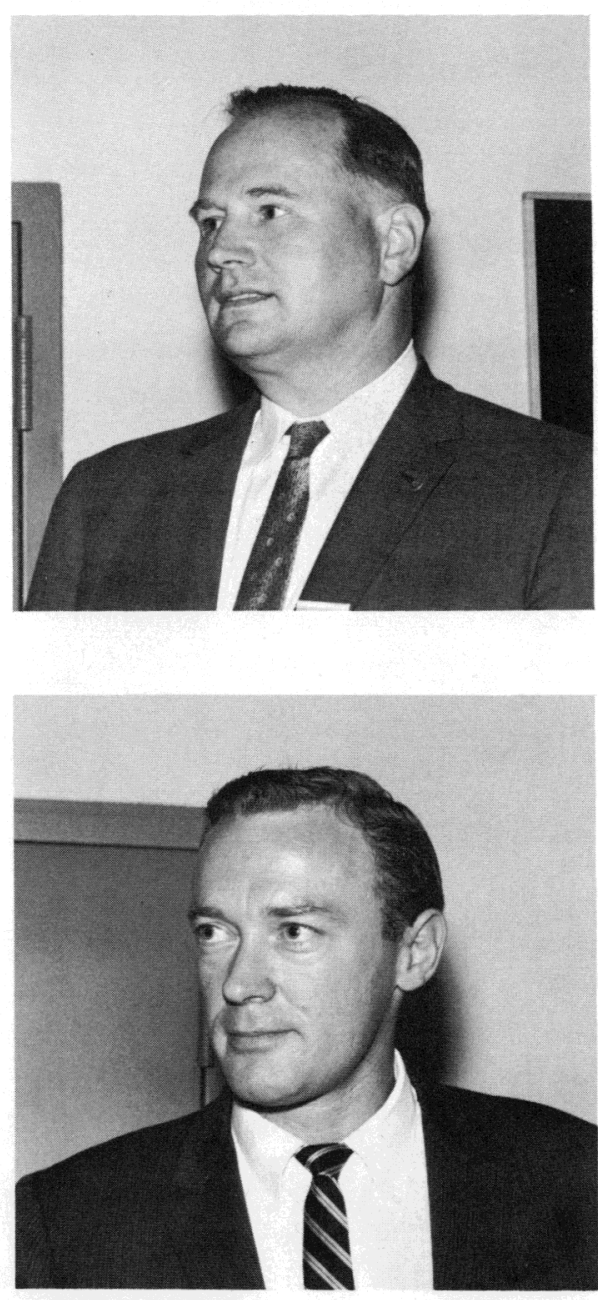

Mr. Robert S. Goodyear

Robert S. Goodyear is President and General Manager of Fenwal Electronics, Inc., Framingham, Mass. He received his BS degree from Harvard University with honors in Physics in 1936. Since 1936 he has served at U.S. Rubber, Western Electric, North American Research Laboratories, Inc., and Victory Engineering. Since his graduation he has been concerned with thermistors and developing of instrumentation for use with thermistors.

Mr. Jacob Haimson

Jacob Haimson, Manager, Accelerator Research at Varian Associates, Palo Alto, California, is an engineer as well as a physicist, and has previously been associated with the General Electric Company and Metropolitan Vickers in England as a design engineer-physicist. He has been engaged in linear accelerator work since 1953 and was appointed superintending engineer for linac projects in Europe, Australia, and New Zealand before joining Varian as Project Physicist for linear accelerator development in 1959. He holds a degree in Engineering from the Melbourne Institute of Technology and a degree in Science with honors in Physics from the University of Melbourne. He is a recognized expert in accelerator design and a consultant in radiotherapy applications.

Mr. Jule Hirschfield

Jule Hirschfield is Project Engineer in the Applied Physics Department at High Voltage Engineering Corporation. He received his BS degree in Electrical Engineering from the Carnegie Institute of Technology. Before joining High Voltage Engineering Corporation in 1959 he was Unit Chief in the X-Ray Division of the Naval Ordnance Laboratory and held a number of positions in private industry engaged in nondestructive testing developments and applications. 

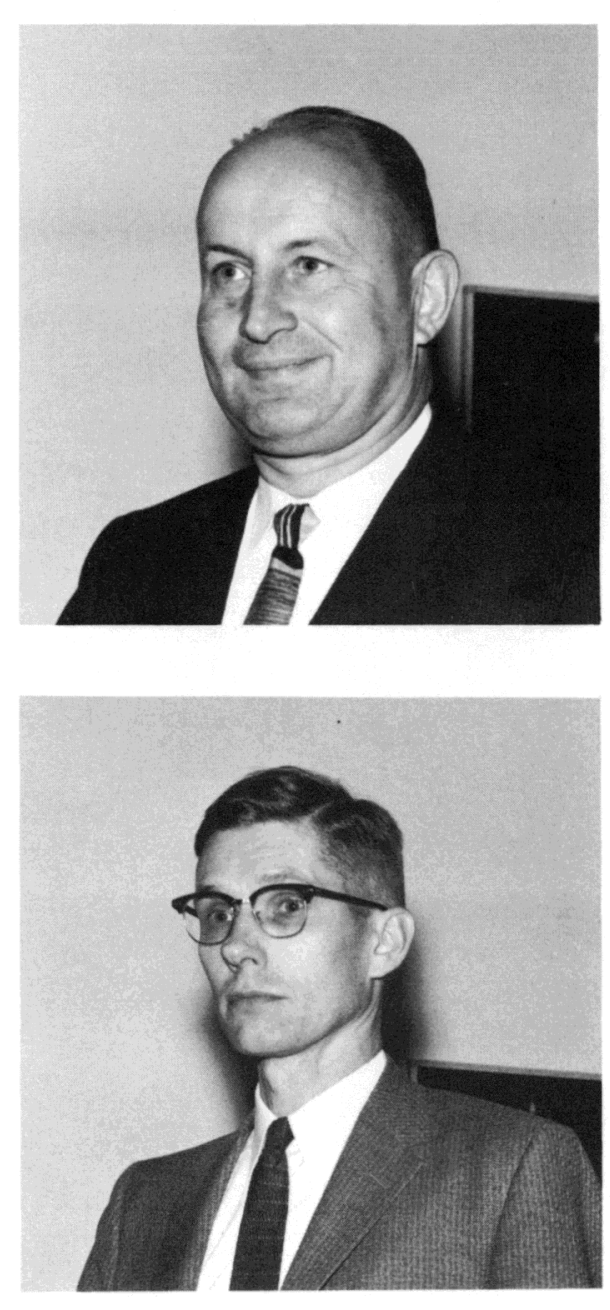

Mr. William D. Kiehle

William D. Kiehle is Manager of Industrial X-Ray Sales, Eastman Kodak Company, Rochester, N. Y. He received a degree in Administrative-Mechanical Engineering from Cornell University. Since graduation he has been employed by Eastman Kodak, except for time out during World War II. At Eastman Kodak he has specialized in the field of industrial radiography.

Mr. Ernest T. Larson

Ernest T. Larson is a Section Leader of the X-ray Research and Development Group at Ansco. He received his BS degree in Physics from the University of Pittsburgh and his M.S. degree from Massachusetts Institute of Technology. In 1945 he joined General Aniline and Film Corporation. He has served in the Central Research Laboratories and in the Ansco Division of the Corporation. He has published several papers on fundamental work concerned with latent image formation. He also holds a number of patents in this field.

Dr. Robert J. Maurer

Robert J. Maurer is Professor of Physics at the University of Illinois. He received his BS in Chemistry and his PhD in Physics from the University of Rochester. He has taught at M.I.T., University of Pennsylvania, and Carnegie Institute of Technology. He also served as a Physicist on the Manhattan Project at the University of Chicago, and for a year was head of the Physics Branch of the Office of Naval Research.

Dr. Heinz F. Nitka

Heinz F. Nitka is Associate Director for Research and Development at the Ansco Division of General Aniline and Film Corporation. He received his $\mathrm{PhD}$ in Physics from the University of Heidelberg, Germany. He has been in his present position since 1954. His previous experience includes research and development at The German National Bureau of Standards, Agfa Film Plant, and Imperial Chemical Industries, Ltd. 


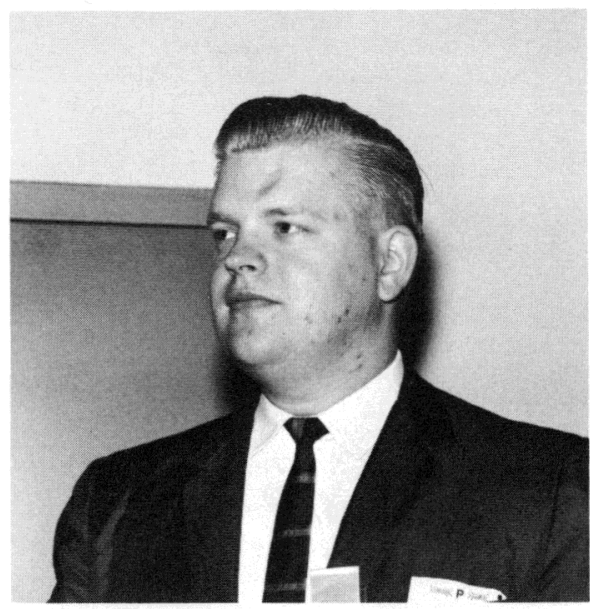

ultrasonic attenuation in solids. He is a member of the American Physical Society.
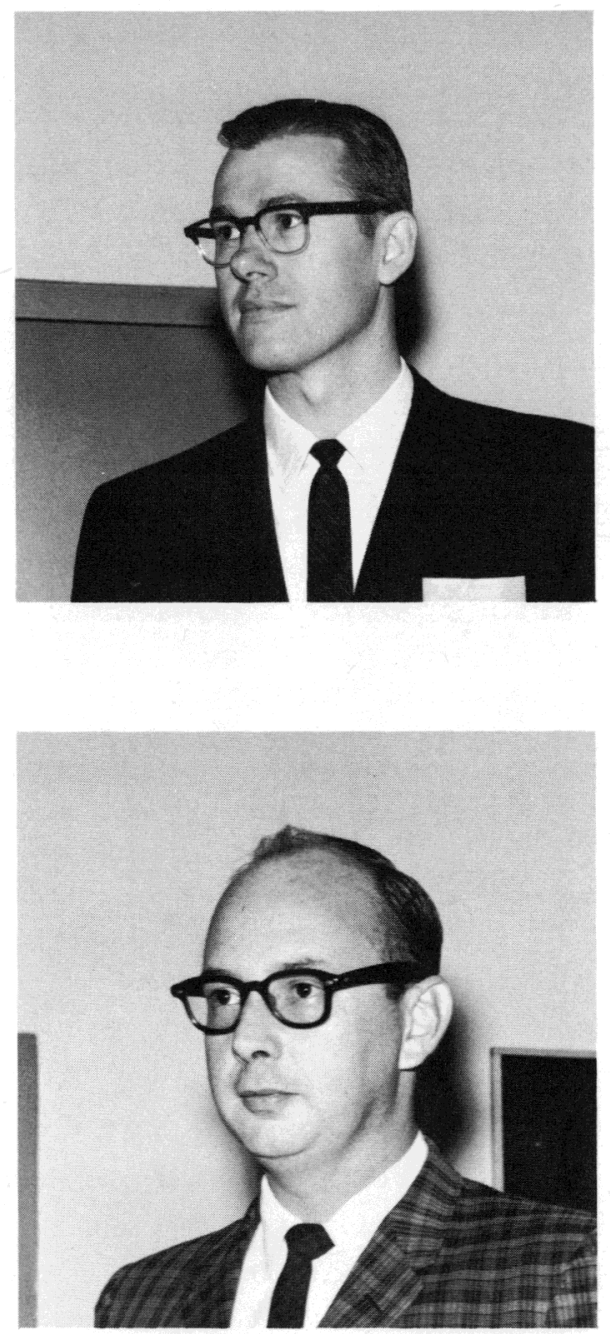

Mr. Ronald G. Peterson

Ronald G. Peterson is an Assistant Physicist with the Metallurgy Division of Argonne National Laboratory. He was graduated from the University of Illinois with a BS in Physics in 1954 and MS in Physics in 1956. Previously, he was associated with the Hoffman Semiconductor Division of the Hoffman Electronics Corporation and with the Joslyn Manufacturing and Supply Company. Since he became associated with Argonne National Laboratory in 1959, his work has been concerned with the measurement of Mr. Fred R. Rollins, Jr.

Fred R. Rollins received the MS degree in Physics from the University of Kansas in 1952. He was an officer in the U.S. Navy for $3 \frac{1}{2}$ yr prior to joining Midwest Research Institute, Kansas City, in 1955. At Midwest Research Institute, Mr. Rollins has worked primarily in the field of electron microscopy and ultrasonics. For the past several years he has been studying the propagation of high-frequency shear waves in various solids and the use of such waves in the detection and measurement of internal stresses.

Mr. Richard R. Rowand

Richard R. Rowand is Acting Chief of Applied Mechanics Section, ASDA, Wright-Patterson Aeronautical Systems Division, Wright-Patterson Air Force Base, Ohio. He has been with Wright-Patterson since 1951 and since 1955 has been concerned primarily with the development of nondes tructive testing. $\mathrm{Mr}$. Rowand received his BA degree from Ohio State University. He has published a number of papers in the field of nondestructive testing and is a member of a number of technical societies. 


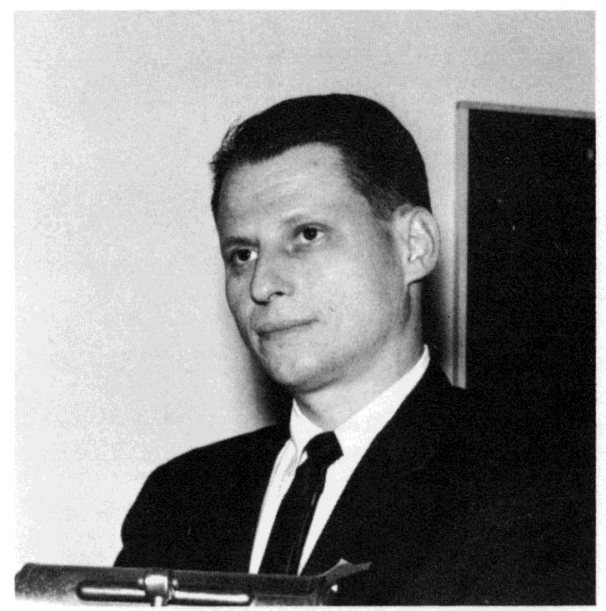

Mr. Harold L. Sachs

Harold L. Sachs is Infrared Instrumentation Group Leader with the PerkinElmer Corporation, Norwalk, Connecticut, and is responsible for their current ARGMA supported program in the application of these techniques to the nondestructive tests of solid propellent missile cases. He received his BS in EE at the University of Pennsylvania in 1951, and performed graduate studies at Brooklyn Polytechnic Institute. Mr. Sachs is a member of Theta Kappa $\mathrm{Nu}$ and other professional groups. Before joining Perkin-Elmer in December 1959, he was with the Production Research Corporation, Barnes Engineering Company and Servo Corporation of America.

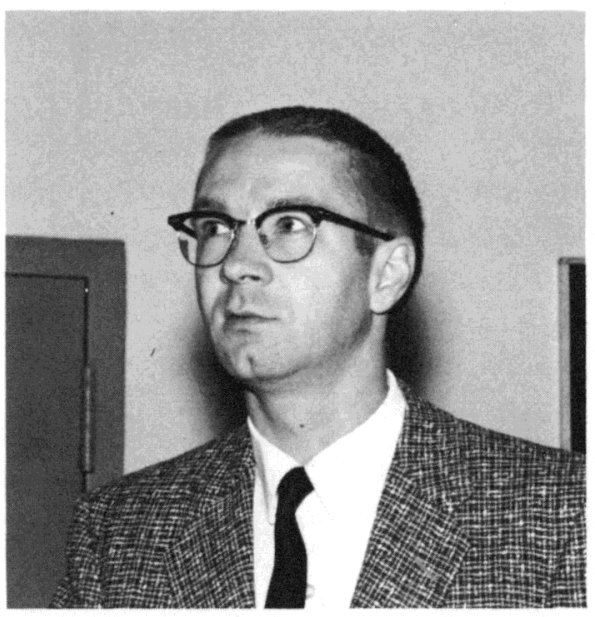

Mr. Harold V. Watts

Harold V. Watts is a Research Physicist with the Physics Division of Armour Research Foundation. He received his BS and MS degrees in Physics from the Illinois Institute of Technology in 1950 and 1953, respectively, and is currently completing work for his $\mathrm{PhD}$ at the same institution. He has been with the Armour Research Foundation since 1950, during which time he has worked on various projects concerning the application of radioisotopes and nuclear techniques for nondestructive inspection and testing. Mr. Watts has several publications and has presented technical talks on this subject. He is a member of the American Physical Society, the American Nuclear Society, and Sigma Xi. 
ATTENDEES

Kenneth Allan

Hercules Powder Company

La Vale, Maryland

Albert H. Allard

Eastman Kodak Company

Rochester, New York

Bruce Anderson, Jr.

U.S. Atomic Energy Commission

Argonne, Illinois

C. Arne Arenberg

Metallurgy Division

Argonne National Laboratory

Argonne, Illinois

Edna M. Bahr

Metallurgy Division

Argonne National Laboratory

Argonne, Illinois

K. Balaramamoorthy

Atomic Energy Establishment

Bombay, India

A. G. Barkow

Marquette University

Milwaukee 3, Wisconsin

H. Berger

Metallurgy Division

Argonne National Laboratory

Argonne, Illinois

Alan Berndt

Metallurgy Division

Argonne National Laboratory

Argonne, Illinois

Norman S. Beyer

Special Materials and Services

Division

Argonne National Laboratory

Argonne, Illinois
J. E. Bigelow

General Electric Company

Milwaukee, Wisconsin

T.H. Blewitt

Metallurgy Division

Argonne National Laboratory

Argonne, Illinois

$\mathrm{H}$. V.Bohm

Wayne State University

Detroit, Michigan

H. E. Bo̊mmel

University of California

Los Angeles, California

Louis M. Bonnefond

U.S. Atomic Energy Commission

Washington, D. C.

Henry J. Boulanger

Texas Instruments Incorporated

Attleboro, Massachusetts

Bruce L. Bramfitt

Missouri School of Mines

Rolla, Missouri

E. Alfred Burrill

High Voltage Engineering

Corporation

Burlington, Massachusetts

John W. Campbell

Street and Smith Publishing

Company

New York City, New York

Richard Carlander

Metallurgy Division

Argonne National Laboratory

Argonne, Illinois 
Andrew J. Cassell

International Institute of

Nuclear Science and Engineering

Argonne National Laboratory

Argonne, Illinois

Roshan L. Chaddha

Kansas State University

Manhattan, Kansas

Keith R.Clark

High Voltage Engineering

Corporation

La Grange, Illinois

John W. Colby

National Lead Company of Ohio

Cincinnati, Ohio

Raymond E. Conchieri

Springfield Armory

Springfield, Massachusetts

K. V. Cook

Oak Ridge National Laboratory

Oak Ridge, Tennessee

George M. Corney

Eastman Kodak Company

Rochester, New York

George Costello

University of Illinois

Champaign, Illinois

A. V. Crewe

Laboratory Director's Office

Argonne National Laboratory

Argonne, Illinois

P.E. Dempsey

U.S. Steel Corporation

Gary, Indiana
Robert E. Dickens

Metallurgy Division

Argonne National Laboratory

Argonne, Illinois

Roberta Ann di Novi

Metallurgy Division

Argonne National Laboratory

Argonne, Illinois

William B. Doe

Remote Control Engineering

Argonne National Laboratory

Argonne, Illinois

W. Lyle Donaldson

Southwest Research Institute

San Antonio, Texas

R. Dean Dragsdorf

Kansas State University

Manhattan, Kansas

James W. Dutli

Los Alamos Scientific Laboratory

Los Alamos, New Mexico

D. L. Dye

Boeing Company

Seattle, Washington

Paul D. Edwards

Los Alamos Scientific Laboratory

Los Alamos, New Mexico

D. N. Eggenberger

Electronics Division

Argonne National Laboratory

Argonne, Illinois

G. R. Ellis on

Sandia Corporation

Albuquerque, New Mexico 
Louis Ellsworth

Kansas State University

Manhattan, Kansas

D. S. Eppelsheimer

Missouri School of Mines

Rolla, Missouri

G. B. Evridge

Eastman Kodak Company

Rochester, New York

K. E. Feith

Armour Research Foundation

Chicago, Illinois

Edward S. Fisher

Metallurgy Division

Argonne National Laboratory

Argonne, Illinois

J. D. Fisler

Eastman Kodak Company

Chicago, Illinois

R. D. Flatland

Industrial X-Ray Engineers

Franklin Park, Illinois

James R. Gates

Service Pipe Line Company

Joliet, Illinois

Bernard F. Geren

Special Materials and Services

Division

Argonne National Laboratory

Argonne, Illinois

Augusto Gerosa

Comitato Nazionale L'Energia

Nucleare

Ispra (Varese), Italy

J. D. Glomb

Continental Can Company

Chicago, Illinois
R. Goldman

General Electric Company

Schenectady, New York

Leonard Goodman

Atomics International

Canoga Park, California

Robert S. Goodyear

Fenwal Electronics, Incorporated

Framingham, Massachusetts

D.R. Green

General Electric - Hanford

Atomic Products Operation

Richland, Washington

D. E. Grosvenor

Chemical Engineering Division Argonne National Laboratory

Argonne, Illinois

L. B. Gulbransen

Washington University

St. Louis, Missouri

Sally Gustafson

Metallurgy Division

Argonne National Laboratory

Argonne, Illinois

J. Haims on

Varian Associates

Palo Alto, California

Guest Hake

Atomic Energy of Canada

Toronto, Ontario, Canada

R. S. Halladay

The Budd Company

Oak Park, Illinois

Raymond K. Hart

Metallurgy Division

Argonne National Laboratory

Argonne, Illinois 
E. Dane Harvey

Hercules Powder Company

Cumberland, Maryland

Herbert D. Hendricks

Savannah River Laboratory

Aiken, South Carolina

James W. Henning

Graphic Arts Department

Argonne National Laboratory

Argonne, Illinois

Allan G.Hins

Metallurgy Division

Argonne National Laboratory

Argonne, Illinois

Jule Hirschfield

High Voltage Engineering Corporation

Brookline, Massachusetts

James A. Holloway

Naval Ordnance Laboratory

White Oak, Maryland

James A. Horak

Metallurgy Division

Argonne National Laboratory

Argonne, Illinois

B. A. Howard, Jr.

Rock Island Arsenal

Rock Island, Illinois

John Howell

General Electric Company

Milwaukee, Wisconsin

William L. Hughes

Boeing Company

Seattle, Washington

Louis Ianniello

Metallurgy Division

Argonne National Laboratory

Argonne, Illinois
Robert L. Irwin

Metallurgy Division

Argonne National Laboratory

Argonne, Illinois

Richard O. Ivins

Chemical Engineering Division

Argonne National Laboratory

Argonne, Illinois

William J. Jarvis

Dunlee Corporation

Bellwood, Illinois

Donald E. Jones

Kansas State University

Manhattan, Kansas

T. W. Judd

Republic Steel Corporation

Cleveland, Ohio

C. Nelson Julian

Springfield Armory

Springfield, Massachusetts

S. Kaufmann

Reactor Engineering Division

Argonne National Laboratory

Argonne, Illinois

Harry B. Kern

The Martin Company

Baltimore, Maryland

Robert Kessie

Chemical Engineering Division

Argonne National Laboratory

Argonne, Illinois

William D. Kiehle

Eastman Kodak Company

Rochester, New York

W. R. Kimel

Kansas State University

Manhattan, Kansas 
K. K. Klindt

Union Carbide Nuclear Corporation

Oak Ridge, Tennessee

Verne M. Kolba

Reactor Engineering Division Argonne National Laboratory

Argonne, Illinois

Anthony R. Konecny

Washington University

Berkeley, Missouri

Karl A. Krasim

Chicago Bridge \& Iron Company

Chicago, Illinois

Irvin R. Kraska

Metallurgy Division

Argonne National Laboratory

Argonne, Illinois

A.B. Krisciunas

Laboratory Director's Office

Argonne National Laboratory

Argonne, Illinois

T. F。 Krohne

Laboratory Director's Office

Argonne National Laboratory

Argonne, Illinois

Gordon P. Krueger

University of Wisconsin

Madison, Wisconsin

E. T. Larson

Ansco Corporation

Binghamton, New York

W. E. Lawrie

Armour Research Foundation

Chicago, Illinois

William Z. Leavitt

Watertown Arsenal

Watertown, Massachusetts
Sheldon Leonard

Lockheed Aircraft Corporation

Sunnyvale, California

Robert C. Liimatainen

Chemical Engineering Division

Argonne National Laboratory

Argonne, Illinois

K. Lucas

Allegany Ballistics Laboratory

Cumberland, Maryland

A. D. Lucian

Aerojet General Corporation

Sacramento, California

Robert F. McDevitt

Gary Steel Works

Gary, Indiana

W. J. McGonnagle

Metallurgy Division

Argonne National Laboratory

Argonne, Illinois

William N. McNerney

The Martin Company

Baltimore, Maryland

D. E. MacLeod

Metallurgy Division

Argonne National Laboratory

Argonne, Illinois

Paul B. Maertz

A. O. Smith Corporation

Milwaukee, Wisconsin

Dale R. Maley

Automation Industries, Incorporated

Boulder, Colorado

H. M. Mann

Electronics Division

Argonne National Laboratory

Argonne, Illinois 
Carrell R. Marshall

Taylor Forge \& Pipe Works

Cicero, Illinois

Harley E. May

General Electric - Hanford

Atomic Products Operation

Richland, Washington

K. L. Merkle

Metallurgy Division

Argonne National Laboratory

Argonne, Illinois

S. Z. Mikhail

Kansas State University

Manhattan, Kansas

Donald P. Moak

Battelle Memorial Institute

Columbus, Ohio

John Morgan

Metallurgy Division

Argonne National Laboratory

Argonne, Illinois

Richard B. Moyer

The Carpenter Steel Company

Reading, Pennsylvania

Richard Muenow

Portland Cement Association

Skokie, Illinois

Lawrence A. Neimark

Metallurgy Division

Argonne National Laboratory

Argonne, Illinois

W. G. Nilson

Ebasco Services, Incorporated

New York, New York

Mary Jane Oestmann

International Institute

Argonne National Laboratory

Argonne, Illinois
John W. Orner

Watertown Arsenal

Watertown, Massachusetts

B. Ostrofsky

American Oil Company

Whiting, Indiana

Milton Peach

Michigan College of Mining

and Technology

Houghton, Michigan

Ronald B.Perry

Metallurgy Division

Argonne National Laboratory

Argonne, Illinois

Ronald G. Peterson

Metallurgy Division

Argonne National Laboratory

Argonne, Illinois

Levan M. Phelps

Boeing Company

Seattle, Washington

Fred T. Phelps

Solid State Science Division

Argonne National Laboratory

Argonne, Illinois

John H. Pingel

Industrial Hygiene and Safety

Division

Argonne National Laboratory

Argonne, Illinois

William R. Plant

Knolls Atomic Power Laboratory

Schenectady, New York

W. Podhradsky

Metallurgy Division

Argonne National Laboratory

Argonne, Illinois 
Roy G. Post

University of Arizona

Tucson, Arizona

Edward E. Potter

Commonwealth Edison Company

Maywood, Illinois

Robert H. Rakel

University of Cincinnati

Cincinnati, Ohio

Kenneth W.Rarey

Continental Can Company,

Inc or por ated

Chicago, Illinois

D. W. Rathmann

Thiokol Chemical Corporation

Tremonton, Utah

T. Reim

Republic Steel Corporation

Cleveland, Ohio

C.J.Renken

Metallurgy Division

Argonne National Laboratory

Argonne, Illinois

Mordy M. Rhodes

Branson Instruments, Incorporated

Chicago, Illinois

John H. Roberson

Associated Midwest Universities

Argonne, Illinois

L. E. Robinson

Reactor Engineering Division

Argonne National Laboratory

Argonne, Illinois

M. Rodin

Reactor Engineering Division

Argonne National Laboratory

Argonne, Illinois
Fred R. Rollins, Jr.

Midwest Research Institute

Kansas City, Missouri

Moshe Rosen

Metallurgy Division

Argonne National Laboratory

Argonne, Illinois

H. V.Ross

Management Systems

Argonne National Laboratory

Argonne, Illinois

Richard R. Rowand

Wright-Patterson Air Force Base

Wright-Patterson, Ohio

E. W. Rylander

Laboratory Director's Office

Argonne National Laboratory

Argonne, Illinois

Harold L. Sachs

Perkin-Elmer Corporation

Norwalk, Connecticut

Allan Sather

Metallurgy Division

Argonne National Laboratory

Argonne, Illinois

Charles W. Sayles

Reactor Engineering Division

Argonne National Laboratory

Argonne, Illinois

Donald R. Schaffer

A. O. Smith Corporation

Milwaukee, Wisconsin

Robert D. Schamberger

United Nuclear Corporation

Pawling, New York

John Schieb

Picker X-Ray Company

Chicago, Illinois 
D. R. Schmitt

Metallurgy Division

Argonne National Laboratory

Argonne, Illinois

J. W. Seifert

Babcock \& Wilcox Company

Alliance, Ohio

Ronald H. Selner

Metallurgy Division

Argonne National Laboratory

Argonne, Illinois

Steven Serabian

Avco Corporation

Wilmington, Massachusetts

Michael W. Sewew

Pennies Industrial X-Ray Company

Chicago, Illinois

Guy H. B. Shaffer

U.S. Naval Submarine Base

New London, Connecticut

Alexander J. Sielicki

A. O. Smith Corporation

Milwaukee, Wisconsin

Neil Sinclair

General Dynamics Corporation

Gales Ferry, Connecticut

Thomas Slattery

Fairchild Publications

Chicago, Illinois

Karl F. Smith

Metallurgy Division

Argonne National Laboratory

Argonne, Illinois

Sherman D. Smith

Metallurgy Division

Argonne National Laboratory

Argonne, Illinois
P. D. Southgate

Armour Research Foundation

Chicago, Illinois

Paul F. Stablein

Metallurgy Division

Argonne National Laboratory

Argonne, Illinois

David Stein

Picatinny Arsenal

Dover, New Jersey

R. F. Swanson

New York Central Railroad -

Technical Research

Cleveland, Ohio

Chester T. Szymko

Central Shops

Argonne National Laboratory

Argonne, Illinois

E. C. Thomas

Great Lakes Carbon Cor poration

Niagara Falls, New York

Marvin M. Turkanis

Nuclear Materials \& Equipment

Corporation

Apollo, Pennsylvania

Louis A. Turner

Laboratory Director's Office

Argonne National Laboratory

Argonne, Illinois

R. L. Uphoff

Branson Instruments

Stamford, Connecticut

Donald Van Ostenburg

Metallurgy Division

Argonne National Laboratory

Argonne, Illinois 
Rex E.Vincent

Chicago Bridge \& Iron Company

Chicago, Illinois

F. H. Vitovec

University of Wisconsin

Madison, Wisconsin

Milton Volpe

Metallurgy Division

Argonne National Laboratory

Argonne, Illinois

Bill Walters

Tuboscope Company

Houston, Texas

Harold V. Watts

Armour Research Foundation

Chicago, Illinois

D. G. Westlake

Metallurgy Division

Argonne National Laboratory

Argonne, Illinois

Bruce E. Webster

Graphic Arts Department

Argonne National Laboratory

Argonne, Illinois

Helmut Weik

University of Cincinnati

Cincinnati, Ohio

Fred K. White

U.S. Atomic Energy Commission

Argonne, Illinois

John K. White

Jones \& Laughlin Steel

Pittsburgh, Pennsylvania

Ross Whitehead

Steel Magazine

Cleveland, Ohio
W.J.Whitfield

Sandia Corporation

Albuquerque, New Mexico

Everett E. Wicker

U.S. Steel Corporation

Monr oeville, Pennsylvania

C. M. Wiley

Electronics Magazine

Chicago, Illinois

Ernest Wilms

University of Illinois

Urbana, Illinois

Robert L. Wright

Missouri School of Mines

Rolla, Missouri

Eugene A. Zemba

Special Materials and Services

Division

Argonne National Laboratory

Argonne, Illinois

Milt Zeutschel

General Electric - Hanford

Atomic Products Operation

Richland, Washington 